\title{
Growth and Wetting Properties of \\ Carbon Nanofibers
}




\section{GROWTH AND WETTING PROPERTIES \\ OF}

\section{CARBON NANOFIBERS}

Hrudya Nair 


\section{Graduation Committee}

\begin{tabular}{|c|c|}
\hline Prof. Dr. Ir. Hans Hilgenkamp (Secretary) & University of Twente, The Netherlands \\
\hline Prof. Dr. Ir. Leon Lefferts (Promoter) & University of Twente, The Netherlands \\
\hline Prof. Dr. Detlef Lohse (Promoter) & University of Twente, The Netherlands \\
\hline Dr. Arie van Houselt (Assistant Promoter) & University of Twente, The Netherlands \\
\hline Prof. Dr. Daniel Bonn & University of Amsterdam, The Netherlands \\
\hline Prof. Dr. Harry Bitter & University of Wageningen, The Netherlands \\
\hline Prof. Dr. Han Gardeniers & University of Twente, The Netherlands \\
\hline Dr. Chao Sun & University of Twente, The Netherlands \\
\hline Dr. Roald M. Tiggelaar & University of Twente, The Netherlands \\
\hline
\end{tabular}

The research work described in this thesis was carried out at the Catalytic Processes and Materials (CPM) and the Physics of Fluids (PoF) groups at the MESA+ Institute for Nanotechnology and Faculty of Science and Technology, University of Twente, P.O. Box 217, 7500 AE, Enschede, The Netherlands.

The project was financially supported by MESA+ Institute for Nanotechnology.

Nederlandse titel: Groei en bevochtigingseigenschappen van koolstof nanodraden Author: Hrudya Nair [Email: hrudyanair2011@gmail.com] Cover design: Hrudya Nair, K. Vijayakumaran Nair, Bert Geerdink Printing: Gildeprint Drukkerijen, Enschede, The Netherlands

\section{Copyright (C) 2014 by Hrudya Nair}

All rights reserved. No part of this document may be reproduced or transmitted, in any form or by any means, including, but not limited to electronic, mechanical, photocopying, recording, or otherwise, in whole or in part without the prior written permission from the copyright holder.

ISBN: 978-90-365-3786-5 


\section{GROWTH AND WETTING PROPERTIES \\ OF \\ CARBON NANOFIBERS}

\section{DISSERTATION}

to obtain

the degree of doctor at the University of Twente, on the authority of the rector magnificus,

Prof. Dr. H. Brinksma

on account of the decision of the graduation committee,

to be publicly defended

on Thursday, 29 January 2015 at 14:45 h

by

Hrudya Nair

born on 01 December 1983

in Koothattukulam, Kerala, India. 
This dissertation has been approved by the promoters:

Prof. Dr. Ir. Leon Lefferts

$\&$

Prof. Dr. Rer. Nat. Detlef Lohse

and the assistant-promoter:

Dr. Arie van Houselt 
To the Almighty, my teachers and family,

$$
\text { especially }
$$

sister Dr. Harsha Nair, brother-in-law Vivek P. R.,

$$
\text { husband V. Abhijit and }
$$

mom Shyla Nair and dad K. Vijayakumaran Nair. 



\section{CONTENTS}

1. Introduction: Wettability and Carbon Nanofibers

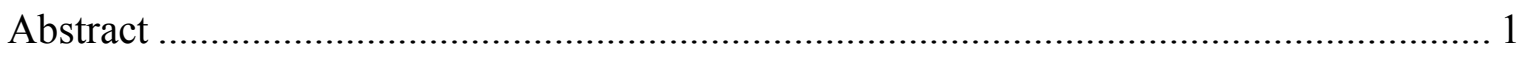

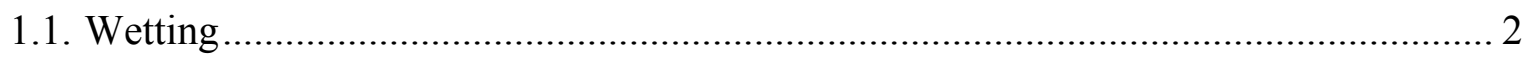

1.2. Wetting and Catalysis ...................................................................................... 4

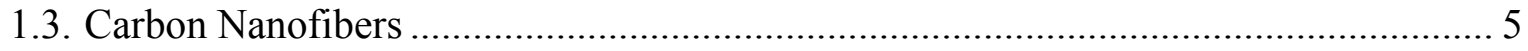

1.4. Scope of this thesis................................................................................... 7

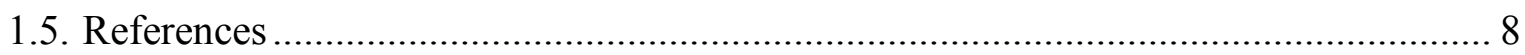

2. An introduction to wetting: Absence of an evaporation-driven 11-28 wetting transition on omniphobic surfaces

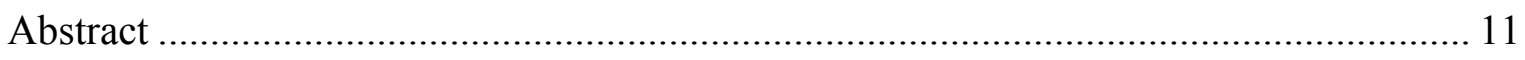

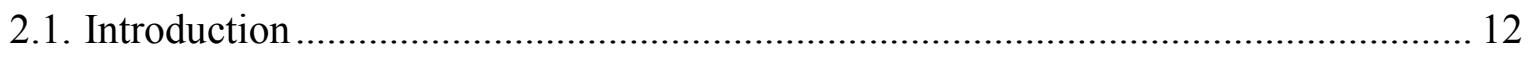

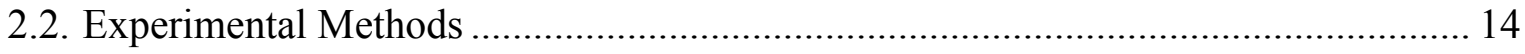

2.2.1. Fabrication of microstructures by photolithography and reactive ion etching. 14

2.2.2. Coating and contact angle measurement ....................................................... 15

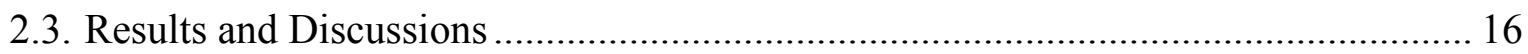

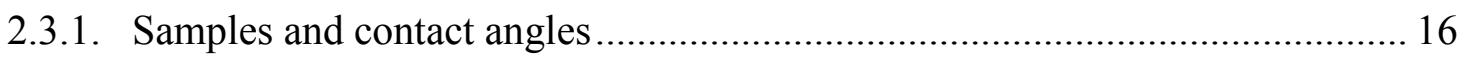

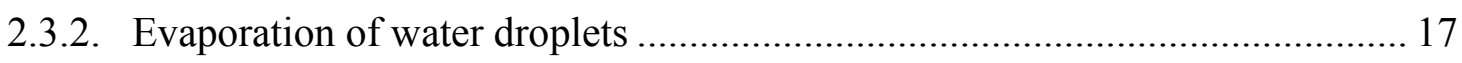

2.3.3. Evaporation of a colloidal solution ............................................................ 20

2.3.4. Comparing with mechanisms from literature ................................................... 21

2.3.5. The global energy argument crisis ............................................................. 25

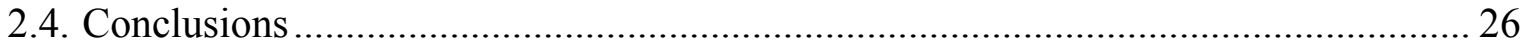

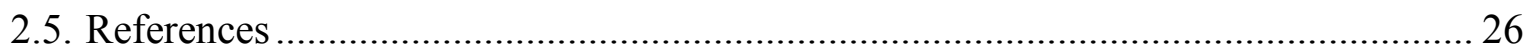


3. Influence of thin film nickel pretreatment on catalytic thermal $29-46$ chemical vapor deposition of carbon nanofibers

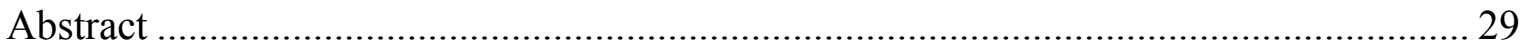

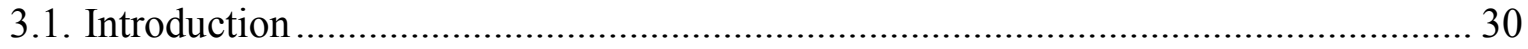

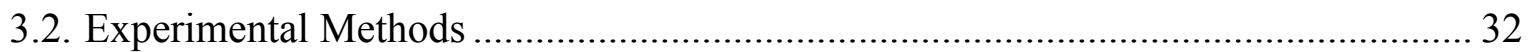

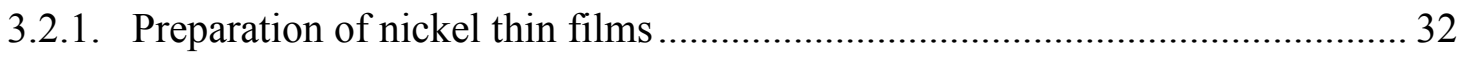

3.2.2. Pretreatment for the formation of nanoparticles............................................. 33

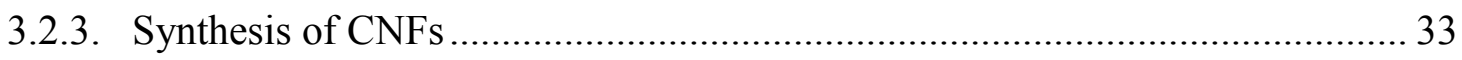

3.2.4. Characterization of nanoparticles and CNFs ................................................. 33

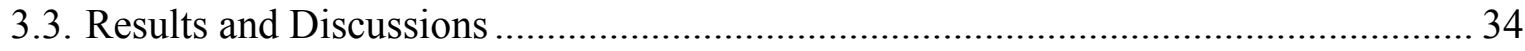

3.3.1. Pretreatment and Ni nanoparticle formation .................................................. 34

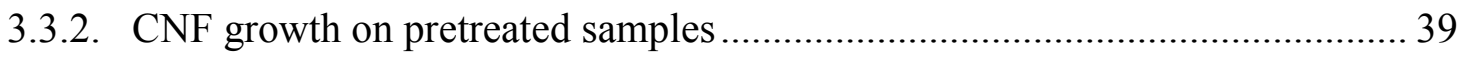

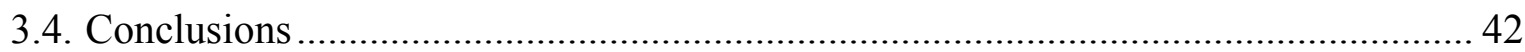

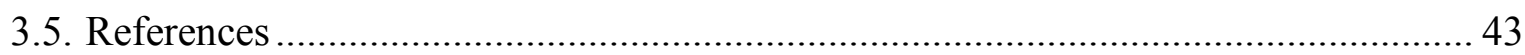

4. A Raman and infrared study of carbon nanofiber growth 47-62

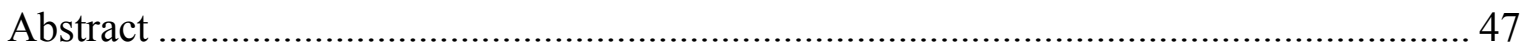

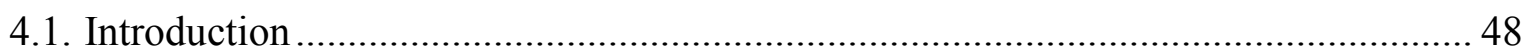

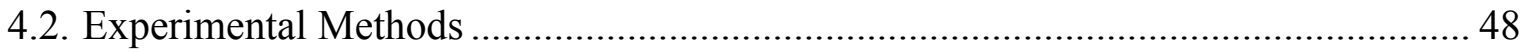

4.2.1. Preparation of nickel thin films on oxidized silicon substrates ....................... 48

4.2.2. Synthesis of CNFs on Ni-coated oxidized silicon substrates .......................... 49

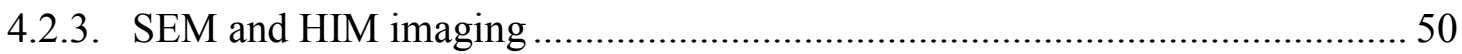

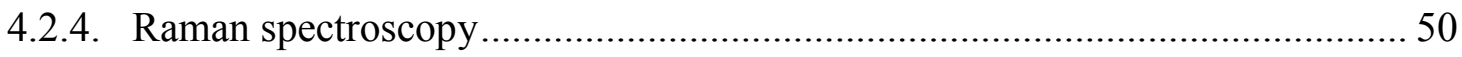

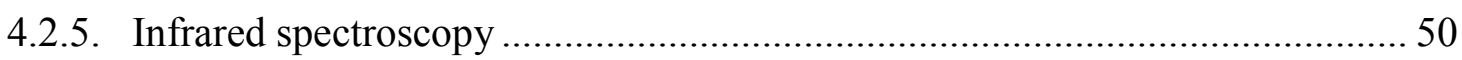

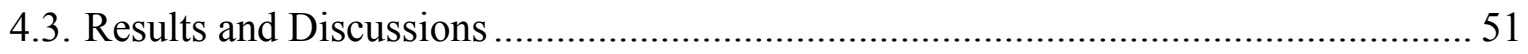

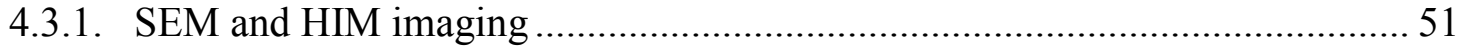

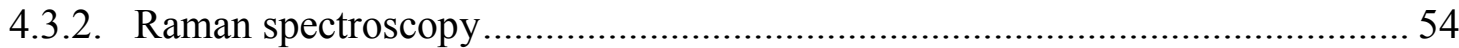

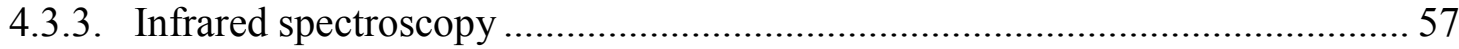

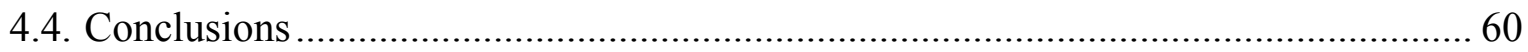

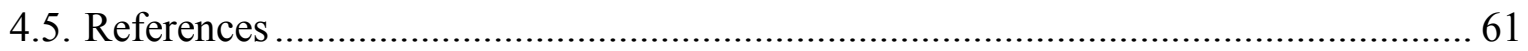


5. Evidence of wettability variation on carbon nanofiber layers grown on oxidized silicon substrates

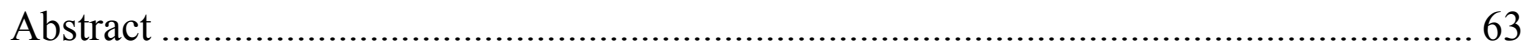

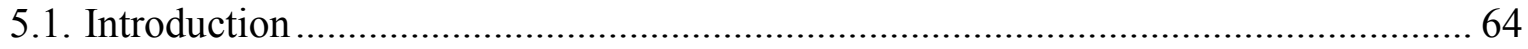

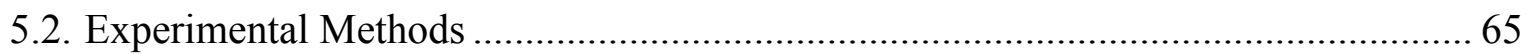

5.2.1. Preparation of nickel-based thin films on oxidized silicon substrates ............. 65

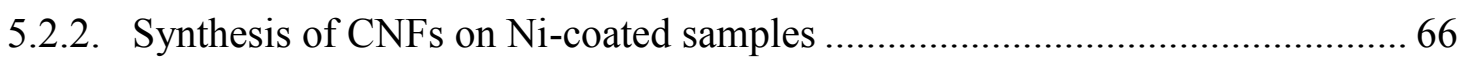

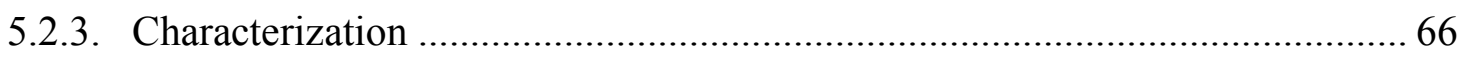

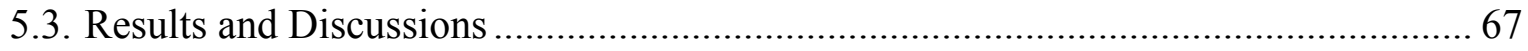

5.3.1. Influence of pretreatment atmosphere on CNF synthesis ............................... 67

5.3.2. Influence of hydrogen on CNF synthesis ...................................................... 71

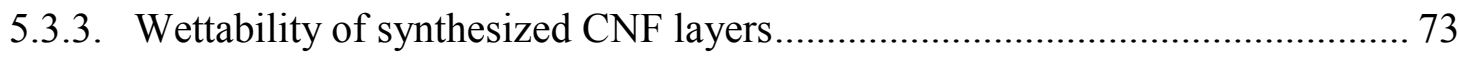

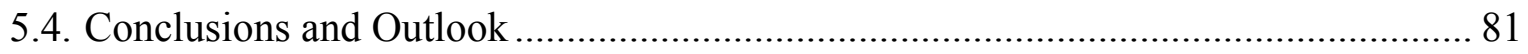

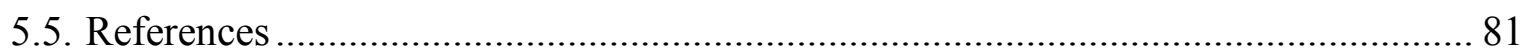

6. How water droplets evaporate on a superhydrophobic CNF 85-100 substrate

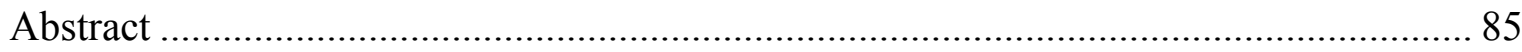

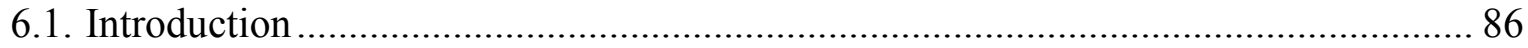

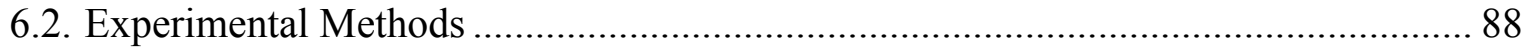

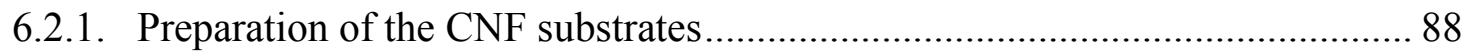

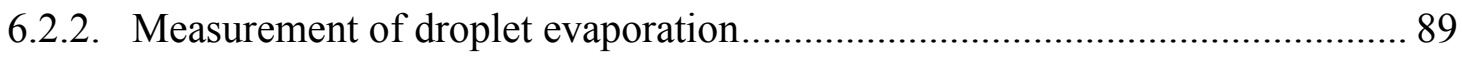

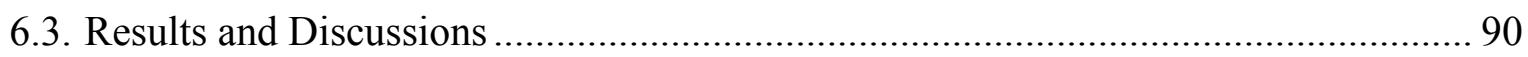

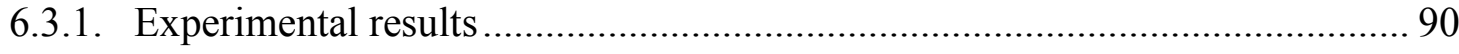

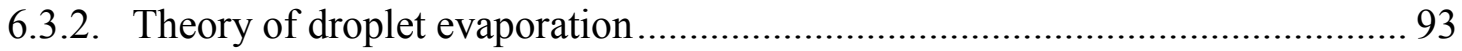

6.3.3. Comparison between theory and experiment .................................................... 96

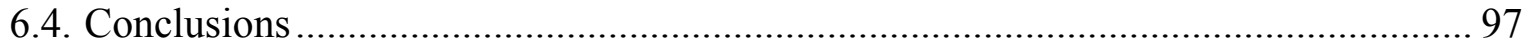

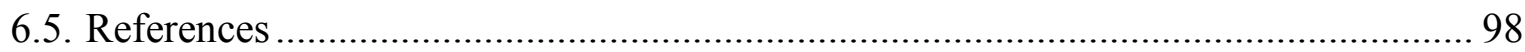


7. The Leidenfrost temperature increase for impacting droplets 101-118 on carbon nanofiber surfaces

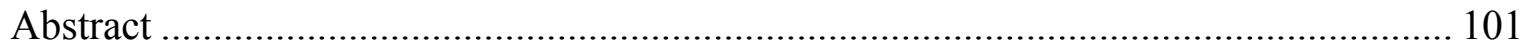

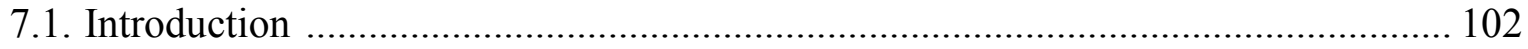

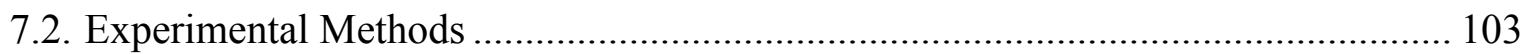

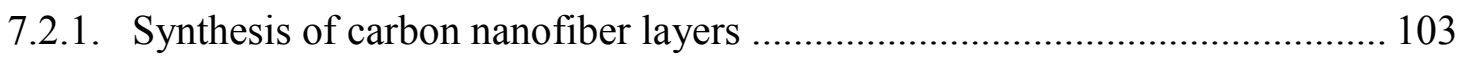

7.2.2. FC-72 droplet impact experiments on CNF layers........................................ 106

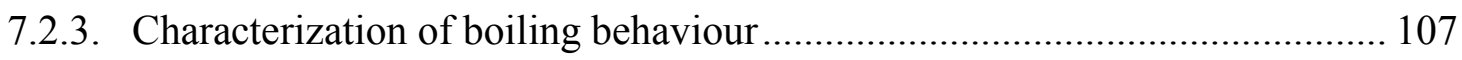

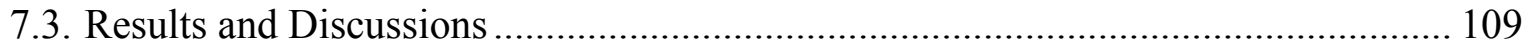

7.3.1. Dynamic Leidenfrost temperature ................................................................ 109

7.3.2. Estimate of the relevant time scales ........................................................... 112

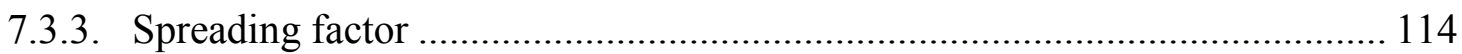

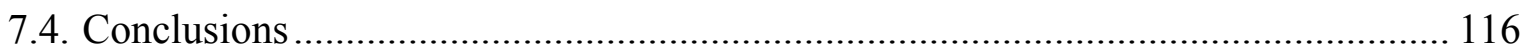

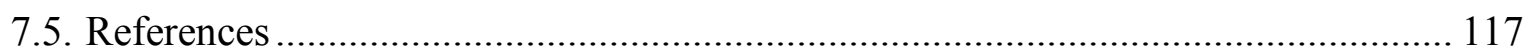

8. Summary and Outlook $119-125$

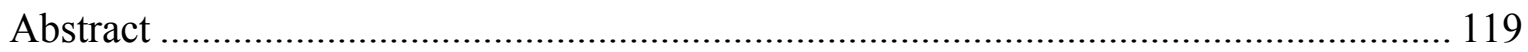

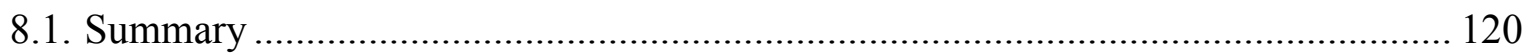

8.2. General Recommendations and Outlook …............................................................ 123

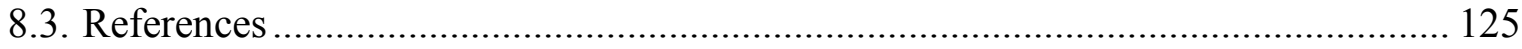

Samenvatting in het Netherlands (Summary in the Dutch) ...........................................i-iv

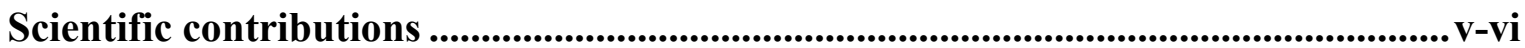

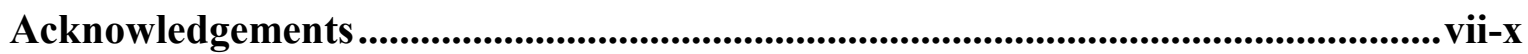

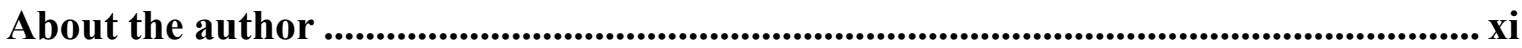

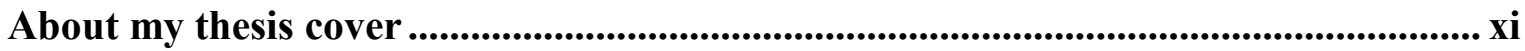




\section{Chapter 1}

\section{Introduction: Wettability and carbon nanofibers}

\section{Abstract}

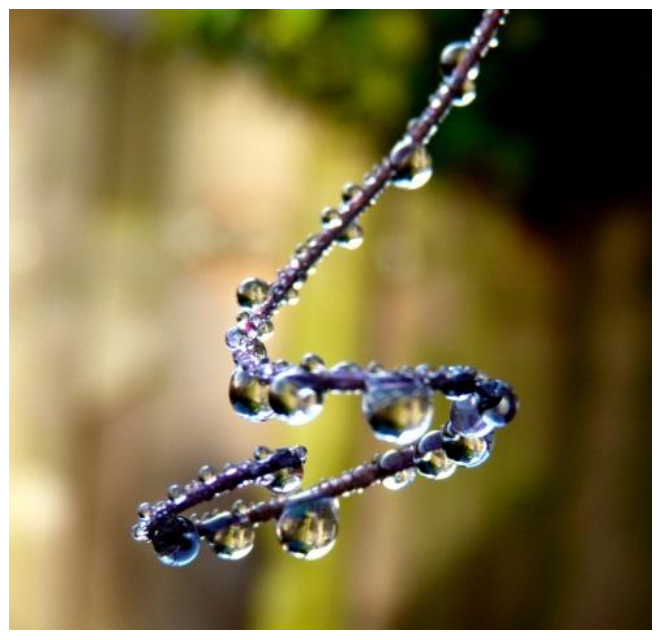

In this thesis we investigate the growth and wetting properties of carbon nanofibers (CNFs). In this chapter we introduce the concepts of wettability and a short description of the history, growth and characteristics of CNFs is given. Their unique structural and wetting properties, investigated in this thesis, render them an interesting candidate for application in microfluidics. 


\section{$1.1 \quad$ Wetting}

Surface wettability is of interest not only from the fundamental viewpoint, but also because of its technological applications in, for example, areas as coatings, textiles, lubrication and microfluidic technology. ${ }^{[1]}$ In particular superhydrophobicity has attracted substantial research interest since Barthlott and Neinhuis ${ }^{[2]}$ examined the microscopic origin of the high hydrophobicity of the lotus leaf (see Figure 1). Superhydrophobicity is characterized by a macroscopic water contact angle $\theta$ larger than $150^{\circ}$, combined with small sliding angles and a low hysteresis between advancing and receding contact angles. ${ }^{[3]}$

Droplets on chemically heterogeneous or microstructured surfaces can generally adopt two different states: the Wenzel state, in which the liquid completely wets the entire surface ${ }^{[4]}$ (see Figure 2a), or the Cassie-Baxter state, in which the droplet only partly wets the surface, leaving air in between the microstructures under the droplet ${ }^{[5]}$ (see Figure $2 \mathbf{b}$ ).

Wenzel proposed that the apparent (macroscopic) droplet contact angle $\theta^{*}$ in case of complete wetting of the microstructured surface is influenced by the increase of the wetted area (compared to a droplet on a similar flat surface),

$$
\cos \theta^{*}=r \cos \theta
$$

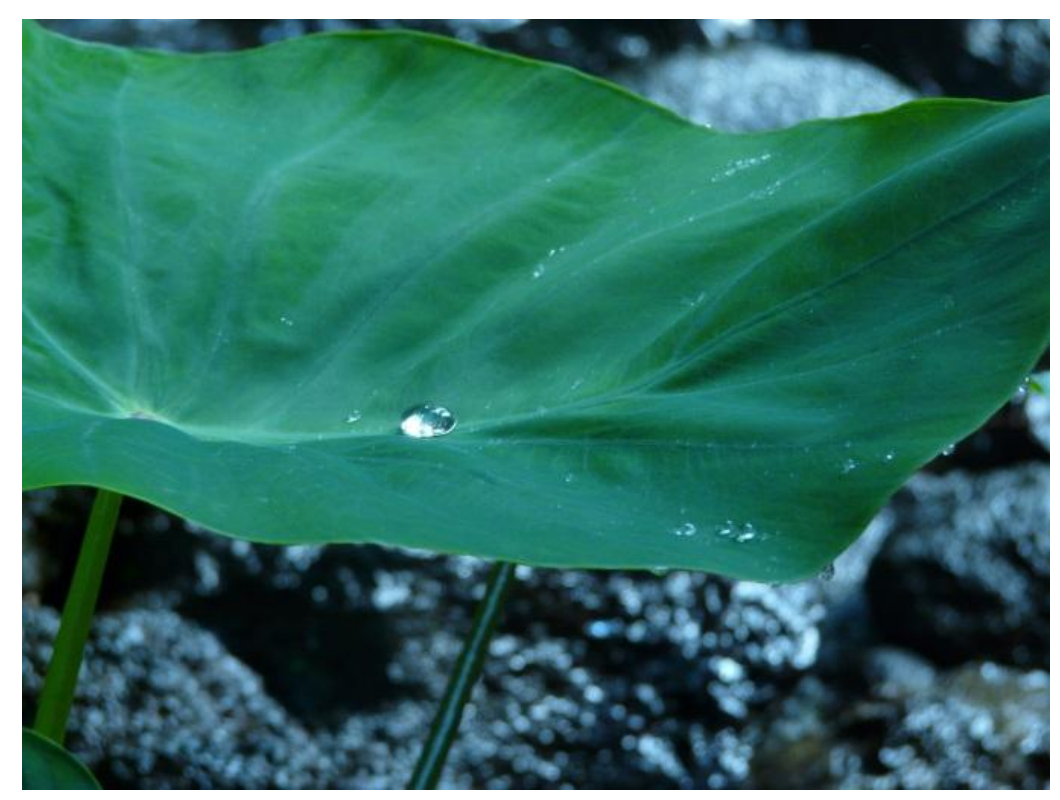

Figure 1: Photograph of water droplets on a lotus leaf (ㄷ Dr. Arie van Houselt). 
where $r$ is the ratio of the wetted surface to the projected flat surface under the droplet and $\theta$ is the equilibrium contact angle on a flat, homogeneous surface, given by Young's equation ( $\cos \theta=\frac{s v^{-} s l}{l v}$, with the interface tension between the solid $(s)$, liquid ( $l$ ) and vapour (v) phase). Equation (1) implies that in the Wenzel state the inherent wettability of the corresponding flat surface is enhanced with an increase in surface roughness (see Figure 2c).

In the Cassie-Baxter state equation (1) changes to:

$$
\cos \theta^{*}=-1+{ }_{s}(1+\cos \theta)
$$

where $s$ is the fraction of the liquid interface that is in contact with the superhydrophobic solid. For droplets in the Cassie-Baxter state the apparent contact angle always increases upon introduction of surface roughness (see Figure 2d).

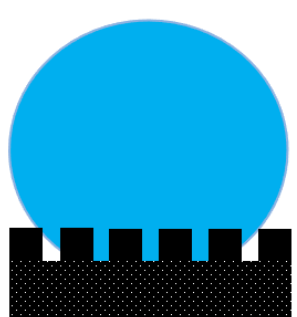

(a) Wenzel State

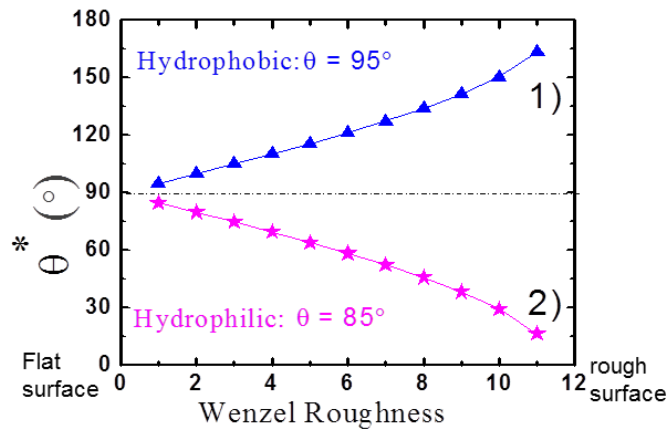

(c) Wenzel model

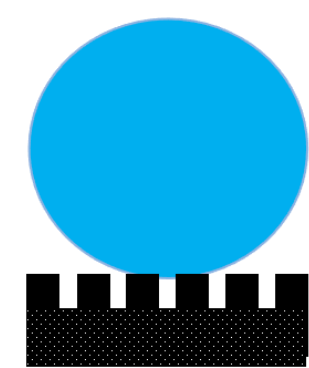

(b) Cassie-Baxter State

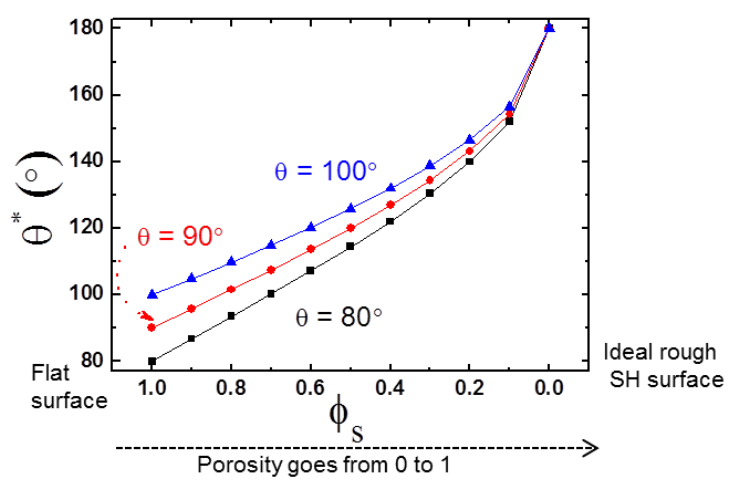

(d) Cassie-Baxter model

Figure 2: A schematic representation of a liquid droplet in (a) the Wenzel state and (b) the Cassie-Baxter. In (c) and (d) the apparent contact angles upon introduction of surface roughness according to the Wenzel (c) and the Cassie-Baxter model (d) are shown. 


\subsection{Wetting and Catalysis}

This thesis is the result of fruitful co-operation between a research group focused on catalysis and a research group focused on fluid physics. The common interest raised due to the interesting properties of (super)hydrophobic surfaces and their possible applications.

At a (super)hydrophobic liquid-solid interface the effective liquid-solid contact area is reduced by the entrapped vapor, which ultimately enhances slip and reduces drag and related energy dissipations. ${ }^{[6]}$ Drag reduction is of importance for chemical engineering applications like the reactants or products flows in the pipelines of chemical production units, where pressure losses are substantial particularly if dimensions become smaller. Drag reduction is particularly important for fluid flow in microfluidic and nanofluidic systems, which are distinguished by a large surface-tovolume ratio and flow at small Reynolds, capillary, and Bond numbers. ${ }^{[7,8]}$ This created a tremendous interest in the design of anti-wetting surfaces, which are recently used to, among others, enhance mixing, slippage, drug delivery, heat transfer surfaces in air conditioners and to enhance the efficiency of catalytic microreactors. ${ }^{[9-11]}$ For even the larger membrane reactors, it is demonstrated that the selective hydrophobization of the membrane may drastically enhance the performance of a gasliquid-solid microreactor. ${ }^{[12]}$

Due to their excellent chemical and mechanical stability carbon nanofibers (CNFs) are a promising catalyst support and they could themselves be active as oxidation or, after nitrogen incorporation as base catalysts. ${ }^{[13]}$

The wettability of the support materials may be crucial in catalytic reactions hydrophilicity is preferred for reactions in aqueous media whereas hydrophobicity is preferred for reactions in non-aqueous media, where water is an important by-product. Hydrophobicity of the support enhanced the catalytic hydrogenation of nitrobenzene and dominated over the effect of catalyst size and loading. ${ }^{[14]}$

Hydrophobic micro-porous surfaces with high surface to volume ratio can replace the gas diffusion layer in fuel cells and can increase their performance by 
effectively removing water and improving the oxygen transport, thereby decreasing the overall mass and complexity and increasing the portability of a fuel cell. ${ }^{[15,16]}$

In this thesis wetting and catalysis are brought together in the (catalytic) growth and evaluation of the wetting properties of CNFs.

\subsection{Carbon Nanofibers}

CNFs, probably first described in $1889^{[17]}$ have been researched profoundly over the years. Robertson ${ }^{[18]}$ reported the formation of graphitic carbon from methane in the presence of metal catalysts at relatively lower temperatures. A few years later Baker et al. detailed the formation of nanostructured carbon using the supported transition metal catalysts Nickel (Ni), Cobalt (Co) and Iron (Fe). ${ }^{[19]}$ Till the eighties of the twentieth century detailed studies of CNFs were merely motivated by the undesirable deposition of carbon on the surface of steam crackers in the production of olefins. $^{[20]}$ The last decades three discoveries have boosted the research of nanostructured carbon. Firstly, the discovery of buckminsterfullerene, $\mathrm{C}_{60}$, in 1985 by, Kroto, Curl and Smalley. ${ }^{[21]}$ Secondly the synthesis of carbon nanotubes (CNTs) in 1991 by Iijima $^{[22]}$. Lastly, and most spectacularly, the discovery of graphene, a single graphite sheet consisting of a hexagonal network of $\mathrm{sp}^{2}$ hybridized carbon atoms, in 2004 by Novoselov and Geim. ${ }^{[23]}$

CNFs are filamentous nanostructures grown by the diffusion of carbon through transition metal catalysts and the subsequent precipitation as graphitic filaments. The CNFs used in this thesis are grown by chemical vapor deposition (CVD) using ethylene $\left(\mathrm{C}_{2} \mathrm{H}_{4}\right)$ as carbon containing gas and $\mathrm{Ni}$ as catalyst. A photograph of the CVD setup is shown in Figure 3.

The view held in literature is that CVD-synthesized carbon nanostructures are formed by a solution-diffusion-precipitation process that generates graphitic carbon. ${ }^{[19]}$ Hydrocarbon molecules decompose at the surface of the catalyst nanoparticle, and the carbon atoms dissolve into the metal forming a solid solution. Upon super-saturation of the catalyst particle, carbon growth occurs by diffusiondriven precipitation of graphite layers at the surface of the particle. Solute precipitation occurs preferentially at dislocations and grain boundaries (i.e. where 


\section{Chapter 1}

stress fields are more intense), as a consequence of which polycrystalline metal nanoparticles offer many sites for precipitation of carbon and nanostructure growth. Tip-type and base-type growth mechanisms are reported, referring to the position of the catalyst particle during synthesis. The diameter and formation rate of carbon nanostructures highly depends on the feed gas composition, temperature and the type of metallic catalyst.

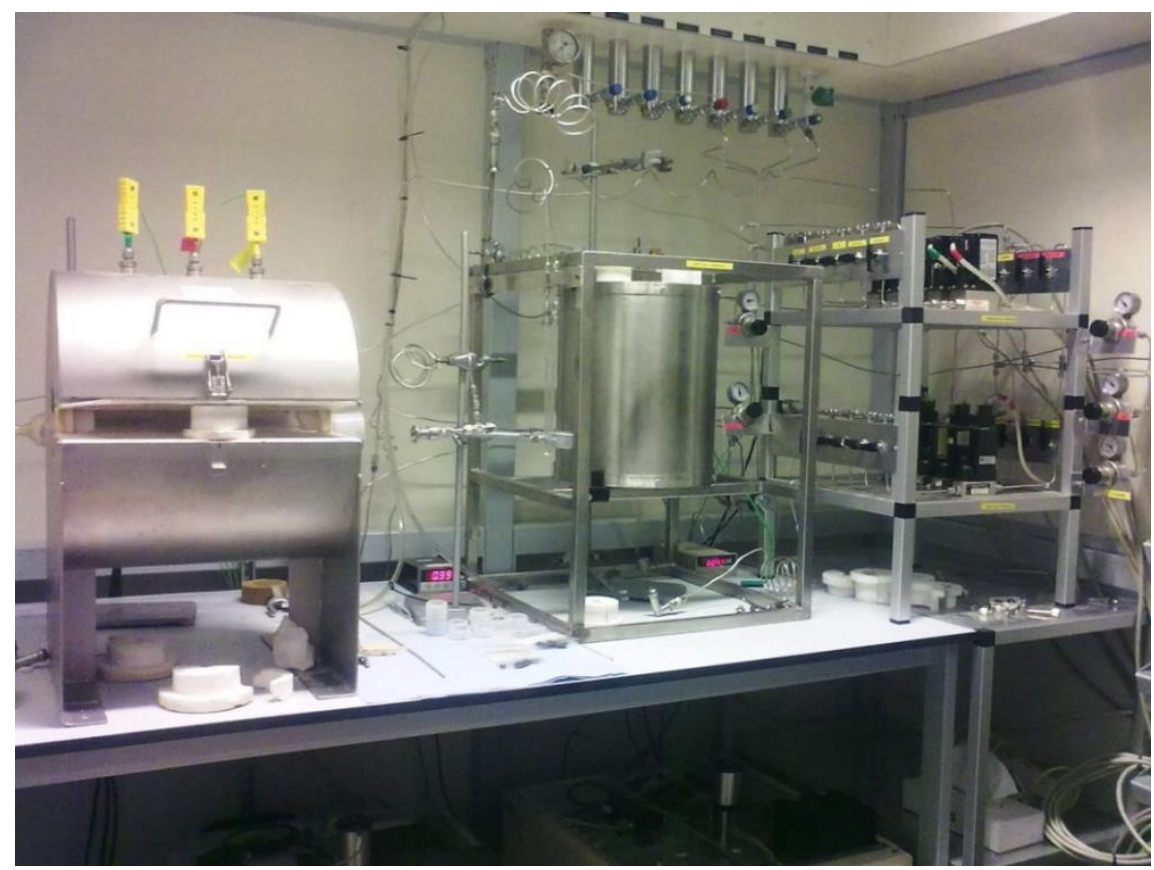

Figure 3: A photograph of the CVD setup for carbon nanofiber growth.

A graphene sheet rolled up into a cylinder is called a single walled carbon nanotube (CNT), while multi-walled nanotubes (MWNT) consist of multiple rolled layers (concentric tubes) of graphene. CNFs are classified based on the axis $\alpha$ between the graphene sheets and the central fiber axis as either platelet-type $\left(\alpha \sim 90^{\circ}\right)$ or fishbone type $\left(\alpha \sim 45^{\circ}\right)$. The anisotropy of graphite influences the properties of carbon nanostructures. CNTs have graphitic basal planes exposed with very few chemically active defect sites; whereas CNFs have hydrogen terminated graphitic edges, which are more amenable to chemical modification.

Carbon nanostructures are mostly studied by scanning electron microscopy (SEM), transmission electron microscopy (TEM), energy-dispersive X-ray (EDX) spectroscopy and Raman spectroscopy. ${ }^{[24,25]}$ 


\subsection{Scope of this thesis}

The subject of this thesis is the direct synthesis of well-adhesive carbon nanofiber (CNF) layers by thermal catalytic chemical vapor deposition method, with controllable morphology and wettability and a uniform substrate coverage on thin films of nickel on silicon substrates. The wetting properties of CNFs are evaluated via interaction with static droplets (water droplet evaporation under ambient conditions) and dynamic droplets (FC-72 and water droplet impact on heated CNF surfaces at different Weber numbers) droplets. For comparison, wettability studies on flat silicon surfaces are also presented.

Chapter 2 introduces the concept of wettability, using chemically coated micro-textured silicon surfaces (fabricated by photolithography), which are omniphobic (water and oil repellant). Their stability against a so-called wetting transition from the Cassie-Baxter into the Wenzel state during evaporation of millimetric water droplets is investigating by varying the shape, surface roughness and edge curvature of the micropillars on the silicon surface. The experimental findings are compared with existing models that describe the Cassie-Baxter to Wenzel transition.

In Chapter 3 we discuss the fabrication of nickel thin film coated silicon substrates for the synthesis of CNFs. Various substrates configurations (10 nm Ni; 25 $\mathrm{nm} \mathrm{Ni} ; 25 \mathrm{~nm} \mathrm{Ni} / 10 \mathrm{~nm} \mathrm{Ta}$ on $\mathrm{SiO}_{2}$ ) are described and the formation of $\mathrm{Ni}$ nanoparticles from the deposited continuous thin films and the resulting CNF growth are studied. The varied parameters are the substrate configuration, pretreatment atmospheres (vacuum, nitrogen, air and hydrogen), pretreatment temperature and time.

In Chapter 4 the growth of carbon nanofibers on hydrogen-pretreated oxidized silicon substrates for different synthesis times is studied using Raman and Infrared spectroscopy, high resolution scanning electron microscopy and Helium Ion Microscopy, for various growth times using ethylene and ethylene/hydrogen as the hydrocarbon source.

Typically superhydrophobicity on CNF substrates is obtained by chemical 
post-treatment of the surface. Chapter 5 describes the direct synthesis of welladhesive CNF surfaces, on oxidized silicon substrate, with complete surface coverage as well as tunable wettability, without the necessity of further chemical post-synthesis treatments, for applications in silicon-based microfluidic systems.

In Chapter 6 the time evolution of the water contact angle during evaporation under ambient conditions $\left(\mathrm{T} \sim 23^{\circ} \mathrm{C}, \mathrm{H} \sim 0.3\right)$ is studied. The contact angle and droplet mass during evaporation of water droplet are examined and the experimental data is compared with theoretical models.

In Chapter 7, we discuss the boiling behavior (contact boiling and film boiling) and dynamic Leidenfrost temperature (the transition from contact boiling to film boiling for different Weber numbers) of impacting drops on heated silicon and CNF surfaces. The transition from the contact boiling to the film boiling regime depends not only on the temperature of the surface and the kinetic energy of the droplet, but also on the size of the structures fabricated on the surface. We experimentally show that surfaces covered with CNFs delay the transition to film boiling to much higher temperatures compared to smooth surfaces. We present physical arguments showing that, because of the small scale of the carbon fibers, they are cooled by the vapor flow just before the liquid impact, thus permitting contact boiling up to much higher temperatures than on smooth surfaces.

\subsection{References}

[01] P. de Gennes, F. Brochard-Wyart, D. Quéré, Capillarity and Wetting Phenomena - Drops, Bubbles. Pearls, Waves, Springer-Verlag New York, Inc., 2004.

[02] W. Barthlott and C. Neinhuis, Planta, 1997, 202, 1.

[03] D. Bonn, J. Eggers, J. Indekeu, J. Meunier, and E. Rolley, Rev. Mod. Phys., 2009, 81, 739 .

[04] R. N. Wenzel, Ind. Eng. Chem., 1936, 28, 988.

[05] A. D. B. Cassie and S. Baxter, Trans. Faraday Soc., 1944, 40, 546.

[06] D. Quéré, Annu. Rev. Mater. Res., 2008, 38, 71.

[07] H. A. Stone, A. D. Stroock, and A. Ajdari, Annu. Rev. Fluid Mech., 2004, 36, 381. 
[08] D. J. Beebe, G. A. Mensing, and G. M. Walker, Annu. Rev. Biomed. Eng., 2002, 4, 261.

[09] J. P. Rothstein, Annu. Rev. Fluid Mech., 2010, 42, 89.

[10] R. S. Voronov, D. V. Papavassiliou, and L. L. Lee, Ind. Eng. Chem. Res., 2008, 47, 2455

[11] Y. Zhang, H. Xia, E. Kim, and H. Sun, Soft Matter, 2012, 8, 11217

[12] H. C. Aran, J. K. Chinthaginjala, R. Groote, T. Roelofs, L. Lefferts, M. Wessling, and R. G. H. Lammertink, Chem. Eng. J., 2011, 169, 239.

[13] J. H. Bitter, J. Mater. Chem., 2010, 20, 7312.

[14] M. S. Hoogenraad, M. F. Onwezen, A. J. van Dillen, J. W. Geus, Stud. Surf. Sci. Catal., 1996, 101, 1331.

[15] P. A. Stuckey, J. F. Lin, A.M. Kannan, and M. N. Ghasemi-Nejhad, Fuel cells, 2010, 10(3), 369.

[16] Q. Duan, B. Wang, J. Wang, H. Wang, and Y. Lu, J. Power Sources, 2010, 195, 8189.

[17] T.V. Hughes and C.R. Chambers, Manufacture of Carbon Filaments, 1889, US Patent No. 405, 480.

[18] S. D. Robertson, Nature, 1969, 221, 1044.

[19] R. T. K. Baker, M. A. Barber, P. S. Harris, F. S. Feates, and R. J. Waite, J. Catal., 1972, 26, 51.

[20] K. P. de Jong and J. W. Geus, Catal. Rev.- Sci. Eng., 2000, 42(4), 48.

[21] H. W. Kroto, J. R. Heath, S. C. O’Brien, R. F. Curl, and R. E. Smalley, Nature, 1985, 318, 162.

[22] S. Iijima, Nature, 1991, 354, 56.

[23] K. S. Novoselov, A. K. Geim, S. V. Morozov, D. Jiang, Y. Zhang, S. V. Dubonos, I. V. Grigorieva, and A. A. Firsov, Science, 2004, 306, 666.

[24] K. L. Klein, A. V. Melechko, T. E. McKnight, S. T. Retterer, P. D. Rack, J. D. Fowles, D. C. Joy, and M. L. Simpson, J. Appl. Phys., 2008, 103, 061301.

[25] M. S. Dresselhaus, G. Dresselhaus, R. Saito, and A. Jorio, Phys. Rep., 2005, 409, 47. 



\section{Chapter 2}

\section{An introduction to wetting $1{ }^{1}$}

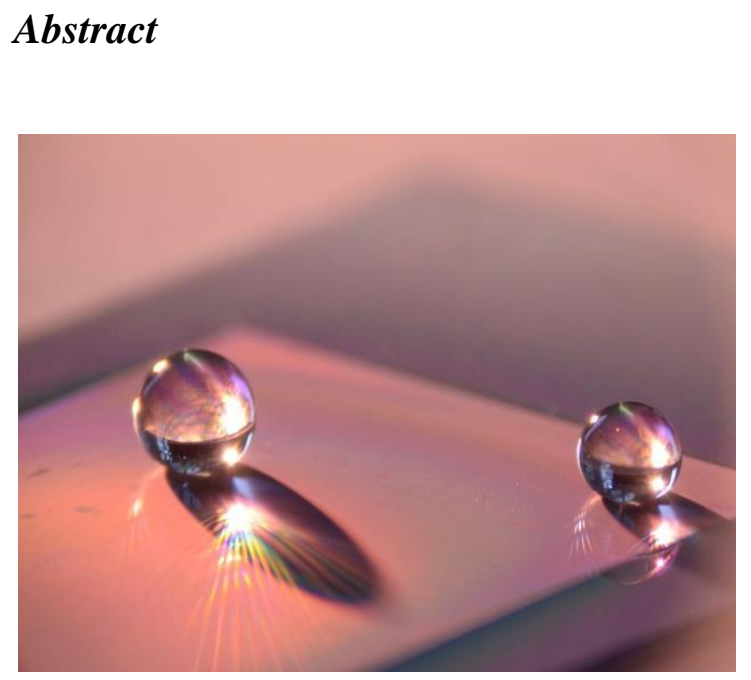

Surfaces that exhibit contact angles close to $180^{\circ}$ for both polar and nonpolar solvents are rare. Here, we report the fabrication of such "omniphobic" surfaces by photolithography. We investigate their stability against a socalled wetting transition during evaporation of millimetric water droplets by systematically varying the shape and surface roughness of the micropillars on the surface. We show that a low edge curvature of the top of the micropillars strongly delays the transition, while it completely disappears when the surface roughness is increased. We compare these experimental findings with existing models that describe the Cassie-Baxter to Wenzel transition and conclude that new models are needed which include the hurdle of an energy barrier for the wetting transition. Our results reveal that by increasing the roughness of the micropillars, we do not affect the apparent equilibrium contact angle of the droplets. The dynamic robustness of the surface is, however, dramatically enhanced by an increase of the surface roughness.

\footnotetext{
1 This chapter is published as "Absence of an evaporation-driven wetting transition on omniphobic surfaces” by A. Susarrey-Arce, Á. G. Maŕin, H. Nair, L. Lefferts, J. G. E. Gardeniers, D. Lohse and A. van Houselt, Soft Matter, 2012, 8, 9765.

* Microstructures fabricated by A. Susarrey-Arce; Experiments performed together with A.G. Marin.
} 


\section{$2.1 \quad$ Introduction}

Superhydrophobic surfaces are extremely water repellent, ${ }^{[1]}$ and contact angles above $150^{\circ}$ have been reported. Inspiration for artificial superhydrophobic surfaces is found in nature: lotus leaves, ${ }^{[2]}$ gecko feet, ${ }^{[3]}$ and the legs of the water strider ${ }^{[4]}$ are natural superhydrophobic surfaces. A myriad of applications of superhydrophobic surfaces have been reported, including self-cleaning, ${ }^{[5]}$ drag-reducing, ${ }^{[6,7]}$ antifreezing ${ }^{[8]}$ and selective condensation surfaces. ${ }^{[9]}$

Surfaces which are not only superhydrophobic, but also exhibit high contact angles $\left(>150^{\circ}\right)$ with other liquids than water, like oils and alkanes, are even more intriguing. Oil-repelling surfaces are known as oleophobic surfaces and are, for example, used as coatings to repel oily fingerprints on the screens of smartphones. Surfaces which combine superhydrophobicity and superoleophobicity are referred to as "omniphobic". However omniphobic surfaces are rare. ${ }^{[10,12-15]}$

Superhydrophobic surfaces usually combine a low surface energy with surface texture, comprised of microstructures. The influence of the surface microstructures on the apparent contact angle of droplets is usually explained by the Wenzel model, ${ }^{[16]}$ when the liquid fully fills the space between the surface microstructures, or by the Cassie-Baxter model, ${ }^{[17]}$ when the liquid lays on top of the microstructures, leaving air in between the microstructures under the droplet. In the case of complete wetting, Wenzel proposed that the apparent (macroscopic) droplet contact angle $\theta^{*}$ is influenced by the increase of the wetted area (compared to a droplet on a flat surface),

$$
\cos \theta^{*}=r \cos \theta
$$

where $r$ is the ratio of the wetted surface to the projected flat surface under the droplet and $\theta$ is the equilibrium contact angle on a flat, homogeneous surface, given by Young's equation $\left(\cos \theta=\frac{s v^{-} s l}{l v}\right.$, where is the interface tension between the solid (s), liquid (1) and vapour (v) phase). Usually, the contact angle hysteresis is high in the Wenzel state (hereafter W), due to the strong contact-line pinning at the microstructures. ${ }^{[1]}$ 
In contrast, when the droplet sits on a composite surface of air and a hydrophobic solid, Cassie and Baxter derived an equation for the apparent (macroscopic) droplet contact angle, $\theta^{*}$ :

$$
\cos \theta^{*}=-1+{ }_{s}(1+\cos \theta)
$$

where $s$ is the fraction of the liquid interface that is in contact with the superhydrophobic solid. In the Cassie-Baxter or "Fakir" state (hereafter CB), droplets can easily roll off, often referred to as "self-cleaning". ${ }^{[18]}$ However, it has been found $^{[19]}$ that none of the above-mentioned equations describes the actual contact angle generally correctly.

The CB state is commonly accepted to be metastable ${ }^{[1]}$ and there have been several recent reports on the $\mathrm{CB}$ to $\mathrm{W}$ wetting transitions in droplets on microstructured surfaces. ${ }^{[20-33]}$ The $\mathrm{CB}$ to $\mathrm{W}$ transition can occur spontaneously, ${ }^{[20-23]}$ or can be triggered by, for instance, rapid deceleration, ${ }^{[23]}$ application of an electrical voltage, ${ }^{[24]}$ drop impact, ${ }^{[25,26]}$ vibration of the substrate, ${ }^{[27,28]}$ droplet squeezing, ${ }^{[29]}$ or evaporation $^{[30-32]}$. On the other hand, the $\mathrm{CB}$ to $\mathrm{W}$ transition is not always observed in evaporating droplets ${ }^{[34]}$. Reyssat et. al., ${ }^{[32]}$ for instance, reported that the $\mathrm{CB}$ to $\mathrm{W}$ transition does not occur on surfaces with arrays of high micropillars with aspect ratios $>10$. In order to model the experimental data for the evaporation-driven $\mathrm{CB}$ to $\mathrm{W}$ transition, two approaches are reported. One, in which the increase in the Laplacepressure inside the evaporating droplet causes the $\mathrm{CB}$ to $\mathrm{W}$ transition, was successfully tested for long and thin micropillars with relatively large mutual interspaces. $^{[32,35,36]}$ And the other, based on comparison of the global interfacial energies of the $\mathrm{CB}$ and the $\mathrm{W}$ state, ${ }^{[20-22,30,36-39]}$ which was successfully applied for shorter and thicker micropillars with relatively small mutual interspaces. ${ }^{[20-22,30]}$

Here, we report the fabrication of new superomniphobic surfaces, on which water droplets preserve the $\mathrm{CB}$ state their entire lifetime during evaporation. We compare this evaporation process to water droplet evaporation on superhydrophobic surfaces with nanostructures of similar dimensions, and we examine the applicability of the interfacial energy argument to describe the $\mathrm{CB}$ to $\mathrm{W}$ transition on these surfaces. 


\subsection{Experimental Methods}

\subsubsection{Fabrication of microstructures by photolithography and reactive ion etching}

The microarray was processed by reactive ion etching. In an attempt to control the angle of the sidewalls of the microstructures, the radio $(\mathrm{RF})$ power and $\mathrm{SF}_{6}, \mathrm{CHF}_{3}$ and $\mathrm{O}_{2}$ concentration were systematically varied. Etching experiments were performed in an Electrotech, Plasmafab 310-340 twin deposition/etch system, using a Silicon (100) wafer (100 mm diameter, $525 \mu \mathrm{m}$ thick, 5-10 $\Omega . c m$, p-type). The reactive ion etching is performed in a parallel plate system with an RF generator operating at $13.56 \mathrm{MHz}$ and an automatic matching network. The working temperature of the lower electrode $\left(10^{\circ} \mathrm{C}\right)$ was controlled with an oil bath. A uniform etching rate was maintained with a ceramic plasma shower. High $\mathrm{SF}_{6}$ concentrations lead to anisotropic Si etching. The additional dosing of $\mathrm{O}_{2}$ and $\mathrm{CHF}_{3}$ during the plasma reaction results in a higher isotropic etching rate, which smoothens the microstructures. The resulting nanostructures with a well-defined concave shape and homogenous sidewalls are shown in Figure 1A.

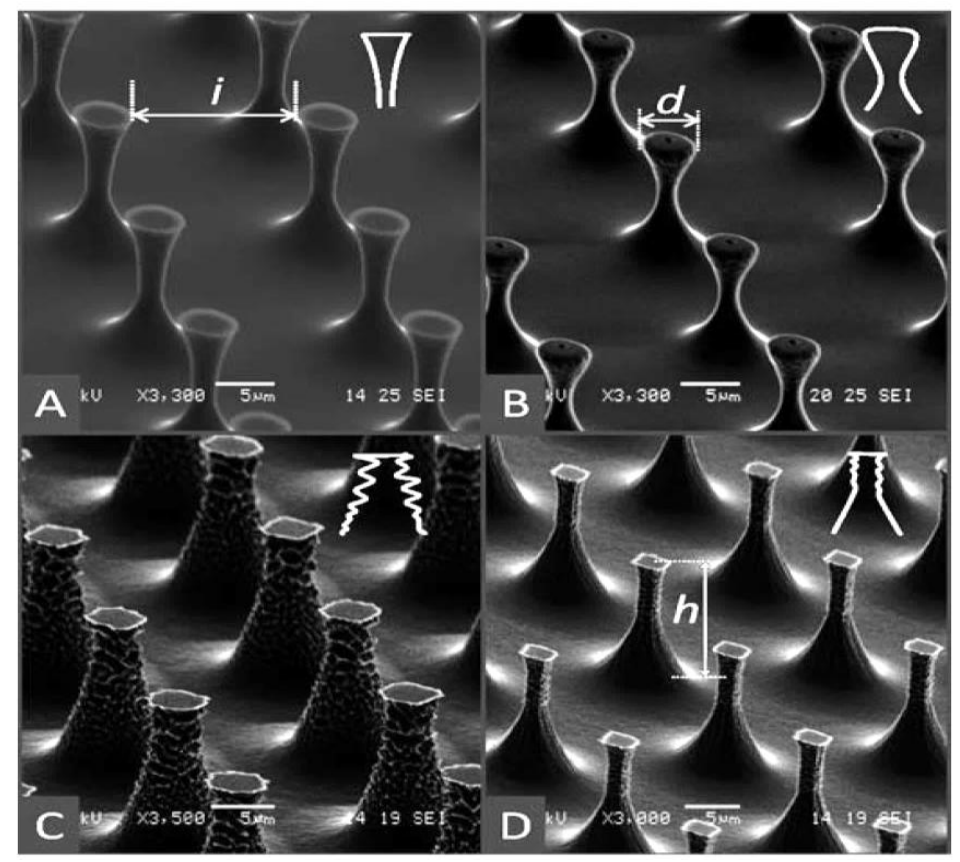

Figure 1: SEM micrographs of the surfaces A-D. In each image the scale bar represents $5 \mu \mathrm{m}$. The insets show a schematic representation of the micropillars. Straight lines represent smooth surfaces, while wavy lines represent rough surfaces. 
To increase the radius of curvature of the edge of the pillars, ca. $1 \mu \mathrm{m}$ of $\mathrm{SiO}_{2}$ was grown by wet thermal oxidation. Because silicon oxide occupies a ca. $40 \%$ larger volume than the original silicon, this oxidation procedure will lead to increase of radius of curvature of the pillar edges. Figure 1B shows a SEM image of the resulting microstructures. To increase the surface roughness of the pillar sides (Figure 1C and D) a different etching procedure was followed in an Adixen AMS100DE, with an RF generator at $13.56 \mathrm{MHz}$ and ICP plasma power up to $3 \mathrm{~kW}$. The temperature of the lower plate holder was kept at $-50{ }^{\circ} \mathrm{C}$, using liquid nitrogen as the coolant. The $\mathrm{SF}_{6}$ gas flow rate was maintained constant $(100 \mathrm{sccm})$, while the $\mathrm{O}_{2}$ flow rates were $20 \mathrm{sccm}$ and $25 \mathrm{sccm}$ for microstructures C and D. Subsequently, an extra step was performed for microstructures $\mathrm{D}$ : the $\mathrm{O}_{2}$ concentration in the plasma chamber was varied by pulses, from $25 \mathrm{sccm}$ to $15 \mathrm{sccm}$ during the etching process. The absence of $\mathrm{CHF}_{3}$ in the etching process resulted in an increase in the surface roughness. The resulting microstructures in Figure 1C exhibit a flat top-surface, with nanoflakes at the border, while the sides of these microstructures exhibit a porous structure. Figure 1D shows square micropillars with smaller diameters and porous sidewalls. Further details on the sample preparations are published elsewhere. ${ }^{[11]}$ The dimensions of the microstructures A-D are shown in Table 1.

\begin{tabular}{|c|ccc|}
\hline Microstructure & $h(\mu \mathrm{m})$ & $i(\mu \mathrm{m})$ & $d(\mu \mathrm{m})$ \\
\hline A & 8.8 & 14 & 3.6 \\
B & 7.8 & 14 & 4 \\
C & 9.2 & 13.5 & 4 \\
D & 8.8 & 14.5 & 2.4 \\
\hline
\end{tabular}

Table 1: Height $(h)$, pillar-to-pillar nearest neighbor interspace $(i)$ and diameter (d) of the microstructures on substrates A-D.

\subsubsection{Coating and contact angle measurement}

Silicon micropatterns were subsequently treated with $1 \%$ HF in water and $66 \%$ $\mathrm{HNO}_{3}$ in water before coating. Vapor deposition was carried out in a vacuum system using Trichloro (1H, 1H, 2H, 2H-perfluorooctyl) silane (FOTS 97\%, Sigma-Aldrich). Contact angle measurements during evaporation at room temperature $\left(21 \pm 1{ }^{\circ} \mathrm{C}\right)$ 
were realized at room temperature with a relative humidity of $35 \pm 5 \%$, placing a water droplet of 2-6 $\mathrm{L}$ on the microstructured substrate. The used water (18.2 $\mathrm{M} \Omega$ $\mathrm{cm})$ was purified in a Millipore Milli-Q system which involves reverse osmosis, ionexchange, and filtration steps. Side view videos were captured via CCD camera equipped with $420 \times$ magnifying lenses and with a recording time of 1-2 frames per second. The equilibrium contact angle on a FOTS-coated plane Si (001) wafer was $110^{\circ}$. The contact angle values on all samples were reproducible within three months after sample fabrication.

Evaporation experiments with drops containing a colloidal suspension of spherical polystyrene were carried out by allowing a water droplet containing polystyrene particles $(1 \mu \mathrm{m}$ diameter, initial concentration $0.08 \%$ weight and initial volume $5 \mu \mathrm{L}$ ) to evaporate on the superhydrophobic surface at room temperature and $30 \%$ of humidity. After a typical evaporation time of $45 \mathrm{~min}$, the solvent is completely evaporated and only the colloids are left upon the substrate.

\subsection{Results and Discussions}

\subsubsection{Samples and contact angles}

A photograph of droplets of n-octane ( $\left.l v=21.6 \mathrm{~m} \cdot \mathrm{N} \cdot \mathrm{m}^{-1}\right)$, water $\left(l v=72.8 \mathrm{~m} \cdot \mathrm{N} \cdot \mathrm{m}^{-1}\right)$ and olive oil $\left(l v=32.5 \mathrm{~m} \cdot \mathrm{N} \cdot \mathrm{m}^{-1}\right)$ on one of our microstructured omniphobic surfaces (which is labeled as surface C) is shown in Figure 2. All droplets exhibit a similar contact angle $\left(\sim 160^{\circ}\right)$, which clearly illustrates the substrate's omniphobicity.

We fabricated a series of different microstructured substrates, the only difference being the edge-curvature and surface roughness. The resulting microstructures, labeled A-D are shown in Figure 1A-D. Their height $(h)$, interspacing $(i)$ and diameter $(d)$ were all in the same range; see Table 1 . The microstructures were placed on a square lattice with periodicity $p=d+i$ and the surface packing fraction of the structures ( ) was of the order of 5\%. The sides of the micropillars on samples A and B were smooth at the micro-scale (see the schematic representations in the insets in Figures 1A-D), while the sides of the micropillars on sample $\mathrm{C}$ were porous, giving rise to roughness at the micro-scale. On sample D the 
sockets of the pillars were smooth at the micro-scale, while their upper half was slightly porous. The edges of the pillars in sample A were very sharp (radius of curvature $<<1 \mu \mathrm{m}$ ), while the edges on sample B were more rounded (radius of curvature $\approx 1 \mu \mathrm{m}$ ). On samples $\mathrm{C}$ and $\mathrm{D}$ the top plateaus of the pillars exhibit frayed, sharp edges (radius of curvature $<<1 \mu \mathrm{m}$ ).

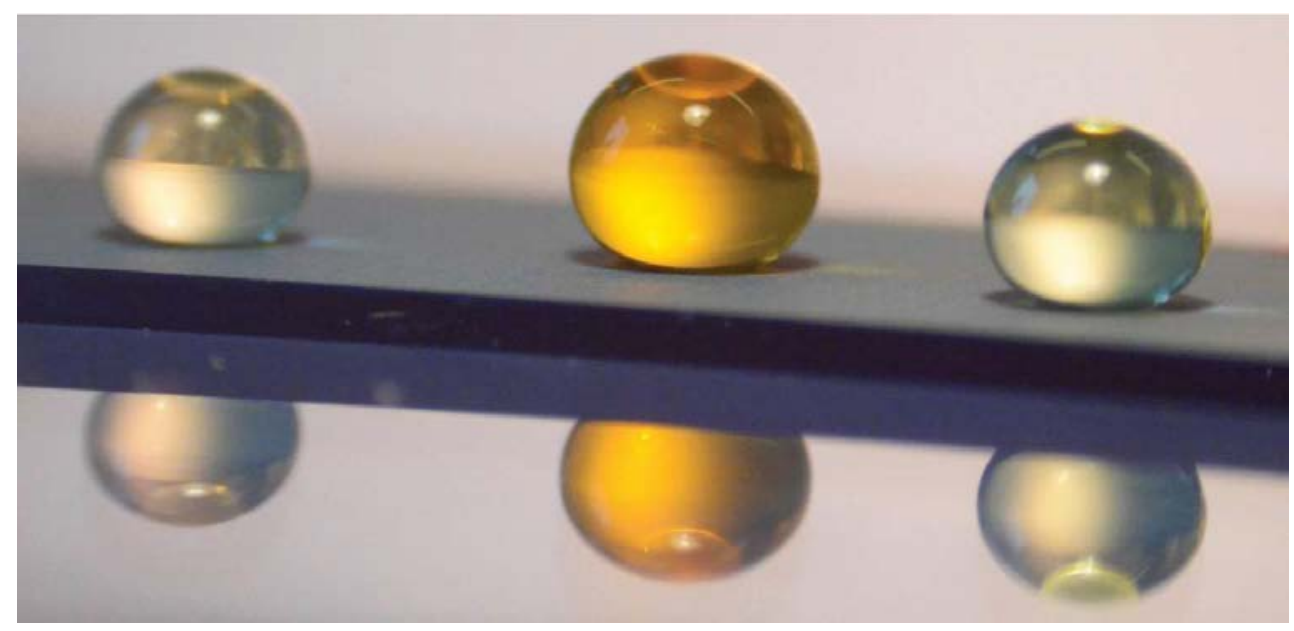

Figure 2: Demonstration of the omniphobicity of substrate C. Droplets of $\boldsymbol{n}$-octane (left, $\sigma_{l v}=$ $21.6 \mathrm{~m} . \mathrm{N} . \mathrm{m}^{-1}$ ), water (middle, $\sigma_{l v}=72.8 \mathrm{~m} . \mathrm{N} . \mathrm{m}^{-1}$ ) and olive oil (right, $\sigma_{l v}=32.5 \mathrm{~m} . \mathrm{N} . \mathrm{m}^{-1}$ ), showing contact angles of $\sim 160^{\circ}$ on this substrate.

On all samples, the contact angles for water, olive oil and $n$-octane were found to be $(150 \pm 5)^{\circ},(155 \pm 5)^{\circ}$ and $(155 \pm 5)^{\circ}$, respectively. In all cases the contact angle hysteresis was limited to less than 10 degrees. The omniphobicity of substrates A-D could be related to the re-entrant surface curvature, or, in other words, the "overHanging" microstructures. According to Tuteja et. al., ${ }^{[10]}$ such multivalued surface topography does indeed result in superhydrophobicity and superoleophobicity.

\subsubsection{Evaporation of water droplets}

The evaporation of water droplets with initial volumes ranging from 2 to $6 \mu \mathrm{L}$ was filmed from the side. Snapshots of side-view images of the evaporating droplets are shown in Figure 3. Several experiments were performed for each surface, with reproducible results. For substrates $\mathrm{A}$ and $\mathrm{B}$ a transition from the $\mathrm{CB}$ state to the $\mathrm{W}$ state is clearly visible. 


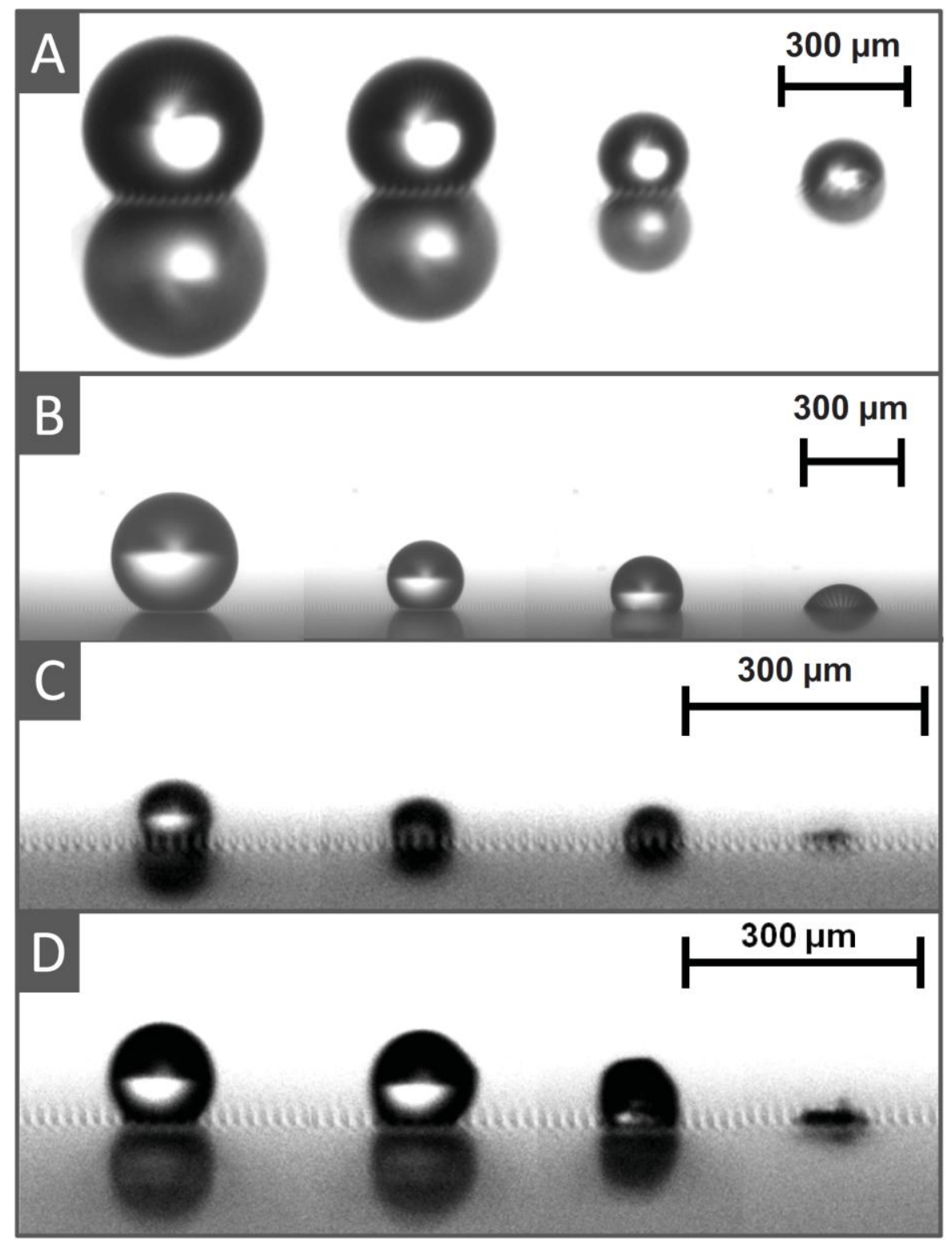

Figure 3: Snapshots of the side-views of an evaporating water droplet on the microstructured substrates A-D. The droplet on sample $A$ is in the CB state with a high contact angle in the first three snapshots, while in the last snapshot the droplet is in the $\mathrm{W}$ state. The droplet on substrate $B$ is in the CB state in the first two snapshots and has undergone the CB to W transition in the last two snapshots. On substrates $C$ and $D$ the droplet stays in the $C B$ state for its entire lifetime. Note that light and the microstructures are visible under the droplet when it is in the CB state and not when it is in the $W$ state.

On substrate A the droplet was in the CB state for the first 3 images (note the light between the droplet and the surface microstructures), while in the last snapshot, the droplet has undergone the $\mathrm{CB}$ to $\mathrm{W}$ transition: the contact angle dropped from 
$140^{\circ}$ to $\sim 80^{\circ}$ and there was no light visible under the droplet, since the water filled the space between the droplet and the surface microstructures. Such a transition was also observed between the first and the last two snapshots of the droplet on substrate B. For substrates $\mathrm{C}$ and $\mathrm{D}$ the transition was not observed in the side-view images at all.

From the side-view images we extracted the height, the base radius, and the contact angle of the droplet as a function of time. The base diameter of the evaporating droplets on the substrates A-D is shown in Figure 4 as a function of time.
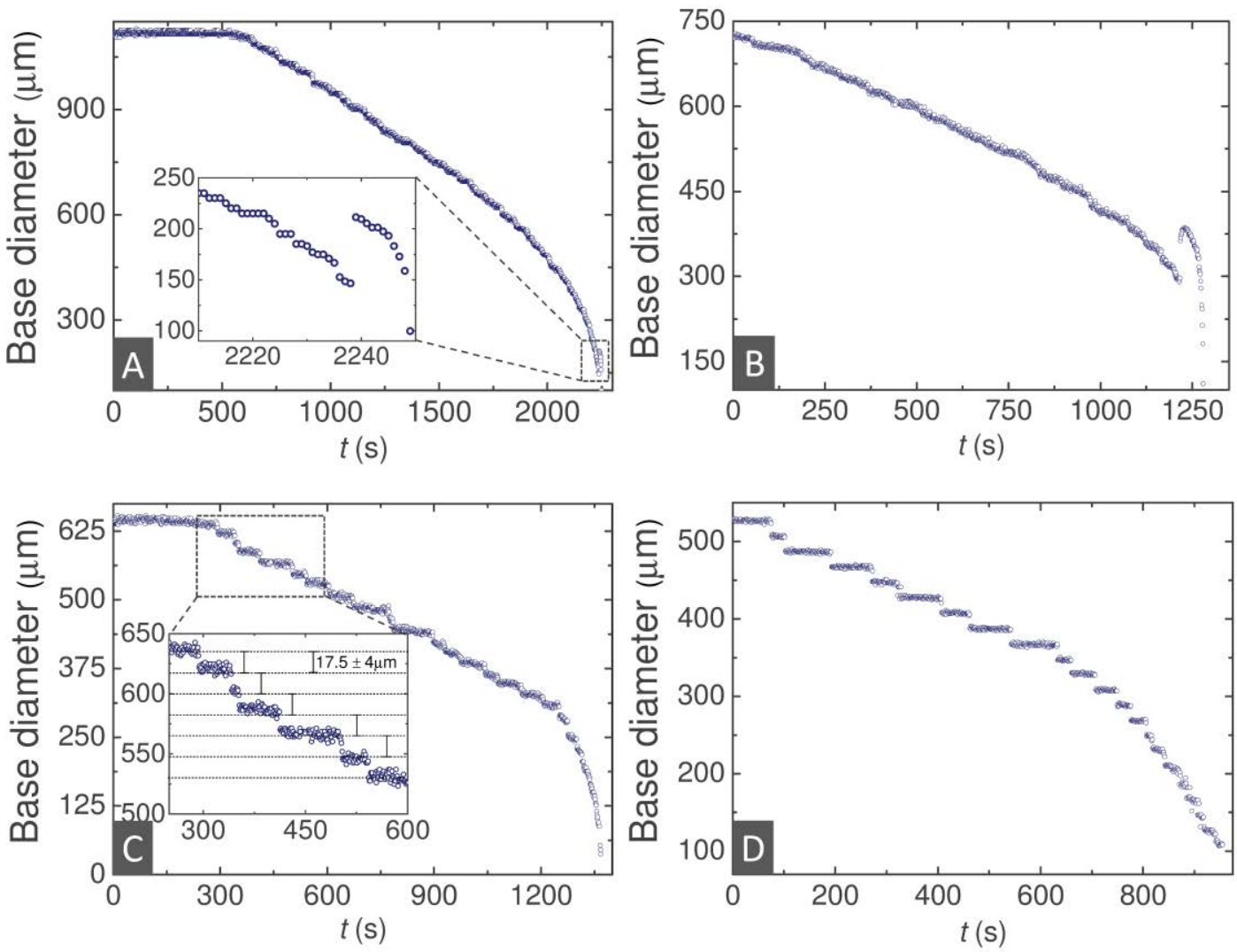

Figure 4: Base diameter of the evaporating water droplets on the substrates A-D, extracted from the side-views, as a function of time.

The evaporation time on the substrates A-D differs due to different initial volumes of the evaporating droplets. The $\mathrm{CB}$ to $\mathrm{W}$ transition on samples $\mathrm{A}$ and $\mathrm{B}$ is visible as a sudden increase in the base diameter: on sample $\mathrm{A}$ the base diameter increased from $\sim 146 \mu \mathrm{m}$ to $211 \mu \mathrm{m}$ at $t=2240 \mathrm{~s}$ (see the inset in Figure 4A) and on 
sample $B$ the base diameter increased from $\sim 300 \mu \mathrm{m}$ to $375 \mu \mathrm{m}$ at $t=1220 \mathrm{~s}$. On samples $\mathrm{C}$ and $\mathrm{D}$ such a sudden increase was not observed. Pinning of the contact-line leads to a stepwise retraction from pillar to pillar, which shows up as distinct plateaus in the plot of base diameter as a function of time in Figure 4C and D. The step size between subsequent plateaus corresponds to the interspacing between the microstructures $(=p \pm d)$.

\subsubsection{Evaporation of a colloidal solution}

The contact angle of the evaporating droplets on the substrates A-D is shown in Figure 5 as a function of time.
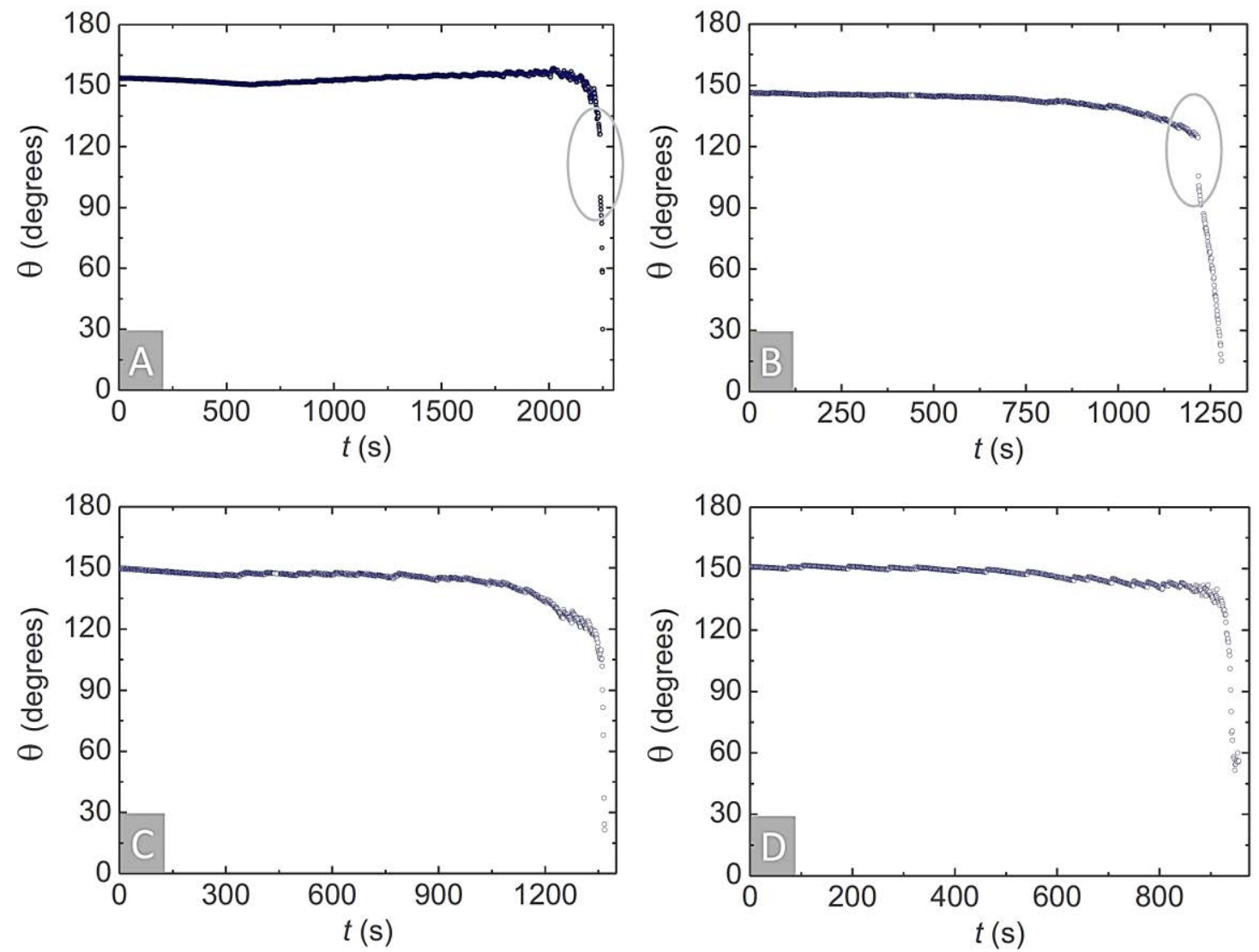

Figure 5: Contact angle, $\theta$ of the evaporating water droplets on the substrates A-D, extracted from the side-views, as a function of time. The CB to $W$ transitions on substrates $A$ and $B$ are marked by the gray ellipses.

The $\mathrm{CB}$ to $\mathrm{W}$ transition on samples $\mathrm{A}$ and $\mathrm{B}$ is noticeable as a sudden decrease in the contact angle (marked by the gray ellipses). On samples C and D such a sudden 
decrease is not observed. One could argue that the transition from the CB to the $\mathrm{W}$ state on substrate $\mathrm{C}$ and $\mathrm{D}$ may happen when the size of the droplet is beyond the resolution of our camera. To test this hypothesis we performed evaporation experiments with a colloidal suspension of spherical polystyrene particles of $1 \mu \mathrm{m}$ in diameter. After evaporation, the polystyrene particles were exclusively found on top of the microstructures (see Figure 6), which shows that the droplet was in the CB state, sitting on top of the microstructures, during its entire lifetime. In contrast, we found the polystyrene particles all over the surface in cases where a CB to W transition occurred.

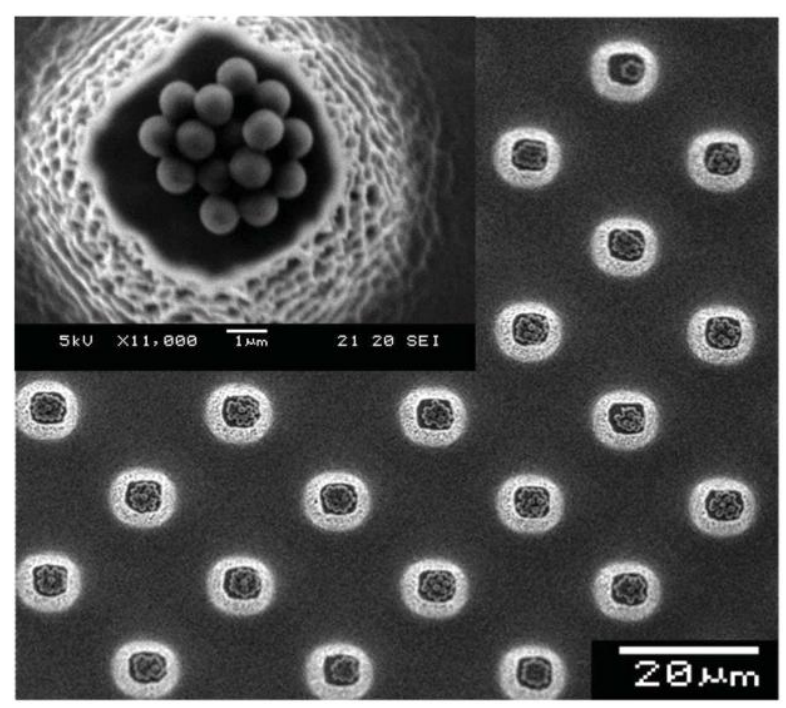

Figure 6: SEM micrographs of substrate $C$ after evaporation of a droplet containing a colloidal suspension of polystyrene particles. After evaporation of the water, the particles are exclusively found on top of the microstructures, demonstrating that the droplet remained on top of the pillars during the entire evaporation process. The inset shows a zoom-in on a single micropillar with the polystyrene particles on top of the pillar.

\subsubsection{Comparing with mechanisms from literature}

We compare our experimental data with the transition mechanisms proposed in the literature. For a Laplace-pressure driven mechanism the moment of transition will, for nanostructures of similar dimensions, be totally determined by the droplet size. Reyssat et.al. ${ }^{[32]}$ proposed that a Laplace-pressure driven transition will occur when the droplet radius $R<i^{2} / h$. For our samples (with comparable $i$ and $h$ values), this corresponds to a critical radius of $\sim 22 \pm 3 \mu \mathrm{m}$. For sample A we observed the $\mathrm{CB}$ 
to $\mathrm{W}$ transition when the base diameter was $\sim 150 \mu \mathrm{m}$. At that moment the drop had a radius of $\sim 90 \mu \mathrm{m}$, corresponding to a Laplace-pressure of $\sim 1.6 \mathrm{kPa}$. For sample B we observed the $\mathrm{CB}$ to $\mathrm{W}$ transition when the base diameter and radius were $\sim 300 \mu \mathrm{m}$ and $\sim 180 \mu \mathrm{m}$, respectively, corresponding to a Laplace-pressure of $\sim 0.8 \mathrm{kPa}$. In samples $\mathrm{C}$ and $\mathrm{D}$ the transition was never observed, even for very small droplet sizes (high Laplace-pressures). The CB to $\mathrm{W}$ transition therefore cannot be fully described by a Laplace-pressure driven mechanism.

Next we discuss the validity of a global interfacial energy argument ${ }^{[20-22,30]}$ to describe the $\mathrm{CB}$ to $\mathrm{W}$ transition. This argument is based on comparison of the interfacial energies $E_{C B}$ and $E_{W}$ for the $\mathrm{CB}$ and the W states during the evaporation process. The total interfacial energy $E_{C B}$ or $E_{W}$ is the sum of the creation energies of all interfaces. Thus,

$$
E_{W}=A_{s l} \sigma_{s l}+A_{l v} \sigma_{l v} \text { and } E_{C B}=A_{s l} \sigma_{s l}+A_{s v} \sigma_{s v}+A_{l v} \sigma_{l v}
$$

where $A$ is the interfacial area and the indices $s, l$ and $v$ indicate, as earlier, the solid, liquid and vapour phases, respectively. We used $\sigma_{l v}=72.8 \mathrm{~m} . \mathrm{N} . \mathrm{m}^{-1}$ for the droplet-air interface and $\sigma_{s v}=12$ m.N.m ${ }^{-1}$ for the FOTS-coated $\mathrm{SiO}_{2}$ surface ${ }^{[40]}$. Following the approach of Tsai et. al., ${ }^{[30]}$ the interface tension $\sigma_{s l}$ is estimated by a force balance at the contact-line using a modified Young's equation:

$$
\sigma_{s l}=\sigma_{s v}-\sigma_{l v} \cos \theta^{*}
$$

$\theta^{*}$ was determined from the side-view images of the evaporating droplets.

We illustrate this global interfacial energy argument with an example of water droplet evaporation on a substrate with straight micropillars (see the inset in Figure 7 for a SEM image of the surface). These micropillars have similar height, interspacing and diameter as structures A-D, without added curvature and roughness. The blue open circles in Figure 7 show the base diameter of the evaporating droplet as a function of time. The $\mathrm{CB}$ to $\mathrm{W}$ transition occurred at $t=2300 \mathrm{~s}$ when the base diameter had increased from $395 \mu \mathrm{m}$ to $483 \mu \mathrm{m}$. This moment is marked by the vertical line and the gray ellipses. Using the base diameter and the contact angle as extracted from the side-view images we calculated $E_{C B}$ and $E_{W}$ for each moment of the droplet's lifetime. The energy difference $E_{C B}-E_{W}$ is plotted as the closed triangles 
in Figure 7. From the beginning of the evaporation process until $t=2300 \mathrm{~s}$ the energy difference $E_{C B}-E_{W}$ is negative, since the $\mathrm{CB}$ state has a lower energy than the $\mathrm{W}$ state and, hence, the droplet is in the CB state. After $t=2300 \mathrm{~s}$ the $\mathrm{W}$ state has the lowest energy. The $\mathrm{CB}$ to $\mathrm{W}$ transition is occurring when $E_{C B}=E_{W}$, i.e. at $t=2300 \mathrm{~s}$, which is exactly the moment when the $\mathrm{CB}$ to $\mathrm{W}$ transition is observed in the side-view images of the droplet.

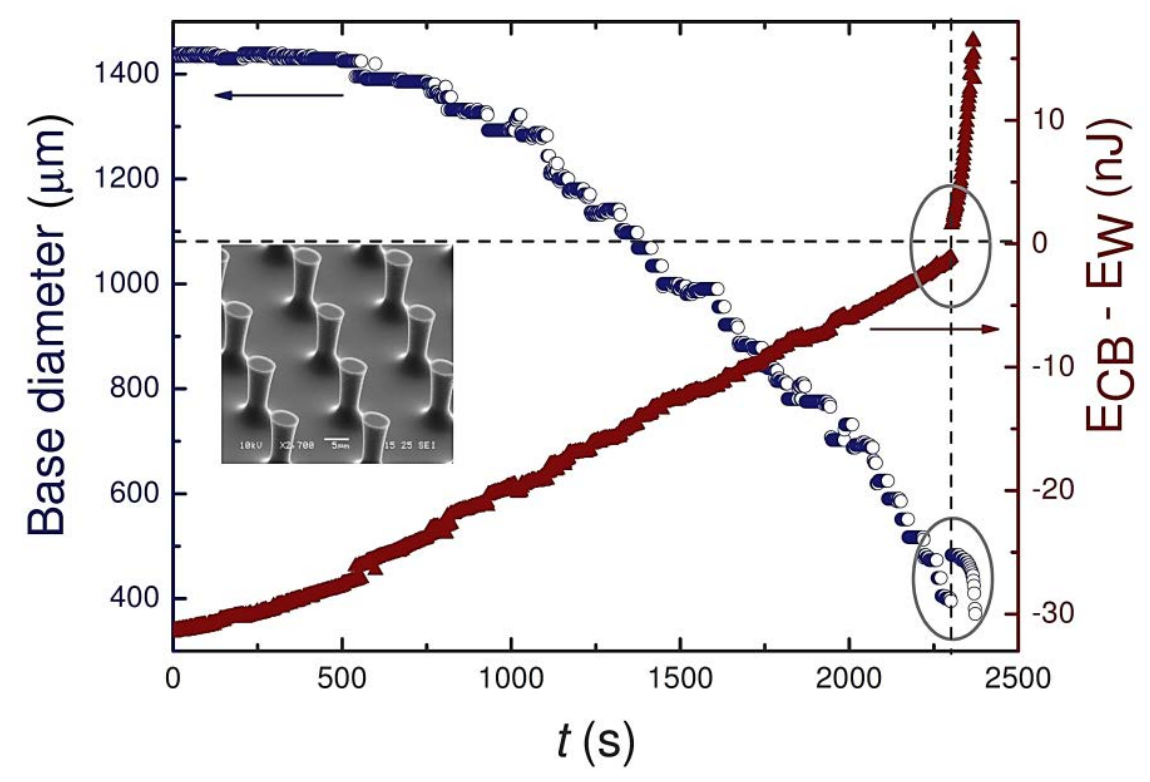

Figure 7: Illustration of the global interfacial energy argument on a substrate containing micropillars with straight interfaces and sharp edges (see the inset for a SEM micrograph). The open circles (referring to the left-hand ordinate) represent the base diameter of the evaporating water droplet as a function of time. The closed triangles (referring to the right-hand ordinate) show the calculated interfacial energy difference $E_{C B}-E_{W}$. The horizontal line is positioned at $E_{C B}-E_{W}=0$ and the vertical line marks the time when the $C B$ to $W$ transition occurs, exactly when $E_{C B}-E_{W}=0$.

Figure 8 displays the calculated energy difference $E_{C B}-E_{W}$ for the evaporating droplets on the substrates A-D as a function of time. For the droplet on substrate A the energy difference $E_{C B}-E_{W}$ is positive until $t=1250 \mathrm{~s}$, indicating that in this period the $\mathrm{W}$ state has the lowest energy. This observation is in agreement with the findings of Kwon et.al. ${ }^{[23]}$ for larger droplets. From $t=1250 \mathrm{~s}$ until $t=2220 \mathrm{~s}$ the energy difference $E_{C B}-E_{W}$ is negative, while from $t=2220 \mathrm{~s}$, the CB state becomes 
lower in energy again. Note that the CB to $\mathrm{W}$ transition occurred at $t=2238 \mathrm{~s}$ on substrate A. The calculated lowest energy state in the initial stages of the droplet evaporation on substrates B-D is the CB state. During the evaporation process of each droplet, the calculated energy difference $E_{C B}-E_{W}$ equals zero at some moment. This moment is, however, not in agreement with the experimentally observed $\mathrm{CB}$ to $\mathrm{W}$ transition on substrates $\mathrm{A}$ and $\mathrm{B}$ and with the absence of a transition on substrates $\mathrm{C}$ and D. Note that for substrates $C$ and $D$ the exact surface roughness of the walls of the micropillars is unknown. Calculations were therefore performed at different roughness values, namely for $r=1, r=2$, and $r=4$, with $r$ defined as in Equation (1). In all cases the calculated energy difference $E_{C B}-E_{W}$ goes through zero during the evaporation of the droplet, but no $C B$ to $W$ transition is experimentally observed.
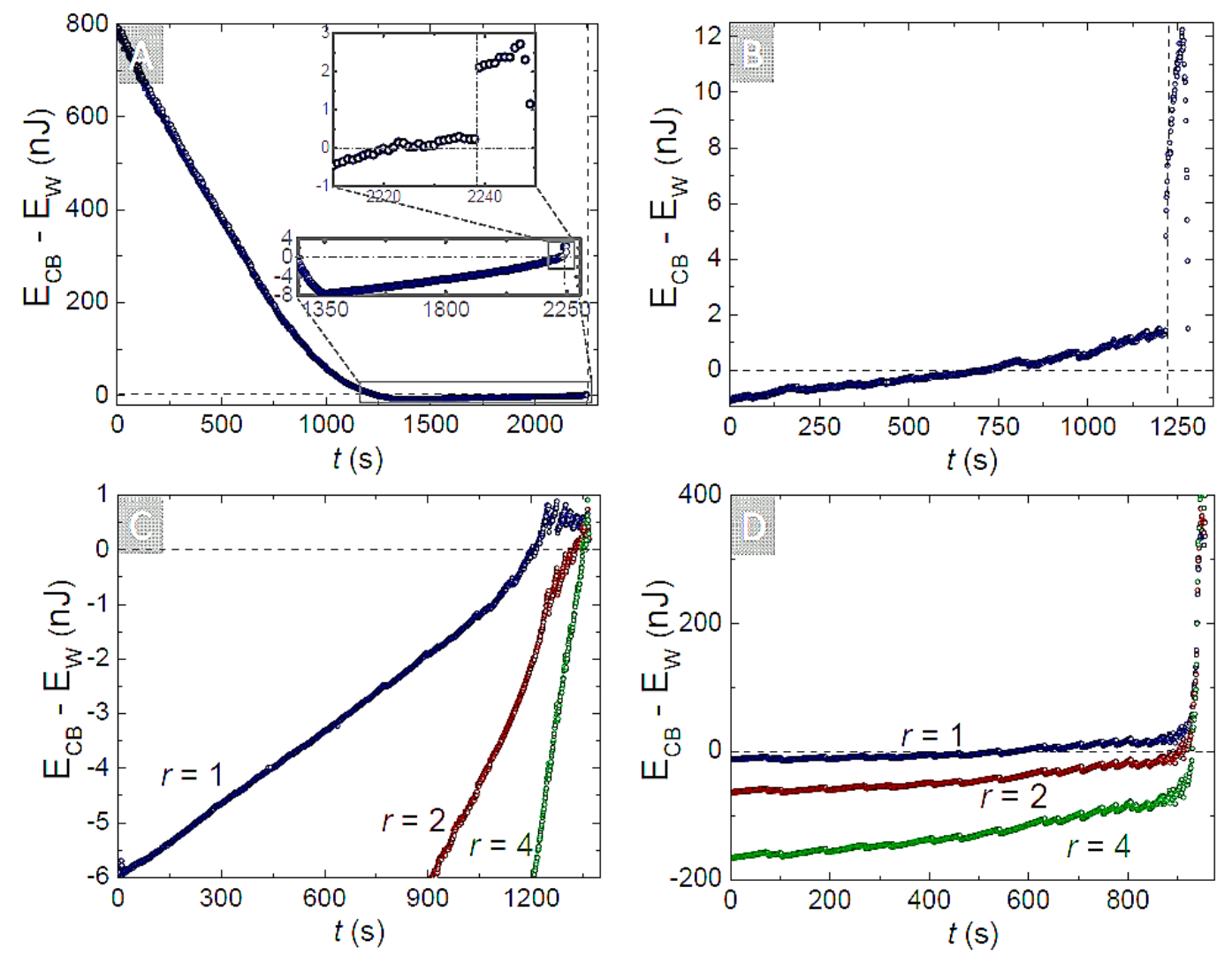

Figure 8: Calculated interfacial energy difference $E_{C B}-E_{W}$ for the evaporating water droplets on the substrates A-D as a function of time. The vertical lines in graphs $A$ and $B$ mark the time when the CB to $\mathrm{W}$ transitions occurs. For substrates $\mathrm{C}$ and $\mathrm{D}$ the exact surface roughness on the sides of the pillars is unknown. The energy difference $E_{C B}-E_{W}$ is calculated for three roughness values, namely, $r=1$ (blue), $r=2$ (brown) and $r=4$ (green). The dotted horizontal lines are positioned at $E_{C B}-E_{W}=0$. 


\subsubsection{The global energy argument crisis}

The presented global interfacial energy argument obviously fails to predict the $\mathrm{CB}$ to $\mathrm{W}$ transitions on our substrates: For the droplet on substrate $\mathrm{A}$ it predicts the $\mathrm{CB}$ to $\mathrm{W}$ transition at $t=2220 \mathrm{~s}$, while in the earlier stages of the evaporation process (from $t=0$ to $t=1250 \mathrm{~s}$ ), the $\mathrm{W}$ state was calculated to be the lowest in energy. Experimentally it was observed that the droplet was in the CB state till $t=2238 \mathrm{~s}$. For the droplet on substrate B the global interfacial energy argument predicts a transition at $t=650 \mathrm{~s}$, while the observed transition occurred at $t=1220 \mathrm{~s}$. For the droplets on substrates $\mathrm{C}$ and $\mathrm{D}$, all the calculations predict a $\mathrm{CB}$ to $\mathrm{W}$ transition, while such a transition was never observed on these substrates.

Why is the global interfacial energy argument, as presented above, insufficient to explain the experimental observations for our substrates A-D? In the above described interfacial energy argument only the interfacial energies are taken into account. The positions of the (global) energy minima may be correctly determined from such a calculation. Possible barriers to the $\mathrm{CB}$ to $\mathrm{W}$ transition, are, however, not taken into account. When the $\mathrm{CB}$ to $\mathrm{W}$ transition is kinetically hindered, barriers should be taken into account and the above described interfacial energy argument fails to describe the transition. The possible existence of an energy barrier between the $\mathrm{CB}$ and the $\mathrm{W}$ state is, among others, described by Patankar ${ }^{[39,41]}$ and Gao and McCarthy ${ }^{[42]}$. Koishi et. al. ${ }^{[38]}$ calculated a barrier on the basis of statisticalmechanics. Savoy and Escobedo ${ }^{[43]}$ reported detailed information about the transition kinetics and mechanism for small droplets on the basis of molecular dynamic (MD) simulations. Nosonovsky ${ }^{[44]}$ reported the existence of energy barriers for surfaces with a re-entrant surface curvature. The variation in the sharpness of the edges and the surface roughness of the micropillars on substrate A-D results in different energy barriers which must be overcome by the evaporating droplet in order to reach the energy minimum in the $\mathrm{W}$ state. The development of a model to predict the $\mathrm{CB}$ to $\mathrm{W}$ transition that includes the possible energy barrier between the $\mathrm{CB}$ and the $\mathrm{W}$ states is needed. MD simulations seem promising to fill this gap ${ }^{[43]}$. Our findings emphasize that to design omniphobic substrates, not only the geometrical shape and arrangement of the microstructures but also the (nano)roughness and edge-curvature should be taken into account. 


\subsection{Conclusions}

In summary, we have produced a series of substrates which display high equilibrium contact angles $\left(\theta>150^{\circ}\right)$ for water, $n$-octane and olive oil. We have studied the evaporation-driven wetting transition of water on these substrates. It was found that $\mathrm{CB}$ to $\mathrm{W}$ transition can be either substantially delayed or totally avoided by changing the edge curvature of the microstructures and addition of roughness to the micropillar walls. We verified that neither a Laplace-pressure driven mechanism nor a global interfacial energy argument describes the CB to $\mathrm{W}$ transition on these surfaces correctly, since they are not able to estimate the energy barrier that separates the CB and the $\mathrm{W}$ states. The added roughness and edge-curvature were concluded to be the physical origin of this energy barrier. For pillars with sharp edges and straight interfaces, the global interfacial energy argument can be successfully applied to predict the $\mathrm{CB}$ to $\mathrm{W}$ transition, indicating the absence of an energy barrier on this substrate. Our results convincingly show that even though the added edge-curvature and roughness did not significantly change the equilibrium contact angle, they greatly enhanced the dynamical robustness of our omniphobic substrates.

\subsection{References}

[01] D. Quéré, Annu. Rev. Mater. Res., 2008, 38, 71.

[02] W. Barthlott and C. Neinhuis, Planta, 1997, 202, 1

[03] M. Liu, Y. Zheng, J. Zhai, and L. Jiang, Acc. Chem. Res., 2010, 43, 368.

[04] J. W. M. Bush and D. L. Hu, Annu. Rev. Fluid Mech., 2006, 38, 339.

[05] R. Blossey, Nat. Mater., 2003, 2, 301.

[06] J. Rothstein, Annu. Rev. Fluid Mech., 2010, 42, 89.

[07] G. McHale, M. Newton, and N. Shirtcliffe, Soft Matter, 2009, 6, 714.

[08] A. J. Meuler, G. H. McKinley, and R. E. Cohen, ACS Nano, 2010, 4, 7048.

[09] K. K. Varanasi, M. Hsu, N. Bhate, W. Yang, and T. Deng, Appl. Phys. Lett., 2009, 95, 094101. 
[10] A. Tuteja, W. Choi, M. Ma, J. M. Mabry, S. A. Mazella, G. C. Rutledge, G. H. McKinley, and R. E. Cohen, Science, 2007, 318, 1618.

[11] A. Susarrey-Arce, A. G. Marin, S. Schlautmann, L. Lefferts, A. van Houselt, and J. G. E. Gardeniers, ACS Appl. Mater. Interfaces, 2012, submitted.

[12] W. Wu, X. Wang, D. Wang, M. Chen, F. Zhou, W. Liu, and Q. Xue, Chem. Commun., 2009, 1043.

[13] L. Cao, T. P. Price, M. Weiss, and D. Gao, Langmuir, 2008, 24, 1640.

[14] H. Zhao, K.-Y. Law, and V. Sambhy, Langmuir, 2011, 27, 5927.

[15] R. Dufour, P. Brunet, M. Harnois, R. Boukherroub, V. Thomy, and V. Senez, Small, 2012, 8, 1229.

[16] R. N. Wenzel, Ind. Eng. Chem., 1936, 28, 988.

[17] A. D. B. Cassie and S. Baxter, Trans. Faraday Soc., 1944, 40, 546.

[18] D. Bonn, J. Eggers, J. Indekeu, J. Meunier, and E. Rolley, Rev. Mod. Phys., 2009, 81, 739 .

[19] H. Y. Erbil and C. E. Cansoy, Langmuir, 2009, 25, 14135.

[20] M. Sbragaglia, A. M. Peters, C. Pirat, B. M. Borkent, R. G. H. Lammertink, M. Wessling, and D. Lohse, Phys. Rev. Lett., 2007, 99, 156001.

[21] C. Pirat, M. Sbragaglia, A. M. Peters, B. M. Borkent, R. G. H. Lammertink, M. Wessling, and D. Lohse, Europhys. Lett., 2008, 81, 66002.

[22] A. M. Peters, C. Pirat, M. Sbragaglia, B. M. Borkent, M. Wessling, D. Lohse, and R. G. H. Lammertink, Eur. Phys. J. E: Soft Matter Biol. Phys., 2009, 29, 391.

[23] H.-M. Kwon, A. T. Paxson, K. K. Varanasi, and A. Patankar, Phys. Rev. Lett., 2011, 106, 036102.

[24] G. Manukyan, J.M. Oh, D. van den Ende, R. G. H. Lammertink, and F. Mugele, Phys. Rev. Lett., 2011, 106, 014501.

[25] D. Bartolo, F. Bouamrirene, E. Verneuil, A. Buguin, P. Silberzan, and S. Moulinet, Europhys. Lett., 2006, 74, 299.

[26] P. Tsai, S. Pacheco, C. Pirat, L. Lefferts, and D. Lohse, Langmuir, 2009, 25, 12293.

[27] E. Bormashenko, R. Pogreb, G. Whyman, Y. Bormashenko, and M. Erlich, Appl. Phys. Lett., 2007, 90, 201917.

[28] Y. C. Jung and B. Bhushan, Langmuir, 2009, 25, 9208. 
[29] C. Journet, S. Moulinet, C. Ybert, S. T. Purcell, and L. Bocquet, Europhys. Lett., 2005, 71, 104.

[30] P. Tsai, R. G. H. Lammertink, M. Wessling, and D. Lohse, Phys. Rev. Lett., 2010, 104, 116102.

[31] G. McHale, S. Aqil, N. J. Shirtcliffe, M. I. Newton, and H. Y. Erbil, Langmuir, 2005, 21, 11053.

[32] M. Reyssat, J. M. Yeomans and D. Quéré, Europhys. Lett., 2008, 81, 26006.

[33] O. Bliznyuk, V. Veligura, E. S. Kooij, H. J. W. Zandvliet and B. Poelsema, Phys. Rev. E: Stat., Nonlinear, Soft Matter Phys., 2011, 83, 041607.

[34] H. Gelderblom, A. G. Marin, H. Nair, A. van Houselt, L. Lefferts, J. H. Snoeijer, and D. Lohse, Phys. Rev. E: Stat., Nonlinear, Soft Matter Phys., 2011, 83, 026306.

[35] S. Moulinet and D. Bartolo, Eur. Phys. J. E, 2007, 24, 251.

[36] H. Kusumaatmaja, M. L. Blow, A. Dupuis, and J. M. Yeomans, Europhys. Lett., 2008, 81, 36003.

[37] L. Barbieri, E. Wagner, and P. Hoffmann, Langmuir, 2007, 23, 1723.

[38] T. Koishi, K. Yasuoka, S. Fujikawa, T. Ebisuzak, and X. C. Zeng, Proc. Natl. Acad. Sci., 2009, 106, 8435.

[39] N. A. Patankar, Langmuir, 2004, 20, 7097.

[40] A. Amassian, V. A. Pozdin, T. V. Desai, S. Hong, A. R. Woll, J. D. Ferguson, J. D. Brock, G. G. Malliaras, and J. R. Engstrom, J. Mater. Chem., 2009, 19, 5580.

[41] B. He, N. A. Patankar, and J. Lee, Langmuir, 2003, 19, 4999.

[42] L. Gao and T. J. McCarthy, Langmuir, 2007, 23, 3762.

[43] E. S. Savoy and F. A. Escobedo, Langmuir, 2012, 28, 3412.

[44] M. Nosonovsky, Langmuir, 2003, 23, 3157. 


\section{Chapter 3}

\section{Influence of thin film nickel pretreatment on catalytic thermal chemical vapor deposition of carbon nanofibers ${ }^{1}$,}

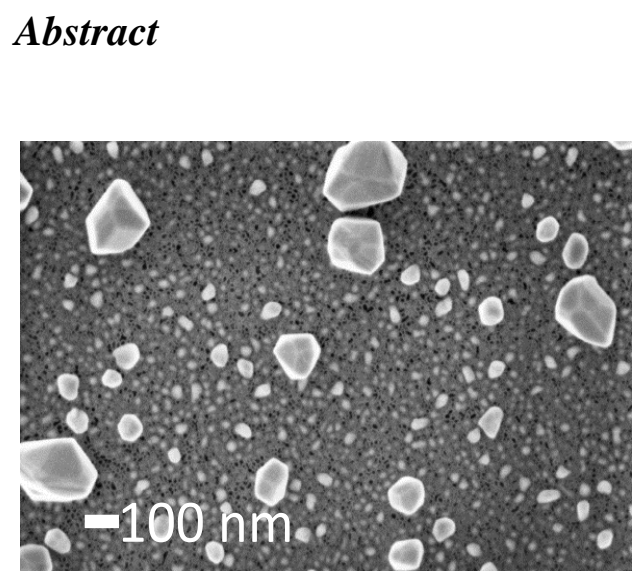

Nickel (Ni) and other metal nanoparticles are known to be active as catalysts in the synthesis of carbon nanofibers. In this chapter we investigate how dewetting and break-up of nickel thin films depend on film thickness, film substrate interaction and pretreatment conditions. This is evaluated for films evaporated on oxidized silicon and fused silica substrates with or without tantalum (Ta) coating, which were subsequently exposed to different pretreatment atmospheres (vacuum, nitrogen, air and hydrogen; $1 \mathrm{~h}, 650{ }^{\circ} \mathrm{C}$ ). Atomic force microscopy, scanning electron microscopy and energy dispersive $X$-ray analysis were used to characterize the films. Pretreated Ni films were subjected to a thermal catalytic chemical vapor deposition procedure with brief ethylene exposures $\left(0.5-3 \mathrm{~min}, 635{ }^{\circ} \mathrm{C}\right)$. It was found that only on the spherical nanoparticles originating from a hydrogen pretreatment of a Ni film with Ta adhesion layer, homogeneously distributed, randomly-oriented, wellattached, and semi-crystalline carbon nanofibers be synthesized.

\footnotetext{
${ }^{1}$ This chapter is published as "Influence of thin film nickel pretreatment on catalytic thermal chemical vapor deposition on carbon nanofibers" by R. M. Tiggelaar, D. B. Thakur, H. Nair, L. Lefferts. K. Seshan, J. G. E. Gardeniers, Thin solid Films, 2013, 534, 341.

*Catalyst substrate preparations with R.M.Tiggelaar, Experiments on fused Si with D.B. Thakur.
} 


\subsection{Introduction}

Due to their exceptional mechanical, physical, electrical, and chemical properties, ${ }^{[1-5]}$ carbon nanofibers (CNFs) and carbon nanotubes (CNTs) are used in a wide variety of applications, ranging from composite reinforcing, ${ }^{[6]}$ chemical sensing ${ }^{[7,8]}$ and hydrogen storage ${ }^{[9]}$ to superhydrophobic surfaces, ${ }^{[10]}$ field emitters, ${ }^{[11]}$ electrodes in fuel cells and plasma microreactors, ${ }^{[12,13]}$ and catalyst supports in microreactors. $^{[14-17]}$

A crucial role in the growth of CNFs and CNTs by thermal catalytic chemical vapor deposition (TC-CVD) is played by the nanoparticles composed of a transition metal like cobalt $(\mathrm{Co})$, iron (Fe), and nickel (Ni), or their alloys, which are used to catalyze the synthesis of the nanostructures from hydrocarbon gases like methane $\left(\mathrm{CH}_{4}\right)$, acetylene $\left(\mathrm{C}_{2} \mathrm{H}_{2}\right)$, ethylene $\left(\mathrm{C}_{2} \mathrm{H}_{4}\right)$, ethane $\left(\mathrm{C}_{2} \mathrm{H}_{6}\right)$, carbon monoxide $(\mathrm{CO})$ or synthesis gas (mixture of $\mathrm{CO}$ and hydrogen $\left(\mathrm{H}_{2}\right)$ ), at temperatures between $400{ }^{\circ} \mathrm{C}$ and $1000{ }^{\circ} \mathrm{C}$. CNFs are claimed to be formed by a solution-diffusion-precipitation process that generates graphitic carbon. ${ }^{[18]}$ Hydrocarbon molecules decompose at the surface of the nanoparticle, and carbon atoms dissolve into the metal forming a solid solution, from which upon super-saturation graphite layers nucleate and grow at the nanoparticle surface by diffusion-driven precipitation. ${ }^{[19-21]}$ This preferentially occurs at stressed locations, like dislocations and grain boundaries, with the consequence that polycrystalline metal nanoparticles offer many sites for precipitation of carbon and nanostructure growth. ${ }^{[19]}$ The effect of nanoparticle size and synthesis temperature on CNF nucleation and growth can be understood on the basis of the temperature dependence of the solubility $(S)$ and diffusivity $(D)$ of carbon, which both increase with temperature. Thus, at a fixed temperature nucleation and growth rate are higher on smaller particles, due to the effect that the diffusion flux in the particles is proportional to their surface-to-volume ratio. ${ }^{[22]}$ For a given nanoparticle size, CNF growth rate increases with temperature, ${ }^{[23]}$ so that on large nanoparticles at high synthesis temperatures CNFs will nucleate and grow to larger diameters, ${ }^{[19,20,22,23]}$ while at low synthesis temperatures no growth occurs. In the latter case particles may become catalytically inactive due to coverage with carbon layers. ${ }^{[19,20]}$ Above a certain temperature a decrease in yield occurs, which is due to catalyst deactivation as 
a result of a disturbed balance between graphite formation kinetics and amorphous carbon deposition $^{[22]}$ or due to loss of catalyst by e.g. evaporation. ${ }^{[24,25]}$ Addition of ammonia $\left(\mathrm{NH}_{3}\right)$ or $\mathrm{H}_{2}$ to the hydrocarbon source maintains the nanoparticles catalytically active over a wider temperature range by reducing coverage by amorphous carbon. ${ }^{[22,26-29]}$

From the above it is clear that a well-defined catalyst nanoparticle size is essential for control of CNF growth rate and morphology. Although it is possible to start with pre-patterned catalyst islands (e.g. defined by photolithography), usually dispersed metal nanoparticles are obtained by deposition of a continuous metal film followed by a sintering process which leads to substrate dewetting and break-up of the film because of interfacial energy minimization. The resulting very small particles agglomerate into larger crystals at higher thermal budget, ${ }^{[24,27,28,30,31]}$ for which process three principal mechanisms have been identified: i) crystallite migration, ii) atomic migration via the surface, iii) atomic migration via the vapor. ${ }^{[32]}$ Determining factors are surface and bulk atom mobility, and residual stress (e.g. resulting from thermal expansion mismatch between substrate and film). For fixed temperature and atmosphere, pretreatment of thinner films yields smaller particles with a sharp size distribution and a high surface density (in this work defined as the amount of metallic particles per unit area). Pretreatment atmosphere can have an effect on particle formation, especially for metals which strongly interact with oxides or are easily oxidized themselves, which due to a lower surface mobility give low density, large nanoparticles. This can be by-passed by a reducing atmosphere, ${ }^{[19,22,23,26-28,33-35]}$ e.g., for Fe evaporated on oxidized silicon, smaller islands are formed in $\mathrm{NH}_{3}$ and $\mathrm{H}_{2}$ with respect to vacuum, air or argon (Ar). ${ }^{[28]}$

Ni films do not adhere well to materials like oxidized silicon and fused silica, and therefore an intermediate layer to improve the adhesion is often applied. For applications at temperatures above $500{ }^{\circ} \mathrm{C}$, a $10-20 \mathrm{~nm}$ thick layer of Ti-W or Ta is optimal. ${ }^{[14]}$ Although silicide formation can be prevented with a thin oxide layer $\left(>4 \mathrm{~nm}^{[24]}\right)$, it can also be used for anchoring of metallic nanoparticles on which basetype grown carbon nanostructures can be synthesized, provided enough metal of the nanoparticles remains to catalyze CNF synthesis. ${ }^{[33]}$ An implication of the use of an 
adhesion layer is that dewetting and nanoparticle formation will become different. $^{[22,35,36]}$

In this contribution we present new experimental data on dewetting of $\mathrm{Ni}$ thin films which were evaporated on oxidized silicon and fused silica substrates with or without Ta adhesion layer, pretreated in different atmospheres and subsequently briefly exposed to a TCCVD synthesis protocol $\left(0.5-3 \mathrm{~min}, 635^{\circ} \mathrm{C}\right)$ in order to obtain CNFs. The effect of thermal pretreatment conditions (vacuum, nitrogen, air and hydrogen; $1 \mathrm{~h}, 650^{\circ} \mathrm{C}$ ) on the initial properties of the metal film, its interaction with the substrate, and the generated catalyst nanoparticles is systematically studied.

\subsection{Experimental Methods}

\subsubsection{Preparation of nickel thin films}

$\mathrm{Ni}$ thin films were deposited on $\mathrm{Si}\{100\}$ substrates (p-type, resistivity 5-10 $\Omega . c \mathrm{~m}, 100 \mathrm{~mm}$ diameter, thickness $525 \mu \mathrm{m}$, single side polished; Okmetic, Finland) with a ca. $250 \mathrm{~nm} \mathrm{SiO}_{2}$ layer prepared by steam oxidation, and fused silica substrates (UV Grade 7980F, diameter $100 \mathrm{~mm}$, thickness $500 \mu \mathrm{m}$; Corning, USA). The substrates were ultrasonically cleaned in de-mineralized water for $10 \mathrm{~min}$, followed by immersion in fuming 100\% nitric acid (Selectipur 100453, BASF) for $10 \mathrm{~min}$, and boiling 69\% nitric acid (VLSI 116445, BASF) for $15 \mathrm{~min}$, rinsing in de-mineralized water, and dry spinning. In order to avoid damaging of deposited thin films during dicing, prior to metal deposition squares of $8 \mathrm{~mm} \times 8 \mathrm{~mm}$ were defined on the substrates via spin-coated photoresist (Olin 907-12). Via evaporation at pressures below $10^{-5} \mathrm{~Pa}$ (Balzers BAK600 electron-gun system), several different configurations of metal thin films were deposited, viz. $10 \mathrm{~nm} \mathrm{Ni}$ on oxidized silicon, $25 \mathrm{~nm} \mathrm{Ni}$ on oxidized silicon, and $25 \mathrm{~nm} \mathrm{Ni}+10 \mathrm{~nm}$ Ta on oxidized silicon and on fused silica. The purity of the Ni target material was $99.99 \%$, and at least $99.95 \%$ for the adhesion metal Ta; evaporation rates (controlled by an in-situ thickness monitor) were 1-5 $\AA . \mathrm{s}^{-1}$ for Ta and 10-15 $\AA . \mathrm{s}^{-1}$ for Ni. After metal deposition, an ultrasonic liftoff step in acetone (VLSI, 100038, BASF) was carried out for over $20 \mathrm{~min}$, followed by immersion in isopropanol for $10 \mathrm{~min}$ (VLSI 107038, BASF), rinsing in de-mineralized water for $10 \mathrm{~min}$, and dry spinning. Finally, the Ni-coated substrates 
were diced into samples of $1 \mathrm{~cm} \times 1 \mathrm{~cm}$ (Disco DAD-321 dicing machine).

\subsubsection{Pretreatment for the formation of nanoparticles}

The samples were cleaned in acetone for 5 min (Branson 200 ultrasonic cleaner) to remove organic contaminants, followed by rinsing in de-ionized water and drying with pressurized technical air. The samples were annealed in a tubular quartz reactor (heated externally by a horizontal three-zone furnace, Elicra Electrowarmte B.V.) with a ramp of $5 \mathrm{~K} \cdot \mathrm{min}^{-1}$ from room temperature to $650{ }^{\circ} \mathrm{C}$, kept at this temperature for $1 \mathrm{~h}$, and cooled down to room temperature. Four different annealing atmospheres were used: vacuum (down to $700 \mathrm{~Pa}$ ), nitrogen $\left(\mathrm{N}_{2}, 99.95 \%\right.$ PRAXAIR, ambient pressure, flow rate $50 \mathrm{ml} \cdot \mathrm{min}^{-1}$ ), air (technical air, ambient pressure, flow rate $\left.50 \mathrm{ml} \cdot \mathrm{min}^{-1}\right)$, and hydrogen (99.999\%, INDUGAS; 20 vol. $\% \mathrm{H}_{2}$ in $\mathrm{N}_{2}$, total flow rate $\left.50 \mathrm{ml} \cdot \mathrm{min}^{-1}\right)$. The reducing pretreatment in hydrogen atmosphere was also carried out at $500{ }^{\circ} \mathrm{C}(2 \mathrm{~h}), 600{ }^{\circ} \mathrm{C}(1 \mathrm{~h})$, and $700{ }^{\circ} \mathrm{C}(1 \mathrm{~h})$.

\subsubsection{Synthesis of CNFs}

Synthesis was performed in a quartz reactor heated by a horizontal three-zone furnace (Elicra Electrowarmte B.V.), after ramping up in $\mathrm{N}_{2}$ from room temperature at a rate of $5 \mathrm{~K} \cdot \mathrm{min}^{-1}$ to a temperature of $635^{\circ} \mathrm{C}$, where $\mathrm{CNF}$ formation was performed in 25 vol.\% ethylene in $\mathrm{N}_{2}$ (total flow rate $100 \mathrm{ml} . \mathrm{min}^{-1}$ ). After a reaction time of $30 \mathrm{~s}$, 1 min or $3 \mathrm{~min}$, the samples were cooled down in $\mathrm{N}_{2}$ to room temperature. Samples exposed to different pretreatment atmospheres were subjected to identical CNF growth conditions in the same run.

\subsubsection{Characterization of nanoparticles and CNFs}

As-deposited and pretreated $\mathrm{Ni}$ thin films were characterized with atomic force microscopy (AFM; Nanoscope IV, Veeco Instruments, tapping mode, tip radius $30 \pm 7.5 \mu \mathrm{m})$ to obtain information on height of nanoparticles. High-resolution scanning electron microscope top-view imaging (HR-SEM; LEO 1550) in combination with ImageJ software was used to evaluate size, distribution and density of nanoparticles. For each combination of sample composition and pretreatment condition, 3 to 5 images were recorded at $50 \times$ magnification (acceleration voltages: $4 \mathrm{kV}$ for silicon, $1 \mathrm{kV}$ for fused silica). Of each SEM-image, the centre-area of 
$5 \mu \mathrm{m} \times 7 \mu \mathrm{m}$ was considered: this area was converted into gray-scale values (8-bit), followed by binarization, thresholding, identification of the edges of particles and discretization (all by means of a standard "particle counting and analysis" toolbox in ImageJ) in order to analyse the metallic particle size, distribution and density. Origin was used to average data gathered from various SEM-images recorded on similarly pretreated and composed samples. Energy dispersive X-ray analysis (EDX; Thermo Noran Vantage system, accelerating voltage $15 \mathrm{keV}$, lifetime $50 \mathrm{~s}$ ) was applied to determine the composition of the particles (area scans of $250 \mathrm{~nm} \times 250 \mathrm{~nm}$ and $500 \mathrm{~nm} \times 500 \mathrm{~nm}$ on at least 3 randomly selected locations at $100 \times$ magnification). After the CNF synthesis process, HR-SEM imaging and room temperature Raman spectroscopy (Senterra Raman microscope spectrometer, Bruker, excitation wavelength $532 \mathrm{~nm}$ ) were used to analyze the carbon-coating/CNF coating and its crystallinity.

\subsection{Results and Discussions}

\subsubsection{Pretreatment and Ni nanoparticle formation}

Dewetting of as-deposited continuous metallic thin films involves distinct stages, i.e. hole nucleation, growth of holes, intersection of holes, rivulet retraction and formation of metallic particles. ${ }^{[37-39]}$ The stage of the dewetting process which is accomplished depends, amongst other things, on the pretreatment settings (temperature, time, environment) to which a sample is exposed. The number of metallic particles (and their size and shape) that are eventually formed is influenced by the number of film instabilities, which on its turn is affected by the composition and thickness of the thin film, deposition conditions and type of substrate. In Figure 1, SEM-images are shown for four Ni thin film configurations annealed for $1 \mathrm{~h}$ at $650{ }^{\circ} \mathrm{C}$ in different atmospheres. All investigated films reveal a stage of the dewetting process: up to the formation and/or intersection of holes in case of a pretreatment in vacuum and air, whereas particles are visible for pretreatments in $\mathrm{N}_{2}$ and $\mathrm{H}_{2}$.

Clearly, the annealing atmosphere, the thickness of the $\mathrm{Ni}$ film and the absence/presence of a Ta adhesion layer heavily influence the dewetting process. No 
morphological differences between films on oxidized silicon and fused silica are observed (gray-scale differences in the respective images are due to different accelerating voltage settings during SEM imaging, to avoid electrical charging).
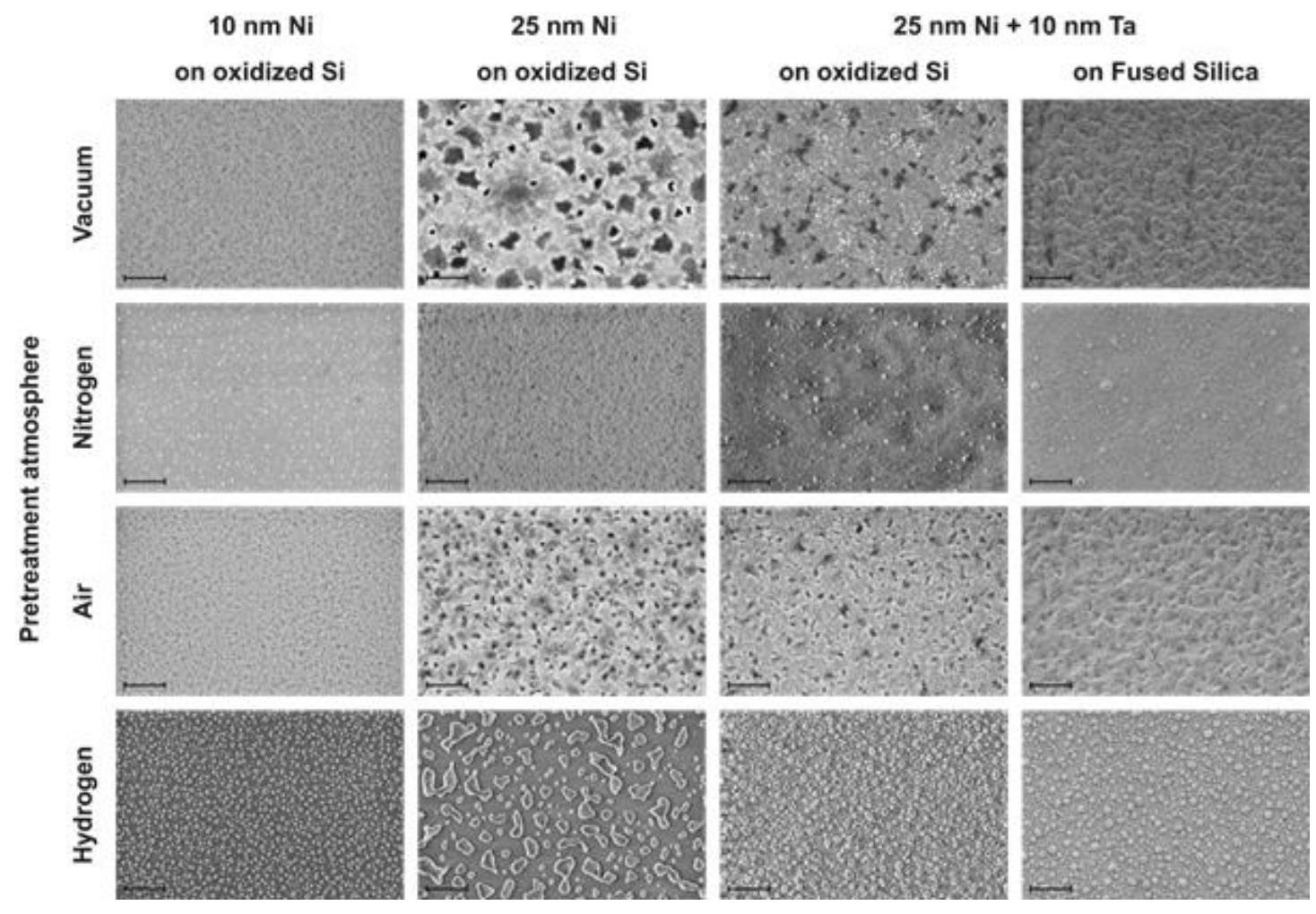

Figure 1: SEM-images of Ni thin films on oxidized silicon and fused silica after pretreatment in different atmospheres $\left(650^{\circ} \mathrm{C}, 1 \mathrm{~h}\right)$. All images have the same scale, i.e. each image represents a sample area of $7.0 \mu \mathrm{m} \times 4.7 \mu \mathrm{m}$ (the scale bars are $1 \mu \mathrm{m})$.

Only pretreatment at $650{ }^{\circ} \mathrm{C}$ for $1 \mathrm{~h}$ in a reducing atmosphere results in the formation of nanoparticles, which can be understood by the fact, besides that these conditions favor dewetting, ${ }^{[40]}$ the reduction of metal oxides enhances surface mobility. ${ }^{[41]}$ The opposite occurs for oxidizing atmosphere, ${ }^{[28,41]}$ for which it is known that even ppm quantities of oxygen or moisture are sufficient to significantly compromise dewetting. ${ }^{[1]}$ This trend is found in Figure 1, where pretreatment in vacuum after evacuation from ambient air, and in technical air results in hole formation, while pretreatment in nitrogen $(99.95 \%)$ leads to the appearance of small hillocks and hydrogen gives separated nanoparticles. 
Examination of the reduced samples in Figure 1 shows that a $10 \mathrm{~nm}$ thick $\mathrm{Ni}$ film yields small particles with a high surface density, in agreement with literature. ${ }^{[20,28,35,36,38-40]}$ The particles have spherical or elliptical shape. For the dewetted $25 \mathrm{~nm} \mathrm{Ni}$ film, the formation of particles, even under reducing conditions, is less clear, and it seems that dewetting either has not been completed, or particles have started to agglomerate, for the applied pretreatment time. The presence of $10 \mathrm{~nm}$ Ta gives a smaller particle size (compared to $25 \mathrm{~nm} \mathrm{Ni}$ without Ta). The shape of the nanoparticles of reduced $25 \mathrm{~nm} \mathrm{Ni} / 10 \mathrm{~nm}$ Ta is elliptical or spherical, similar to dewetted $10 \mathrm{~nm}$ Ni. Based on literature (see Section 3.1 \paragraph 3) it is known that pretreated metal films that have a low surface mobility yield low density, large metallic nanoparticles: since the particle size of reduction-pretreated $25 \mathrm{~nm} \mathrm{Ni}$ is larger than of $25 \mathrm{~nm} \mathrm{Ni} / 10 \mathrm{~nm}$ Ta (exposed to similar pretreatment settings), it is speculated that the surface mobility of $\mathrm{Ni}$ is higher on $\mathrm{Ta}$ than on oxidized silicon. Alternatively, the presence of Ta might initiate an increase in the amount of instabilities in the Ni thin film, which also gives rise to smaller metallic nanoparticles upon reduction-pretreatment. If the arise of spherical metallic nanoparticles is considered as final stage of dewetting (and not the size of spherical nanoparticles), one could argue that the presence of Ta influences (i.e. accelerates) the dewetting process for films exposed to identical pretreatment settings: in case of $25 \mathrm{~nm} \mathrm{Ni}$ on $10 \mathrm{~nm}$ Ta spherical particles are visible after a reduction-pretreatment of $1 \mathrm{~h}$ at 650 ${ }^{\circ} \mathrm{C}$, whereas this is not yet the case of $25 \mathrm{~nm} \mathrm{Ni}$ on oxidized silicon.

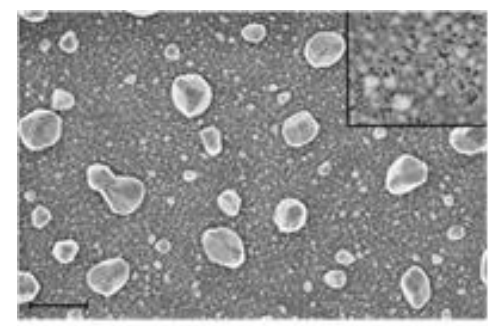

$500{ }^{\circ} \mathrm{C}(2 \mathrm{~h})$

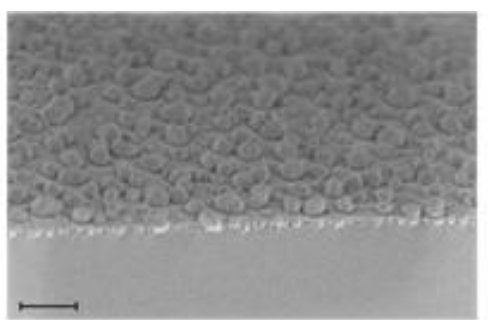

$600{ }^{\circ} \mathrm{C}(1 \mathrm{~h})$

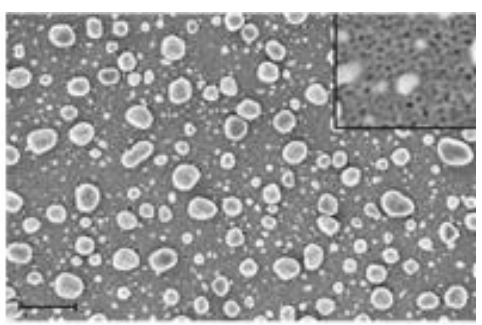

$700^{\circ} \mathrm{C}(1 \mathrm{~h})$

Figure 2: SEM-images of Ni/Ta thin films $(25 \mathrm{~nm} \mathrm{Ni/10} \mathrm{nm}$ Ta) on fused silica after pretreatment in hydrogen atmosphere (the scale bars are $400 \mathrm{~nm}$ ).

As can be concluded from Figure 2, which shows a series of SEM-images of $25 \mathrm{~nm} \mathrm{Ni} / 10 \mathrm{~nm}$ Ta films reduced at various temperatures, the Ta layer does not break 
up into nanoparticles but remains a continuous, porous layer (see insets Figure 2). Comparison of the pretreatments of $25 \mathrm{~nm} \mathrm{Ni} / 10 \mathrm{~nm} \mathrm{Ta}$ at $500{ }^{\circ} \mathrm{C}$ for $2 \mathrm{~h}$ (Figure 2) and $650{ }^{\circ} \mathrm{C}$ for $1 \mathrm{~h}$ (Figure 1) reveals that the thermal budget significantly influences dewetting: $2 \mathrm{~h}$ at $500{ }^{\circ} \mathrm{C}$ yields larger particles (i.e. 2-3 times) than $1 \mathrm{~h}$ at $650{ }^{\circ} \mathrm{C}$, while for a fixed pretreatment time the sample exposed to the highest temperature contains the largest particles.

EDX analysis confirms that the major component of the particles is $\mathrm{Ni}$ (Table 1). The oxygen $\left(\mathrm{O}_{2}\right)$ present in the particles probably arises from exposure to ambient air after pretreatment. Locations between particles show very low Ni content. Unfortunately for areas in-between Ni-particles no discrimination can be made between oxidized silicon and (oxidized) Ta, since the Si-K line and the Ta-M line are both near $1.7 \mathrm{keV} .{ }^{[42,43]}$ No deconvolution of these overlapping EDX peaks was performed.

\begin{tabular}{|c|c|c|c|c|c|c|}
\hline Pretreatment & Location EDX & Element & Line & $\begin{array}{l}\# \\
\text { Counts }\end{array}$ & $\begin{array}{l}\text { Atom } \\
\% \$\end{array}$ & $\begin{array}{l}\text { Element } \\
\text { wt. } \% \S\end{array}$ \\
\hline \multirow{10}{*}{$\begin{array}{l}500^{\circ} \mathrm{C} \\
2 \text { hours }\end{array}$} & \multirow{5}{*}{$\begin{array}{l}\text { In-between } \\
\text { area }\end{array}$} & $\mathrm{C}$ & $\mathrm{K}$ & 1159 & 34 & 21 \\
\hline & & 0 & $\mathrm{~K}$ & 3311 & 28 & 24 \\
\hline & & $\mathrm{Si}$ & $\mathrm{K}$ & 57636 & 37 & 54 \\
\hline & & \multirow{2}{*}{$\mathrm{Ni}$} & $\mathrm{K}$ & 153 & \multirow{2}{*}{1} & \multirow{2}{*}{1} \\
\hline & & & $\mathrm{L}$ & 909 & & \\
\hline & \multirow[t]{5}{*}{ Particle } & C & $\mathrm{K}$ & 407 & 16 & 7 \\
\hline & & 0 & $\bar{K}$ & 3053 & 29 & 16 \\
\hline & & $\mathrm{Si}$ & $\mathrm{K}$ & 26078 & 33 & 33 \\
\hline & & \multirow[b]{2}{*}{$\mathrm{Ni}$} & $\mathrm{K}$ & 5160 & \multirow{2}{*}{22} & \multirow[b]{2}{*}{44} \\
\hline & & & $\mathrm{L}$ & 12748 & & \\
\hline \multirow{10}{*}{$\begin{array}{l}650^{\circ} \mathrm{C} \\
1 \text { hour }\end{array}$} & \multirow{5}{*}{$\begin{array}{l}\text { In-between } \\
\text { area }\end{array}$} & C & $\mathrm{K}$ & 1209 & 34 & 22 \\
\hline & & $\mathrm{O}$ & $\mathrm{K}$ & 3510 & 30 & 25 \\
\hline & & $\mathrm{Si}$ & $\mathrm{K}$ & 52245 & 35 & 51 \\
\hline & & \multirow{2}{*}{$\mathrm{Ni}$} & $\mathrm{K}$ & 301 & \multirow{2}{*}{1} & \multirow{2}{*}{3} \\
\hline & & & $\mathrm{L}$ & 1271 & & \\
\hline & \multirow[t]{5}{*}{ Particle } & C & $\mathrm{K}$ & 240 & 12 & 5 \\
\hline & & 0 & $\mathrm{~K}$ & 2615 & 31 & 16 \\
\hline & & $\mathrm{Si}$ & $\mathrm{K}$ & 18796 & 31 & 29 \\
\hline & & $\mathrm{Ni}$ & K & 4813 & \multirow{2}{*}{26} & \multirow{2}{*}{50} \\
\hline & & $\mathrm{N} I$ & $\mathrm{~L}$ & 12399 & & \\
\hline
\end{tabular}

Table 1: EDX-data on Ni/Ta $(25 \mathrm{~nm} / 10 \mathrm{~nm})$ thin films on oxidized silicon reduced at $500{ }^{\circ} \mathrm{C}(2 \mathrm{~h})$ and $650{ }^{\circ} \mathrm{C}(1 \mathrm{~h})$ : composition of particles, and areas in-between particles. $\left({ }^{\S}\right.$ : relative error $\left.20 \%\right)$.

Table 1 shows that particles formed at $650{ }^{\circ} \mathrm{C}(1 \mathrm{~h})$ have ca. $5 \%$ higher $\mathrm{Ni}$ content than after treatment at $500{ }^{\circ} \mathrm{C}(2 \mathrm{~h})$, in agreement with AFM data (not shown) 
which show taller particles for the former treatment (but with a smaller diameter, as based on top-view SEM images). Because a constant acceleration voltage of $15 \mathrm{keV}$ was used in all EDX measurements, a fixed penetration depth of ca. $3 \mu \mathrm{m}$ can be assumed, so that it is clear that in case of taller Ni particles, less signal will be obtained from the silicon and oxide underneath.

The particle size distribution for samples reduced at $650^{\circ} \mathrm{C}(1 \mathrm{~h})$ is analyzed in detail (Figure 3). A $10 \mathrm{~nm}$ Ni film without Ta yielded smaller particles with a much sharper size distribution (average particle size $55 \mathrm{~nm}$ ) and a 10 times higher surface density than a $25 \mathrm{~nm} \mathrm{Ni}$ film without Ta (average particle size $140 \mathrm{~nm}$ ). A similar difference was found between the $25 \mathrm{~nm} \mathrm{Ni}$ without or with Ta, where ca. 230 particles (per unit area) with broad size distribution and ca. 3000 particles with small size distribution (average particle size $56 \mathrm{~nm}$ ) were found, respectively. The size distribution on $10 \mathrm{~nm} \mathrm{Ni}$ is fairly similar to $25 \mathrm{~nm} \mathrm{Ni} / 10 \mathrm{~nm}$ Ta: the presence of Ta indeed influences dewetting of $\mathrm{Ni}$. No significant differences were found for nanoparticles on fused silica or oxidized silicon.
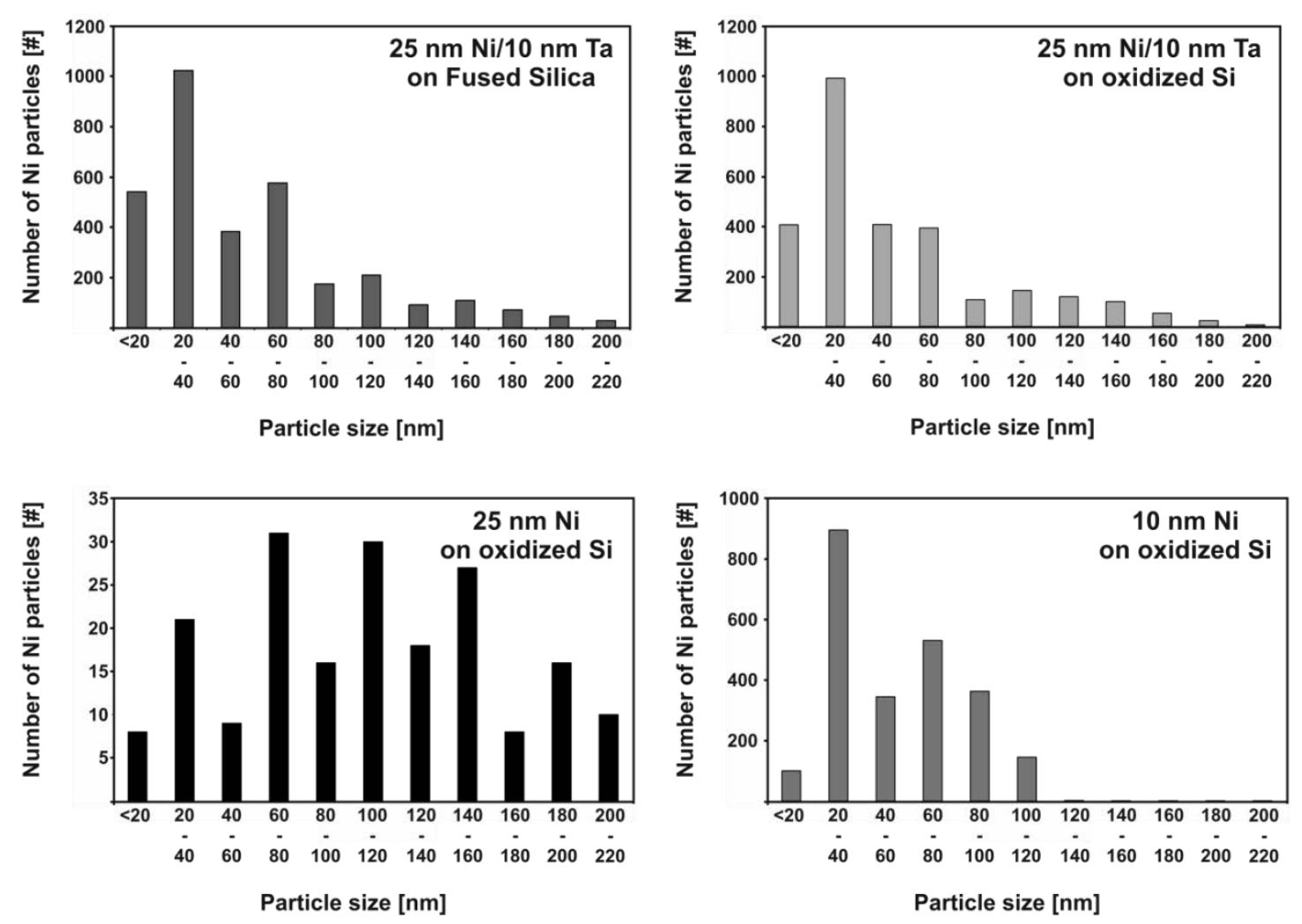

Figure 3: Particle size distributions of Ni thin films reduced at $650{ }^{\circ} \mathrm{C}$ for $1 \mathrm{~h}$. 


\subsubsection{CNF growth on pretreated samples}

Oxidized silicon and fused silica samples containing Ni thin films exposed to different pretreatment atmospheres at $650{ }^{\circ} \mathrm{C}(1 \mathrm{~h})$ were subjected to the CNF synthesis process, as described in the experimental section, the results of which are shown in Figure 4. As expected from the particle results above, no differences were found between fused silica and oxidized silicon as substrate material.

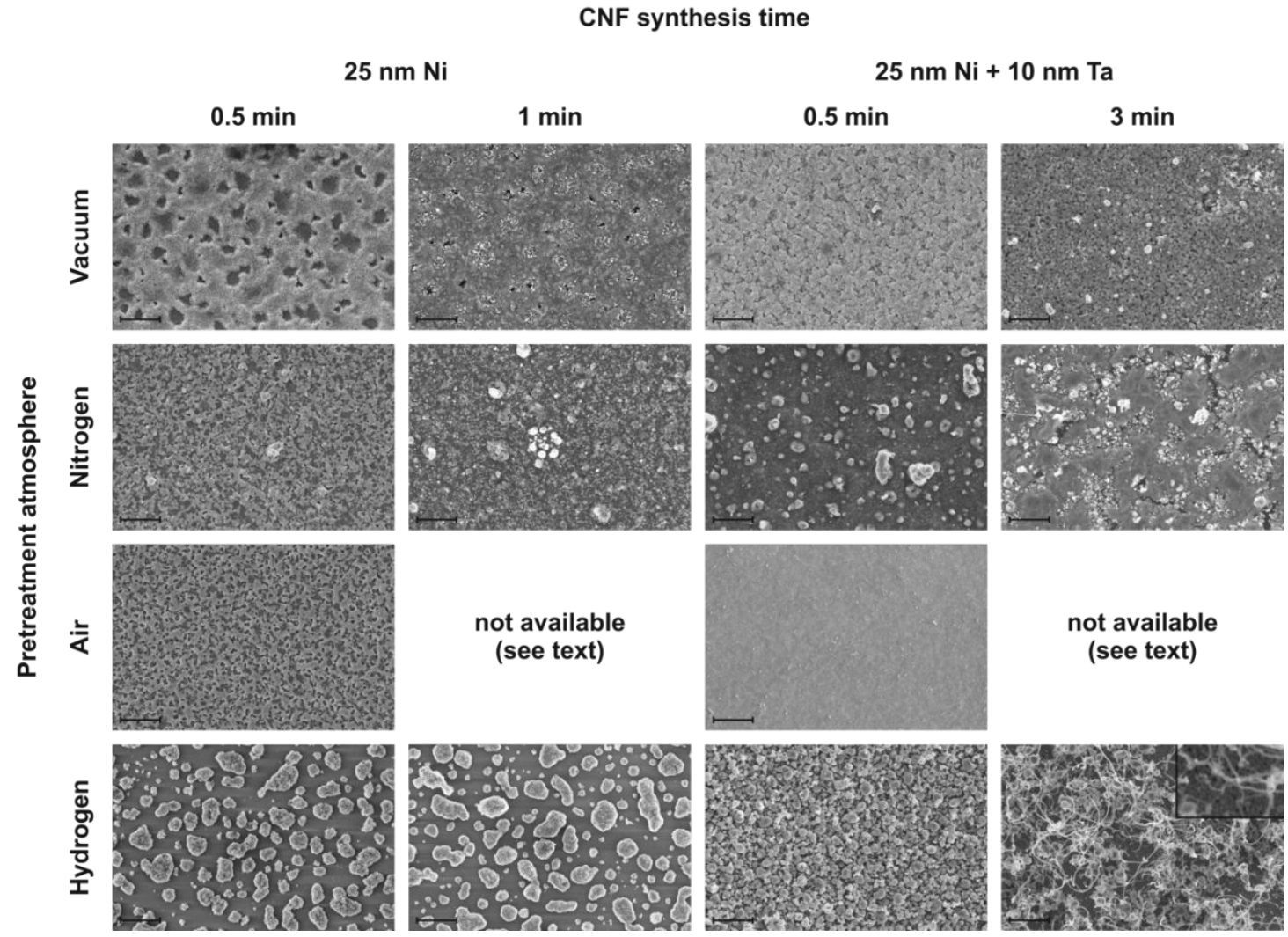

Figure 4: SEM-images of $\mathrm{Ni}$ thin films on oxidized silicon after pretreatment $\left(650{ }^{\circ} \mathrm{C}, 1 \mathrm{~h}\right)$, showing the influence of pretreatment atmosphere and synthesis time on CNF growth. All images have the same scale, i.e. each image represents a sample area of $7.0 \mu \mathrm{m} \times 4.7 \mu \mathrm{m}$ (the scale bars are $1 \mu \mathrm{m})$.

The images reveal no notable difference between vacuum-pretreated samples exposed to 0.5 min CNF synthesis (Figure 4) and samples only pretreated in vacuum (Figure 1), independent of the thin film configuration. Moreover, after $0.5 \mathrm{~min}$ CNF growth on none of the vacuum-pretreated samples traces of carbon were measured with Raman spectroscopy or EDX. On the contrary, after 1 min growth the surface of a $10 \mathrm{~nm}$ Ni film was uniformly covered with many white dots, which turned out to be 
amorphous carbon. For a $25 \mathrm{~nm}$ thick Ni layer and a $25 \mathrm{~nm} \mathrm{Ni} / 10 \mathrm{~nm}$ Ta layer combination these dots were also clearly visible, but their density was lower while their diameter was larger. A CNF synthesis time of 3 min resulted in homogeneous coverage of the metal surface with an amorphous carbon coating (C-coating). In the absence of a Ta layer this C-coating was continuous and prohibited the growth of CNFs, while in the presence of Ta the amorphous carbon layer was porous (spongy) which allowed scarce growth of thin and short fibers (visible as tiny white strikes in Figure 4).

The morphological appearance of nitrogen-pretreated $10 \mathrm{~nm}$ and $25 \mathrm{~nm} \mathrm{Ni}$ films subjected to a CNF growth time of $0.5 \mathrm{~min}$ were nearly identical to the images in Figure 1, except that a thin coating was present on the surface. The presence of Ta underneath the $25 \mathrm{~nm} \mathrm{Ni}$ film resulted in a thicker, continuous amorphous carbon layer with some hillocks (Figure 4), of which the height seemed to increase for a growth-time of $1 \mathrm{~min}$. For a $10 \mathrm{~nm} \mathrm{Ni}$ film, the latter growth time resulted in the formation of spherical and rock-shaped metal particles covered with a thin carbon layer. Compared to nitrogen pretreatment only, significant dewetting of the film occurred during this $1 \mathrm{~min}$ growth step. The morphology of the resulting particles was similar to the appearance of a $25 \mathrm{~nm}$ Ni film pretreated in $\mathrm{H}_{2}$ (see Figure 1), but with a smaller feature size. In contrast, a nitrogen-pretreated $25 \mathrm{~nm}$ Ni film subjected to 1 min CNF growth did not show more dewetting, but was covered with a dense, continuous carbon layer. For the latter case it is concluded that the Ni film was too thick to accomplish dewetting within a period of 1 min CNF growth. For both $10 \mathrm{~nm}$ $\mathrm{Ni}$ and $25 \mathrm{~nm} \mathrm{Ni}$, a growth time of 3 min resulted in a dense, continuous amorphous carbon layer on the surface of the particles $(10 \mathrm{~nm} \mathrm{Ni})$ or the film $(25 \mathrm{~nm} \mathrm{Ni})$, which obstructed the growth of fibers. In case of $\mathrm{Ni} / \mathrm{Ta}$ exposed to a growth time of $3 \mathrm{~min}$, the amorphous carbon layer formed during the first minute of CNF growth had become discontinuous, and a small number of hillocks turned out to be nucleation sites for CNFs (Figure 4).

On samples pretreated with air, a CNF synthesis time of $30 \mathrm{~s}$ yielded a rather thick, homogeneous but porous C-coating on $10 \mathrm{~nm} \mathrm{Ni}$ and $25 \mathrm{~nm} \mathrm{Ni}$, and a thick conformal C-coating on $25 \mathrm{~nm} \mathrm{Ni/10} \mathrm{nm} \mathrm{Ta} \mathrm{(Figure} \mathrm{4).} \mathrm{This} \mathrm{amorphous} \mathrm{C-coating}$ was relatively thick compared to C-layers formed on vacuum and nitrogen-pretreated 
samples exposed to the same CNF synthesis procedure. Whereas for a growth time of 0.5 min no CNFs were observed, for all air-pretreated thin film configurations synthesis times of $\geq 1$ min resulted in excessive CNF growth. ${ }^{[44,45]}$ However, the resulting CNF "carpets" were not attached to the substrate material. ${ }^{[14]}$

When exposed to a CNF growth time of $0.5 \mathrm{~min}$, on all hydrogen-pretreated samples a thin C-coating was visible on only the surface of the particles: individual particles were clearly recognizable, but these were covered with a thin amorphous carbon layer. In contrast to air-pretreated CNF-grown samples, after $30 \mathrm{~s}$ of CNF growth the hydrogen-pretreated $\mathrm{Ni}$ films had no conformal C-layer covering the particles and the areas between the particles (Figure 4). For a CNF synthesis time of 1 min, on $10 \mathrm{~nm} \mathrm{Ni}$ and $25 \mathrm{~nm} \mathrm{Ni}$ the thickness of the C-layer seemed to be somewhat thicker compared to the layer after $0.5 \mathrm{~min} \mathrm{CNF}$ growth, and for the $25 \mathrm{~nm} \mathrm{Ni}$ sample 1 min of CNF synthesis also resulted in the appearance of small white spots in the Ccoating. For 0.5 and $1 \mathrm{~min}$ CNF growth time the presence of $10 \mathrm{~nm}$ Ta underneath 25 $\mathrm{nm} \mathrm{Ni}$ resulted in coverage of the particles with carbon hillocks (visible as gray "balls" in Figure 4). Absence of Ta resulted in release of $\mathrm{Ni}$ thin films from the substrate for a synthesis time of $3 \mathrm{~min}$, while on the hydrogen-pretreated $\mathrm{Ni} / \mathrm{Ta}$ film well-attached CNFs were visible after this growth time (Figure 4). The CNFs were distributed homogeneously on the surface of the sample, randomly oriented, and grown according to tip-type mode. ${ }^{[14]}$ The Ta layer is recognizable as a continuous spongy network underneath the fiber-layer (see inset Figure 4). Moreover, it can be seen that the CNFs had their origin in carbon-coated Ni-particles. The thickness and morphology of this C-layer can be altered by adding hydrogen to the ethylene mixture ${ }^{[15]}$ which also influences the growth rate of CNFs.

Based on HR-SEM images the diameter of the fibers was in the range 15-40 $\mathrm{nm}$, which shows good correlation with the particle size distribution after reduction (Figure 3). The majority of the Ni particles had a diameter below $80 \mathrm{~nm}$. It is concluded from our results that, for the explored experimental settings on $\mathrm{Ni} / \mathrm{Ta}$, spherical nanoparticles with a diameter below $80 \mathrm{~nm}$ are a requisite for fast, i.e. within 3 min, initiation of CNF growth. The CNF diameter range in this work is close to, but a little lower, than the range of 20-50 nm reported earlier for $\mathrm{Ni} / \mathrm{Ta}$ films with an identical as-deposited thickness, ${ }^{[14]}$ the difference being most likely a consequence of 
a different hydrogen-pretreatment step $\left(1 \mathrm{~h}\right.$ at $650{ }^{\circ} \mathrm{C}$ vs. $2 \mathrm{~h}$ at $\left.500{ }^{\circ} \mathrm{C}\right)$ and $\mathrm{CNF}$ synthesis temperature $\left(635^{\circ} \mathrm{C}\right.$ vs. $\left.700{ }^{\circ} \mathrm{C}\right)$. As was shown above, a $2 \mathrm{~h}-500{ }^{\circ} \mathrm{C}$ pretreatment yields larger $\mathrm{Ni}$-nanoparticles than a $1 \mathrm{~h}-650^{\circ} \mathrm{C}$ pretreatment, while it is also known that at higher synthesis temperatures CNFs will also grow on larger nanoparticles (See Section 3.1\Paragraph 2).

The ratio $\mathrm{I}_{\mathrm{D}} / \mathrm{I}_{\mathrm{G}}$ of the relative intensities of the D-band and G-band from the first-order Raman spectrum is traditionally used as an indicator for the degree of graphitization and alignment of fibers. ${ }^{[46,47]}$ For hydrogen-pretreated Ni/Ta samples exposed for $3 \mathrm{~min}$ to the CNF synthesis procedure this ratio was found to be 1.25. Such a value indicates the presence of interstitial defects or quasi-crystalline platelet nature of the CNF layer. ${ }^{[48]}$ Hydrogen-pretreated Ni/Ta samples subjected to longer CNF synthesis times, viz. 10, 30 and $60 \mathrm{~min}$, had an $\mathrm{I}_{\mathrm{D}} / \mathrm{I}_{\mathrm{G}}$-value of $1.20 \pm 0.05$, which shows that the properties of the CNFs are stable throughout the thickness of the CNF layer. Nearly similar values were found for Ni/Ta samples $(25 \mathrm{~nm} / 10 \mathrm{~nm})$ that were hydrogen-pretreated at $500{ }^{\circ} \mathrm{C}(2 \mathrm{~h})$ followed by $1 \mathrm{~h} \mathrm{CNF}$ growth at $700{ }^{\circ} \mathrm{C}$ (use of identical ethylene mixture). ${ }^{[14]}$

\subsection{Conclusions}

In this work the influence of the pretreatment atmosphere and temperature on the dewetting of as-deposited continuous $\mathrm{Ni}$ thin films on oxidized silicon and fused silica and subsequent exposure of the samples to a TC-CVD CNF synthesis procedure was studied. For a pretreatment temperature of $650^{\circ} \mathrm{C}(1 \mathrm{~h})$ in vacuum, air, nitrogen or hydrogen atmospheres only the latter results in the formation of separate nanoparticles. The use of an adhesion layer of $10 \mathrm{~nm}$ Ta underneath the Ni layer influences (i.e. accelerates) the dewetting process of $\mathrm{Ni}$, and it is found that the thermal budget significantly influences the nanoparticle size distribution resulting from the reduction step. EDX-analysis shows that the particles are composed of Ni. No difference in Ni dewetting behavior is found between oxidized silicon and fused silica substrates. In case of reduced $25 \mathrm{~nm} \mathrm{Ni} / 10 \mathrm{~nm}$ Ta the diameter of the resulting nanoparticles has a sharp size distribution with an average of $56 \mathrm{~nm}$. 
On Ni thin film samples pretreated in vacuum or nitrogen and exposed to the CNF synthesis procedure, amorphous carbon layers are formed on metallic surfaces that are not completely dewetted into nanoparticles. Depending on the CNF growth time and the absence/presence of $\mathrm{Ta}$, the carbonaceous layers are continuous (and conformal) or porous (spongy), resulting in hardly any or scarce growth of CNFs. In case of air-pretreated Ni samples, severe release of CNF carpets from the substrate occurs for CNF synthesis times of at least $1 \mathrm{~min}$. It is concluded that pretreatment of Ni thin films in vacuum, nitrogen or air should not be performed when well-attached homogeneous layers of CNFs are desired. Good results are only obtained on reduced Ni films with an adhesion layer of Ta, giving homogeneously distributed, randomlyoriented, well-attached and semi-crystalline CNFs within 3 min synthesis time. The diameter of these fibers is in the range $15-40 \mathrm{~nm}$, which shows good correlation with the particle size distribution after reduction. Absence of the adhesion layer results in the formation of nanoparticles of several hundreds of nanometers diameter on which an amorphous carbon layer is deposited but no CNFs grow for synthesis times up to 3 min. It is found that small spherical nanoparticles with a diameter below $80 \mathrm{~nm}$ are a necessity for fast initiation of CNF nucleation and growth. For the conditions explored in this work this is only the case for reduced $\left(650^{\circ} \mathrm{C}, 1 \mathrm{~h}\right)$ films composed of e-beam evaporated $\mathrm{Ni}(25 \mathrm{~nm})$ and $\mathrm{Ta}(10 \mathrm{~nm})$.

\subsection{References}

[1] S. Iijima, Nature, 1991, 354, 56.

[2] M. M. J. Treacy, T. W. Ebbesen, and J.M. Gibson, Nature, 1996, 381, 678.

[3] S. J. Tans, M. H. Devoret, H. J. Dai, A. Thess, R. E. Smalley, L. J. Geerligs, and C. Dekker, Nature, 1997, 386, 474.

[4] M. Bockrath, D. H. Cobden, P. L. McEuen, N. G. Chopra, A. Zettl, A. Thess, and R. E. Smalley, Science, 1997, 275, 1922.

[5] M. F. Yu, O. Lourie, M. J. Dyer, K. Moloni, T. F. Kelly, and R. S. Ruoff, Science, 2000, 287, 637.

[6] E. Hammel, X. Tang, M. Trampert, T. Schmitt, K. Mauthner, A. Eder, and P. Potschke, Carbon, 2004, 42, 1153. 
[7] J. Kong, N. R. Franklin, C. W. Zhou, M. G. Chapline, S. Peng, K. J.Cho, and H. J. Dai, Science, 2000, 287, 622.

[8] A. M. Fennimore, T. D. Yuzvinsky, W. Q. Han, M. S. Fuhrer, J. Cumings, and A. Zettl, Nature, 2003, 424, 408.

[9] A. C. Dillon, K. M. Jones, T. A. Bekkedahl, C. H. Kiang, D. S. Bethune, and M. J. Heben, Nature, 1997, 386, 377.

[10]P. C. Tsai, S. Pacheco, C. Pirat, L. Lefferts, and D. Lohse, Langmuir, 2009, 25, 12293.

[11] S. S. Fan, M. G. Chapline, N. R. Franklin, T. W. Tombler, A. M. Cassell, and H. J. Dai, Science, 1999, 283, 512.

[12] C. Wang, M. Waje, X. Wang, J. M. Tang, R. C. Haddon, and Y. S. Yan, Nano Lett., 2004, 4, 345.

[13]A. Agiral, A. W. Groenland, J. K. Chinthaginjala, K. Seshan, L. Lefferts, and J. G. E. Gardeniers, J. Phys. D: Appl. Phys., 2008, 41, 194009.

[14]D. B. Thakur, R. M. Tiggelaar, J. G. E. Gardeniers, L. Lefferts, and K. Seshan, Surf. Coat. Technol., 2009, 203, 3435.

[15]D. B. Thakur, R. M. Tiggelaar, J. G. E. Gardeniers, L. Lefferts, and K. Seshan, Chem. Eng. J., 2010, 160, 899.

[16]D. B. Thakur, R. M. Tiggelaar, T. M. C. Hoang, J. G. E. Gardeniers, L Lefferts, and K. Seshan, Appl. Catal. B: Env., 2011, 102, 232.

[17] D. B. Thakur, R. M. Tiggelaar, Y. Weber, J. G. E. Gardeniers, L. Lefferts, and K. Seshan, Appl. Catal. B: Env., 2011, 102, 243.

[18]P. E. Nolan, D. C. Lynch, and A. H. Cutler, Carbon deposition and hydrocarbon formation on group VIII metal catalysts, J. Phys. Chem. B, 1998, 102, 4165.

[19] C. Ducati, I. Alexandrou, M. Chhowalla, G. A. J. Amaratunga, and J. Robertson, J. Appl. Phys., 2002, 92, 3299.

[20] Y. Y. Wei, G. Eres, V. I. Merkulov, and D. H. Lowndes, Appl. Phys. Lett., 2001, 78, 1394.

[21]H. Cui, X. Yang, M. L. Simpson, D. H. Lowndes, and M. Varela, Appl. Phys. Lett., 2004, 84, 4077.

[22] G. S. Choi, Y. S. Cho, S. Y. Hong, J. B. Park, K. H. Son, and D. J. Kim, J. Appl. Phys., 2002, 91, 3847. 
[23]L. Delzeit, C. V. Nguyen, B. Chen, A. Cassell, J. Han, and M. Meyyappan, J. Phys. Chem. B, 2002, 106, 5629.

[24] J. M. Simmons, B. M. Nichols, M. S. Marcus, O. M. Castellini, R. J. Hamers, and M. A. Eriksson, Small, 2006, 2, 902.

[25]A. K. Chakraborthy, J. Jacobs, C. Anderson, C. J. Roberts, and M. R. C. Hunt, J. Appl. Phys., 2006, 100, 084321.

[26] C. J. Lee, J. Park, Y. Huh, and J. Y. Lee, Chem. Phys. Lett., 2001, 343, 33.

[27] Y.-T. Jang, J.-H. Ahn, Y.-H. Lee, and B.-K. Ju, Chem. Phys. Lett., 2003, 372, 745.

[28] S. Pisana, M. Cantoro, A. Parvez, S. Hofmann, A. C. Ferrari, and J. Robertson, Physica E Low Dimens. Syst. Nanostruct., 2007, 37, 1.

[29] L. Dong, J. Jiao, C. Pan, and D. W. Tuggle, Appl. Phys. A, 2004, 78, 9.

[30] J. D. Fowlkes, A. V. Melechko, K. L. Klein, P. D. Rack, D. A. Smith, D. K. Hensley, and M. J. Doktycz, Carbon, 2006, 44, 1503.

[31] R. M. Tiggelaar, R. G. P. Sanders, A. W. Groenland, and J. G. E. Gardeniers, Sens. Actuators A Phys., 2009, 152, 39.

[32] C. H. Bartholomew, Appl. Catal. A, 2001, 212, 17.

[33] M. Chhowalla, K. B. K. Teo, C. Ducati, N. L. Rupesinghe, G. A. J. Amaratunga, A. C. Ferrari, D. Roy, J. Robertson, and W. I. Milne, J. Appl. Phys., 2001, 90, 5308 .

[34] T. de los Arcos, M. G. Garnier, P. Oelhafen, D. Mathys, J. W. Seo, C. Domingo, J. V. García-Ramos, and S. Sánchez-Cortés, Carbon, 2004, 42, 187.

[35]D. P. Burt, W. M. Whyte, J. M. R. Weaver, A. Glidle, J. P. Edgeworth, J. V. Macpherson, and P. S. Dobson, J. Phys. Chem. C Nanomater. Interfaces, 2009, 133, 15133.

[36] G. D. Nessim, D. Acquaviva, M. Seita, and K. P. O’Brien, Adv. Funct. Mater., 2010, 20, 1306.

[37]P. D. Rack, Y. Guan, J. D. Fowlkes, A. V. Melechko, and M. L. Simpson, Appl. Phys. Lett., 2008, 92, 223108.

[38] S. J. Randolph, J. D. Fowlkes, A. V. Melechko, K. L. Klein, H. M. Meyer III, M. L. Simpson, and P. D. Rack, Nanotechnology, 2007, 18, 465304.

[39] J. D. Carey, L. L. Ong, and S. R. P. Silva, Nanotechnology, 2003, 14, 1223. 
[40]A. Geissler, M. He, J.-M. Benoit, and P. Petit, J. Phys. Chem. C Nanomater. Interfaces, 2010, 114, 89.

[41]I. Sushumna and E. Ruckenstein, J. Catal., 1985, 94, 239.

[42] R. C. Weast and M.J. Astle (eds.): CRC Handbook of chemistry and physics, $59^{\text {th }}$ edition; section E: general physical constants, CRC Press Inc., West Palm Beach (FL), USA, 1978.

[43]D. Hrunski, B. Schroeder, M. Scheib, R. M. Merz, W. Bock, and C. Wagner, Thin Solid Films, 2008, 516, 818.

[44] R. T. K. Baker, J. R. Alonzo, J. A. Dumesic, and D. J. C. Yatres, J. Catal., 1982, 77,74 .

[45] N. A. Jarrah, J. G. van Ommen, and L. Lefferts, J. Catal., 2006, 239, 460.

[46] J. K. Chinthaginjala, K. Seshan, and L. Lefferts, Ind. Eng. Chem. Res., 2007, 46, 3968.

[47] N. Larouche and B.L. Stansfield, Carbon, 2010, 48, 620.

[48] A. C. Ferrari, and J. Robertson, Phys. Rev. B Condens. Matter Mater. Phys.,2000, 61, 14095. 


\section{Chapter 4}

\section{A Raman and infrared study of carbon nanofiber growth ${ }^{1}$}

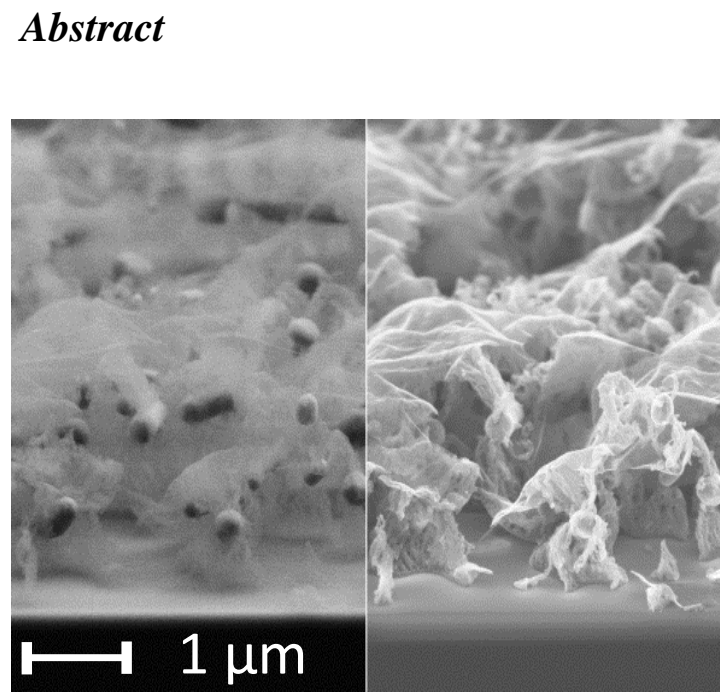

The growth of carbon nanofibers on hydrogen-pretreated oxidized silicon substrates for different synthesis times using ethylene and an ethylene/hydrogen mixture as hydrocarbon sources is studied using Raman and infrared spectroscopy, high resolution scanning electron microscopy and Helium ion microscopy.

\footnotetext{
${ }^{1}$ In preparation for journal submission as "A Raman and infrared study of carbon nanofiber growth" by
} H. Nair, G.R. Cruellas, R.M. Tiggelaar, L. Lefferts, A. van Houselt. 


\subsection{Introduction}

The growth of CNFs by thermal catalytic chemical vapor deposition (TCCVD) is claimed to be a solution-diffusion-precipitation process that generates graphitic carbon. ${ }^{[1]}$ In the present study ethylene and an ethylene/hydrogen mixture is used as a hydrocarbon source and a thin Ni layer as the growth catalyst. In our case the ethylene molecules decompose at the surface of the Ni nanoparticle, and carbon atoms dissolve into the $\mathrm{Ni}$, forming a solid solution, from which upon super-saturation graphite layers nucleate at the nanoparticle surface by diffusion-driven precipitation. ${ }^{[2-4]}$ The effect of nanoparticle size and synthesis temperature on CNF nucleation and growth are investigated in chapter 3. In the present chapter we evaluate the growth in the initial stages of CNF growth on hydrogen pretreated $\mathrm{Ni}$ thin film samples by means of electron and ion microscopy and Raman and infrared spectroscopy. On the basis of the results found in chapter 3, samples of $10 \mathrm{~nm} \mathrm{Ni}$, $25 \mathrm{~nm} \mathrm{Ni}$ and $25 \mathrm{~nm} \mathrm{Ni}$ on $10 \mathrm{~nm} \mathrm{Ta}$, all on $250 \mathrm{~nm} \mathrm{SiO}{ }_{2}$ were selected for a comparative study.

\subsection{Experimental Methods}

\subsubsection{Preparation of nickel thin films on oxidized silicon substrates}

Silicon (Si; (100)-orientation, $p$-type, single-side polished, $100 \mathrm{~mm}$ diameter, $525 \pm 25 \mu \mathrm{m}$ thickness, resistivity 5-10 $\Omega . \mathrm{cm}$; Okmetic, Finland) was used as the support material for the carbon nanofiber (CNF) growth catalysts in this study. A $250 \mathrm{~nm}$ thick layer of $\mathrm{SiO}_{2}$ was grown on the $\mathrm{Si}$ wafer via wet oxidation (45 min, $\left.1000{ }^{\circ} \mathrm{C}\right)$. By standard UV-lithography, a pattern was defined in spin-coated photoresist (Olin, 906-12), resulting in unmasked squares of $8 \mathrm{~mm} \times 8 \mathrm{~mm}$. Subsequently metal thin films were deposited via electron-beam evaporation, resulting in three different sample classes: $10 \mathrm{~nm} \mathrm{Ni}$ on $\mathrm{SiO}_{2} / \mathrm{Si}(001), 25 \mathrm{~nm} \mathrm{Ni}$ on $\mathrm{SiO}_{2} / \mathrm{Si}(001)$ and $25 \mathrm{~nm} \mathrm{Ni}$ on $10 \mathrm{~nm}$ Ta on $\mathrm{SiO}_{2} / \mathrm{Si}(001)$. After the thin film Nicatalyst deposition, an ultrasonic lift-off step in acetone ( $>20 \mathrm{~min}$; VLSI 100038, BASF) was performed, followed by further rinsing and spin drying. Finally, the nickel-coated Si substrates were diced into samples of $1 \mathrm{~cm} \times 1 \mathrm{~cm}$ (Disco DAD-321 
dicing machine). Further details of the sample fabrication procedure can be found elsewhere. ${ }^{[5-7]}$

\subsubsection{Synthesis of CNFs on Ni-coated oxidized silicon substrates}

Prior to CNF synthesis, the substrates were ultrasonically cleaned in acetone (10 min, Branson 200 ultrasonic cleaner) and de-ionized water $\left(5 \mathrm{~min}, 25^{\circ} \mathrm{C}\right.$ ) to remove organic contaminants, followed by drying with pressurized technical air.

For CNF synthesis, the substrates were placed centrally on a flat quartz boat inside a quartz reactor and the temperature was increased in nitrogen $\left(\mathrm{N}_{2} ; 99.999 \%\right.$, INDUGAS) to $500{ }^{\circ} \mathrm{C}\left(5 \mathrm{~K} \mathrm{~min}^{-1}\right)$. At this temperature the substrates were subjected to an in-situ reduction pretreatment for 2 hours (20 vol.\% of hydrogen; 99.999\%, INDUGAS, in $\mathrm{N}_{2}$ at a total flow rate of $50 \mathrm{ml} \cdot \mathrm{min}^{-1}$ ), in order to dewet the continuous as-evaporated thin $\mathrm{Ni}$-film into $\mathrm{Ni}$ nanoparticles which is a prerequisite for catalytic CNF-growth. ${ }^{[6]}$ Subsequently the temperature was increased in $\mathrm{N}_{2}\left(5 \mathrm{~K} \cdot \mathrm{min}^{-1}\right)$ to the CNF-synthesis temperature $\left(635^{\circ} \mathrm{C}\right)$. CNF-synthesis was performed by thermal catalytic chemical vapour deposition of 25 vol.\% ethylene $\left(\mathrm{C}_{2} \mathrm{H}_{4} ; 99.95 \%\right.$ PRAXAIR), with and without addition of 6.25 vol.\% $\mathrm{H}_{2}$, in $\mathrm{N}_{2}$ (total flow rate $100 \mathrm{ml} . \mathrm{min}^{-1}$ ) for various growth times (ranging from 0.5 to 60 minutes). Subsequently the samples were cooled down in $\mathrm{N}_{2}\left(10 \mathrm{~K} \cdot \mathrm{min}^{-1}\right)$ till room temperature.

A schematic cross sectional representation of the three types of CNF substrates used in this study is shown in Figure 1.

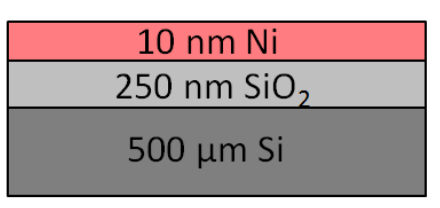

(a)

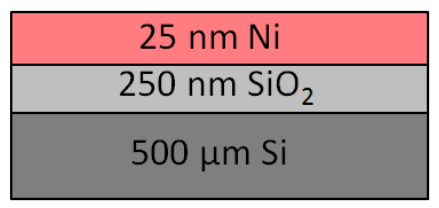

(b)

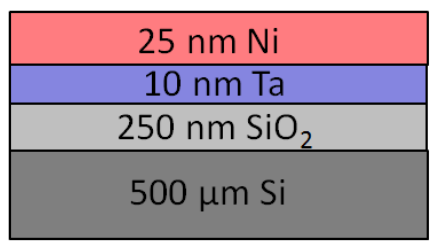

(c)

Figure 1: Schematics of the oxidized silicon substrates with catalyst layer thicknesses of (a) $10 \mathrm{~nm}$ Nickel, (b) $25 \mathrm{~nm}$ Nickel, and (c) $25 \mathrm{~nm}$ Nickel on top of $10 \mathrm{~nm}$ Tantalum.

Thus the synthesis parameters varied in this study are: i) the use of ethylene or ethylene/hydrogen as hydrocarbon source and ii) the synthesis time $(0.5-60 \mathrm{~min})$. The synthesis temperature was kept constant at $635^{\circ} \mathrm{C}$. The as-obtained samples were 
studied by high resolution scanning electron microscopy (HR-SEM) and Helium ion microscopy (HIM) and Raman and infrared spectroscopy.

\subsubsection{SEM \& HIM Imaging}

The synthesized CNF-layers were investigated using high-resolution scanning electron microscopy (HR-SEM; LEO 1550) and Helium ion microscopy (HIM, ultrahigh vacuum (UHV) Orion Plus HIM from Zeiss, equipped with an EverhardtThornley detector for secondary electron detection and a micro-channel plate, situated below the last lens just above the sample, for the qualitative analysis of backscattered helium $^{[8]}$ to determine the presence and morphology of CNFs as well as their uniformity in coverage (top view images). Cross-sectional SEM images were used to determine the thickness of the CNF-layers, and their adhesion to the silicon support.

\subsubsection{Raman Spectroscopy}

The as-synthesized CNF samples were investigated using a Senterra Raman microscope spectrometer (Bruker Optics) equipped with an Olympus BX51 microscope and a Peltier cooled CCD detector, using an excitation wavelength of $532 \mathrm{~nm}(10 \mathrm{~mW})$, a $50 \mathrm{x}$ objective lens, with a numerical aperture of 0.75 , leading to a spot diameter of $\sim 0.9 \mu \mathrm{m}$ and power irradiance of $\sim 1.6 \mathrm{~mW} . \mu \mathrm{m}^{-2}$. The spectra were recorded at room temperature by averaging 10 individual spectra recorded per position on the sample with an accumulation time of 2 seconds each with a resolution of $3-5 \mathrm{~cm}^{-1}$. Spectra from five different sample positions were averaged to account for the heterogeneities in the sample. Before and after recording the spectra the samples were visually checked for damage due to the exposed power density. All spectra were background and baseline corrected and normalized with respect to the Si $520 \mathrm{~cm}^{-1}$ peak.

\subsubsection{Infrared Spectroscopy}

Transmission infrared (IR) spectra of the as-synthesized CNF substrates were recorded using a FTIR spectrometer (Tensor 27, Bruker Optics) with a MCT D316/6$\mathrm{L}$ detector with a resolution of $4 \mathrm{~cm}^{-1}$ in the range of 600 to $4000 \mathrm{~cm}^{-1}$. Spectra of the corresponding Ni-coated (10 or $25 \mathrm{~nm} \mathrm{Ni}$ or $25 \mathrm{~nm} \mathrm{Ni}$ on $10 \mathrm{~nm} \mathrm{Ta}$, see Figure 1) 
oxidised silicon substrates prior to CNF growth were used as background. All spectra were shown baseline corrected.

\subsection{Results and Discussion}

\subsubsection{SEM \& HIM Imaging}

We first describe the observations made by using SEM and HIM imaging techniques. Figure 2 shows cross-sectional SEM images of $10 \mathrm{~nm} \mathrm{Ni}$ (Figure 2a-e), $25 \mathrm{~nm} \mathrm{Ni}$ (Figure 2f-j) and $25 \mathrm{~nm} \mathrm{Ni} / 10 \mathrm{~nm}$ Ta (Figure 2k-o) substrates after growth in ethylene for $0,0.5,1,3$ and 60 min growth time.

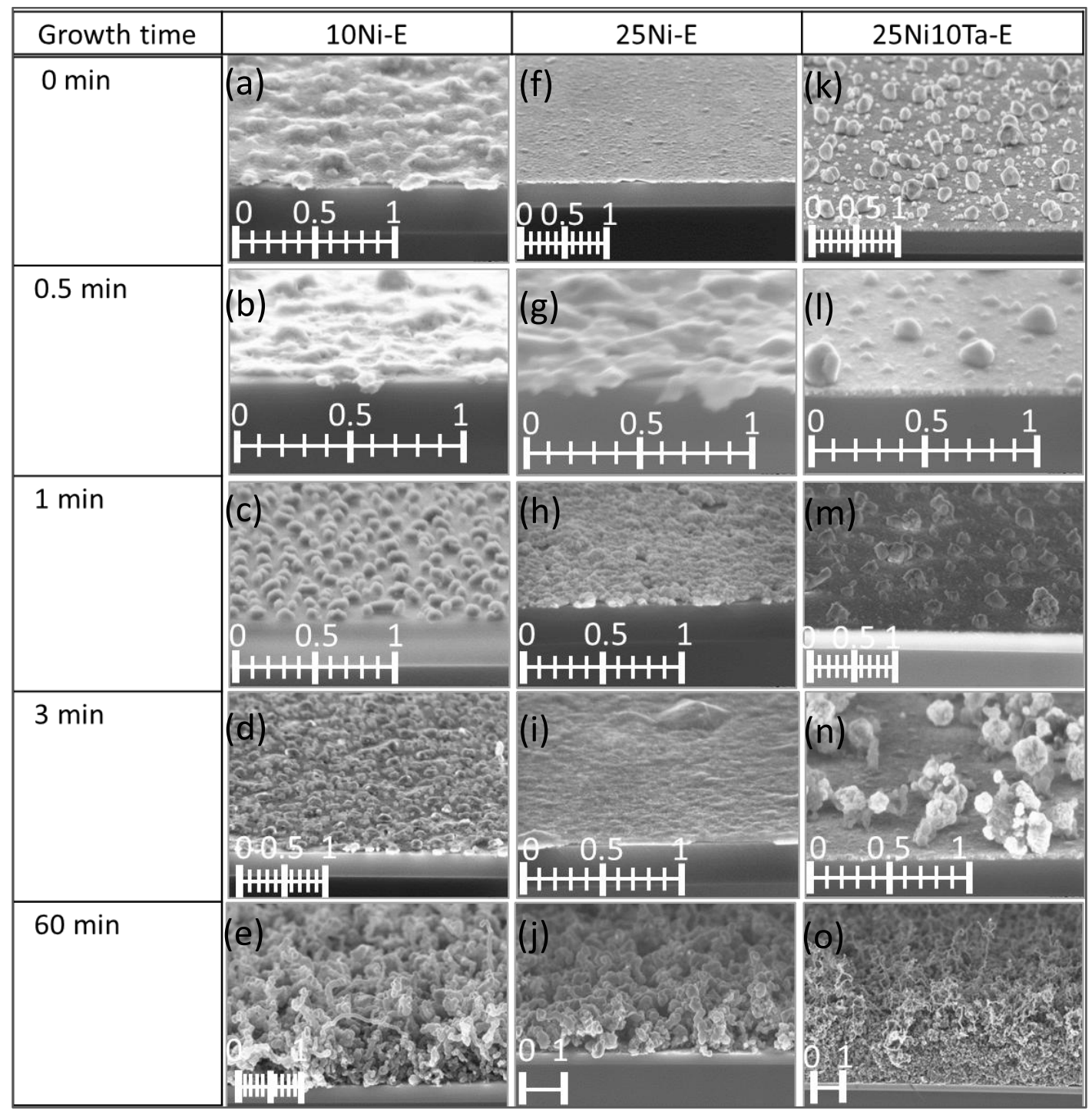

Figure 2: SEM-images after 0, 0.5, 1, 3 and 60 min of CNF growth on substrates with $10 \mathrm{~nm}$ Nickel [(a)-(e)], $25 \mathrm{~nm}$ Nickel [(f)-(j)], and $25 \mathrm{~nm}$ Nickel on top of $10 \mathrm{~nm}$ Tantalum [(k)-(o)]. 


\section{Chapter 4}

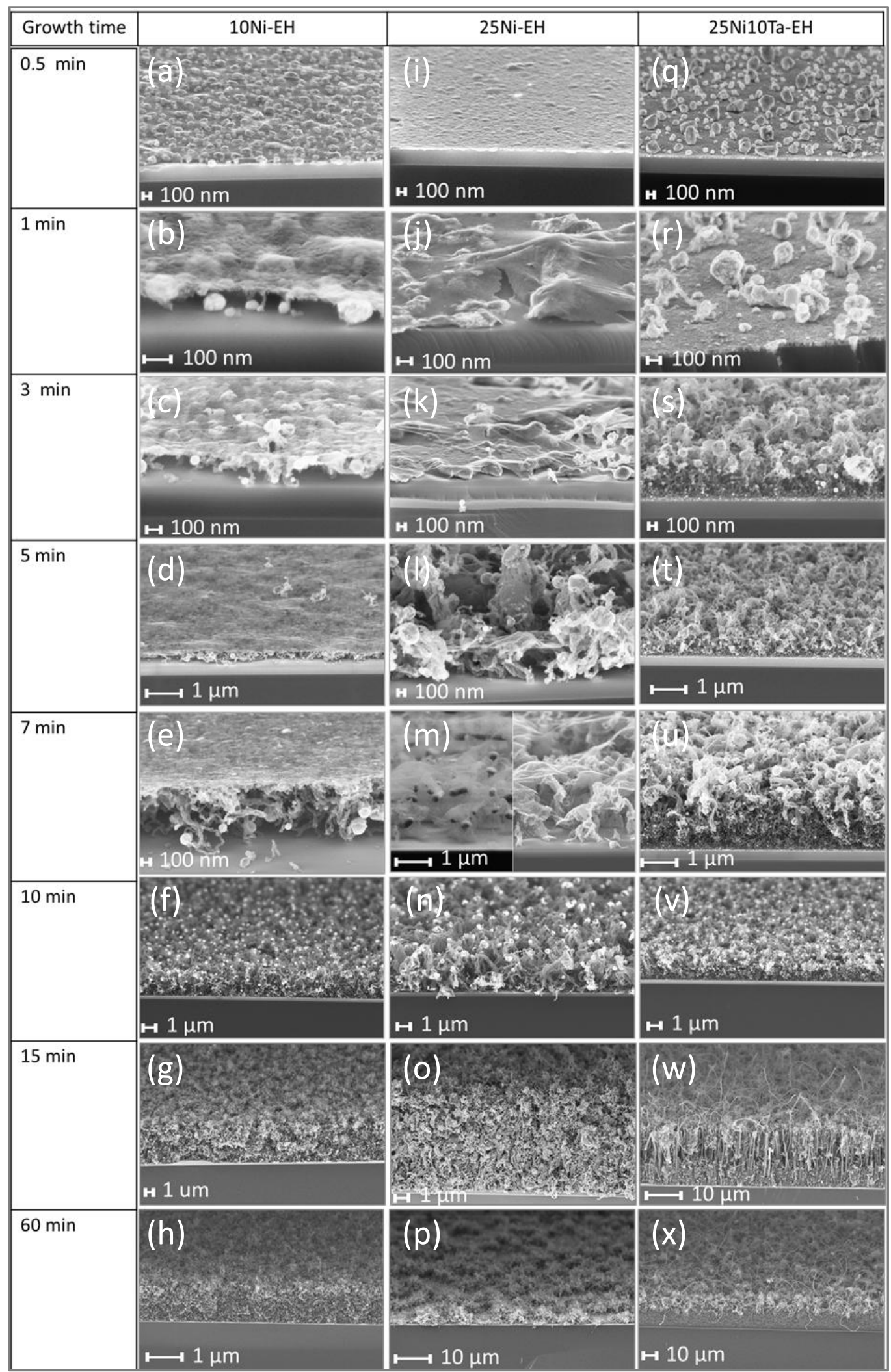

Figure 3: Cross-sectional SEM-images of $10 \mathrm{~nm}$ Ni [Figs. 3(a)-(h)], $25 \mathrm{~nm} \mathrm{Ni} \mathrm{[Figs.} \mathrm{3(i)-(p)]} \mathrm{and}$ $25 \mathrm{~nm} \mathrm{Ni} / 10 \mathrm{~nm}$ Ta [Figs. 3(q)-(x)] substrates after growth in the ethylene/hydrogen mixture for $0.5,1,3,5,7,10,15$ and 60 min growth time. 
On all substrates the thin Ni film has fragmented into small particles, which in the case of the 10 and $25 \mathrm{~nm}$ Ni layers are covered by a "carpet" layer for the initial growth times, see for instance, Figure 2a, $\mathbf{b}$ and $\mathbf{g}$, while this layer is absent after 60 minutes growth, see Figure $2 \mathbf{e}$ and $\mathbf{j}$. On the $25 \mathrm{~nm} \mathrm{Ni} / 10 \mathrm{~nm}$ Ta sample there is no carpet layer observed. After 60 minutes of growth time on all samples a CNF layer is observed, with layer heights of $\sim 1 \mu \mathrm{m}$ for the 10 and $25 \mathrm{~nm}$ Ni sample, while for the $25 \mathrm{~nm} \mathrm{Ni} / 10 \mathrm{~nm}$ Ta sample the layer height is $\sim 3.7 \mu \mathrm{m}$.

Figure 3 shows cross-sectional SEM images of $10 \mathrm{~nm}$ Ni (Figure 3a-h), 25 $\mathrm{nm} \mathrm{Ni} \mathrm{(Figure} \mathrm{3i-p)} \mathrm{and} 25 \mathrm{~nm} \mathrm{Ni} / 10 \mathrm{~nm}$ Ta (Figure 3q-x) substrates after growth in the ethylene/hydrogen mixture for $0.5,1,3,5,7,10,15$ and 60 min growth time. Addition of hydrogen to the growth mixtures results clearly in a faster growth rate, since already in the first minutes CNF growth can be observed resulting in CNF layers of a few micrometers in height after $7 \mathrm{~min}$ growth, to layers of several tens of micrometers after an hour growth. A remarkable difference in the first few minutes of growth can be observed between the $10 / 25 \mathrm{~nm}$ Ni samples on one hand and the 25 $\mathrm{nm} \mathrm{Ni}$ on $10 \mathrm{~nm}$ Ta sample on the other hand. For the former samples a distinct thin layer is observed on top of the CNFs for $1-7$ minutes of growth time, see Figure 3be and $\mathbf{j}-\mathbf{m}$, while such a layer is clearly absent for the corresponding growth times of the latter sample, Figure 3r-u. This thin layer is flexible; since it easily stretched without breaking apart, see Figure $\mathbf{3 j}$ and $\mathbf{k}$. In addition, it is obviously thin, as it is almost transparent for the secondary electrons which form the SEM images, see Figure 3b-e and j-m.

What exactly is this layer and why is it only observed for the $10 / 25 \mathrm{~nm} \mathrm{Ni}$ layers and not for the sample with Ta? In an attempt to answer this questions Helium ion microscopy was performed as well as Raman (see section 4.3.2) and infrared spectroscopy (see section 4.3.3). Helium ion microscopy exhibits a high surface sensitivity, in particular for thin carbon layers. ${ }^{[9]}$ Figure 4 shows HIM images (obtained using an acceleration voltage of $15 \mathrm{kV}$ ) of a $25 \mathrm{~nm} \mathrm{Ni}$ sample after 7 minutes growth time in an ethylene/hydrogen mixture. The secondary electron image, displayed in Figure 4a, reflects the surface morphology very accurately due to the small escape depth of the secondary electrons. Figure $\mathbf{4 b}$ displays a simultaneously recorded image of the backscattered helium. Dark areas in this image 
correspond to light elements (having a low backscatter probability), while bright areas correspond to heavy elements (with a high backscatter probability). In Figure 4a indeed the thin layer is clearly observable, while in Figure $\mathbf{4 b}$ the thin film isn't clearly visible and both rod-like and more spherical white particles (the Ni particles from which the CNFs grow) are visible. From this comparison it is evident that the thin film is comprised of light element, most likely carbon.
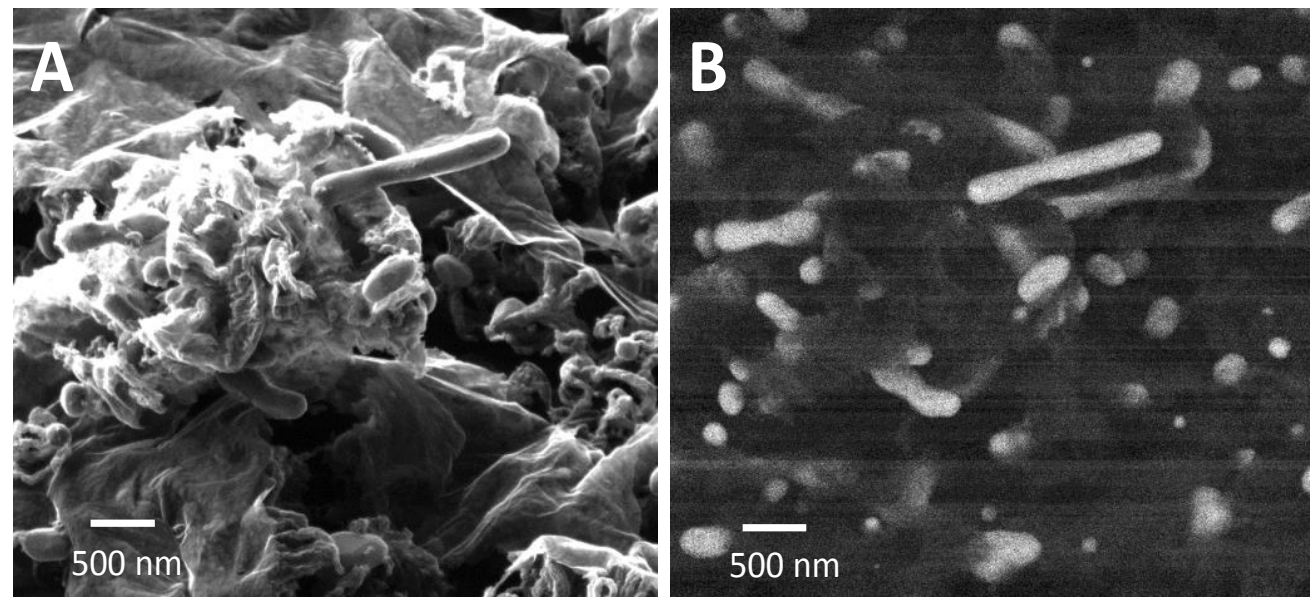

Figure 4: HIM-images of $25 \mathrm{~nm} \mathrm{Ni} \mathrm{sample} \mathrm{after} 7$ minutes of growth in an ethylene/hydrogen mixture. (a) displays the secondary electron image, (b) the backscattered helium image.

\subsubsection{Raman spectroscopy}

To verify the hypothesis that the thin layer is comprised of carbon, Raman and IR spectroscopy was performed. Figure 5 displays Raman spectra of the $10 \mathrm{~nm} \mathrm{Ni}$ (Figure 5a and d), $25 \mathrm{~nm} \mathrm{Ni}$ (Figure $5 \mathbf{b}$ and e) and $25 \mathrm{~nm} \mathrm{Ni} / 10 \mathrm{~nm} \mathrm{Ta}$ (Figure 5c and f) substrates after growth in an ethylene (Figure 5a-c) or in an ethylene/hydrogen mixture (Figure 5d-f), for the indicated growth times. All the spectra are normalized w.r.t. the Si $520 \mathrm{~cm}^{-1}$ peak, thus reflecting changes in the composition and structure of the top layers of the samples.

In all the spectra in Figure 5 the characteristic Si peaks at 300, 518 and 942$980 \mathrm{~cm}^{-1}$ are observed. ${ }^{[10]}$ In addition, for $t>0$, in all the spectra the characteristic bands for graphitic carbon are observed, confirming the presence of carbon in all samples for $t>0$. We discuss the most prominent ones shortly, following the relevant 
literature. ${ }^{[11-13]}$ In the first order part of the spectrum, up to $\sim 1800 \mathrm{~cm}^{-1}$, where one photon is scattered, the $G$ band around $1580 \mathrm{~cm}^{-1}$ and the $D$ band around $1350 \mathrm{~cm}^{-1}$ are clearly observed in all spectra. The $G$ band with its physical origin in the excitation of an optical phonon mode with $E_{2 \mathrm{~g}}$ symmetry, which is active in unperturbed graphite planes ${ }^{[11]}$ is a clear indication of the presence of $s p^{2}$ hybridized carbon, while the $D$ band, which is associated with the in- plane breathing mode of the carbon hexagonal ring with $A_{1 \mathrm{~g}}$ symmetry, is only observed in defected and nanocrystalline graphitic materials. ${ }^{[14]}$ The integrated intensity ratio of the $D$ and $G$ band, which is widely used as figure of merit for the defect quantity of graphitic materials isn't analysed in detail here. In addition, a longitudinal optical phonon mode (LO) is observed in between the $D$ and $G$ band, around $1450 \mathrm{~cm}^{-1}$, which in the spectra in Figure 5 is not visible as a separate peak, but as variation in the height of the "valley" between the $D$ and $G$ band. The physical origin of the shoulder observed $\sim 1125 \mathrm{~cm}^{-1}$ is not precisely known. It could be related to the presence of $\mathrm{sp}^{3}$ hybridized carbon. ${ }^{[15,16]}$

In the second order part of the spectrum, where two phonons are used for excitation, clear peaks are observed around 2330, 2455, 2627, 2651, 2685, 2930 and $3215 \mathrm{~cm}^{-1}$. Not all this signals are related to carbonaceous material. The peak observed at $2329 \mathrm{~cm}^{-1}$ originates from the Raman mode of $\mathrm{N}_{2}$ gas, which is present in the air surrounding the sample. ${ }^{[17]}$ The peak at $2455 \mathrm{~cm}^{-1}$, which is most prominent in the 60 min growth samples in Figure 5a-c, is most likely a combination of the shoulder at $\sim 1125 \mathrm{~cm}^{-1}$ and the $D$ band ${ }^{[18]}$. The origin of the $2651 \mathrm{~cm}^{-1}$ and the $2627 \mathrm{~cm}^{-1}$ signal, which is only observed at short growth times [till $3 \mathrm{~min}$ in Figure 5a-c and e, till 1 min in Figure $5 \mathbf{f}$ and absent in Figure 5d is not precisely known. We are not aware of any studies observing this peak in carbon samples. Most likely it should be attributed to a form of silicon carbide. ${ }^{[19]}$ The peaks at $\sim 2700, \sim 2900$ and $\sim 3200 \mathrm{~cm}^{-1}$ are the overtones of the $D$ band, the LO mode and the $G$ band, respectively (note indeed the correlation in intensity between the peak $\sim 2700 \mathrm{~cm}^{-1}$ and the height of the valley between the $D$ and $G$ band in the first order part of the spectrum). Note that in all samples the intensity of the $2 D$ band at $2700 \mathrm{~cm}^{-1}$ after 60 minutes growth time is significantly higher than for the lower growth times. This $2 D$ 
band is reported to be structure sensitive and can provide information related to tortuosity and crystalline nature of materials. ${ }^{[12]}$
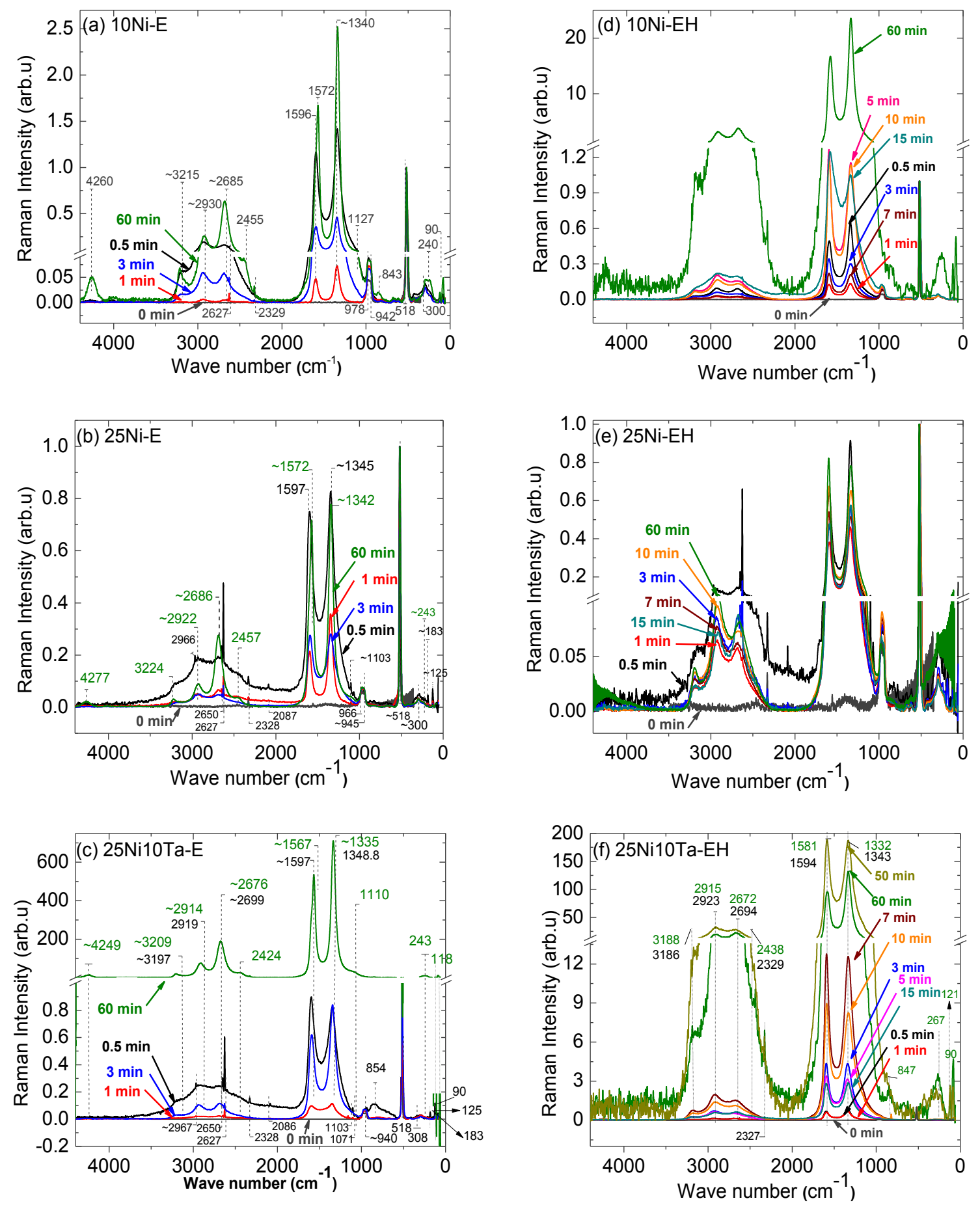

Figure 5: Raman spectra of the $10 \mathrm{~nm} \mathrm{Ni}$ [(a) and (d)], $25 \mathrm{~nm} \mathrm{Ni} \mathrm{[(b)} \mathrm{and} \mathrm{(e)]} \mathrm{and} 25 \mathrm{~nm} \mathrm{Ni/10} \mathrm{nm}$ Ta $[(c)$ and (f)] substrates after growth in ethylene $[(a)-(c)]$ or in an ethylene/hydrogen mixture $[(d)-(f)]$. For each spectrum the corresponding growth time is indicated. 
A peak $\sim 4250 \mathrm{~cm}^{-1}$ is observed in the 60 min samples. This peak is mostly clearly present in the samples grown with ethylene only (Figure 5a-c). This band was also observed in HOPG and was assigned as $2 D+G$ overtone. ${ }^{[19,20]}$ Although clearly a more profound analysis of the here described Raman spectra is needed, some remarks about the growth of CNFs and the observed layer can be made here. Firstly, since in all Raman spectra clear graphitic carbon signals are observed, it's very likely that the observed layer is indeed comprised of carbon. Second, there is no obvious difference between the Raman spectra from the $10 / 25 \mathrm{~nm}$ Ni samples on the one hand versus the spectra from the $25 \mathrm{~nm} \mathrm{Ni}$ on $10 \mathrm{~nm}$ Ta at the other hand. Third, in all cases the peaks present at 2627 and $2651 \mathrm{~cm}^{-1}$ hint towards the presence of silicon carbides during the initial stages of the growth.

\subsubsection{Infrared Spectroscopy}

Infrared spectra are also obtained to verify the hypothesis that the thin layer is comprised of carbon. All the substrates used in this study have silicon as the base material. Hence the signal obtained from silicon is used as background for the sample spectra in different stages of the CNF growth.

Figure 6 displays the IR spectra, corrected by a subtraction of the corresponding $10 \mathrm{~nm} \mathrm{Ni,} 25 \mathrm{~nm} \mathrm{Ni}$ or $25 \mathrm{~nm} \mathrm{Ni} / 10 \mathrm{~nm}$ Ta signal (hence displaying difference spectra) for the $10 \mathrm{~nm} \mathrm{Ni}$ (Figure 6a and d), $25 \mathrm{~nm} \mathrm{Ni}$ (Figure 6b and e) and $25 \mathrm{~nm} \mathrm{Ni} / 10 \mathrm{~nm}$ Ta (Figure 6c and f) substrates after growth in ethylene (Figures 6a-c) or in an ethylene/hydrogen mixture (Figures 6d-f) for the indicated growth times.

From all the peaks observed in Figure 6 we list the most prominent ones shortly here and remark that a more profound and detailed analysis of the peak intensities is in preparation.

In all the spectra, the characteristic peak of silicon dioxide is seen around 1095 $\mathrm{cm}^{-1}$ corresponding to the asymmetric stretching mode of the non-linear Si-O-Si quasimolecule with $\mathrm{C}_{2 v}$ symmetry ${ }^{[21]}$. Clear differences are observed for the intensity of this peak, compare Figures $6 \mathbf{a}$ and $\mathbf{d}$ at one hand, where this peak is negative to Figures $\mathbf{6 b} / \mathbf{c}$ and $\mathbf{e} / \mathbf{f}$ at the other hand where this peak is positive. 

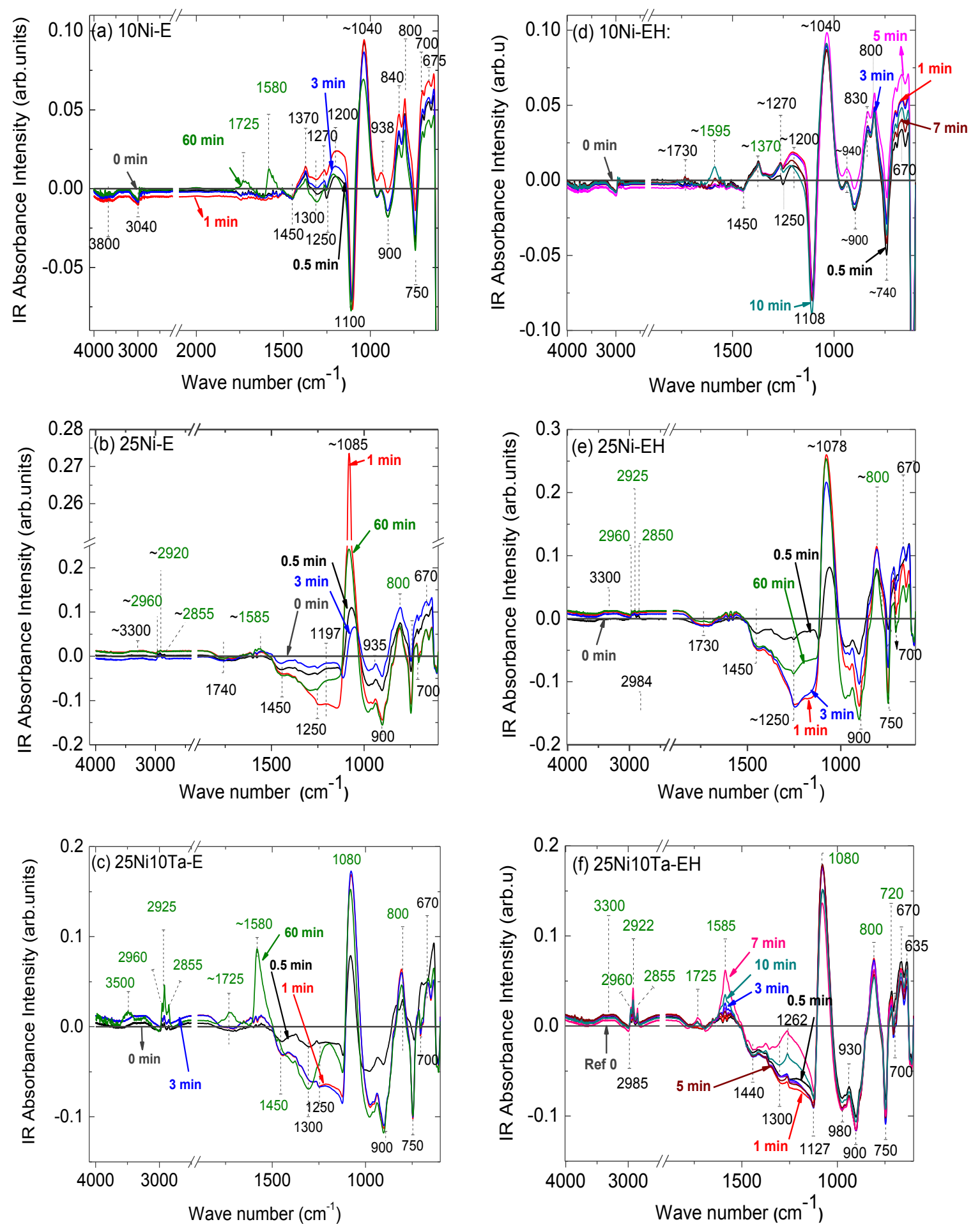

Figure 6: Infrared spectra of the $10 \mathrm{~nm} \mathrm{Ni}$ [(a) and (d)], $25 \mathrm{~nm} \mathrm{Ni}$ [(b) and (e)] and $25 \mathrm{~nm} \mathrm{Ni/10}$ nm Ta [(c) and (f)] substrates after growth in ethylene [(a)-(c)] or in an ethylene/hydrogen mixture $[(d)-(f)]$. For each spectrum the corresponding growth time is indicated. 
A negative peak would imply that $\mathrm{SiO}_{2}$ has disappeared during growth, while a positive peak would imply growth of the $\mathrm{SiO}_{2}$ layer. One should, however, bear into mind that these spectra are taken at physically different samples, since the reference sample is obviously not used for CNF growth. Variations of the thickness of the $\mathrm{SiO}_{2}$ layer between different samples will hence also show up as variations of the 1095 $\mathrm{cm}^{-1}$ peak height and are proposed here as the origin of the variations observed for this peak in Figure 6.

A positive peak $\sim 800 \mathrm{~cm}^{-1}$ is visible in all samples (Figure 6a-f), irrespective of substrate type or reaction mixture combination. This peak intensity was nearly similar ( 0.05) for $10 \mathrm{~nm} \mathrm{Ni}$ (Figure 6a and d) and $25 \mathrm{~nm} \mathrm{Ni-10} \mathrm{nm}$ Ta (Figure 6c and f) while slightly greater $(\sim 0.1)$ in $25 \mathrm{~nm}$ Ni-E (Figure 6b), especially for $3 \mathrm{~min}$ and for $1 \mathrm{~min}$ and $3 \mathrm{~min}$ with ethylene/hydrogen (Figure 6e) and could possibly be attributed to the $\beta-\mathrm{SiC}^{[22]}$. We also observe peaks near $935 \mathrm{~cm}^{-1}$ and a negative peak $900 \mathrm{~cm}^{-1}$ in all samples. It is reported that the isolated aromatic C-H out-of-plane bending can give rise to peaks around $870 \mathrm{~cm}^{-1} .{ }^{[23]}$ Peaks around $750 \mathrm{~cm}^{-1}$ could possibly originate from $\mathrm{Si}-\mathrm{CH}_{\mathrm{n}}(n=2,3)$ vibrations. ${ }^{[24]}$

A peak $\sim 1585 \mathrm{~cm}^{-1}$ seems to develop in most of the spectra in Figure 6 and is especially clear and intense in Figure 6a, c, $\mathbf{d}$ and $\mathbf{f}$. Intensity analysis shows that the intensity of this peak increases almost linearly with CNF layer thickness indicating that this peak arises from CNFs and can be assigned to the aromatic ring stretch of carbon skeleton which was also observed around $1570-1580 \mathrm{~cm}^{-1}$ by Ros et.al. ${ }^{[23,25]}$

The peak near $1725 \mathrm{~cm}^{-1}$ was clearly visible in $60 \mathrm{~min}$ spectra of $10 \mathrm{~nm}$ Ni-E (Figure 6a) and in $25 \mathrm{~nm} \mathrm{Ni} / 10 \mathrm{~nm} \mathrm{Ta}$-E \& EH (Figure 6c and f). In $25 \mathrm{~nm} \mathrm{Ni} / 10$ $\mathrm{nm}$ Ta samples, this peak intensity increased with an increase in growth time and was proportional to the CNF layer thickness. Ros et. al. observed this peak around 1717$1712 \mathrm{~cm}^{-1}$ in $\mathrm{CNFs}$ and assigned it as the $\mathrm{C}=\mathrm{O}$ stretching vibration from lactone, ketone (carbonyl) or carboxylic groups, or any surface oxygen complexes, which can be present in CNFs. ${ }^{[23-28]}$

A group of three peaks at 2855,2923 and $2960 \mathrm{~cm}^{-1}$ were clearly visible in the $25 \mathrm{~nm} \mathrm{Ni} / 10 \mathrm{~nm} \mathrm{Ta}-\mathrm{E}$ and EH samples: ethylene-grown CNFs showed this signal at $3 \mathrm{~min}$ and $60 \mathrm{~min}$ (see Figure 6c), ethylene-hydrogen grown CNFs showed these 
peaks from 0.5 to $60 \mathrm{~min}$ (Figure 6f). The intensity of these peaks was proportional to the increasing CNF layer thickness indicating that these peaks originate from CNFs. The $\mathrm{CH}_{2} / \mathrm{CH}_{3}$ stretching vibrations can lead to the peaks around 2850 (symmetric vibration of $\mathrm{CH}_{2}$ ), 2925 (antisymmetric vibration of $\mathrm{CH}_{2}$ ) and $2960 \mathrm{~cm}^{-1}$ (antisymmetric stretching vibration of $\mathrm{CH}_{3}$ ). ${ }^{[23]}$ These peaks are attributed to the partial conversion from $\mathrm{sp}^{2}$ to $\mathrm{sp}^{3}$-hybridized carbon forming $\mathrm{C}-\mathrm{H}$ bonds as a result of interaction between hydrogen and carbon on the edges of CNFs $\left(\mathrm{sp}^{2}\right.$ and $\mathrm{sp}^{3}$ bonded carbon at 2960 and $2855 / 2923$ respectively). ${ }^{[23,29-31]}$ Remarkably this trio of peaks is most intense in the samples with Ta (compare Figure $\mathbf{6 c}$ and $\mathbf{f}$ with the others), in which case we didn't observe the "carpet" layer in the SEM and HIM images.

In short, in the IR spectra shown in Figure 6, certainly CNF related signals can be observed and a higher amount of $\mathrm{sp}^{3}$ hybridized carbon seems to be present in the samples without a "carpet" layer. More profound analysis is however needed to relate the microscopic and spectroscopic data conclusively.

\subsection{Conclusions}

In this chapter, we studied the growth of CNFs on $10 \mathrm{~nm} \mathrm{Ni}, 25 \mathrm{~nm} \mathrm{Ni}$ and $25 \mathrm{~nm} \mathrm{Ni} / 10 \mathrm{~nm}$ Ta on oxidized silicon substrates. SEM images show the presence of a "carpet" layer, especially at shorter growth time on $10 \mathrm{~nm} \mathrm{Ni}$ and $25 \mathrm{~nm} \mathrm{Ni}$, whereas this is completely absent in $25 \mathrm{~nm} \mathrm{Ni} / 10 \mathrm{~nm} \mathrm{Ta}$, probably due to the dewetting properties of tantalum. HIM confirms that the "carpet" layer is made up of a light element, like carbon. Presence of characteristic signals of carbon in the Raman spectra and Infrared spectra are also in accordance with an interpretation that the carpet layer is comprised of carbon. $10 \mathrm{~nm} \mathrm{Ni}$ and $25 \mathrm{~nm}$ Ni behaved similarly as evidenced from the SEM images and the observed similarity in Raman and IR spectra. In addition, from the Raman spectra we suspect that silicon carbide is present in all samples. Addition of hydrogen enhanced the CNF growth and initiated faster nucleation and growth even in shorter growth times resulting in intense carbon signals in corresponding Raman and IR spectra. 


\subsection{References}

[01] P. E. Nolan, D. C. Lynch, and A. H. Cutler, Carbon deposition and hydrocarbon formation on group VIII metal catalysts, J. Phys. Chem. B, 1998, 102(21), 4165.

[02] C. Ducati, I. Alexandrou, M. Chhowalla, G. A. J. Amaratunga, and J. Robertson, J. Appl. Phys., 2002, 92, 3299

[03] Y. Y. Wei, G. Eres, V. I. Merkulov, and D. H. Lowndes, Appl. Phys. Lett., 2001, 78, 1394.

[04] H. Cui, X. Yang, M. L. Simpson, D. H. Lowndes, and M. Varela, Appl. Phys. Lett., 2004, 84, 4077.

[05] H. Nair, R. M. Tiggelaar, D. B. Thakur, J. G. E. Gardeniers, A. van Houselt, and L. Lefferts, Chem. Eng. J, 2013, 227, 56.

[06] R. M. Tiggelaar, D. B. Thakur, H. Nair, L. Lefferts, K. Seshan, and J. G. E. Gardeniers, Thin Solid Films, 2013, 534, 341.

[07] D. B. Thakur, R. M. Tiggelaar, J. G. E. Gardeniers, L. Lefferts, and K. Seshan, Surf. Coat. Technol., 2009, 203, 3435.

[08] R. van Gastel, L. Barriss, C. Sanford, G. Hlawacek, L. Scipioni, A. Merkle, D. Voci, C. Fenner, H. J. W. Zandvliet, and B. Poelsema, Microsc. Microanal, 2011, 17, 928.

[09] G. Hlawacek, V. Veligura, S. Lorbek, T.F. Mocking, A. George, R. van Gastel, H. J. W. Zandvliet, and B. Poelsema, Beilstein J. Nanotechnol. 2012, 3, 507.

[10] A. Susarrey-Arce, R. M. Tiggelaar, R. G. P. Sanders, B. Geerdink, L. Lefferts, J. G. E. Gardeniers, and A. van Houselt, J. Phys. Chem. C, 2013, 117, 21936.

[11] F. Tuinstra and J. L. Koenig, J. Chem. Phys., 1970, 53(3), 1126.

[12] N. Larouche and B. L. Stansfield, Carbon, 2010, 48, 620.

[13] A. Cuesta, P. Dhamelincourt, J. Laureyns, A. Martinez-Alonso, and J. M. D. Tascon, Carbon, 1994, 32(8), 1523.

[14] M. A. Pimenta, G. Dresselhaus, M. S. Dresselhaus, L. G. Cancado, A. Jorio, and R. Saito, Phys. Chem. Chem. Phys., 2007, 9, 1276.

[15] K. W. R. Gilkes, S. Prawer, K. W. Nugent, J. Robertson, H. S. Sands, Y. Lifshitz, and X. Shi, J. Appl. Phys., 2000, 87, 7283.

[16] Z. Y. Chen, J. P. Zhao, T. Yano, T. Ooie, M. Yoneda, and J. Sakakibara, J. Appl. Phys., 2000, 88, 2305.

[17] F. Rasetti, Phys. Rev., 1929, 34, 367. 
[18] Y. Kawashima and G. Katagiri, Phys. Rev. B, 1995, 52, 10053.

[19] Z.- Y. Juang, C. -Y. Wu, C. -W. Lo, W. -Y. Chen, C.- F. Huang, J. -C. Hwang, F. -R. Chen, K. -C. Leou, and C. -H. Tsai, Carbon, 2009, 47, 2026.

[20] Y. Wang, D. C. Alsmeyer, and R. L. McCreery, Chem. Mater., 1990, 2, 557.

[21] W. Kaiser, P. H. Keck, and C. F. Lange, Phys. Rev., 1956, 101, 1264.

[22] W. G. Spitzer, D. A. Kleinman, and C. J. Frosch, Phys. Rev.,1959, 113, 133.

[23] T. G. Ros, A. J. van Dillen, J. W. Geus, and D. C. Koningsberger, ChemPhysChem, 2002, 3(2), 209

[24] A. Lee Smith, Spectrochimica Acta, 1960, 16, 87.

[25] J. M. O’Reilly and R. A. Mosher, Carbon, 1983, 21, 47.

[26] T. G. Ros, A. J. Van Dillen, J. W. Geus, and D. C. Koningsberger, Chem. Eur. J., $2002,8,2868$.

[27] K. Kwac and E. Geva, J. Phys. Chem. B, 2011, 115, 9184.

[28] J.-H. Zhou, Z. -J. Sui, J. Zhu, P. Li, D. Chen, Y. -C. Dai, and W.-K. Yuan, Carbon, 2007, 45, 785.

[29] Y. M. Ho, J. W. Liu, J. L. Qi, and W. T. Zheng, J. Phys, D: Appl. Phys., 2008, 41, 065308.

[30] R. N. Gayen and A. K. Pal, Appl. Surf. Sci., 2010, 256, 6172.

[31] H. -S. Kim, H. Lee, K. -S. Han, J.-H. Kim, M. -S. Song, M. -S. Park, J.-Y. Lee, and J. -K. Kang, J. Phys. Chem. B, 2005, 109(18), 8983. 


\section{Chapter 5}

\section{Evidence of wettability variation on carbon nanofiber layers grown on oxidized silicon substrates ${ }^{1}$}

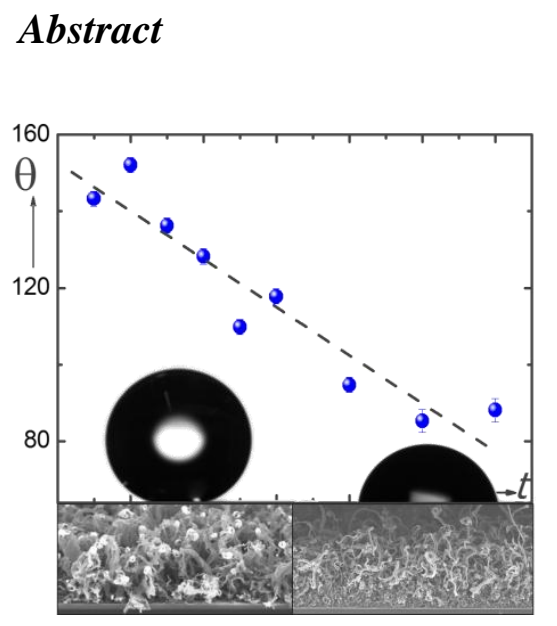

This chapter describes how layers of carbon nanofibers (CNFs) with controllable wettability can be synthesized by means of thermal catalytic chemical vapour deposition on nickel-based thin films on oxidized silicon supports. To achieve well-adhesive CNF-layers with a uniform surface coverage and tunable wettability without the necessity of post-synthesis treatments, a series of synthesis parameters is investigated: the pretreatment atmosphere (hydrogen or oxygen; 2 h, 500

$\left.{ }^{\circ} \mathrm{C}\right)$, the use of ethylene $\left(\mathrm{C}_{2} \mathrm{H}_{4}\right)$ or an ethylene/hydrogen $\left(\mathrm{C}_{2} \mathrm{H}_{4} / \mathrm{H}_{2}\right)$ mixture as hydrocarbon source, and the growth time (in the range 5-60 min). Fast and uniform CNF-growth is found on reduced Ni-based thin films using $\mathrm{C}_{2} \mathrm{H}_{4} / \mathrm{H}_{2}$ at a synthesis temperature of $635^{\circ} \mathrm{C}$. The $\mathrm{CNF}$ layers on Ni are superhydrophobic or highly hydrophobic for all growth times, but their adhesion to the support is poor for growth times $>30 \mathrm{~min}$. In contrast, the adhesion of CNFlayers on Ni/Ta is excellent. Moreover, the wettability of these as-synthesized CNF-layers can be controlled by variation of the growth time: from superhydrophobic ( $\leq 10 \mathrm{~min})$ to hydrophilic ( $\geq 50 \mathrm{~min})$. CNF-layers with such tunable wettability can be easily integrated in flow channels of silicon-based microfluidic systems, thereby offering numerous applications.

\footnotetext{
${ }^{1}$ This chapter is published as "Evidence of wettability variation on carbon nanofiber layers grown on oxidized silicon substrates" by H. Nair, R. M. Tiggelaar, D. B. Thakur, J. G. E. Gardeniers, A. van Houselt, L. Lefferts, Chemical Engineering Journal, 2013, 227, 56.
} 


\subsection{Introduction}

Surface wettability is of key importance in a myriad of applications. ${ }^{[1-7]}$ Especially hydrophobic surfaces are both technologically ${ }^{[4-7]}$ and fundamentally ${ }^{[8,9]}$ interesting. Superhydrophobic surfaces (contact angle $>150^{\circ}$ ) have been used to enhance mixing and drug delivery ${ }^{[2]}$. Other applications of hydrophobic layers are self-cleaning surfaces, ${ }^{[3]}$ anti-freezing surfaces, ${ }^{[4]}$ slip enhancement ${ }^{[5,6]}$ and their use for efficient catalytic multiphase microreaction technology. ${ }^{[7]}$ Wetting (hydrophilic) surfaces obey the no-slip boundary conditions, whereas non-wetting (hydrophobic) surfaces exhibit apparent slip of the order of microns. ${ }^{[8]}$

The aspect of reducing drag at the liquid-solid boundary is of importance for the efficiency of microfluidic systems. Mass transport in microfluidic devices, systems comprising enclosed flow channels with a high surface-to-volume ratio, is heavily influenced by viscous forces, that are mainly determined by the properties of the liquid-solid interface. When the solid has a water wetting surface, the linear velocity of the fluid at the interface is reduced to zero, resulting in a stagnant fluid layer, and consequently high energy dissipation. When the solid has a hydrophobic surface, the linear velocity of the fluid at the interface will be non-zero, which, in turn, will lower the energy dissipation due to drag.

Superhydrophobic surfaces (and to a less extent hydrophobic surfaces) combine a micro-structured surface with a low surface energy. ${ }^{[9]}$ In general, microstructures can be obtained by lithography, ${ }^{[10]}$ etching, electro-spinning, electrochemical reactions and sol-gel processing, ${ }^{[11]}$ particle deposition, ${ }^{[12]}$ or chemically grown carbonaceous nanostructures. ${ }^{[13]}$ These nanostructures can, based on their geometry, be classified as carbon nanotubes (CNTs) or CNFs. Single or multi-walled CNTs are formed by one or more concentrically rolled graphene sheet(s), thus forming mainly straight hollow cylinders. When the graphene sheets have an angle with respect to the main axis the resulting structures are CNFs. CNTs and CNFs can grow in two orientations with respect to their support: parallel aligned ('straight'), or randomly-oriented ('entangled'). ${ }^{[14]}$

In order to achieve superhydrophobicity, usually aligned CNTs (aCNTs) are chemically modified after synthesis, for example by coatings of fluoroalkylsilanes or 
thiols. ${ }^{[15-18]}$ In a few cases, superhydrophobicity of pristine aCNTs is reported, ${ }^{[19-22]}$ and for CNFs superhydrophobicity of as-grown structures is rarely reported. ${ }^{[23-25]}$

Goal of this work is to fabricate CNF-layers on Ni-based thin film coatings on oxidized silicon supports that have good adhesive properties, uniform surface coverage and tunable wettability without any post-synthesis treatment, and thus could be integrated in microfluidic devices. The method used is thermal catalytic chemical vapour deposition (TC-CVD), and a series of synthesis parameters is systematically varied. The TC-CVD growth settings are applied to different nickel configurations, i.e. $10 \mathrm{~nm} \mathrm{Ni}, 25 \mathrm{~nm} \mathrm{Ni}$, and $25 \mathrm{~nm} \mathrm{Ni} / 10 \mathrm{~nm}$ Ta. The parameters that are studied are the pretreatment atmosphere (hydrogen or oxygen; $2 \mathrm{~h}, 500{ }^{\circ} \mathrm{C}$ ), the use of ethylene $\left(\mathrm{C}_{2} \mathrm{H}_{4}\right)$ or ethylene/hydrogen $\left(\mathrm{C}_{2} \mathrm{H}_{4} / \mathrm{H}_{2}\right)$ as hydrocarbon source (synthesis temperature $635^{\circ} \mathrm{C}$ ), and the growth time (in the range 5-60 min). By varying the growth time on reduced $\mathrm{Ni} / \mathrm{Ta}$ on oxidized silicon, we show that the wettability of well-adhesive, assynthesized CNF-layers $\left(635{ }^{\circ} \mathrm{C}, \mathrm{C}_{2} \mathrm{H}_{4} / \mathrm{H}_{2}\right)$ can be tuned from superhydrophobic to hydrophilic.

\subsection{Experimental Methods}

\subsubsection{Preparation of nickel thin films on oxidized silicon substrates}

On silicon substrates ((100)-orientation, p-type, single-side polished, $100 \mathrm{~mm}$ diameter, $525 \pm 25 \mu \mathrm{m}$ thickness, resistivity 5-10 $\Omega . \mathrm{cm}$; Okmetic, Finland) a $250 \mathrm{~nm}$ thick $\mathrm{SiO}_{2}$ layer was grown via wet oxidation $\left(45 \mathrm{~min}, 1000{ }^{\circ} \mathrm{C}\right.$ ). By means of standard UV-lithography a pattern was defined in spin-coated photoresist (Olin, 90612), resulting in unmasked squares of $8 \mathrm{~mm} \times 8 \mathrm{~mm}$ onto which nickel-based thin films were deposited via electron-beam evaporation. Three different compositions were evaporated: $10 \mathrm{~nm}$ nickel (Ni), $25 \mathrm{~nm} \mathrm{Ni}$ and $25 \mathrm{~nm} \mathrm{Ni}$ on $10 \mathrm{~nm}$ tantalum (Ta). Post to metal deposition, an ultrasonic lift-off step in acetone ( $>20 \mathrm{~min}$; VLSI 100038, BASF) was performed, followed by further rinsing and spin drying. Finally, the nickel-coated substrates were diced into samples of $1 \mathrm{~cm} \times 1 \mathrm{~cm}$ (Disco DAD-321 dicing machine). Details of the sample fabrication procedure can be found elsewhere. ${ }^{[26]}$ 


\subsubsection{Synthesis of CNFs on Ni-coated samples}

Prior to CNF synthesis, the nickel-based thin film samples were ultrasonically cleaned in acetone (10 min, Branson 200 ultrasonic cleaner) and de-ionized water $\left(5 \mathrm{~min}, 25^{\circ} \mathrm{C}\right.$ ) to remove organic contaminants, followed by drying with pressurized technical air.

For CNF synthesis, samples were placed centrally on a flat quartz boat inside a quartz reactor and the temperature was increased in nitrogen $\left(\mathrm{N}_{2} ; 99.999 \%\right.$, INDUGAS $)$ to $500{ }^{\circ} \mathrm{C}\left(5 \mathrm{~K} \cdot \mathrm{min}^{-1}\right)$. At this temperature the pretreatment was done, in order to dewet the continuous as-evaporated thin film into nickel nanoparticles which are a requirement for catalytic CNF-growth. ${ }^{[26,27]}$ Two pretreatment environments were used: 20 vol.\% of hydrogen $\left(\mathrm{H}_{2} ; 99.999 \%\right.$, INDUGAS) in $\mathrm{N}_{2}$ and $20 \mathrm{vol} . \%$ of air in $\mathrm{N}_{2}$, both for $2 \mathrm{~h}$ at a total flow rate of $50 \mathrm{ml} \cdot \mathrm{min}^{-1}$. After this reduction or oxidation pretreatment, the temperature was increased in $\mathrm{N}_{2}\left(5 \mathrm{~K} \cdot \mathrm{min}^{-1}\right)$ to the desired CNFsynthesis temperature $\left(635^{\circ} \mathrm{C}\right)$. CNF-synthesis was performed by thermal catalytic chemical vapour deposition of 25 vol.\% ethylene $\left(\mathrm{C}_{2} \mathrm{H}_{4} ; 99.95 \%\right.$ PRAXAIR), with and without addition of $6.25 \mathrm{vol} . \% \mathrm{H}_{2}$, in $\mathrm{N}_{2}$ (total flow rate $100 \mathrm{ml} . \mathrm{min}^{-1}$ ) for various growth times. After the reaction time, the samples were cooled in $\mathrm{N}_{2}\left(10 \mathrm{~K} \cdot \mathrm{min}^{-1}\right)$ till room temperature. All flow rates are given under normal conditions (ambient temperature). Post to synthesis, the samples were characterized without additional functionalization of the CNFs.

Three synthesis parameters were varied in order to obtain the formation of well-adhesive CNFs with uniform coverage as well as tunable wettability on the surface of oxidized silicon samples with an evaporated nickel-based thin film: i) the pretreatment atmosphere (hydrogen or oxygen), ii) the use of ethylene or ethylene/hydrogen as hydrocarbon source (at $635^{\circ} \mathrm{C}$ ), and iii) the synthesis time (in the range 5-60 $\mathrm{min}$ ).

\subsubsection{Characterization}

The synthesized CNF-layers were investigated using high-resolution scanning electron microscopy (HR-SEM; LEO 1550) to determine the presence and morphology of CNFs as well as their uniformity in coverage (top view images). Cross-sectional SEM images were used to determine the thickness of the CNF-layers, 
and their adhesion to the silicon support. CNF-layer thicknesses were determined based on at least 3 representative images taken from different samples (exposed to identical processing). From each SEM-image 10 thickness measurements were determined using ImageJ software. The reported values are thus averages of $\geq 30$ measurements, and the standard deviation amounts up to $1.5 \mu \mathrm{m}$ for the thicker samples.

The adhesion of CNF-layers to the silicon support was also evaluated with fluid flows (air and water flows with a linear velocity of $100 \mathrm{~m} . \mathrm{s}^{-1}$ for $5 \mathrm{~min}$ and $2 \mathrm{~m} . \mathrm{s}^{-1}$ for $1 \mathrm{~h}$, respectively), in combination with weight measurements prior and post to these flow experiments (details in Ref. [26]).

Static contact angles were measured using an OCA contact angle meter (Data physics) using high purity milli-Q water (Millipore Synergy ${ }^{\circledR}$ system) at room temperature and a relative humidity of $\sim 33 \%$. A computer controlled Hamilton syringe $(500 \mu \mathrm{L})$ was used to inject a droplet of water $(3 \mu \mathrm{L})$. Droplets were placed on at least 5 different locations of each sample (i.e. samples covered with as-evaporated $\mathrm{Ni}$, pretreated $\mathrm{Ni}$, and CNF-layers), and the contact angle was determined from an elliptical fit through the droplet contour (side view imaging). The reported averaged values have a standard deviation of $\pm 1^{\circ}$.

\subsection{Results and Discussions}

\subsubsection{Influence of pretreatment atmosphere on CNF synthesis}

SEM images of CNFs obtained on oxidation-pretreated and reductionpretreated samples with $10 \mathrm{~nm}$ as-evaporated $\mathrm{Ni}$ after a reaction time of 1 hour with $\mathrm{C}_{2} \mathrm{H}_{4}$ at $635^{\circ} \mathrm{C}$ are shown in Figure 1. These synthesis settings yielded a layer with particles of carbon (or carbonaceous species) on the oxidation-pretreated sample (see Figure 1a), whereas on the reduction-pretreated sample CNFs (visible as wormlike features in Figure 1b) can be seen, which are equally distributed on the nickel coating.

In more detail, the $10 \mathrm{~nm} \mathrm{Ni}$ sample that was oxidation-pretreated only occasionally had fibers (approximately 1 fiber per square micrometer with a diameter of ca. $30 \mathrm{~nm}$ ), and this layer (consisting of carbon and fibers) had a poor adhesion to 
the surface of the sample, as evidenced by the occurrence of many cracks (inset of Figure 1a). The formation of carbon deposits on the oxidation-pretreated $10 \mathrm{~nm} \mathrm{Ni}$ coating is, in accordance with Ref.[28], attributed to catalyst deactivation (coke formation). In contrast, reduction-pretreated $10 \mathrm{~nm} \mathrm{Ni}$ samples were covered with many carbon fibers (diameter 30-50 nm). These CNFs were of tip-type growth with small nickel particles at the top of each fiber, and the CNFs showed an entangled morphology. The adhesion of this CNF-layer was rather poor (see inset Figure 1b: partial detachment of the CNF-layer). Clearly, the pretreatment conditions influence the dewetting of the $10 \mathrm{~nm}$ nickel film, and subsequent CNF nucleation and growth.
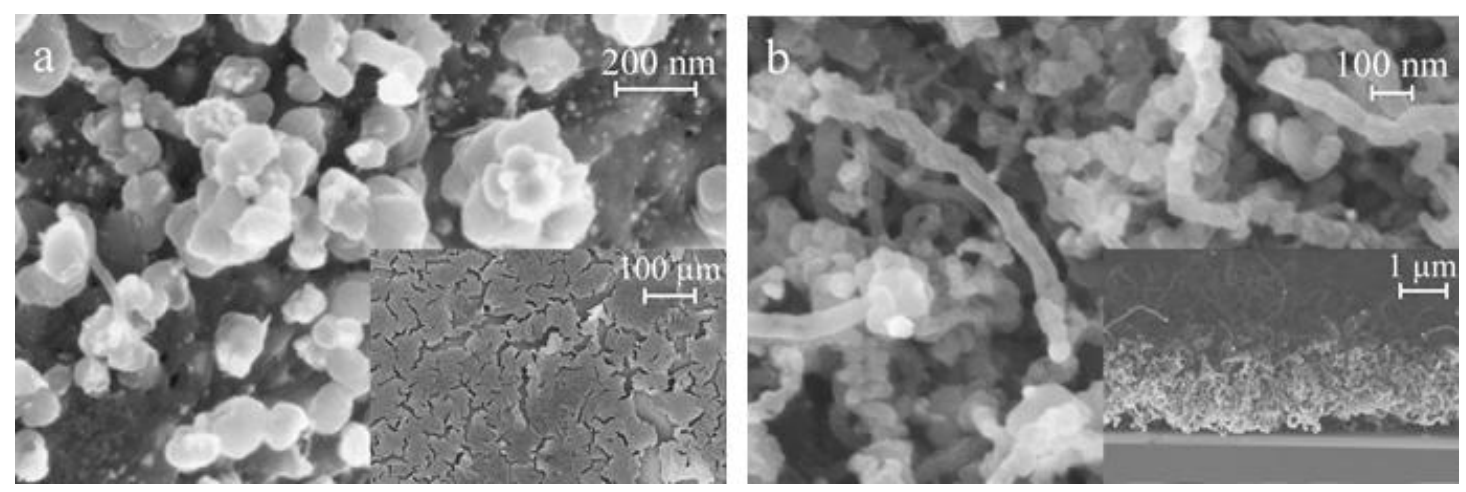

Figure 1: SEM-images of samples with $10 \mathrm{~nm}$ Ni after $1 \mathrm{~h}$ of $\mathrm{CNF}$ growth $\left(635{ }^{\circ} \mathrm{C}, \mathrm{C}_{2} \mathrm{H}_{4}\right)$. Panel (a) shows a top-view of an oxidation-pretreated sample with in the inset a larger field of view and panel (b) shows a top-view of a reduction-pretreated sample with a side-view in the inset.

SEM images of $10 \mathrm{~nm} \mathrm{Ni}$, as well as $25 \mathrm{~nm} \mathrm{Ni}$ and $25 \mathrm{~nm} \mathrm{Ni} / 10 \mathrm{~nm}$ Ta after oxidation and reduction are shown in Figure 2. The as-evaporated continuous Nilayers dewet into nanoparticles during the oxidation or reduction pretreatment. It can be seen that the pretreatment atmosphere, the thickness of the nickel layer and the use of a Ta adhesion layer influenced the dewetting of the $\mathrm{Ni}$ film (in terms of nanoparticle size and distribution, see Figure 2). The oxidation-pretreated samples appear to be less dewetted than their reduced counterparts (see Figures 2a, 2c, 2e vs. Figures 2b, 2d, 2f). Moreover, the thickness of $\mathrm{Ni}$ also influences the rate of dewetting, as can be seen from Figures $2 \mathbf{b}$ and $2 d(10 \mathrm{~nm} \mathrm{Ni}$ and $25 \mathrm{~nm} \mathrm{Ni}$; reduction-pretreated): thinner Ni-layers dewet faster. Clearly, the presence of a Ta adhesion layer favors the formation of nanoparticles (compare Figure 2e with $2 \mathbf{c}$ and Figure 2f with 2d). 

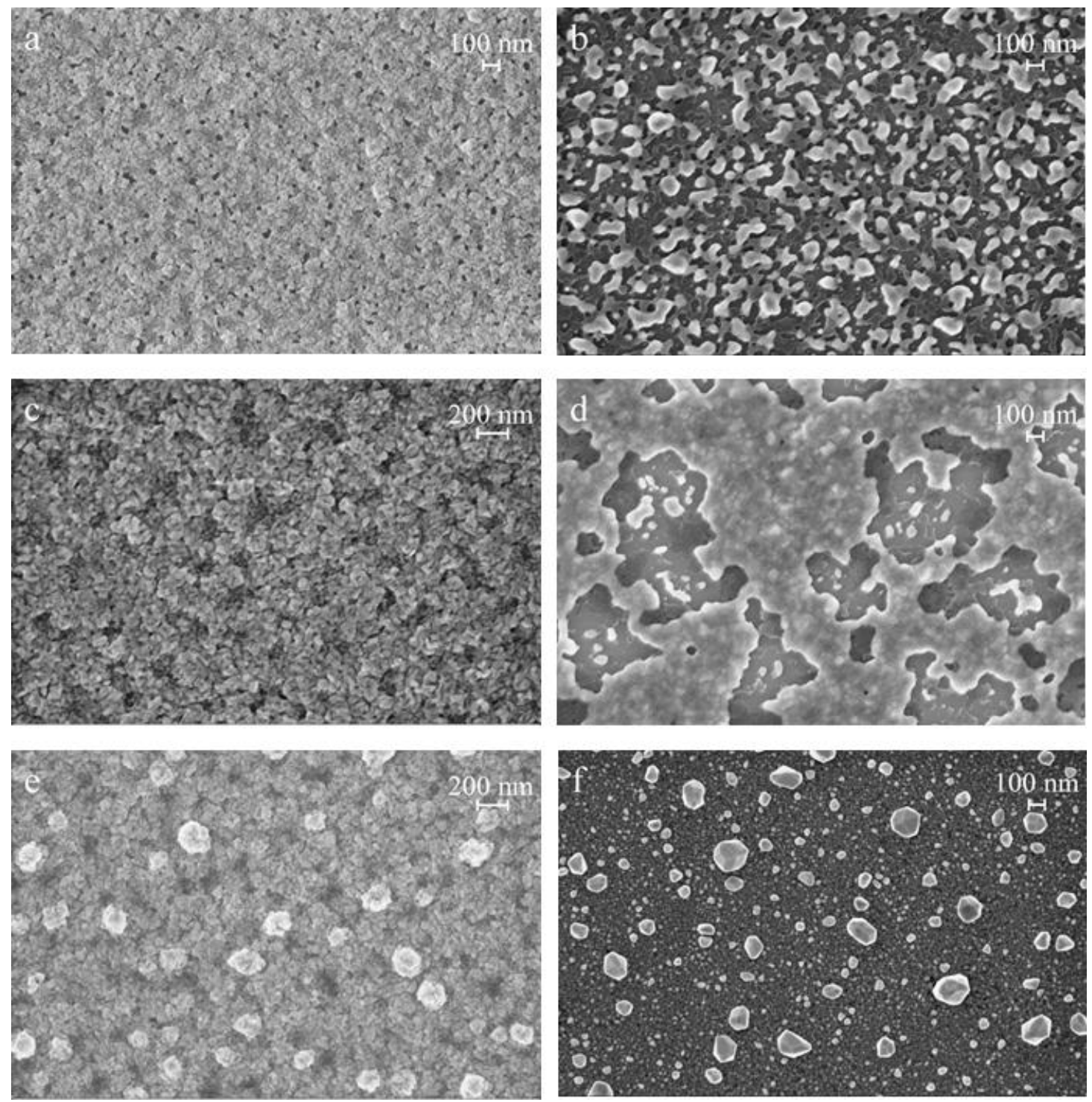

Figure 2: SEM-images of Ni-based thin films after oxidation or reduction pretreatment $(2 \mathrm{~h}, 500$ ${ }^{\circ} \mathrm{C}, \mathrm{O}_{2}$ or $\mathrm{H}_{2}$ ): (a) oxidized $10 \mathrm{~nm} \mathrm{Ni}$, (b) reduced $10 \mathrm{~nm} \mathrm{Ni}$, (c) oxidized $25 \mathrm{~nm} \mathrm{Ni}$, (d) reduced 25 nm Ni, (e) oxidized $25 \mathrm{~nm} \mathrm{Ni/10} \mathrm{nm} \mathrm{Ta} \mathrm{and} \mathrm{(f)} \mathrm{reduced} 25$ nm Ni/10 nm Ta.

For all thin films dewetting occurs via nucleation of holes or spinodal dewetting, followed by growth of nanoparticles. ${ }^{[29-31]}$ It is known that in an oxygen containing annealing atmosphere the interfacial tension between the support and nanoparticles is lower, ${ }^{[27]}$ due to formation of $\mathrm{NiO}$ at the surface of the nanoparticles. This decreases the surface mobility and the dewetting process of the nickel film. Under a reducing atmosphere, metals have a weaker interaction with oxidized substrates: hydrogen reduces the oxidized metal surface and, as a result the surface mobility increases, thereby fastening the dewetting process. Thus, for similar as- 
evaporated thin films dewetting is faster in a reducing atmosphere, as evidenced in Figure 2.

Reduced $10 \mathrm{~nm}$ Ni samples (Figure 2b) yielded smaller nanoparticles (with spherical or elliptical shape) with a higher areal density compared to $25 \mathrm{~nm}$ Ni film samples (Figure 2d), which is in agreement with our previous studies and literature. In fact, for the used reduction pretreatment conditions the $25 \mathrm{~nm} N i$ did not yet fully dewet into nanoparticles (Figure 2d), which is in agreement with other studies. ${ }^{[27]}$ The presence of $\mathrm{Ta}$ underneath the $\mathrm{Ni}$ thin film yielded numerous multifaceted nanoparticles: full dewetting of the $25 \mathrm{~nm} \mathrm{Ni}$ film (Figure 2f). This is because on tantalum the surface mobility of nickel is higher, and, hence, the rate of dewetting increases on Ni/Ta compared to a Ni film without an adhesion layer. ${ }^{[27]}$ The Ta layer can be observed as a continuous 'spongy' layer below the Ni particles (Figure 2f). Depending on the composition of the as-evaporated thin films and pretreatment conditions, the roughness of the as-evaporated films $\left(<2 \mathrm{~nm}^{[32]}\right)$ increases to $60-150$ nm after oxidation or reduction. ${ }^{[27]}$

Nickel nanoparticles are a requisite for CNF-synthesis. For samples with 10 $\mathrm{nm} \mathrm{Ni}, 25 \mathrm{~nm} \mathrm{Ni}$ and $25 \mathrm{~nm} \mathrm{Ni} / 10 \mathrm{~nm} \mathrm{Ta}$, these nanoparticles are only formed by means of a reduction pretreatment. Indeed, as evidenced in Figure 1, on oxidationpretreated Ni-based films nearly no CNF growth has occurred after $1 \mathrm{~h}$ synthesis $\left(\mathrm{C}_{2} \mathrm{H}_{4}\right.$ at $\left.635{ }^{\circ} \mathrm{C}\right)$, whereas reduction-pretreatment resulted in CNF-formation, independent of the composition of the nickel-based thin film. Cross-sectional SEM images of CNFs on reduced $25 \mathrm{~nm} \mathrm{Ni}$ and $25 \mathrm{~nm} \mathrm{Ni} / 10 \mathrm{~nm}$ Ta are shown in Figure 3 (see Figure 1b for $10 \mathrm{~nm} \mathrm{Ni).}$

CNF-layers synthesized on reduced $\mathrm{Ni} / \mathrm{Ta}$ have the highest $\mathrm{CNF}$ yield, i.e. a thicker CNF-layer, and excellent adhesion to the silicon support material. In case of a $25 \mathrm{~nm}$ Ni film without Ta, the weaker adhesion results in the removal of CNFs during sample preparation for the cross-sectional SEM image in Figure 3b. This is in agreement with previous work on fused silica substrates, which are - from the pointof-view of the composition of the surface on which the Ni-based films are deposited comparable to oxidized silicon. ${ }^{[26]}$ Exposure to fluid flows revealed that CNF-layers synthesized on $\mathrm{Ni} / \mathrm{Ta}$ had excellent adhesion (weight loss of the CNF-layers below 
6\%), whereas CNF-layers on $\mathrm{Ni}$ were (nearly) completely flushed away from the support material, indicating poor adhesion. The use of a Ta layer ensures an excellent adhesion of the synthesized CNF-layers to the support, whereas the roughness of the Ni films after pretreatment has no noticeable influence on the adhesive quality. Indeed, droplet impact also revealed a difference in adhesion of CNF-layers on $\mathrm{Ni} / \mathrm{Ta}$ and Ni films (see section 5.3.3.).
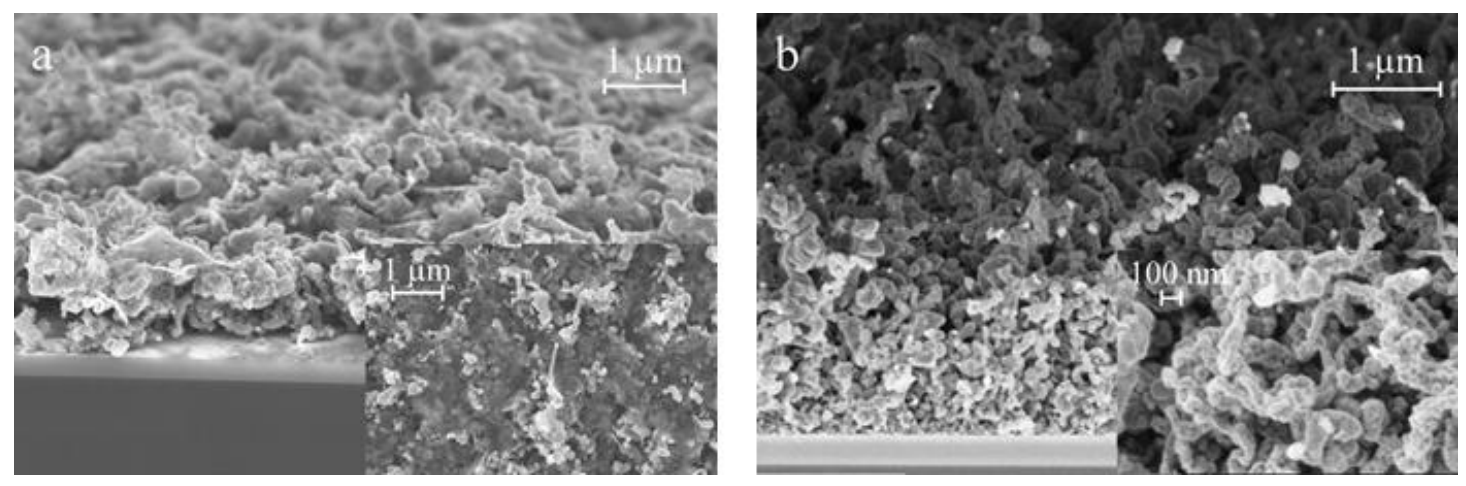

Figure 3: Cross-sectional SEM-images of CNFs grown $\left(1 \mathrm{~h}, 635^{\circ} \mathrm{C}, \mathrm{C}_{2} \mathrm{H}_{4}\right)$ on (a) reduced $25 \mathrm{~nm}$ $\mathrm{Ni}$, and (b) reduced $25 \mathrm{~nm} \mathrm{Ni/10} \mathrm{nm}$ Ta. The insets show top-view images.

\subsubsection{Influence of hydrogen on CNF synthesis}

In Figure 4, SEM images are shown of CNFs synthesized with a reactant mixture of $\mathrm{C}_{2} \mathrm{H}_{4}$ and $\mathrm{H}_{2}\left(1 \mathrm{~h}, 635{ }^{\circ} \mathrm{C}\right)$ on $10 \mathrm{~nm} \mathrm{Ni}$ samples exposed to various pretreatment conditions. Addition of hydrogen to ethylene enhances the growth of carbon nanofibers, and yields uniform coverage of samples with CNFs. The thickness of the CNF-layer has increased with respect to results obtained without $\mathrm{H}_{2}$ (Figure 1). This is in agreement with literature: the addition of hydrogen increases the CNF formation rate, resulting in the formation of long, thin fibers by slowing down the catalyst deactivation. ${ }^{[33,34]}$ It is discussed by Chinthaginjala et. al. ${ }^{[34]}$ that the addition of $\mathrm{H}_{2}$ influences the formation of a CNF layer via 2 effects: 1) $\mathrm{H}_{2}$ addition decreases the CNF growth rate on active Ni particles, 2) $\mathrm{H}_{2}$ delays deactivation of nanoparticles (i.e. prevents encapsulation of $\mathrm{Ni}$ ) which might occur due to excess deposition of carbon via decomposition of ethylene, thus leading to thicker CNF layers and hence to a higher CNF formation rate. 

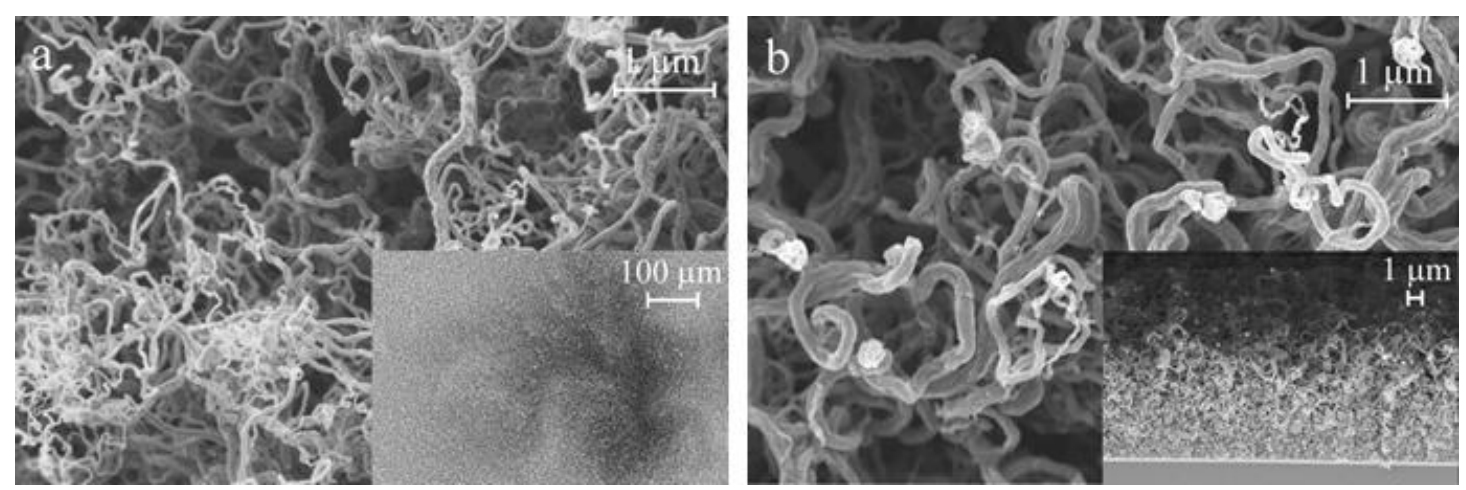

Figure 4: Top-view SEM-images of CNFs grown $\left(1 \mathrm{~h}, 635{ }^{\circ} \mathrm{C}, \mathrm{C}_{2} \mathrm{H}_{4} / \mathrm{H}_{2}\right)$ on $10 \mathrm{~nm} \mathrm{Ni}$ after (a) oxidation pretreatment (the inset shows an image with a lower magnification) and (b) reduction pretreatment (the inset shows a cross-sectional image).

In fact, even without pretreatment, CNFs were formed on $10 \mathrm{~nm} \mathrm{Ni}$ with good surface uniformity. However, these fibers were very weakly attached (based on crosssectional SEM images, not shown). Presumably, addition of hydrogen to ethylene 'induces' the formation of nickel nanoparticles during the first minutes of the CNFsynthesis step, and thus CNF-formation, despite the absence of any thermal pretreatment of the Ni film prior to CNF-synthesis.

CNF-layers formed with an ethylene-hydrogen mixture on oxidationpretreated $10 \mathrm{~nm} \mathrm{Ni}$ had a 'wavy appearance' (inset Figure 4a) due to local detachment of the CNF-layer (poor adhesion), and the CNF-diameters were in the range 50-100 $\mathrm{nm}$. In the case of hydrogen assisted growth on reduction-pretreated $\mathrm{Ni}$ (Figure 4b), the adhesion of the CNF-layers was better (less detachment), and the CNF-diameters were larger (100-200 nm). The amount of fibers in Figure $4 \mathbf{b}$ (reduction pretreated) appears slightly lower than the amount of fibers in Figure 4a (oxidation pretreated). For samples containing $25 \mathrm{~nm} \mathrm{Ni}$, similar results were found in terms of growth, adhesion and formation rate of CNFs. In the case of oxidationpretreatment, thin smooth CNFs (diameter: 50-100 nm) with an apparent high density were formed on $\mathrm{Ni}$ and $\mathrm{Ni} / \mathrm{Ta}$, while for reduction-pretreatment, thicker CNFs with a (slightly) lower density were observed (diameter $\sim 100 \mathrm{~nm}$ on $\mathrm{Ni}$, and $\sim 200 \mathrm{~nm}$ on $\mathrm{Ni} / \mathrm{Ta}$, respectively). Moreover, $\mathrm{Ni} / \mathrm{Ta}$ samples contained a broader range of $\mathrm{CNF}$ diameters, due to the larger variation in the size of nickel nanoparticles during pretreatment (see Figures 2b, 2d, 2f). CNFs synthesized on Ni/Ta had excellent 
adhesive properties, significantly better than CNFs on $10 \mathrm{~nm}$ or $25 \mathrm{~nm}$ Ni-films, independent of the pretreatment conditions.

In conclusion, CNF-synthesis on reduced $\mathrm{Ni} / \mathrm{Ta}$ thin film samples using $\mathrm{C}_{2} \mathrm{H}_{4} / \mathrm{H}_{2}$ results in CNF-layers with excellent adhesion to the support and uniform coverage of the surface. With this hydrocarbon source on reduced Ni thin films without Ta CNF-layers can be grown uniformly as well, but these layers suffer from poor adhesion.

\subsubsection{Wettability of synthesized CNF-layers}

The wettability of CNF-layers after $1 \mathrm{~h}$ of synthesis grown on reductionpretreated $\mathrm{Ni}$ and $\mathrm{Ni} / \mathrm{Ta}$ samples (synthesis conditions: $635{ }^{\circ} \mathrm{C}, \mathrm{C}_{2} \mathrm{H}_{4} / \mathrm{H}_{2}$ ) was determined. CNF-layers on $10 \mathrm{~nm}$ Ni samples were hydrophobic: contact angles were ca. $138^{\circ}$, whereas for $25 \mathrm{~nm} \mathrm{Ni}$, no contact angle (CA) could be determined due to detachment of the CNF-layer. In contrast, CNF-layers on $\mathrm{Ni} / \mathrm{Ta}$ exhibit CA-values of $\sim 88^{\circ}$. However, although hydrophobic, CNF-layers on Ni-samples had an important disadvantage: the attachment of such CNFs to the oxidized silicon substrate was poor. This was experienced during static CA-measurements: the impact of droplets resulted in partial removal/detachment of the CNF-layers.

It is known that the morphological properties like thickness and porosity of the CNF-layers may influence the wetting behavior. ${ }^{[10,11]}$ In an attempt to get more insight in the influence of the morphological changes during synthesis, the synthesis time of the CNF-layers was varied for reduced nickel-based samples. CNF-layers were grown on reduced $\mathrm{Ni}$ and $\mathrm{Ni} / \mathrm{Ta}$ for various growth times in the range 5-60 min $\left(635{ }^{\circ} \mathrm{C}, \mathrm{C}_{2} \mathrm{H}_{4} / \mathrm{H}_{2}\right)$, and the measured thicknesses (as deduced from cross-sectional SEM images) of the CNF-layers are plotted in Figure 5a.

In case of samples with $\mathrm{Ni}$ only, the CNF-layer thickness increased with time up to $30 \mathrm{~min}$ of growth, and the thickness levelled afterwards $(10 \mathrm{~nm} \mathrm{Ni})$ or even decreased slightly (25 nm Ni). The CNF-layer thickness on Ni/Ta increased up to 25 min of growth, followed by a plateau around $35 \mu \mathrm{m}$ for longer synthesis times: this is due to deactivation of catalyst particles. Similar observations were reported for CNFgrowth on nickel foams. ${ }^{[28]}$ 

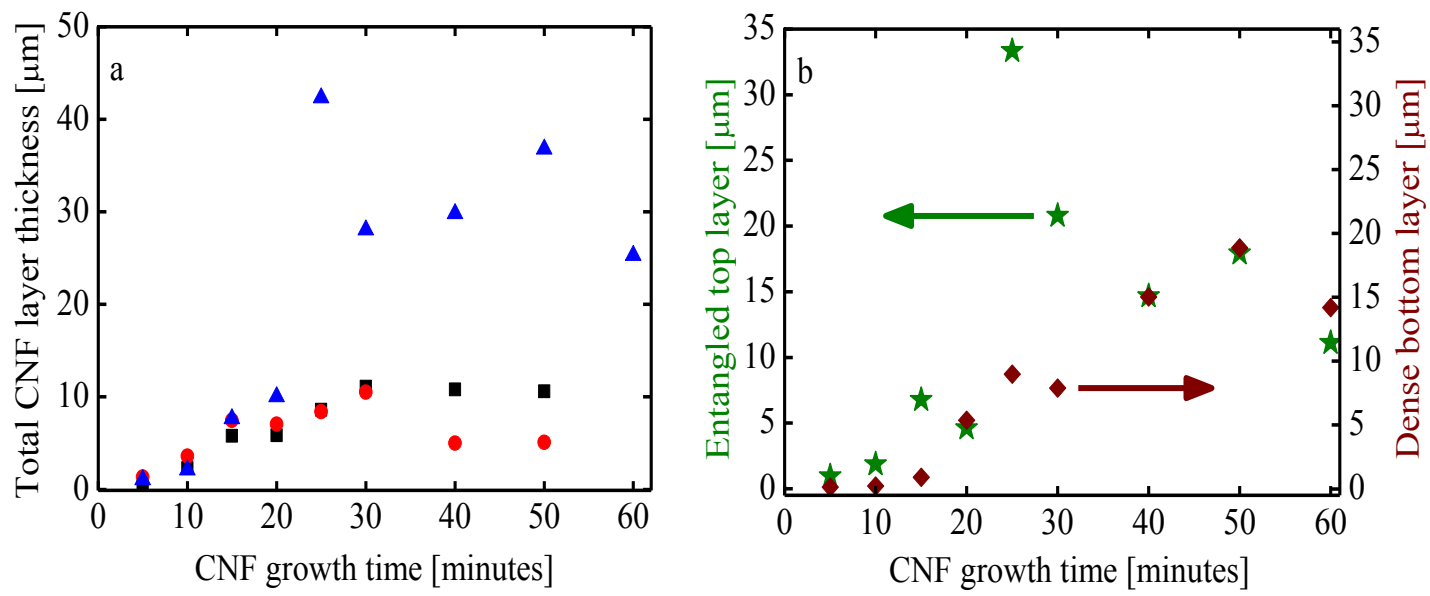

Figure 5: (a) Total CNF-layer thicknesses as a function of growth time after CNF synthesis (635 ${ }^{\circ} \mathrm{C}, \mathrm{C}_{2} \mathrm{H}_{4} / \mathrm{H}_{2}$ ) on reduction-pretreated $25 \mathrm{~nm} \mathrm{Ni} / 10 \mathrm{~nm}$ Ta ( $(\Delta), 25 \mathrm{~nm} \mathrm{Ni}(\bullet)$ and $10 \mathrm{~nm} \mathrm{Ni} \mathrm{( \bullet ).}$ (b) Thicknesses of entangled fiber-part at the top ( $\star$ ) and the dense layer at the bottom $(\diamond)$ as a function of growth time for CNF-layers on $\mathrm{Ni} / \mathrm{Ta}\left(635^{\circ} \mathrm{C}, \mathrm{C}_{2} \mathrm{H}_{4} / \mathrm{H}_{2}\right)$.

In addition, careful inspection of the SEM images of the grown CNF-layers on $\mathrm{Ni} / \mathrm{Ta}$ (Figure 6) reveals that for growth times of 20-30 min the CNF-layers are more (vertically) aligned, and that for longer synthesis times ( $\geq 30 \mathrm{~min}$ ) the CNFs start to curl and intertwine progressively with growth time (which shows up as a reduction in the height of the entangled top layer in Figure 5b), leading to a densification of the CNF-layer. These effects might contribute to a reduction $(25 \mathrm{~nm} \mathrm{Ni})$ or levelling $(\mathrm{Ni} / \mathrm{Ta})$ of the total layer thickness for growth times above $30 \mathrm{~min}$ (Figure 5a). In addition, small variation in the growth temperature (from run-to-run) might influence the growth kinetics of the CNFs substantially, giving rise to the observed variations in total layer thickness.

CNF-layers on Ni/Ta had excellent adhesion to the support, whereas CNFlayers on $\mathrm{Ni}$ were prone to peeling off/detachment for growth times $>30 \mathrm{~min}$ (the longer the growth time, the worse the attachment of CNFs on Ni samples). A growth time of $\leq 15 \mathrm{~min}$ is the best for fairly good adhesion of CNF-layers on reduced Ni.

Figure 6 shows the typical SEM images of CNF-layers synthesized on reduced $\mathrm{Ni} / \mathrm{Ta}$ for various growth times. 

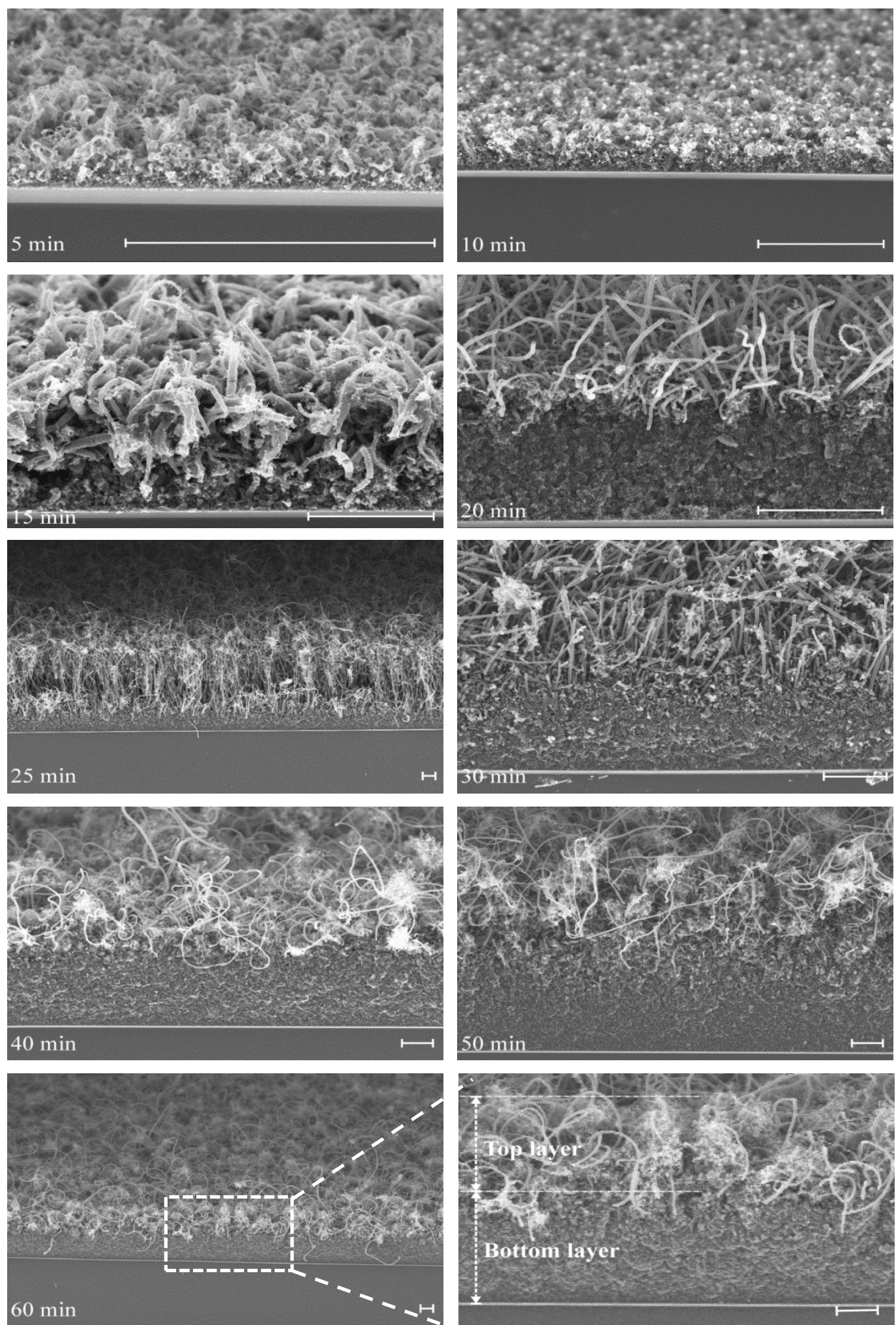

Figure 6: Cross-sectional SEM-images of CNF-layers synthesized $\left(635{ }^{\circ} \mathrm{C}, \mathrm{C}_{2} \mathrm{H}_{4} / \mathrm{H}_{2}\right)$ on reduced $\mathrm{Ni} / \mathrm{Ta}$ for various growth times. The scale bar corresponds to $5 \mu \mathrm{m}$ in each image. The top and bottom layers are indicated in the image of the sample grown in $60 \mathrm{~min}$. 
For longer growth-times ( $>15 \mathrm{~min})$ a "densified" C-layer is recognizable in which no individual fibers can be discriminated (this C-layer is most likely composed of amorphous carbon ${ }^{[35]}$ ), with a layer on its top, containing clearly observable, randomly-oriented, entangled CNFs. The bottom layer ("dense" C-layer) and top layer (entangled CNFs) are indicated in the cross sectional SEM images for the sample after 60 min growth in Figure 6. The adhesion of the CNF layer to the substrate is excellent on these substrates, which is most probably originating from the dense Clayer, which anchors the CNFs firmly to the substrate. The thicknesses of both the entangled fiber-part as well as the dense C-part as a function of growth time are given in Figure 5b. The thickness of the dense carbon sublayer increases approximately linearly with the growth time, which is not the case for the sublayer of entangled fibers: For growth times above $25 \mathrm{~min}$, the height of the entangled CNF layer decreases to around $18 \mu \mathrm{m}$.

In. Figure 7, characteristic photographs are shown of a droplet on CNF-layers that are superhydrophobic or hydrophilic, as-synthesized on reduced $\mathrm{Ni} / \mathrm{Ta}$ using two different growth times (10 and $50 \mathrm{~min}$ ).
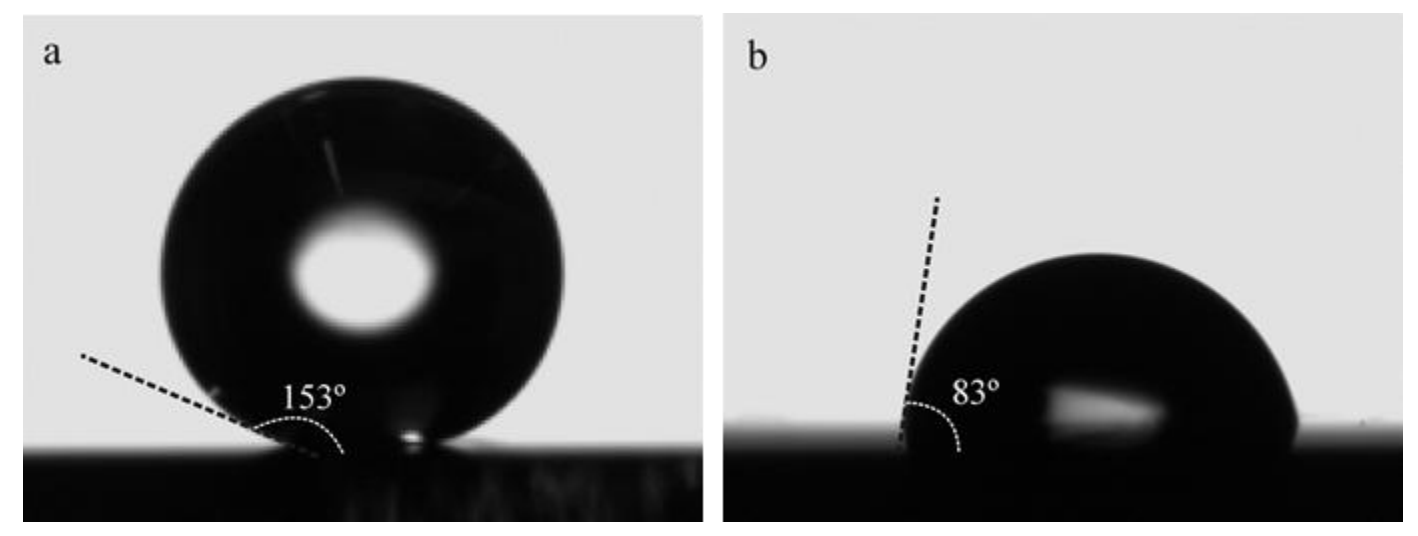

Figure 7: Photographs of water droplets on as-grown $\left(635^{\circ} \mathrm{C}, \mathrm{C}_{2} \mathrm{H}_{4} / \mathrm{H}_{2}\right) \mathrm{CNF}$-layers. After 10 min growth the layer is superhydrophobic (a), while after 50 min growth the layer is hydrophilic (b).

The CA-values extracted from these photographs are shown in Figure $\mathbf{8}$ as a function of the growth time. For comparison we determined the CA-values of the as evaporated and pretreated substrates prior to CNF growth (see Table 1). Except for the as evaporated $10 \mathrm{~nm}$ Ni substrates, all the contact angles prior to CNF growth are very comparable $\left(94.5^{\circ} \pm 1.5^{\circ}\right)$. The somewhat lower contact angle for the as 
evaporated $10 \mathrm{~nm}$ Ni substrates might arise from a partial coverage of the Si surface, hence showing an averaged contact angle between the contact angles on $\mathrm{Ni}$ and $\mathrm{Si}$.
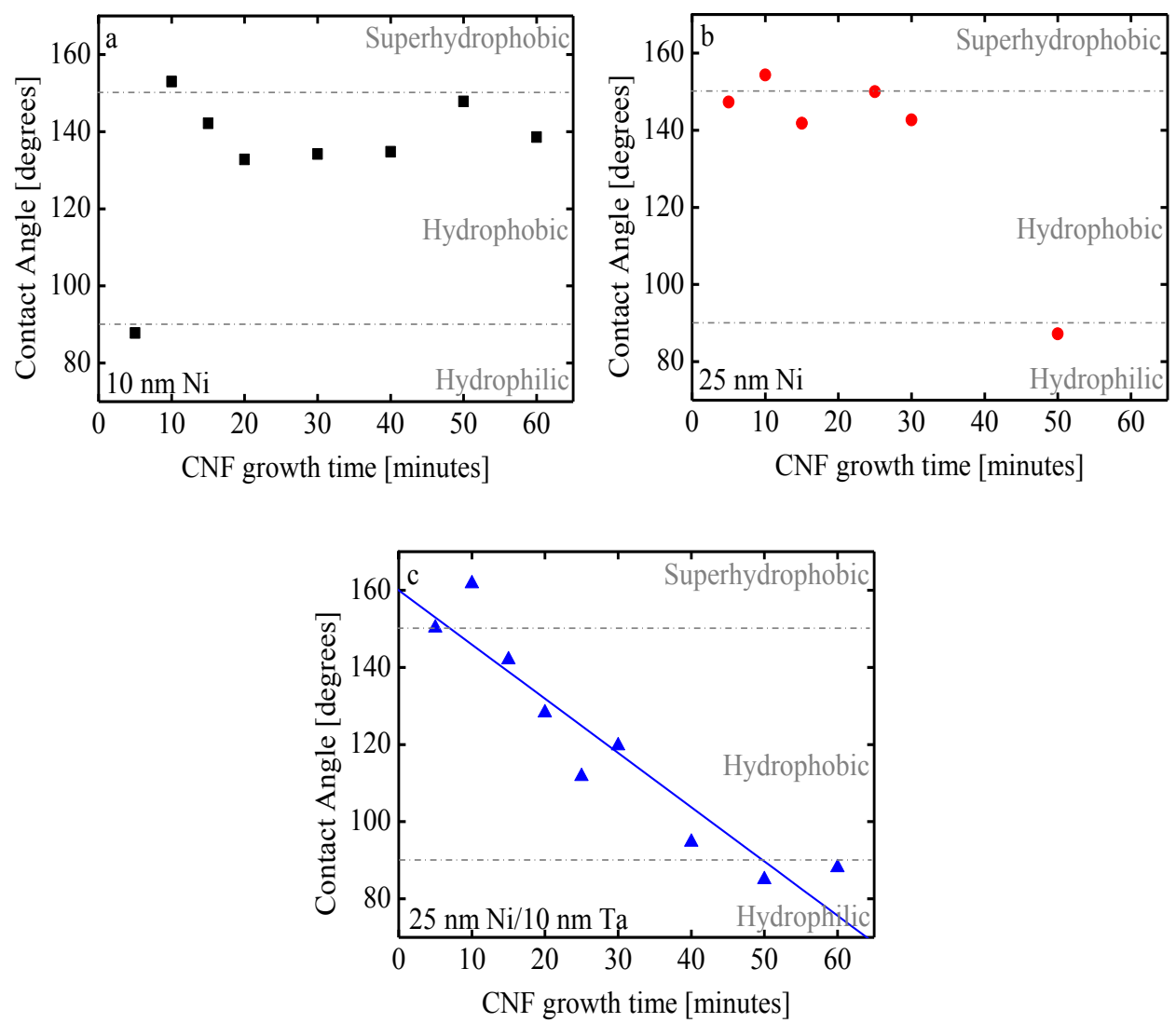

Figure 8: Contact angle values measured on CNF-layers as a function of growth time after CNF synthesis $\left(635{ }^{\circ} \mathrm{C}, \mathrm{C}_{2} \mathrm{H}_{4} / \mathrm{H}_{2}\right)$ on reduced (a) $10 \mathrm{~nm} \mathrm{Ni}(\bullet)$, (b) $25 \mathrm{~nm} \mathrm{Ni}(\bullet)$ and (c) $25 \mathrm{~nm} \mathrm{Ni} / 10$ $\mathrm{nm} \mathrm{Ta}(\Delta)$. In (c) a linear trendline is added as a guide to the eyes.

Table 1: Contact angle values of the substrates prior to CNF growth.

\begin{tabular}{|c|c|c|}
\hline Substrate & Treatment & Contact angle $\left({ }^{\circ}\right)$ \\
\hline \multirow{3}{*}{$10 \mathrm{~nm} \mathrm{Ni}$} & As evaporated & 78 \\
\hline & Reduced $\left(2 \mathrm{~h}, 500^{\circ} \mathrm{C}\right)$ & 96 \\
\hline & Oxidized $\left(2 \mathrm{~h}, 500^{\circ} \mathrm{C}\right)$ & 95 \\
\hline \multirow{3}{*}{$25 \mathrm{~nm} \mathrm{Ni}$} & As evaporated & 96 \\
\hline & Reduced $\left(2 \mathrm{~h}, 500^{\circ} \mathrm{C}\right)$ & 94 \\
\hline & Oxidized $\left(2 \mathrm{~h}, 500^{\circ} \mathrm{C}\right)$ & 95 \\
\hline \multirow{3}{*}{$25 \mathrm{~nm} \mathrm{Ni} / 10 \mathrm{~nm} \mathrm{Ta}$} & As evaporated & 96 \\
\hline & Reduced $\left(2 \mathrm{~h}, 500^{\circ} \mathrm{C}\right)$ & 93 \\
\hline & Oxidized $\left(2 \mathrm{~h}, 500^{\circ} \mathrm{C}\right)$ & 93 \\
\hline
\end{tabular}


As a result of the CNF-growth the CAs change. It can be observed in Figure 8 that CNF-layers are superhydrophobic for a growth time of 10 min on samples with a "thick" Ni-content (25 nm as-evaporated). More generally, if no Ta is used underneath the $\mathrm{Ni}$, almost all growth times yielded highly hydrophobic CNF-layers with a CA above $130^{\circ}$. It is believed that these high CA-values were maintained because the thickness of the CNF-layer was not increasing significantly for growth times $\geq 20$ min, most likely due to deactivation of the catalytic nickel nanoparticles, which stopped the formation of carbon fibers. However, for very long synthesis times, the adhesion of these very hydrophobic CNFs to the silicon support became very poor. In contrast, CNF-layers on $\mathrm{Ni} / \mathrm{Ta}$ showed their excellent adhesion for all growth times, but the wettability of these CNF-layers gradually decreased towards hydrophilic. Thus, only thin CNF-layers - with a thickness of 2-3 $\mu \mathrm{m}$ - that are grown for $10 \mathrm{~min}$ on $\mathrm{Ni} / \mathrm{Ta}$ or $\mathrm{Ni}$ are superhydrophobic with good adhesion to the support.

Next we discuss the decreasing contact angle with growth time, observed for the stable CNF-layers grown on $\mathrm{Ni} / \mathrm{Ta}$ (see Figure 8c). Superhydrophobicity is usually explained by either the Wenzel model, when the liquid wets the surface completely, or by the Cassie-Baxter model when the liquid wets only the top of the surface asperities. ${ }^{[9]}$ Assuming homogeneous chemical properties of the surface, the presence of nanostructures amplifies the wettability of the corresponding flat surfaces in the Wenzel case, since the actual wetted area increases, while in the Cassie-Baxter case, the presence of nanostructures will always result in higher contact angles compared to the corresponding flat surface. In both cases the geometric properties of the surface (in our case the diameter, length, orientation and density of the fibers in the entangled layer) will influence the actually wetted area. Top-view and crosssectional SEM-images (e.g. Figure 6) reveal a similar appearance of the top-part of all CNF-layers, i.e. entangled fibers with diameter in the range 50-200 nm, with rather identical void spaces (i.e. no large fluctuations in the surface porosity). The decreasing contact angle with increasing growth time can therefore not be related to a 'simple' (i.e. only based on geometric considerations) Cassie-Baxter state, for which one would need a decreasing surface porosity (and hence roughness) with increasing growth time to reproduce the observed trend. For a Wenzel picture, we keep in mind that during growth, the actual surface per unit flat area increases monotonically. In the 
Wenzel picture, a decreasing trend, as observed in our data with increasing growth time is associated with a hydrophilic surface and would then start initially with at most a contact angle of $90^{\circ}$. Therefore a 'simple' Wenzel picture is also insufficient to explain the observed trends.

The presence of Ta might change the evolution of the catalytically grown CNF with time compared to the substrates without Ta. One could for instance image that Ta nanoparticles induce a change in the growth mode of the CNFs from initially base growth (see the SEM image after $5 \mathrm{~min}$ in Figure 6) to more tip growth in the later growth (see the SEM images after 10 and $20 \mathrm{~min}$ ). Such a change in growth mode is at this moment however, speculation and one would need dedicated in situ microscopy to verify this hypothesis.

The surface roughness and chemistry of the CNFs may also change during the growth. In Figure 9 we show representative SEM images of individual CNFs grown on reduced $\mathrm{Ni} / \mathrm{Ta}\left(1 \mathrm{~h}, 635{ }^{\circ} \mathrm{C}, \mathrm{C}_{2} \mathrm{H}_{4} / \mathrm{H}_{2}\right)$. It is noticeable that with increasing growth time, the surface roughness of the thicker CNFs decreases, while their diameter increases slightly. These morphological and chemical changes will clearly influence the wetting properties of the CNF surfaces, but further research is needed to investigate and model their exact role.

The apparently decisive role of the CNF-layer thickness in the wettability is also reported in earlier work in our group, ${ }^{[25]}$ where, in contrast to our findings here, an increasing contact angle with increasing layer thickness was reported for CNFlayers grown on Ni metal foils. For the CNF-layers grown on polycrystalline Ni foils the surface roughness was reported on the scale of tens of microns, ${ }^{[25]}$ while roughness in such dimensions is completely absent on the CNF-layers grown on $\mathrm{Ni}$ thin films in this study. In addition, for the CNF-layers grown on Ni foils it was assumed that the surface chemistry of the CNF did not have a noticeable effect on the wettability of the CNF-layers, ${ }^{[25]}$ while in the Ni thin film based CNF layers in this study a decisive role of changes in the surface chemistry cannot be ruled out. These differences in the CNF-layer properties on Ni foils versus $\mathrm{Ni}$ thin films might very well be related to the different wetting properties on these layers.

The stability of the superhydrophobicity of the CNF-layers on reduced $\mathrm{Ni} / \mathrm{Ta}$ 
was verified by repetitive measurements of the contact angle on the same sample for a period of 36 months. During this period we observed -in contrast to observations on pristine superhydrophobic CNT-samples ${ }^{[36]}$ - no influence of UV irradiation or dark storage on the wettability of the CNF-layers. We did not observe any change in the contact angle over this period. In addition, the contact line of evaporating droplets is strongly pinned on CNF substrates (hysteresis values up to $65^{\circ}$ were measured), which, together with the high contact angle, makes these substrates ideally suited for evaporation studies in the constant contact area mode. ${ }^{[23]}$

In summary, on pure-Ni samples (super)hydrophobic CNF-layers can be obtained, however, their attachment to the Si substrate is rather poor, which limits their practical use. For CNF-layers grown on Ni/Ta substrates the adhesion to the $\mathrm{Si}$ substrate is excellent and their wettability can be effectively tuned from superhydrophobic to hydrophilic by variation of the growth time.
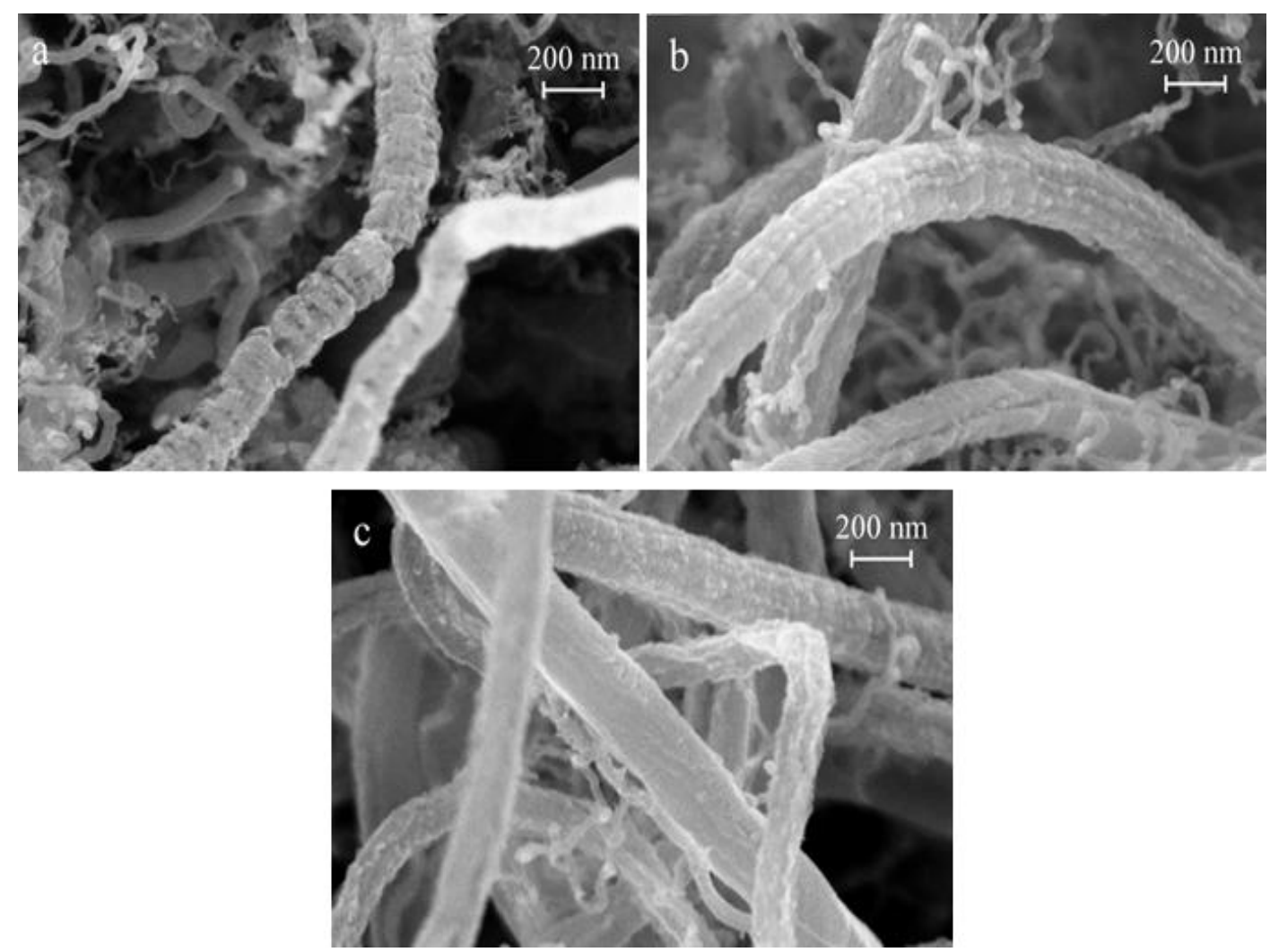

Figure 9: SEM-images of CNFs synthesized on reduced Ni/Ta $\left(1 \mathrm{~h}, 635{ }^{\circ} \mathrm{C}, \mathrm{C}_{2} \mathrm{H}_{4} / \mathrm{H}_{2}\right)$ for various growth times: a) $15 \mathrm{~min}$, b) $40 \mathrm{~min}$, and c) $50 \mathrm{~min}$. 


\subsection{Conclusions and Outlook}

Various nickel-based thin films - $10 \mathrm{~nm} \mathrm{Ni,} 25 \mathrm{~nm} \mathrm{Ni}$ and $25 \mathrm{~nm} \mathrm{Ni} / 10 \mathrm{~nm} \mathrm{Ta}$ - on oxidized silicon supports were exposed to an oxidation or reduction pretreatment $\left(2 \mathrm{~h}, 500{ }^{\circ} \mathrm{C}\right)$ in order to dewet these continuous films into nanoparticles. $\mathrm{Ni}$ nanoparticle formation, which is required for CNF-growth, was only observed when a reductive pretreatment was applied. Ni nanoparticle size and distribution depended on the thickness of the nickel film as well as the presence of a tantalum adhesion layer. The nanoparticle size distribution influenced the diameter of the CNFs qualitatively.

On reduced $\mathrm{Ni}$ and $\mathrm{Ni} / \mathrm{Ta}$ thin films CNFs could be TC-CVD synthesized (with uniform surface coverage) using ethylene, and addition of hydrogen enhanced the formation rate of CNF-layers. Whereas the adhesion of CNF-layers on Ni samples was poor (in particular for growth times longer than $30 \mathrm{~min}$ ), on $\mathrm{Ni} / \mathrm{Ta}$ the adhesion of CNF-layers was excellent.

Wettability investigations on CNF-layers grown on reduction-pretreated $\mathrm{Ni}$ and $\mathrm{Ni} / \mathrm{Ta}$ (synthesis conditions: $635^{\circ} \mathrm{C}, \mathrm{C}_{2} \mathrm{H}_{4} / \mathrm{H}_{2}$ ) revealed that $\mathrm{CNF}$ layers grown on Ni were (nearly) superhydrophobic for all growth times, whereas the wettability of CNF-layers on $\mathrm{Ni} / \mathrm{Ta}$ changed gradually from superhydrophobic for growth times $\leq$ $10 \mathrm{~min}$ to hydrophilic for growth times $\geq 50 \mathrm{~min}$.

The possibility to tune the wettability of well-adhesive, as-synthesized CNFlayers - no post-synthesis treatments were applied - enables the systematic variation of the wettability in flow channels of silicon-based microfluidic systems by a CNF coating. For example, hydrophilic CNF-layers can be used as catalyst support in microreactors $^{[37,38]}$ and superhydrophobic CNFs as coatings for drag reduction (superlubrification).

\subsection{References}

[1] X. J. Feng and L. Jiang, Adv. Mater., 2006, 18, 3063.

[2] S. T. Yohe, Y. L. Colson, and M. W. Grinstaff, J. Am. Chem. Soc., 2012, 134, 2016.

[3] R. Blossey, Nat. Mater., 2003, 2, 301. 
[4] A. J. Meuler, G. H. McKinley, and R. E. Cohen, ACS Nano, 2010, 4, 7048.

[5] G. McHale, M. I. Newton, and N. J. Shirtcliffe, Soft Matter, 2010, 6, 714.

[6] P. Joseph, C. Cottin-Bizonne, J.-M. Benoît, C. Ybert, C. Journet, P. Tabeling, and L. Bocquet, Phys. Rev. Lett., 2006, 97, 156104.

[7] H. C. Aran, J. K. Chinthaginjala, R. Groote, T. Roelofs, L. Lefferts, M. Wessling, and R.G.H. Lammertink, Chem. Eng. J., 2011, 169, 239.

[8] L. Bocquet and E. Charlaix, Chem. Soc. Rev., 2010, 39, 1073.

[9] D. Quéré, Annu. Rev. Mater. Res., 2008, 38, 71.

[10] D. Öner and T.J. McCarthy, Langmuir, 2000, 16, 7777.

[11] M. Ma and R.M. Hill, Curr. Opin. Colloid Interface Sci., 2006, 11, 193.

[12] A. Nakajima, K. Hashimoto, T. Watanabe, K. Takai, G. Yamauchi, and A. Fujishima, Langmuir, 2000, 16, 7044.

[13] H. Liu, J. Zhai, and L. Jiang, Soft Matter, 2006, $2,811$.

[14] J. H. Bitter, J. Mater. Chem., 2010, 20, 7312.

[15] K. K. S. Lau, J. Bico, K. B. K. Teo, M. Chhowalla, G. A. J. Amaratunga, W. I. Milne, G. H. McKinley, and K. K. Gleason, Nano Lett., 2003, 3, 1701.

[16] B. A. Kakade and V. K. Pillai, J. Phys. Chem. C, 2008, 112, 3183.

[17] S. M. C. Journet, C. Ybert, S. T. Purcell, and L. Bocquet, Europhys. Lett., 2005, $71,104$.

[18] T. Sun, H. Liu, W. Song, X. Wang, L. Jiang, L. Li, and D. Zhu, Angew. Chem., 2004, 116, 4763.

[19] Z. Wang, L. Ci, L. Chen, S. Nayak, P. M. Ajayan, and N. Koratkar, Nano Lett., 2007, 7, 697.

[20] L. Zhang and D.E. Resasco, Langmuir, 2009, 25, 4792.

[21] G. W. T. Sun, H. Liu, L. Feng, and D. Zhu, J. Am. Chem. Soc., 2003, 125, 14996.

[22] K. Gjerde, R. T. R. Kumar, K. N. Andersen, J. Kjelstrup-Hansen, K. B. K. Teo, W. I. Milne, C. Persson, K. Molhave, H. -G. Rubahn, and P. Boggild, Soft Matter 2008, 4, 392. 
[23] H. Gelderblom, Á. G. Marín, H. Nair, A. van Houselt, L. Lefferts, J.H. Snoeijer, and D. Lohse, Phys. Rev. E, 2011, 83, 026306.

[24] P. Tsai, S. Pacheco, C. Pirat, L. Lefferts, and D. Lohse, Langmuir, 2009, 25, 12293.

[25] S. Pacheco Benito and L. Lefferts, J. Colloid Interface Sci., 2011, 364, 530.

[26] D. B. Thakur, R. M. Tiggelaar, J. G. E. Gardeniers, L. Lefferts, and K. Seshan, Surf. Coat. Technol., 2009, 203, 3435.

[27] R. M. Tiggelaar, D. B. Thakur, H. Nair, L. Lefferts, K. Seshan, and J. G. E. Gardeniers, Thin Solid Films, 2013, 534, 341.

[28] N. A. Jarrah, J. G. van Ommen, and L. Lefferts, J. Catal., 2006, 239, 460.

[29] P. D. Rack, Y. Guan, J. D. Fowlkes, A. V. Melechko, and M. L. Simpson, Appl. Phys. Lett., 2008, 92, 223108.

[30] S. J. Randolph, J. D. Fowlkes, A. V. Melechko, K. L. Klein, H. M. Meyer III, M. L. Simpson, and P. D. Rack, Nanotechnol., 2007, 18, 465304.

[31] J. D. Carey, L. L. Ong, and S. R. P. Silva, Nanotechnol., 2003, 14, 1223.

[32] D. B. Thakur, R. M. Tiggelaar, J. G. E. Gardeniers, L. Lefferts, and K. Seshan, Chem. Eng. J., 2012, 227, 128.

[33] R. T. K. Baker, Carbon, 1989, 27, 315.

[34] J. K. Chinthaginjala and L. Lefferts, Carbon, 2009, 47 , 3175.

[35] D. B. Thakur, R. M. Tiggelaar, J. G. E. Gardeniers, L. Lefferts, and K. Seshan, Chem. Eng. J., 2010, 160, 899.

[36] J. Yang, Z. Zhang, X. Men, X. Xu, and X. Zhu, Langmuir, 2010, 26, 10198.

[37] D. B. Thakur, R. M. Tiggelaar, T. M. C. Hoang, J. G. E. Gardeniers, L. Lefferts, and K. Seshan, Appl. Catal. B, 2011, 102, 232.

[38] D. B. Thakur, R. M. Tiggelaar, Y. Weber, J. G. E. Gardeniers, L. Lefferts, and K. Seshan, Appl. Catal. B, 2011, 102, 243. 



\section{Chapter 6}

\section{How water droplets evaporate on a superhydrophobic CNF substrate ${ }^{1, *}$}

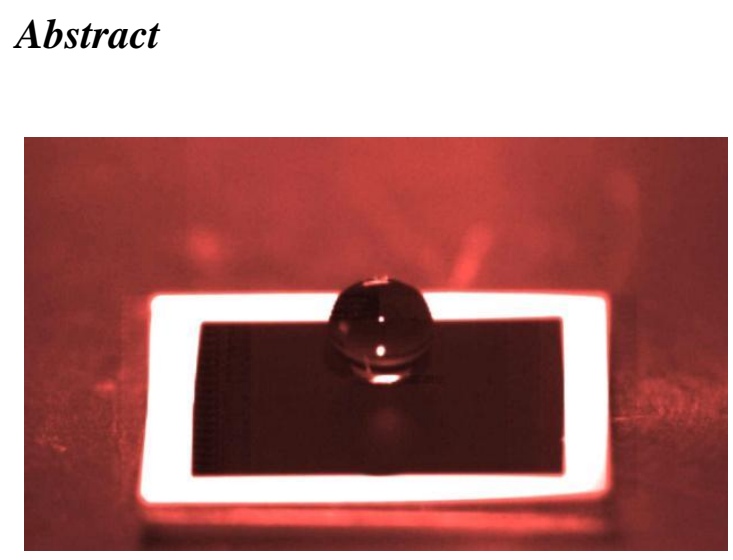

Evaporation of water droplets on a superhydrophobic substrate, on which the contact line is pinned, is investigated. While previous studies focused mainly on droplets with contact angles smaller than $90^{\circ}$, here we analyze almost the full range of possible contact angles $\left(10^{\circ}-150^{\circ}\right)$. The greater contact angles and pinned contact lines can be achieved by use of superhydrophobic carbon nanofiber substrates. The time evolutions of the contact angle and the droplet mass are examined. The experimental data are in good quantitative agreement with the model presented by Popov [Phys. Rev. E, 2005, 71, 036313], demonstrating that the evaporation process is quasistatic, diffusion-driven, and that thermal effects play no role. Furthermore, we show that the experimental data for the evolution of both the contact angle and the droplet mass can be collapsed onto one respective universal curve for all droplet sizes and initial contact angles.

${ }^{1}$ This chapter is published as "How water droplets evaporate on a superhydrophobic substrate" by H.Gelderblom, Á. G. Marin, H. Nair, A. van Houselt, L. Lefferts, J. H. Snoeijer, and D. Lohse, Physical Review E, 2011, 83, 026306.

*Theory of drop evaporation-H. Gelderblom; Drop impact experiments with A.G. Marin. 


\subsection{Introduction}

Evaporation of sessile droplets with small contact angles $\left(<90^{\circ}\right)$ has been studied extensively. Several evaporation modes have been explored: the constant contact-angle mode, ${ }^{[1,2]}$ in which the contact area of the droplet on the substrate vanishes; the constant contact-area mode ${ }^{[1,3-6]}$ in which the contact angle vanishes; and the combination of both modes. ${ }^{[1,7,8]}$ A thorough understanding of droplet evaporation is of vital importance for examining the drying rate ${ }^{[1,3,6,7,9-11]}$ the flow patterns observed inside drying drops, ${ }^{[12-14]}$ and the residual deposits. ${ }^{[5,15,16]}$

In early modeling of evaporating drops, ${ }^{[3,4,7,17]}$ the evaporative flux was assumed to be uniform in the radial direction, as it is for evaporation from a sphere. However, in his study of contact-line deposits, Deegan ${ }^{[5]}$ argued that the evaporative flux from a sessile drop with a spherical cap shape is generally not uniform, but diverges near the edge of the drop for contact angles smaller than $90^{\circ}$. Hu and Larson $^{[6]}$ later used a numerical model to find an expression for the rate of mass loss from a drop in terms of its contact angle, taking this divergence into account. Their model applies to contact angles smaller than $90^{\circ}$.

For larger contact angles, few theoretical descriptions exist for diffusion around a spherical-cap droplet. In Ref. [1], the rate of mass loss was expressed in terms of a series solution, which can be approximated in both the small and large contact-angle regimes. Popov ${ }^{[16]}$ described an analytical solution for the rate of mass loss in terms of the contact angle, which applies to the full range of contact angles. However, this model has never been validated against experimental data in the large contact-angle regime.

Apart from the diffusive spreading of water vapor described by the models mentioned earlier, there are other factors that may influence the evaporation rate (see, e.g., Ref.[18] for an overview). First, the evaporation models discussed assume a stationary contact line. When the contact line is moving, dynamic effects may complicate the problem for both the vapor concentration outside and the viscous flow inside the drop ${ }^{[8]}$. Secondly, evaporative cooling of the drop can reduce the evaporation rate. ${ }^{[9-11]}$ The resulting temperature gradients on the drop surface can induce a Marangoni flow, ${ }^{[13,14]}$ and can give rise to a Marangoni-Bénard instability. ${ }^{[19]}$ 
Finally, in addition to the diffusion of water vapor, free convective transport may play a role, increasing the evaporation rate. ${ }^{[11,20]}$ However, the influence of these factors on the evaporation rate has yet to be confirmed experimentally.

In this chapter, we describe our investigation into the evaporation of water droplets on carbon nanofiber (CNF) substrates; see Figure 1.

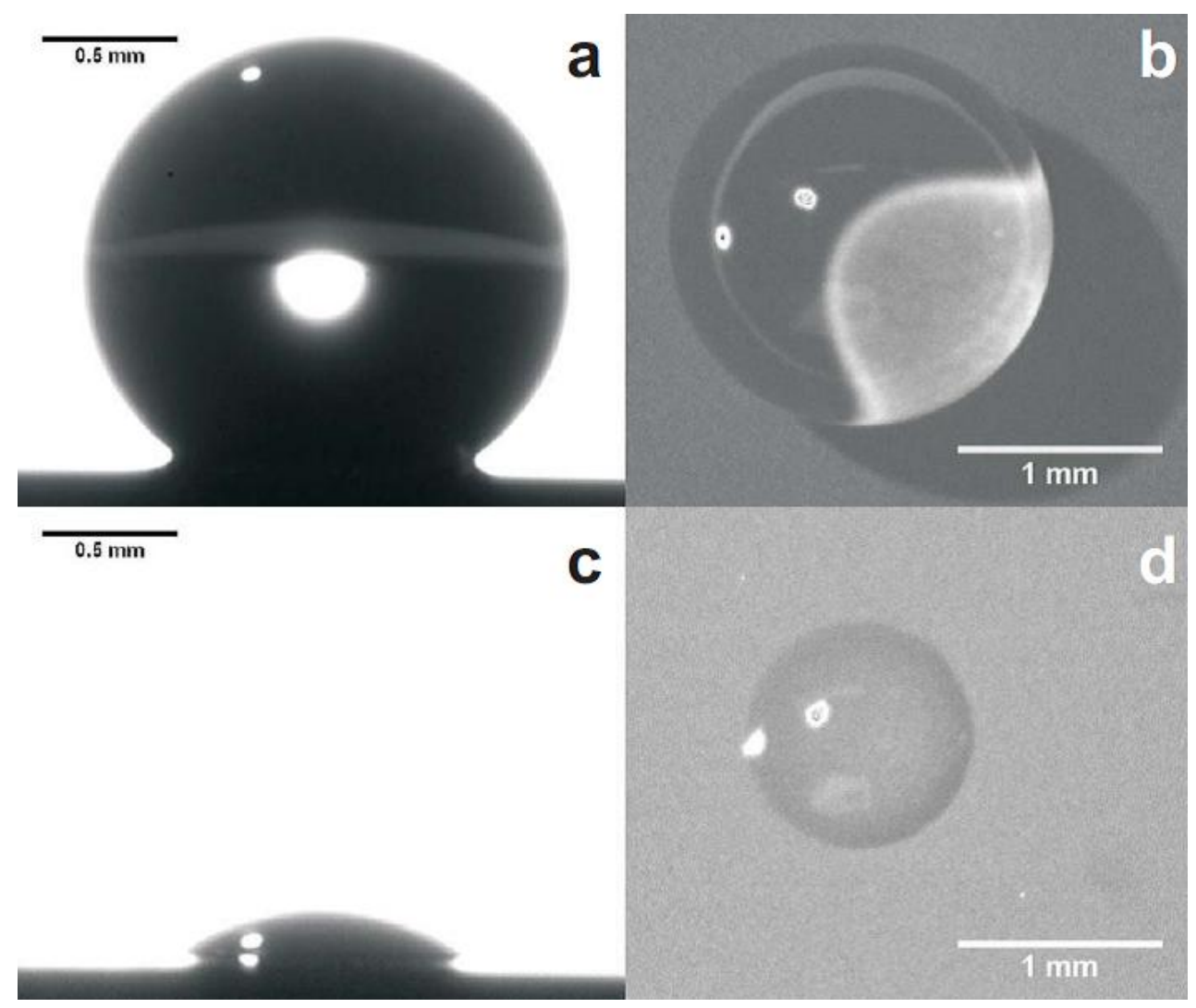

Figure 1: Side-view (a) and top-view (b) of an $8 \mu \mathrm{L}$ droplet on a CNF substrate in the initial moments. Parts (c) and (d) show the same droplet in the last moments before being completely evaporated. Note that the contact line remains perfectly circular and completely pinned until almost the end of the process.

These substrates belong to the family of ordered carbonaceous structures: the graphitic planes are oriented under an angle to the central axis. CNF substrates can exhibit superhydrophobicity. ${ }^{[21]}$ The samples used here have contact angles with water, ranging from $150^{\circ}$ up to $170^{\circ}$. On superhydrophobic substrates, all evaporation modes can occur. The constant contact-angle mode is mostly observed when the contact-angle hysteresis is low; the constant contact-area mode is mostly observed 
when the hysteresis is high. ${ }^{[22,23]}$ On our CNF substrates, the contact line remains pinned throughout almost the entire experiment; hence evaporation takes place in the constant contact-area mode. In contrast, superhydrophobic substrates based on micropillar arrays display contact-line jumps during evaporation. ${ }^{[24,25]}$ Because we consider pinned contact lines, we can study evaporating drops in almost the full range of possible contact angles $\left(0^{\circ}-150^{\circ}\right)$. The rate of mass loss and contact-angle evolution over time are obtained experimentally for various drop sizes. We show that the evaporation dynamics is described accurately by the diffusion-based model of Popov, ${ }^{[16]}$ suggesting that thermal and free-convection effects are unimportant in our experiment. In addition, we show that the evolutions of the droplet mass and contact angle can be described by a universal relation, that is, independent of the drop size and initial contact angle.

In section 6.2, the experimental setup and preparation of the CNF substrates are described. The experimental results are discussed in section 6.3.1. The theoretical model for droplet evaporation adopted from Popov ${ }^{[16]}$ is briefly described in section 6.3.2. Finally, in section 6.3.3, it is shown that the theoretical results are in good quantitative agreement with the experimental data.

\subsection{Experimental Methods}

\subsubsection{Preparation of the CNF substrates}

The droplets were left evaporating in an empty room ${ }^{2}$ at a constant temperature of $23{ }^{\circ} \mathrm{C}$ and a humidity of $30 \%$ over CNF substrates; see Figure 2. CNFs were grown on oxidized silicon substrates using a Ni thin film as a catalyst. A 250-nm-thick $\mathrm{SiO}_{2}$ layer was grown on p-type $\mathrm{Si}(001)$ via wet oxidation. On top of this oxide layer, $10 \mathrm{~nm}$ Ta was deposited followed by a 25-nm-thick Ni layer. The samples were pretreated prior to the CNF synthesis in a quartz reactor. The substrates were placed on a flat quartz boat positioned centrally inside a quartz reactor, and the

\footnotetext{
${ }^{2}$ No human heat sources were present.
} 
temperature was increased at a rate of $5 \mathrm{~K} \cdot \mathrm{min}^{-1}$ from room temperature up to $500{ }^{\circ} \mathrm{C}$ in a $\mathrm{N}_{2}(99.999 \%$, Indugas) atmosphere. During this pretreatment step, the samples were subjected to 20 vol.\% of $\mathrm{H}_{2}$ in $\mathrm{N}_{2}$ at a total flow rate of $50 \mathrm{~mL} \cdot \mathrm{min}^{-1}$ at $500{ }^{\circ} \mathrm{C}$ for $2 \mathrm{~h}$; then the temperature was increased up to $635{ }^{\circ} \mathrm{C}$. At $635{ }^{\circ} \mathrm{C}, 25$ vol.\% ethylene (99.95\% Praxair) in $\mathrm{N}_{2}$ was passed through the reactor for $1 \mathrm{~h}$, while 6.25 vol.\% $\mathrm{H}_{2}$ (99.999\%, Indugas) was added for the first minute of the reaction time. After the reaction time, the substrates were cooled down in $\mathrm{N}_{2}$ at a rate of $10{ }^{\circ} \mathrm{C} \cdot \mathrm{min}^{-1}$ until room temperature was reached. The CNF samples were used without further functionalization.

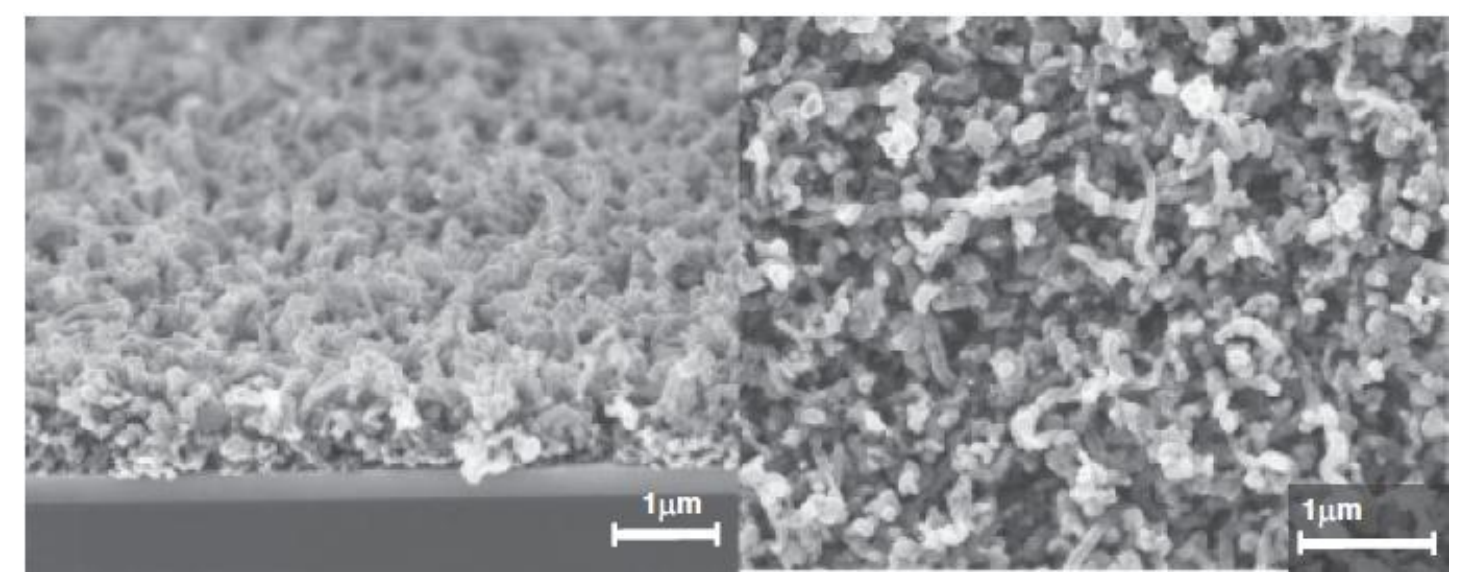

Figure 2: Scanning electron microscopy (SEM) images of the CNFs used as superhydrophobic substrates. Tilted side-view (left) and augmented top-view (right).

\subsubsection{Measurement of droplet evaporation}

To analyze the evaporation of droplets on CNF substrates, the droplets were observed during their total evaporation time and photographed at 1-s time intervals. Two synchronized cameras (Lumenera Lm135, $1392 \times 1040$ pixels) were used for this purpose, one taking side-view images and other taking top-view images; see Figure 1. Side-view images allowed us to compute volume (mass), contact angle, area, droplet radius, mass loss, and spreading velocity at every instant. The image analysis was performed using a custom-made MATLAB code in which the detected droplet profile was fitted to an ellipse. The droplets considered in this study are much smaller than the capillary length (which is $2.7 \mathrm{~mm}$ for a water droplet ${ }^{[18]}$ ), hence we can neglect flattening of the drops by gravity. Nevertheless, we used an elliptical rather than a 
spherical fitting. The elliptical fit allowed us to use three fitting parameters (two semiaxes and the angle of the ellipse with the horizontal plane) instead of only one (droplet radius), thereby increasing the precision of the determination of the volume and contact angle of the droplets. The ellipticity of the droplets, defined as the ratio between both semi-axes, was always below $7 \%$.

The contact line of the droplets was detected automatically; the contact angles were then measured by finding the tangent of the ellipse at the contact line. The error in the determination of the contact angle, based on the quality of the fits, was found to be less than $1 \%$. The volume of the droplet was obtained by calculating the ellipse area above the contact line and assuming rotational symmetry with respect to the vertical axis, with an error below 10\%. The rate of mass loss was computed applying a fourth-order finite differentiation of the ellipse volume over time.

Top-view images were used to obtain qualitative information on the stability and circular symmetry of the contact line; using this information, we rejected those few experiments in which the contact line had a highly irregular shape.

Due to the chaotic three-dimensional distribution of the nanofibers, the way the liquid wets the structure is more complex than for ordered superhydrophobic microstructures, ${ }^{[24,25]}$ for which two wetting states can be defined: the Cassie-Baxter state, in which the contact of the liquid with the substrate is minimum, and the Wenzel state, in which the contact is maximum. In our case, it is assumed that the liquid remains in a mixed state and that the transitions from one intermediate state to another are sufficiently smooth to be undetectable. Therefore, we will not use this terminology in this paper.

\subsection{Results and Discussions}

\subsubsection{Experimental results}

The droplet volume, contact angle, and radius were determined from the experimental data with a time resolution of $1 \mathrm{~s}$. The droplet volume versus time plot clearly shows nonlinear behavior; see Figure 3a. Hence, a model based on the small contact-angle approximation, which predicts the droplet volume to decrease linearly in time, ${ }^{[5,6]}$ will not suffice to describe the evolution of the droplet volume over time. 
From the droplet volume measurements, the rate of mass loss of the droplet $d M / d t$ was derived, as described in Section 6.2.2. Figure 4a shows that $d M / d t$ decreases with decreasing contact angle, hence it also decreases in time. Again, nonlinear behavior is observed, with a steep decline for larger contact angles, but this levels off to a constant rate of mass loss for contact angles smaller than $70^{\circ}$.
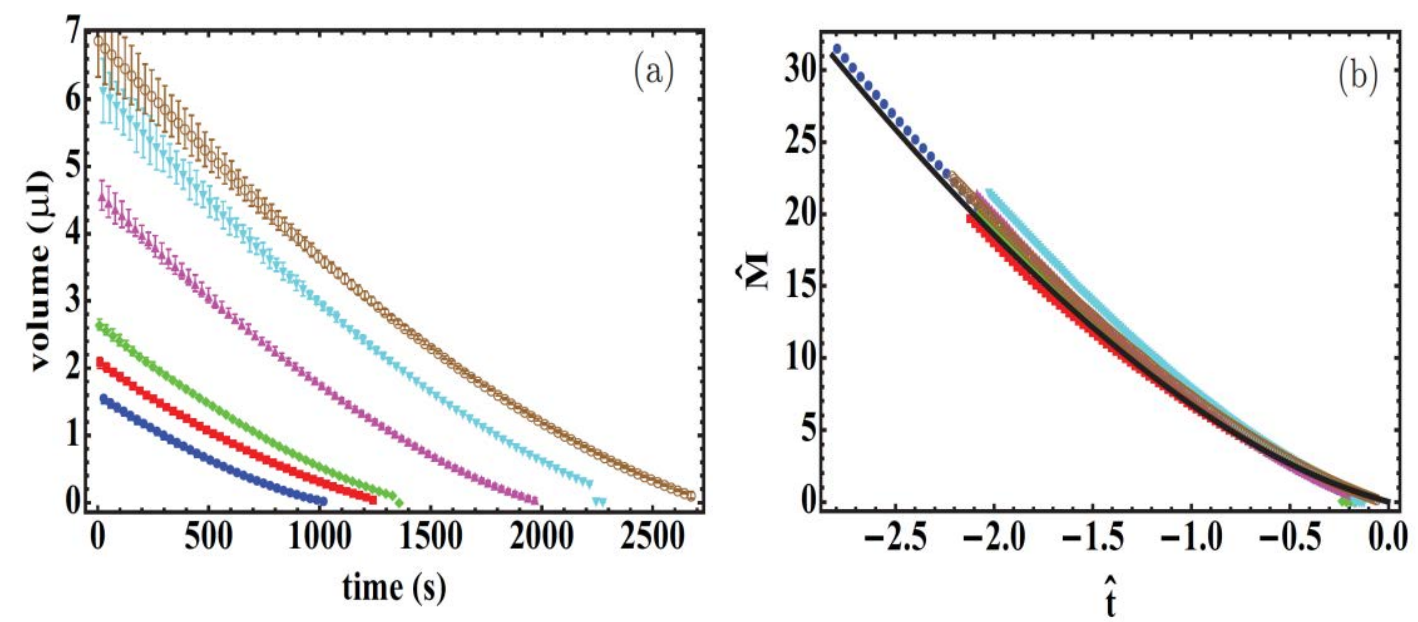

Figure 3: (a) Droplet volume versus time for initial droplet volumes of $1.6 \mu \mathrm{L}$ (blue filled circles), $2.1 \mu \mathrm{L}$ (red squares), $2.9 \mu \mathrm{L}$ (green diamonds), 4.6 $\mu \mathrm{L}$ (magenta upward triangles), $6.2 \mu \mathrm{L}$ (cyan downward triangles), and $6.9 \mu \mathrm{L}$ (brown unfilled circles). The error bars are deduced from the elliptical fit to the data. The measurements were performed with a time resolution of $1 \mathrm{~s}$, but for clarity we show the data with a 30-s resolution. (b) The dimensionless droplet mass plotted against the dimensionless time. The black solid line represents the theoretical prediction according to the Popov model. The experimental data is scaled according to equation (6). The time is set to 0 at the end of the droplet life (see text).

During the evaporation, the contact angle of the droplets decreases over time from about $150^{\circ}$ to $0^{\circ}$, as shown in Figure 5a. Initially, the contact angle decreases slowly over time. This is followed by a more rapid, linear decrease over time when the contact angle becomes smaller than approximately $70^{\circ}$. The initial contact angles of the droplets differ somewhat owing to the irregularities in the substrate. For comparison, not only the experimental data but also the predictions based on the Popov model are shown in Figure 5a. A more detailed explanation of this model is given in Section 6.3.2. 

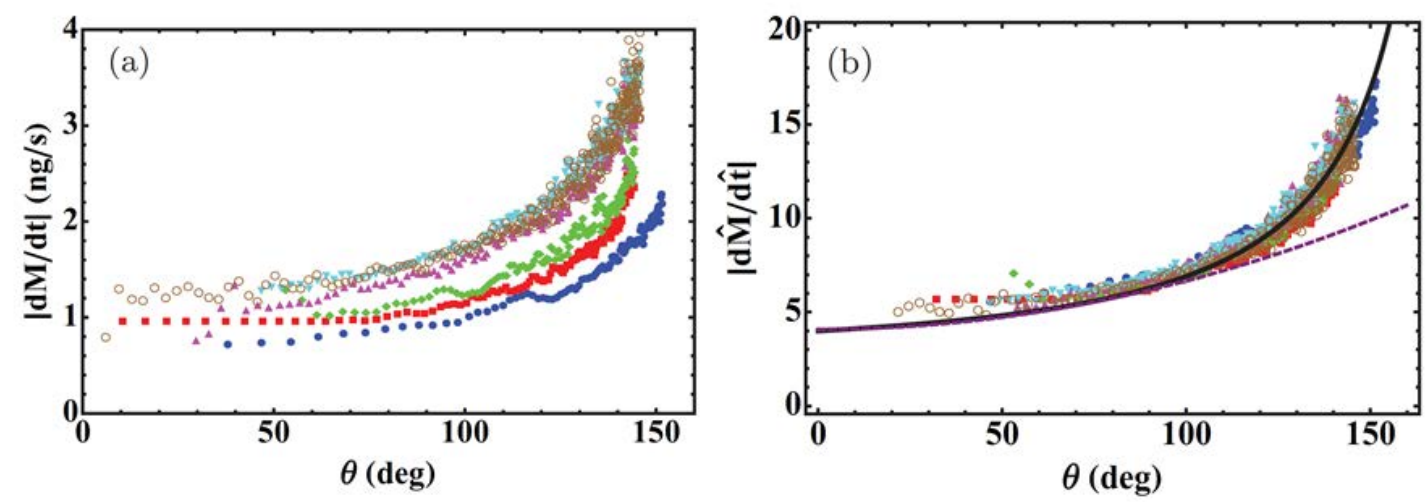

Figure 4: (a) The rate of mass loss of the droplet (derived from the measured droplet volume) versus the contact angle. Colors and markers are as in Figure 3. (b) The same data, but now scaled according to equation (6). Predictions from the Popov model (black solid line) and the model of Hu and Larson (purple dashed line) are shown.
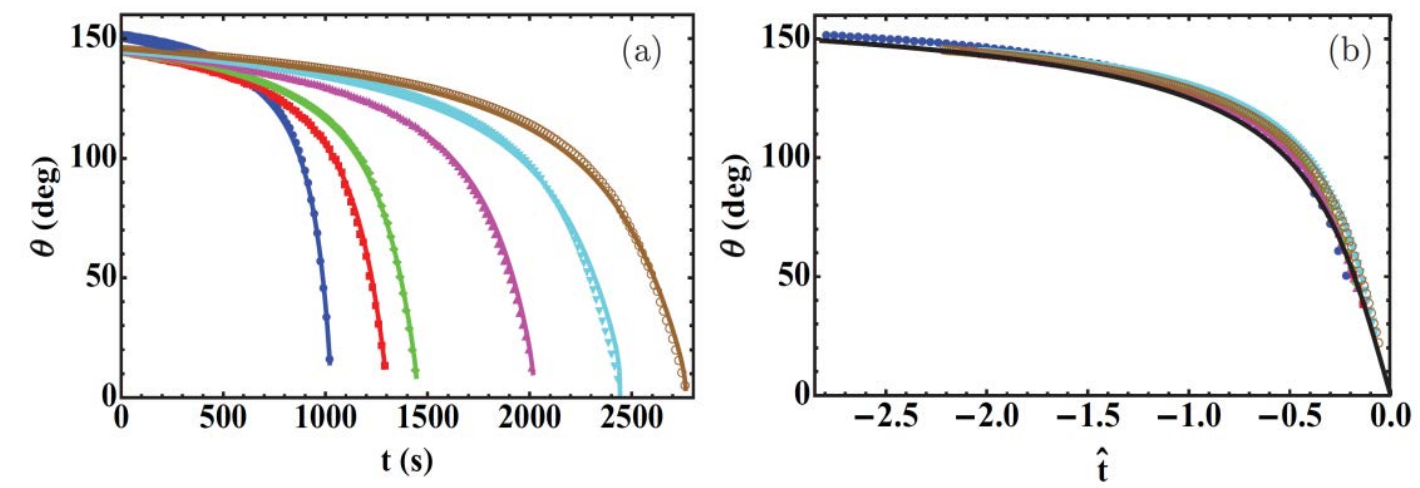

Figure 5: (a) The evolution of the contact angle over time. The experimental data (•) can be described very well by the theoretical model of Popov ( - ) by adjusting the drop radius according to its experimental value (see section 6.3.3.). The error in the experimental data is not shown, since it is below $1 \%$. (b) The same data, but with the time scaled according to equation (6) and set to 0 at the end of the droplet life (see text). The black solid line represents the theoretical prediction according to the Popov model. Colors and markers are as in Figure 3.

One advantage of the CNF substrates is that the contact lines of the droplets remain pinned throughout almost the entire experiment. Therefore, droplet evaporation in the constant contact-area mode can be studied, in the absence of any contact-line dynamics. Similar behavior of the contact angle in the pinned situation has been reported for natural lotus leaves, ${ }^{[23]}$ synthetic superhydrophobic surfaces with high contact-angle hysteresis, ${ }^{[22]}$ and aligned carbon nanotube (CNT) samples. ${ }^{[26]}$ 
Figure 6 shows that depinning only occurs during the final moments of the droplet's life. In the depinning phase, the contact angle is typically smaller than $40^{\circ}$. Once the droplet starts to depin, the measurement error shoots up. This is because the contact line does not depin homogeneously, and it is therefore no longer exactly circular.

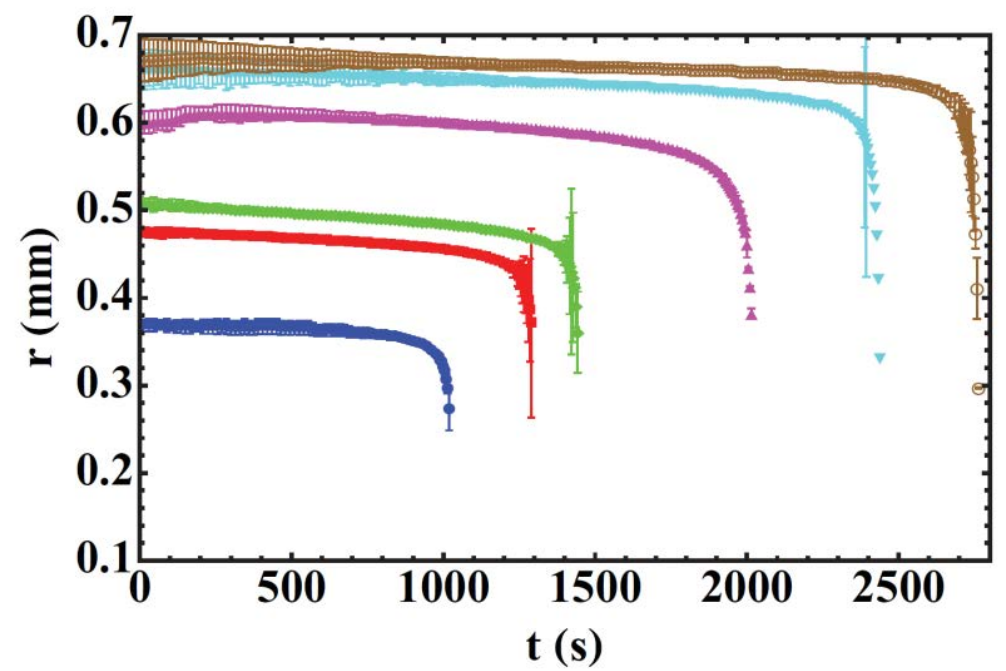

Figure 6: The droplet radius vs. time. Significant depinning of the contact line is observed during the final $4 \%$ of the droplet lifetime. Data are shown with 15-s time resolution. During the depinning, a resolution of $5 \mathrm{~s}$ is used. Colors and markers are as in Figure 3.

\subsubsection{Theory of droplet evaporation}

To describe theoretically the measured time evolution of a droplet's contact angle and mass, we need to know the evaporative flux from the droplet surface. This flux depends on the rate-limiting step in the vapor transport. We assume that vapor transport by free convection, induced by the density difference between dry and humid air, ${ }^{[20]}$ is negligible compared to diffusive transport. The influence of evaporative cooling of the droplet on the evaporation rate ${ }^{[11]}$ is also neglected. Hence, the vapor transport occurs mainly by diffusive spreading of the water vapor in air, and is characterized by diffusion time

$$
t_{d}=R^{2} / D
$$

with $R$ the droplet radius in the plane of the substrate and $D$ the diffusion coefficient. The diffusion time for water vapor in air is of the order of $10^{-2} \mathrm{~s}$. The evaporation occurs in a quasisteady fashion: the time scale for diffusion is much smaller than the 
typical droplet evaporation time $\left(t_{e}\right)$. As will become clear from the dimensional analysis presented in equation (6),

$$
t_{e}=\rho /\left(c_{s}-c_{\infty}\right) d
$$

In essence, $t_{e}$ can be estimated by comparing the initial droplet mass, proportional to the droplet density $(\rho)$, to the rate of mass loss, proportional to $c_{s}-c_{\infty}$, the vapor concentration difference between the drop surface and the surroundings. Here,

$$
t_{e} / t_{d}=\rho / c_{s}-c_{\infty}
$$

is of the order of $10^{5}$. We do not take into account the Kelvin correction to the vapor pressure because this effect is negligible for droplets of the size considered here.

To determine the diffusive outflux from the drop surface, the vapor concentration field around the droplet has to be calculated. We follow the approach taken by Popov. ${ }^{[16]}$ For completeness, we briefly formulate the problem in the following paragraph.

A cylindrical coordinate system $(r, z, \phi)$ is adopted, with $r$ being the radial coordinate, $z$ the direction normal to the substrate, and $\phi$ the circumferential coordinate. The origin of this system is chosen such that $z=0$ corresponds to the substrate and $r=0$ to the center of the droplet. In this case, the problem is axisymmetric, that is, $\phi$-independent. In the quasisteady, diffusion-limited case, the concentration field $c(r, z)$ around the droplet is given by

$$
\nabla^{2} c=0
$$

The boundary conditions imposed along the spherical-cap-shaped droplet with arbitrary contact angle $\theta$ are

(i) $\quad c=c_{S}$, the saturated vapor concentration, along the droplet surface;

(ii) $c=c_{\infty}$, the ambient vapor concentration, far away from the drop; and

(iii) the substrate is impermeable, hence $\partial c / \partial z=0$ along the substrate.

The diffusive flux is given by

$$
J=-D \nabla c .
$$


In our experiments, the ambient temperature was $23{ }^{\circ} \mathrm{C}$ and the humidity $H=0.3$. At this temperature, $D=24.6 \times 10^{-6} \mathrm{~m}^{2} \cdot \mathrm{s}^{-1}, \rho=997.6 \mathrm{~kg} \cdot \mathrm{m}^{-3}$, and $c_{S}=2.08 \times 10^{-2} \mathrm{~kg} \cdot \mathrm{m}^{-3}$ (obtained from (Ref. [27], pp.6-1, 6-191) by linear interpolation); furthermore,

$$
c_{\infty}=H c_{s} .
$$

In the limit of small contact angles, simplified solutions to Equation (1) subject to the boundary condition (i)-(iii) can be used, as presented by Deegan ${ }^{[5]}$ and $\mathrm{Hu}$ and Larson. ${ }^{[6]}$ In our case, a more advanced model is needed because we consider droplets evaporating on a superhydrophobic substrate, with initial contact angles of approximately $150^{\circ}$. The analytical solution to the equivalent problem of finding the electric potential around a charged lens-shaped conductor is described in Ref. [28]. Popov $^{[16]}$ used this result to determine the rate of mass loss from a droplet of arbitrary contact angle:

$$
\begin{aligned}
\frac{d M}{d t} & =-\int_{0}^{R} J(r) \sqrt{1+\left(\partial_{r} h\right)^{2}} 2 \pi r d r \\
& =-\pi R D\left(c_{s}-c_{\infty}\right) f(\theta)
\end{aligned}
$$

with $M$ the droplet mass, $J$ the diffusive outflux from the droplet surface, $h(r, t)$ the droplet height, $t$ the time, and

$$
f(\theta)=\frac{\sin \theta}{1+\cos \theta}+4 \int_{0}^{\infty} \frac{1+\cosh 2 \theta \tau}{\sin 2 \pi \tau} \tanh [(\pi-\theta) \tau] d \tau .
$$

The droplet mass can be expressed in terms of $\theta$ by the geometric relation

$$
M(\theta)=\rho \pi R^{3} \frac{\cos ^{3} \theta-3 \cos \theta+2}{3 \sin ^{3} \theta},
$$

which yields an ordinary differential equation for $\theta$ as a function of $t$,

$$
\frac{d \theta}{d t}=-\frac{D\left(c_{s}-c_{\infty}\right)}{\rho \mathrm{R}^{2}}(1+\cos \theta)^{2} f(\theta)
$$

Numerical integration then gives $\theta$ as a function of $t$. Once $\theta$ is known, $M(\theta)$ and $\partial M / \partial t$ can be derived.

In Figures 3a-5a, we showed the evolution of the droplet mass and contact angle in time for various drop sizes. Based on the theory just described, one would expect a universal behavior that is independent of the drop size and the other problem 
parameters $c_{S}, H, \rho$, and $D$. To demonstrate this, we introduce the non-dimensional mass and time as

$$
\widehat{M}=\frac{M}{\rho R^{3}}, \quad \hat{t}=\frac{c_{S}-c_{\infty}}{\rho} \frac{t}{R^{2} / D} .
$$

By substituting (6) into Equations (2)-(5), we obtain

$$
\begin{gathered}
\frac{d \widehat{M}}{d t}=-\pi f(\theta), \\
\widehat{M}=\pi \frac{\cos ^{3} \theta-3 \cos \theta+2}{3 \sin ^{3} \theta}, \\
\frac{d \theta}{d \hat{t}}=-(1+\cos \theta)^{2} f(\theta) .
\end{gathered}
$$

The relations (7)-(9) no longer depend on the size of the droplets, but only on the contact angle. This implies that when we rescale the experimental data according to (6), they should all collapse onto the theoretical curves described by (7)-(9).

\subsubsection{Comparison between theory and experiment}

In Section 6.3.2, we explained that it should be possible to collapse the experimental data for all droplet sizes measured onto a single theoretical curve. To test this, we have to scale the experimental data according to (6). As a characteristic length scale, we would like to use the droplet radius. However, during the final moments of the droplet's lifetime, the droplet radius is a time-dependent quantity. Therefore, we discarded all data in which the droplet radius was changing significantly $(>10 \%)$ in the results that follow, and we used the initial droplet radius for scaling.

The most direct prediction from the Popov model, which involves no time integration, is the dependence of the rate of mass loss on the contact angle (7). Indeed, the scaled experimental data collapse onto a single curve, which is in excellent agreement with the theoretical prediction (7) as shown in Figure 4b. For comparison, the result obtained from applying the model of $\mathrm{Hu}$ and Larson ${ }^{[6]}$ is also shown. Their approximation works well up to $\theta=90^{\circ}$, but for larger contact angles Popov's fully analytical model is required to adequately describe the data. 
Figure 5b shows that the experimental data for the contact angle versus (dimensionless) time follow a universal theoretical curve for all droplet sizes measured. The total time it takes a droplet to evaporate depends on its initial contact angle, as explained in Section 6.3.2. Since the initial contact angles vary somewhat, the droplet lifetimes differ. However, the experimental time is not an absolute measure, and we therefore have the freedom to set $t=0$ at whichever contact angle we want. As the reference point, we chose $t=0$ at the end of the evaporation process, which is characterized by $\theta=0$. This point is found by linear extrapolation from the last data points measured to $\theta=0$.

Once the contact angle in time is known, we can apply relation (8) to derive the droplet mass theoretically. Experimentally, the droplet mass is obtained independently of the contact angle. Therefore, the comparison between the theoretical predictions and the experimental data for the droplet mass, as in Figure 3b, provides a second validation of the model.

In the results just described, we used the experimental data as long as the contact angle remained pinned and hence the droplet radius remained constant. In Figure 6, we showed that depinning occurs during the final moments of the droplet's lifetime. To construct the theoretical curves in Figure 5a, this radius change has been taken into account. Time integration was performed backward in time, starting from the smallest contact angle measured. The agreement between the model results and the experimental data is surprisingly good, even in the regime where the droplet radius is changing significantly. Although the droplet radius decreases rapidly, the time scale over which the radius shrinks is still large - of the order of $100 \mathrm{~s}-$ compared to diffusion time $\left(10^{-2} \mathrm{~s}\right)$. Therefore, contact-line dynamics is still of negligible influence, and the quasisteady evaporation model can indeed be applied. $^{[8,18]}$

\subsection{Conclusion}

Evaporation of water droplets on superhydrophobic CNF substrates is studied. These substrates allowed us to measure the evolution of droplet mass and contact angle over time, while the contact line remained pinned throughout almost the entire 
experiment. The initial contact angle was as high as $150^{\circ}$, and since it decreases to $0^{\circ}$ during evaporation, a very large range of contact angles could be studied. Therefore, CNF substrates are a very useful tool to study droplet evaporation in the absence of contact-line dynamics. In our theoretical analysis, we deduced universal relations for the time evolutions of the droplet mass and contact angle. This universal scaling behavior is confirmed by our experimental results. Since the experimental data covered almost the entire range of possible contact angles, we have been able to validate the diffusion-based analytical evaporation model presented by Popov. ${ }^{[16]}$ The agreement of our experimental data with this theoretical model - that does not contain any adjustable parameters-is excellent. Therefore, we conclude that in our experiments the evaporation is quasistatic and diffusion-driven, and thermal effects play no role.

Even during the brief depinning phase, the quasisteady model predicted the experimental data surprisingly well. Hence, a pinned contact line is not a stringent requirement for the applicability of the quasisteady evaporation model, provided that the radius change takes place on a longer time scale than the diffusion. In contrast, for droplets evaporating on complete wetting substrates, a quasistatic droplet profile can no longer be assumed and viscous effects influence the evolution of the contact angle over time. ${ }^{[8,29,30]}$ It would be interesting to address intermediate cases, in which there is some contact-line motion, so as to establish the range of applicability of the quasisteady evaporation model.

\subsection{References}

[01] R. G. Picknett and R. Bexon, J. Colloid Interface Sci.,1977, 61, 336.

[02] H. Y. Erbil, G. McHale, and M. I. Newton, Langmuir, 2002, 18, 2636.

[03] K. S. Birdi, D. T. Vu, and A. Winter, J. Phys. Chem., 1989, 93, 3702.

[04] S. M. Rowan, M. I. Newton, and G. McHale, J. Phys. Chem., 1995, 99, 13268.

[05] R. D. Deegan, O. Bakajin, T. F. Dupont, G. Huber, S. R. Nagel, and T. A. Witten, Phys. Rev. E, 2000, 62, 756.

[06] H. Hu and R. G. Larson, J. Phys. Chem. B, 2002, 106, 1334. 
[07] C. Bourges-Monnier and M. Shanahan, Langmuir, 1995, 11, 2820.

[08] M. Cachile, O. Benichou, C. Poulard, and A. M. Cazabat, Langmuir, 2002, 18, 8070.

[09] S. David, K. Sefiane, and L. Tadrist, Colloids Surf. A, 2007, 298, 108.

[10] G. J. Dunn, S. K. Wilson, B. R. Duffy, S. David, and K. Sefiane, Colloid Surf. A, 2008, 323, 50.

[11] G. J. Dunn, S. K. Wilson, B. R. Duffy, S. David, and K. Sefiane, J. Fluid Mech., $2009, \mathbf{6 2 3}, 329$

[12] H. Hu and R. G. Larson, Langmuir, 2005, 21, 3963.

[13] H. Hu and R. G. Larson, Langmuir, 2005, 21, 3972.

[14] W. D. Ristenpart, P. G. Kim, C. Domingues, J. Wan, and H. A. Stone, Phys. Rev. Lett., 2007, 99, 234502.

[15] R. D. Deegan, O. Bakajin, T. F. Dupont, G. Huber, S. R. Nagel, and T. A. Witten, Nature (London), 1997, 389, 827.

[16] Y. O. Popov, Phys. Rev. E, 2005, 71, 036313.

[17] H. Y. Erbil and R. A. Meric, J. Phys. Chem. B, 1997, 101, 6867.

[18] A. M. Cazabat and G. Guena, Soft Matter, 2010, 6, 2591.

[19] V. X. Nguyen and K. J. Stebe, Phys. Rev. Lett., 2002, 88, 164501.

[20] N. Shahidzadeh-Bonn, S. Rafai, A. Azouni, and D. Bonn, J. Fluid Mech., 2006, 549, 307.

[21] P. Tsai, S. Pacheco, C. Pirat, L. Lefferts, and D. Lohse, Langmuir, 2009, 25, 12293.

[22] S. A. Kulinich and M. Farzaneh, Appl. Surf. Sci., 2009, 255, 4056.

[23] X. Zhang, S. Tan, N. Zhao, X. Guo, X. Zhang, Y. Zhang, and J. Xu, Chem. Phys. Chem., 2006, 7, 2067.

[24] M. Sbragaglia, A. M. Peters, C. Pirat, B. M. Borkent, R. G. H. Lammertink, M. Wessling, and D. Lohse, Phys. Rev. Lett., 2007, 99, 156001.

[25] P. Tsai, R. G. H. Lammertink, M. Wessling, and D. Lohse, Phys. Rev. Lett., 2010, 104, 116102.

[26] K. Gjerde, R. T. Rajendra-Kumar, K. Nordstrom-Andersen, J. Kjelstrup-Hansen, K. B. K. Teo, W. I. Milne, C. Persson, K. Molhave, H.-G. Ruabahn, and P. Boggild, Soft Matter, 2008, 4, 392. 


\section{Chapter 6}

[27] D. R. Lide, CRC Handbook of Chemistry and Physics, 2009, 90th ed. (CRC, Boca Raton, FL).

[28] N. N. Lebedev, Special Functions and their Applications (1965, Prentice-Hall, Englewood Cliffs, NJ).

[29] C. Poulard, G. Guena, A. M. Cazabat, A. Boudaoud, and M. B. Amar, Langmuir, $2005,21,8226$.

[30] J. Eggers and L. M. Pismen, Phys. Fluids, 2010, 22, 112101.

$* * * * * * * * * * * *$ 


\section{Chapter 7}

\section{The Leidenfrost temperature increase for impacting droplets on carbon-nanofiber surfaces ${ }^{1}$}

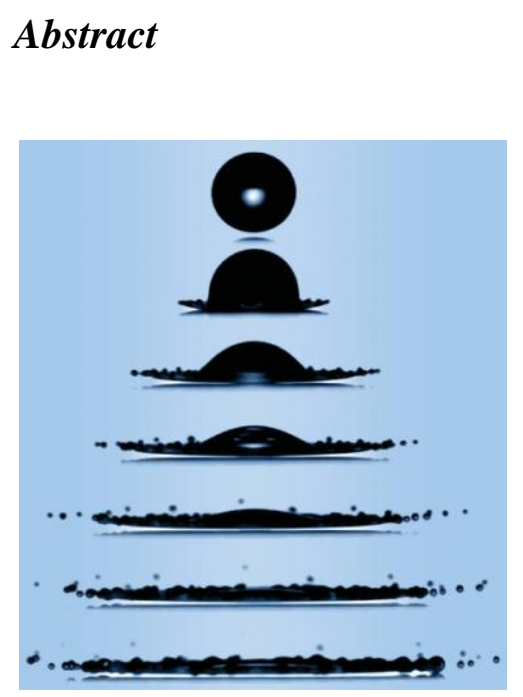

Droplets impacting on a superheated surface can either exhibit a contact boiling regime, in which they make direct contact with the surface and boil violently, or a film boiling regime, in which they remain separated from the surface by their own vapor. The transition from the contact boiling to the film boiling regime depends not only on the temperature of the surface and the kinetic energy of the droplet, but also on the size of the structures fabricated on the surface. Here we experimentally show that surfaces covered with carbonnanofibers delay the transition to film boiling to much higher temperatures compared to smooth surfaces. We present physical arguments showing that, because of the small scale of the carbon fibers, they are cooled by the vapor flow just before the liquid impact, thus permitting contact boiling up to much higher temperatures than on smooth surfaces. We also show that as long as the impact is in the film boiling regime, the spreading factor of impacting droplets is consistent with the We $e^{3 / 10}$ scaling (with We being the Weber number) as predicted for large We by a scaling analysis.

\footnotetext{
${ }^{1}$ This chapter is published as "The Leidenfrost temperature increase for impacting droplets on carbonnanofiber surfaces" by H. Nair and H. J. J. Staat, T. Tran, A. van Houselt, A. Prosperetti, D. Lohse and C. Sun, Soft Matter, 2014, 10, 2102.
} 


\subsection{Introduction}

Spray cooling is an effective heat transfer mechanism as it is capable of delivering spatially uniform and high heat transfer rates. ${ }^{[1-3]}$ An important new application of this technology is in electronic cooling, where the growing power consumption and decreasing sizes pose increasingly challenging heat dissipation demands. ${ }^{[4,5]}$ Other common situations in which cold drops impact hot surfaces are found in internal combustion engines, ${ }^{[6,7]}$ quenching of aluminum and steel, ${ }^{[8]}$ fire suppression $^{[9,10]}$ and others.

In all these applications a stream of fine droplets dispensed, e.g., from a nozzle impinges on a solid surface and cools it by a combination of sensible heat absorption and latent heat of vaporization. Due to the inherent complexity of the phenomenon and the large number of parameters involved, such as droplet size, velocity distribution, droplet number density and material properties, many aspects of the physical mechanisms involved still remain incompletely understood. ${ }^{[1,11,12]}$

A fundamental understanding of the impact of an individual droplet on superheated surfaces is the first step toward a better understanding and eventual optimization of the process. Various aspects of this particular problem have been investigated, such as the effect of droplet size, velocity, physical properties, ${ }^{[13,14]}$ and surface roughness, ${ }^{[15,16]}$ the transition between different boiling regimes, ${ }^{[16-21,31]}$ the surface temperature change and heat transfer during impact, ${ }^{[15,22-24]}$ the residence time of the impacting droplet, ${ }^{[16,25]}$ the spreading factor ${ }^{[16,21,26]}$ and others.

An important quantitative feature of the phenomenon is the transition temperature $T_{L}$ between the contact boiling regime, where the liquid makes direct contact with the heated surface, and the film boiling regime, where a stable vapor layer between the liquid and the surface is formed during impact. As the rate of heat transfer in the film boiling regime is significantly reduced due to the poor thermal conductivity of the vapor layer, this regime should be avoided for applications that require high heat transfer rates. Methods to increase $T_{L}$, or delay the onset of the film boiling regime, are therefore of great interest for such applications. 
Recently, surfaces covered with nanofibers were shown to effectively enhance the heat transfer from the surface to a liquid in contact with it. ${ }^{[24,27]}$ In particular, it was reported that for impacting ethanol droplets on surfaces covered with nanofiber mats, the film boiling behavior was not observed even when the surface temperature was as high as $300{ }^{\circ} \mathrm{C},{ }^{[23]}$ which implies that the transition temperature to film boiling is increased compared to that observed on smooth surfaces. This is in marked contrast to the impact on surfaces covered with microstructures, for which the transition temperature is considerably decreased compared to a smooth surface. ${ }^{[16]}$ Indeed, numerous questions regarding the effects of nanostructures on the transition temperature are still open. First of all, why do nanofibers cause a higher $T_{L}$ compared to that on smooth surfaces? And, further, what is the transition temperature $T_{L}$ for this type of surfaces? How does it change with the size of the nanostructures on the surfaces?

To answer these questions, in this paper we study the impact of droplets on heated surfaces covered with carbon nanofibers (CNFs), which are carbonaceous structures grown by catalytic vapor deposition of hydrocarbons. This type of nanostructure is well-known for its unique physical and chemical properties with a tunable morphology (the diameter can be varied from a few to hundreds of nanometers; the height can be controlled from a few micrometers to millimeters), which in turn can be exploited for tuning the roughness, porosity, and surface area. ${ }^{[28]}$

We use two types of CNF surfaces corresponding to two different typical fiber lengths and a smooth silicon surface. For each type of surface, we determine the transition temperature and its dependence on the impact velocity. We propose a quantitative explanation of the effect of nanofibers on the transition temperature $T_{L}$. Furthermore, for the impact of droplets in the film boiling regime, we measure the spreading factor and compare our data with existing models.

\subsection{Experimental Methods}

\subsubsection{Synthesis of carbon nanofiber layers}

Carbon nanofibers (CNFs) were synthesized on oxidized silicon wafers ( $p$ type, 5-10 Ohm.cm resistivity, $100 \mathrm{~mm}$ diameter, $525 \pm 25 \mu \mathrm{m}$ thickness, $\{100\}$ 
crystal orientation; Okmetic Finland) using a nickel (Ni) thin film as the catalyst. First, a $\mathrm{SiO}_{2}$ layer of $220 \mathrm{~nm}$ thickness was grown via wet oxidation (45 min, $1000{ }^{\circ} \mathrm{C}$ ) on these silicon substrates. Second, a pattern was defined in the spin-coated photoresist (Olin, 906-12), resulting in unmasked squares of $8 \mathrm{~mm} \times 8 \mathrm{~mm}$, by means of standard UV lithography (EVG 620). Furthermore, a $10 \mathrm{~nm}$ tantalum layer followed by a $25 \mathrm{~nm}$ nickel layer was deposited via electron-beam evaporation. Finally the samples were subjected to an ultrasonic lift-off step in acetone ( $>20 \mathrm{~min}$; VLSI 100038, BASF), followed by rinsing in water and spin drying. These nickelcoated substrates were diced into $1 \mathrm{~cm} \times 1 \mathrm{~cm}$ samples (Disco DAD-321 dicing machine). To remove organic contaminants, these samples were ultrasonically cleaned in acetone (10 min, Branson 200 ultrasonic cleaner) and de-ionized water (2 $\mathrm{min}$, $\left.25^{\circ} \mathrm{C}\right) .^{[29]}$

After drying with synthetic air, the samples were placed centrally on a flat quartz boat inside a quartz reactor and were loaded into a horizontal oven equipped with three temperature controllers along it. Nitrogen $\left(\mathrm{N}_{2} ; 99.999 \%\right.$, INDUGAS NV.) was used as a carrier gas during heating, pretreatment, CNF synthesis and cooling. First, the temperature was increased $\left(5 \mathrm{~K} . \mathrm{min}^{-1}\right)$ to $500{ }^{\circ} \mathrm{C}$. Second, the samples were pretreated with 20 vol.\% of hydrogen $\left(\mathrm{H}_{2} ; 99.999 \%\right.$, INDUGAS NV.) for 2 hours at a total flow rate of $50 \mathrm{ml} \cdot \mathrm{min}^{-1}$ in order to reduce the passivated $\mathrm{Ni}$ thin film. Subsequently the temperature was increased $\left(5 \mathrm{~K} \cdot \mathrm{min}^{-1}\right)$ to $635{ }^{\circ} \mathrm{C}$, at which temperature the CNF synthesis was performed via catalytic vapor decomposition using 25 vol. \% ethylene $\left(\mathrm{C}_{2} \mathrm{H}_{4} ; 99.95 \%\right.$ Praxair Inc.) and 6.25 vol.\% $\mathrm{H}_{2}$ at a total flow rate of $100 \mathrm{ml} \cdot \mathrm{min}^{-1}$. Finally the samples were cooled down to room temperature $\left(10 \mathrm{~K} \cdot \mathrm{min}^{-1}\right)$.

Two sets of samples were used for the droplet impact studies. One set was obtained after a CNF synthesis time of $11 \mathrm{~min}$, resulting in a CNF layer thickness $l \approx$ $3.4 \pm 0.3 \mu \mathrm{m}$. The other set was obtained after a CNF synthesis time of $14 \mathrm{~min}$, resulting in a CNF layer thickness of $l \approx 7.5 \pm 0.7 \mu \mathrm{m}$. More details of the influence of the synthesis time on the CNF layer thickness have been reported previously. ${ }^{[29]}$ These samples will be termed as $\operatorname{CNF}(3.5)$ and $\operatorname{CNF}(7.5)$, respectively. Figure 1 shows representative scanning electron microscopy (SEM) images with the side views 
(Figures 1a and b) and top views (Figures 1c and d) of the surfaces CNF(3.5) and $\operatorname{CNF}(7.5)$.

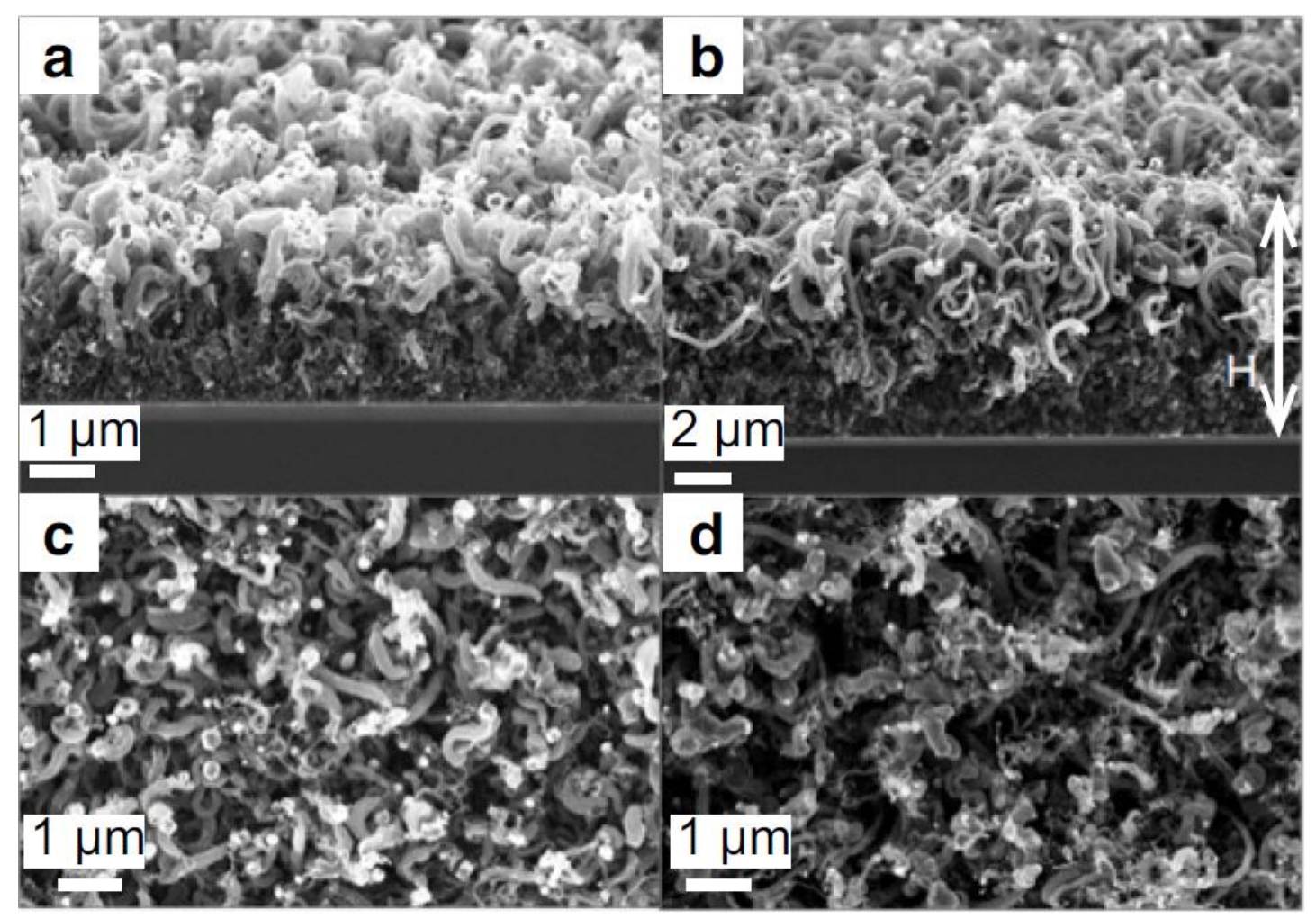

Figure 1: Scanning electron microscopy (SEM) images showing side views of the carbon nanofiber (CNF) layers with a synthesis time of (a) $11 \mathrm{~min}$ and (b) $14 \mathrm{~min}$. The arrow in (b) indicates the height $\boldsymbol{H}$ of the CNF layer. The corresponding top-view SEM-images are shown in (c) for a synthesis time of $14 \mathrm{~min}$. The bar represents $1 \mu \mathrm{m}$ in (a), (c) and (d), and $2 \mu \mathrm{m}$ in (b).

The thickness $l$ of the CNF layers was determined using 5 representative cross-sectional SEM images taken at various positions on the sample (10 height measurements were averaged per SEM image). The width of the nanofibers ranges from $32 \mathrm{~nm}$ to $220 \mathrm{~nm}$ with an average value of $127 \mathrm{~nm}$. Using the thickness of the CNF layers, the projected surface area of the samples $\left(A=0.64 \mathrm{~cm}^{2}\right)$, the density of graphite $\left(\rho=2267 \mathrm{~kg} . \mathrm{m}^{-3}\right)$, together with the total weight $M_{t}$ of all the carbon nanofibers on each sample $\left(M_{t}=120 \mu \mathrm{g}\right.$ for $\mathrm{CNF}(3.5)$ and $M_{t}=190 \mu \mathrm{g}$ for $\left.\mathrm{CNF}(7.5)\right)$, we can estimate the porosity $\emptyset=1-M_{t} /\left(\rho_{n} l A\right)$ of each sample: $\emptyset \approx 0.76$ and $\emptyset \approx 0.83$ for $\mathrm{CNF}(3.5)$ and $\mathrm{CNF}(7.5)$ respectively. 


\subsubsection{FC-72 droplet impact experiments on CNF layers}

A schematic diagram of the experimental setup is shown in Figure 2.

a

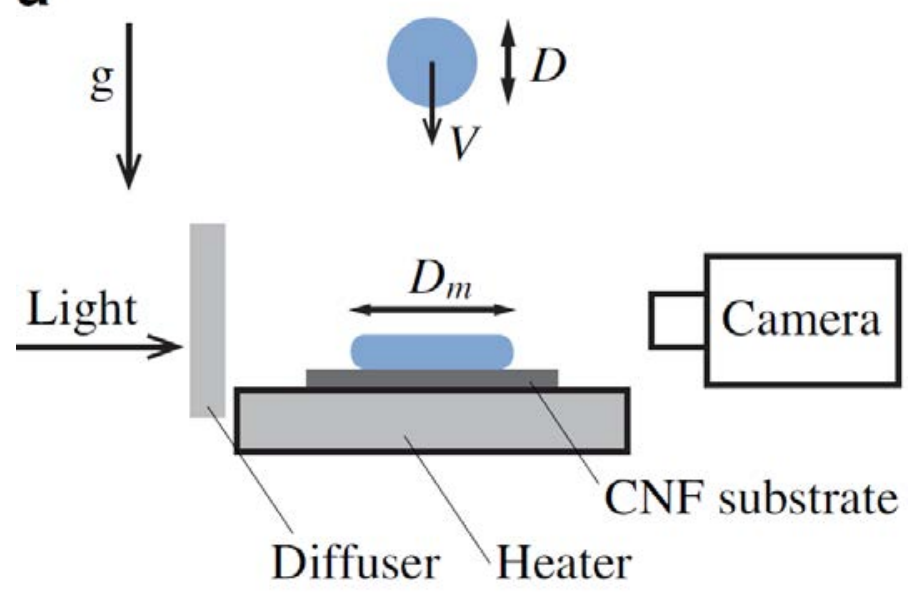

b
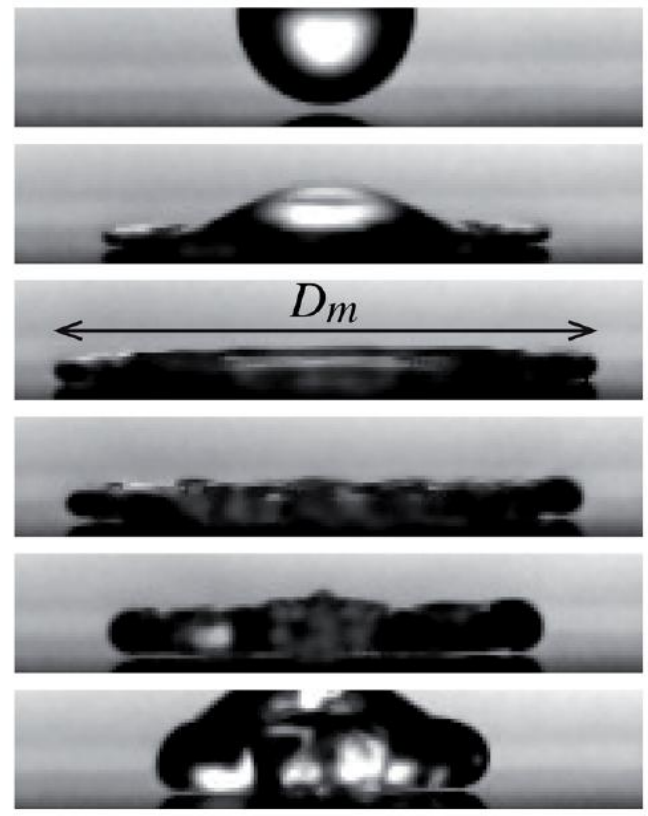

Figure 2: (a) Schematic (not to scale) of the experimental setup used to observe the characteristic behaviors of impacting droplets on heated surfaces. The surface of interest is placed on a heater, which can be heated up to $500{ }^{\circ} \mathrm{C}$. FC-72 droplets of diameter $D$ impact the heated sample with the impact velocity $V$. The behavior of the impacting droplets is recorded from the side using a high-speed camera (Photron SA1.1). From the recordings, $D, V$, and the maximum spreading $D_{m}$ of the droplet can be measured. (b) Series of snapshots of an impacting droplet in the film boiling regime showing how $D_{m}$ is measured as the maximum horizontal extension of the droplet. 
All droplet impact experiments were performed with FC-72 (3M Fluorinert Electronic Liquid), a dielectric fluid commonly used in electronics cooling applications. The liquid has a boiling point $T_{b}=56^{\circ} \mathrm{C}$, a density $\rho_{l}=1680 \mathrm{~kg} \cdot \mathrm{m}^{-3}$, and a surface tension $\sigma=10 \mathrm{mN} \cdot \mathrm{m}^{-1}$. We generate droplets by using a syringe pump (PHD 2000 Infusion, Harvard Apparatus) to inject the liquid into a small fused silica needle where the droplets are formed at the tip. The flow rate is kept at a small value $\left(\approx 0.1 \mathrm{~mL} \cdot \mathrm{min}^{-1}\right)$ so that droplet detachment from the needle is due only to the gravitational force, hence keeping the droplet size uniform. After detaching from the needle, a droplet falls on the target surface placed on a brass plate with a cartridge heater and a thermocouple (Omega Inc.) embedded inside. The surface temperature $T$ was set using a controller and was varied between $60^{\circ} \mathrm{C}$ and $450{ }^{\circ} \mathrm{C}$. This temperature was also measured independently using a surface temperature probe (Tempcontrol B.V.). The difference between the controller's set point and the surface probe measurement was less than $3 \mathrm{~K}$. Thus we take the controller's set point as the surface temperature $T$.

Recordings of the impact events were made with a high-speed camera (Photron SA1.1) (see Figure 2). From these high-speed recordings, the boiling behaviors were analyzed, and the droplet diameter $D$, the impact velocity $V$ and the maximum spreading diameter $D_{m}$ (see Figure 2) were measured. From the measured diameter and velocity, we calculated the Weber number $W e=\rho_{l} D V^{2} / \sigma$, which is a dimensionless number that characterizes the droplet's kinetic energy compared to its surface energy. The impact velocity $V$ was varied by changing the needle's height. Impact events were repeated at least three times for every combination of $V$ and $T$ to test the reproducibility of the experiment.

\subsubsection{Characterization of boiling behavior}

By varying the surface temperature between $60{ }^{\circ} \mathrm{C}$ and $450{ }^{\circ} \mathrm{C}$ and the Weber number between 10 and 1000, we observed two characteristic boiling behaviors: contact boiling and film boiling. In Figure 3 we show two series of images to illustrate the difference between these two regimes. The essential difference between the two is whether or not the liquid makes direct contact with the heated surface during impact. ${ }^{[21,30,31]}$ 
a

Contact boiling $\left(200^{\circ} \mathrm{C}\right)$
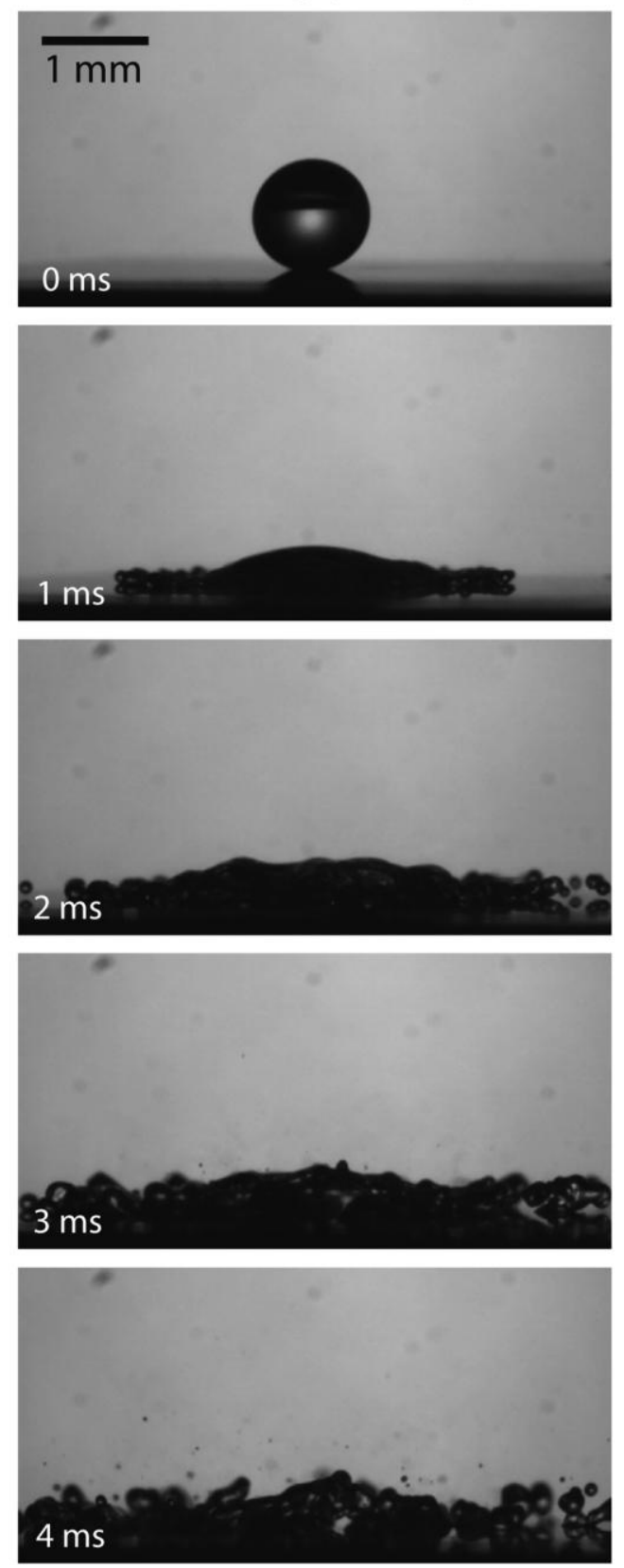

b

\section{Film boiling $\left(400^{\circ} \mathrm{C}\right)$}
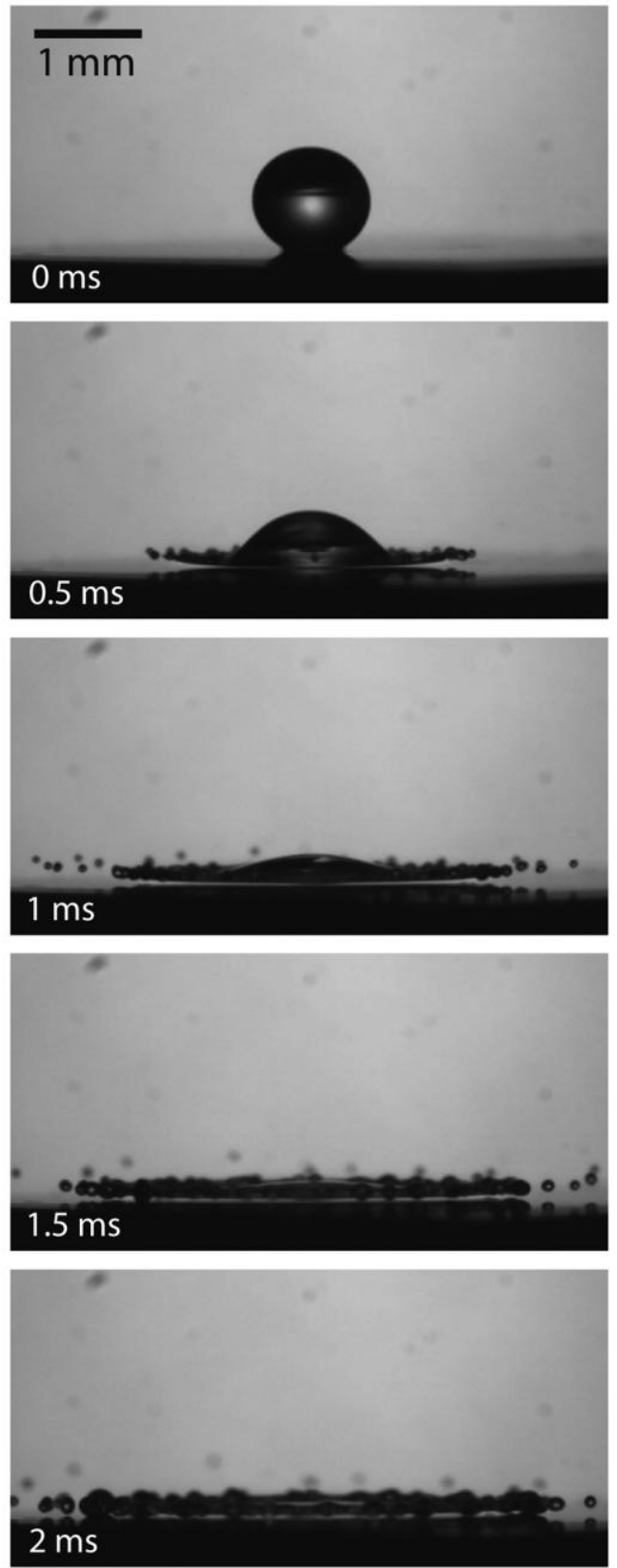

Figure 3: (a) Representative images showing the characteristic boiling behavior of an impacting FC-72 droplet on a $7.5 \mu \mathrm{m}$-thick $\mathrm{CNF}$ surface in the contact boiling regime, $\mathrm{T}=200{ }^{\circ} \mathrm{C}$. The diameter of the impacting droplet is $D=1.1 \mathrm{~mm}$, the impact velocity $V=1.0 \mathrm{~m} . \mathrm{s}^{-1}$ and the Weber number $W e=154$. (b) Representative images of an FC-72 droplet with the same diameter and velocity impacting on the same surface as in (a), but at the higher surface temperature, $T=400$ ${ }^{\circ} \mathrm{C}$. In this case, the impact is in the film boiling regime. 
In the contact boiling regime (Figure 3a), as the pressure of the vapor generated underneath the droplet is not sufficient to support the droplet's dynamic pressure, the liquid touches the heated surface and quickly boils due to the high heat flux through the contact area. The recorded snapshots show the small droplets ejected as a result of the boiling process. In contrast, an impacting droplet in the film boiling regime is separated from the heated surface by a developing vapor layer (see Figure 3b). This vapor layer insulates the droplet during the impact time, and hence prevents the liquid from boiling violently.

By carefully analyzing the recorded movies of impacting droplets, we categorized the impact as being in the film boiling regime when droplet ejection or vapor bubble generation was not observed.

\subsection{Results and Discussions}

\subsubsection{Dynamic Leidenfrost temperature}

In Figure 4 we show phase diagrams of the characteristic boiling behavior of impacting droplets on smooth silicon surfaces, $\mathrm{CNF}(3.5)$, and $\mathrm{CNF}(7.5)$, respectively. The temperature ranges were $60{ }^{\circ} \mathrm{C}$ to $250{ }^{\circ} \mathrm{C}$ for the smooth silicon surfaces, $60{ }^{\circ} \mathrm{C}$ to $300{ }^{\circ} \mathrm{C}$ for the $\mathrm{CNF}(3.5)$ surfaces, and $100{ }^{\circ} \mathrm{C}$ to $450{ }^{\circ} \mathrm{C}$ for the $\mathrm{CNF}(7.5)$ surfaces. In each phase diagram, there is a clear transition between the contact and the film boiling regimes. This transition temperature is marked by a solid line, with the vertical bars indicating the intermediate region where both boiling behaviors were observed. The transition temperature, known as the dynamic Leidenfrost temperature $T_{L}$, increases with increasing kinetic energy of impacting droplets. This dependence of $T_{L}$ on $W e$ is qualitatively similar to that found previously for droplets impacting on smooth and micro-structured surfaces, ${ }^{[15,16,21]}$ and is expected: the increasing momentum of the impact forces the droplet to contact with the surface at higher and higher temperatures.

These results, however, are in stark contrast to those found for smooth and microstructured surfaces in two respects, as can be seen from Figure 5 in which $T_{L}$ values for the smooth and CNF surfaces are compared. 
a

Smooth silicon

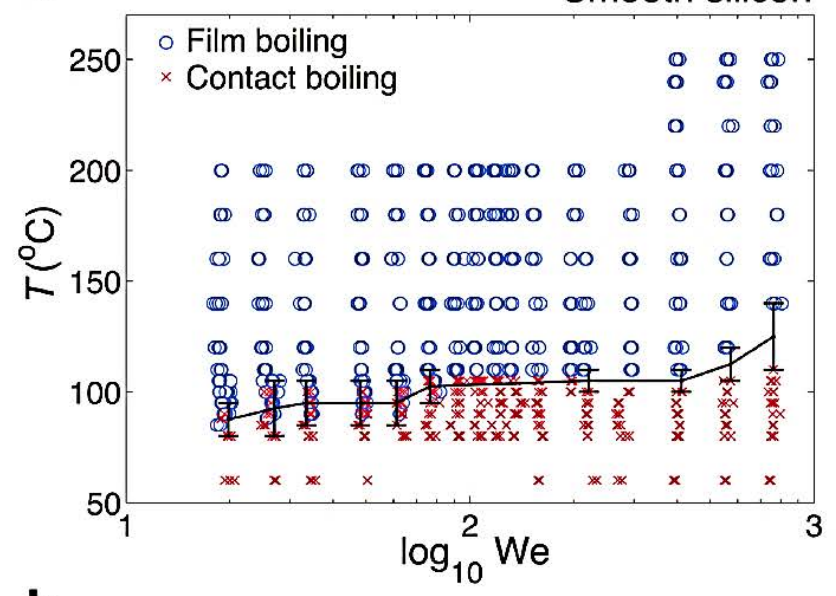

b

$\operatorname{CNF}(3.5)$
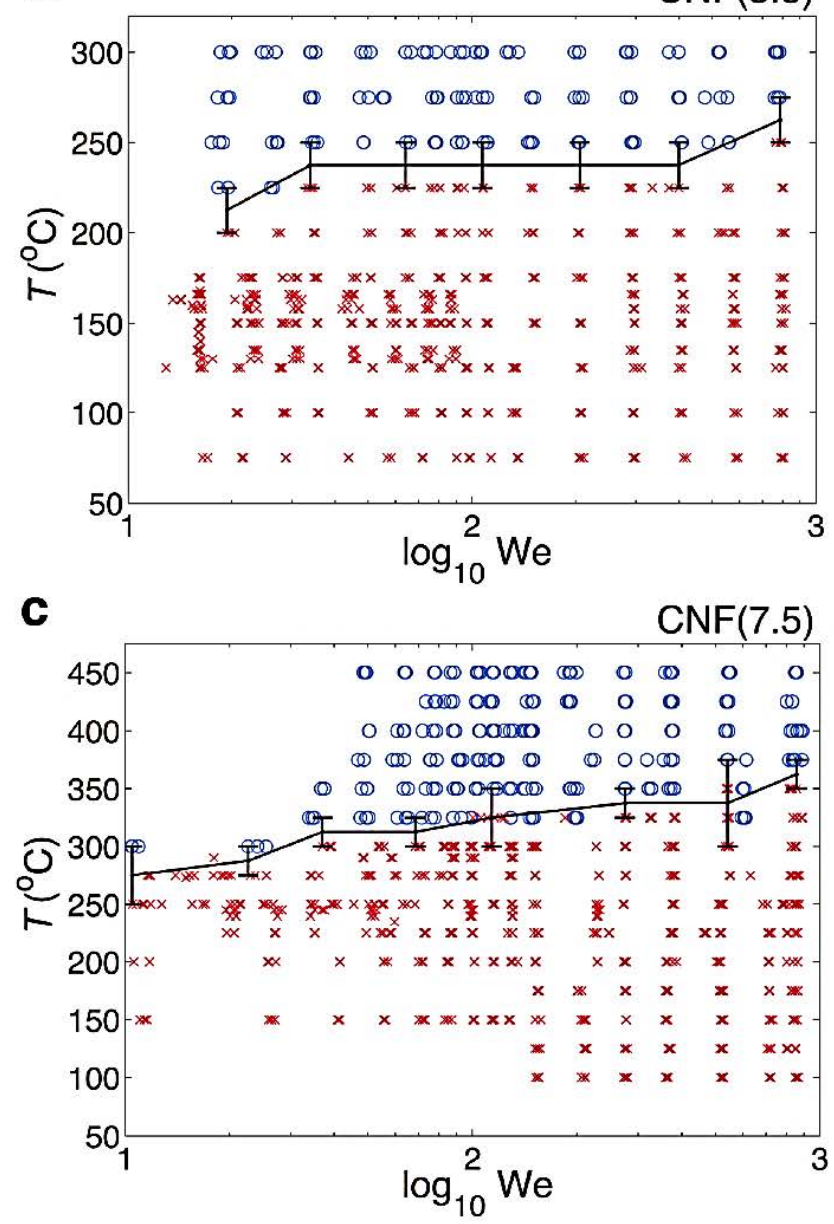

Figure 4: (a) Phase diagram showing the characteristic boiling behaviors of impacting FC-72 droplets on smooth silicon surfaces. The contact boiling regime (red crosses) and film boiling regime (open blue circles) are separated by a transition band, indicated by the vertical bars, where both characteristic behaviors were observed. (b) Phase diagram for surfaces covered with a $3.5 \mu \mathrm{m}$-thick CNF layer. (c) Phase diagram for surfaces covered with a $7.5 \mu \mathrm{m}$-thick CNF layer. Note the much larger temperature ranges in (b) and (c). 


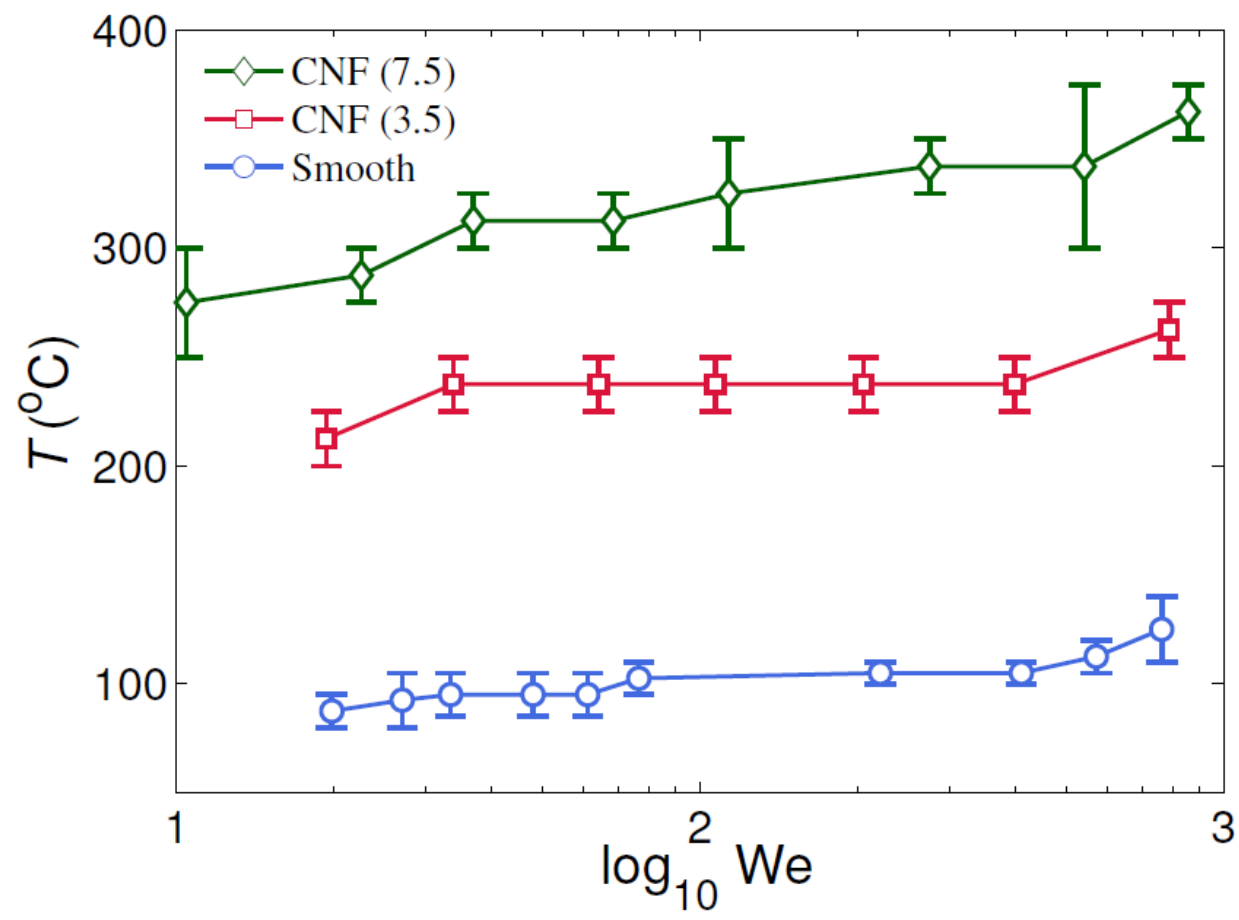

Figure 5: Dynamic Leidenfrost temperature (transition from contact to film boiling) for smooth silicon, and surfaces covered with a $3.5 \mu \mathrm{m}$ and a $7.5 \mu \mathrm{m}$-thick layer of carbon nanofibers.

The first unexpected finding is that while $T_{L}$ is lower for microstructured surfaces as compared with smooth ones, ${ }^{[16]}$ it is actually higher in the case of carbon nanofibers. For example, for $W e=100, T_{L}$ for the smooth surface is $110^{\circ} \mathrm{C}$, whereas for $\mathrm{CNF}(3.5)$ and $\mathrm{CNF}(7.5)$ it increases to $250^{\circ} \mathrm{C}$ and $350{ }^{\circ} \mathrm{C}$, respectively. Secondly, $T_{L}$ increases with the nanofiber length, again in contrast to surfaces covered with micrometer-sized pillars for which, for given shape and spacing, the microstructure height is inversely correlated with $T_{L}{ }^{[16]}$ The tentative explanation of that latter finding offered in Ref. 16 is that the surface of the impacting liquid tends to penetrate the space between the pillars. This causes the liquid-surface area to increase, the more the higher the pillars. As a consequence, the vapor generation rate also increases and the film boiling regime sets in at a lower temperature.

As an explanation of the opposite behavior found with carbon nanofibers we suggest that they are efficiently cooled by the vapor flow before the drop touches the CNF surface. To support this conjecture in the following subsection we will estimate the time scale $\tau_{c}$ for the temperature of the nanofibers to cool, and compare it with the time scale $\tau_{e}$ the nanofiber is exposed to the vapor flow (which will be found to be 
somewhat larger), and also with the time scale $\tau_{h}$ for the heat flow inside the nanofiber (which will be found to be much larger).

\subsubsection{Estimate of the relevant time scales}

We start with the estimate for the time scale $\tau_{c}$ for the cooling of the nanofibers by the "vapor wind". Since the cross-section of the nanofibers is of the order of $100 \mathrm{~nm}$, the time scale $\tau_{c}$ can be estimated by assuming their temperature to be uniform, which is the so-called lumped capacitance approximation. ${ }^{[32]}$ This time scale can then be estimated to be

$$
\tau_{c} \sim \frac{w \rho_{n} C_{n}}{h}
$$

in which $w$ is the diameter of the nanofiber, $\rho_{n}$ and $C_{n}$ are its density and specific heat, and $h$ is the heat transfer coefficient. The latter can be expressed in terms of a Nusselt number,

$$
N u \sim \frac{w h}{K_{v}}
$$

with $K_{v}$ being the vapor thermal conductivity, so that

$$
\tau_{c}=\frac{\rho_{n} C_{n} w^{2}}{K_{V} N u}=\frac{\rho_{n} C_{n}}{\rho_{v} C_{v}} \frac{w^{2}}{k_{v} N u}
$$

in which $\rho_{v}, C_{v}$, and $k_{v}$ are the vapor density, specific heat, and thermal diffusivity, respectively. The (approximate) proportionality of $\tau_{c}$ to the square of the fiber size is a particularly noteworthy feature of this expression. In standard correlations, ${ }^{[32]} \mathrm{Nu}$ is given as a function of the Prandtl and Reynolds numbers. No measured value for the former seems to be available for FC-72 vapor, but it is well known that the Prandtl number of gases is close to 1 and we can safely use this estimate here. Estimation of the Reynolds number requires a value for the viscosity of the vapor which, again, does not seem to have been measured. The order of magnitude of the viscosity of many gases and vapors is $10^{-5}$ Pa.s, and this is the value we will use. The density of FC-72 vapor at the boiling point of $56^{\circ} \mathrm{C}$ is about $11.5 \mathrm{~kg} \cdot \mathrm{m}^{-3}$. Taking $w \sim 100 \mathrm{~nm}$ and using these values we then find $\operatorname{Re} \sim 0.1 V_{v}$, with $V_{v}$ being the vapor velocity in $\mathrm{m} . \mathrm{s}^{-1}$. This quantity has been estimated in Ref. 16 (see equation (13) of that paper) where it was found to be of the order of 


$$
V_{v} \sim\left(\frac{\rho_{l} C_{v} \Delta T}{\rho_{v} L P r_{v}}\right)^{1 / 2} V
$$

with $\rho_{l}$ being the liquid density, $\Delta T$ being the liquid-surface temperature difference, $L$ being the latent heat and $P r_{v}$ being the vapor Prandtl number. With $\rho_{l}=1680 \mathrm{~kg} \cdot \mathrm{m}^{-3}$, $L=88 \mathrm{~kJ} \cdot \mathrm{kg}^{-1}, \mathrm{C}_{\mathrm{v}}=910 \mathrm{~J} \cdot \mathrm{kg}^{-1} \cdot \mathrm{K}^{-1}$ and $\rho_{v}=11.5 \mathrm{~kg} \cdot \mathrm{m}^{-3}$ (values at $56^{\circ} \mathrm{C}$ ), $\Delta T \sim 100 \mathrm{~K}$, the impact velocity $V \sim 1 \mathrm{~m} \cdot \mathrm{s}^{-1}$ and again taking $\operatorname{Pr}_{v} \sim 1$, we find $V_{v} \sim$

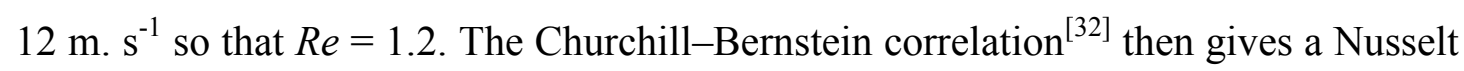
number of about 1. The use of Equation (2) requires values of $K_{v}$ or $k_{v}$, neither of which seems to be available. For many gases and vapors $k_{v}$ is of the order of $10^{-5} \mathrm{~m}^{2} . \mathrm{s}^{-1}$. With this estimate, taking $\rho_{n} \approx 2267 \mathrm{~kg} \cdot \mathrm{m}^{-3}, C_{n} \approx 709 \mathrm{~J} \cdot \mathrm{kg}^{-1} \cdot \mathrm{K}^{-1}$ and, again, $w \sim 100 \mathrm{~nm}$, we find from Equation (2) $\tau_{c} 150 \mathrm{~ns}$.

This time scale has to be compared with the characteristic time $\tau_{e}$ during which the fiber is exposed to the cooler vapor until the liquid makes contact with it, which can be estimated as

$$
\tau_{e} \sim \frac{\mathrm{H}_{v}}{V}
$$

where $H_{v} \sim D S t^{-2 / 3}$ is the characteristic thickness of the vapor layer at which the drop starts being deformed due to the increasing pressure on its underside. ${ }^{[33]}$ Here, as above, $D$ is the droplet diameter, $V$ is the impact velocity, and $S t=\rho_{l} V D / \mu_{v}$ is the Stokes number, where $\mu_{v}$ is the viscosity of vapor. Hence we obtain the time during which the nanofibers are exposed to the cooler vapor flow $\tau_{e} \sim D S t^{-2 / 3} / V$. In the use of this estimate we again encounter the problem that $\mu_{v}$ is not available but, if we use the same estimate $\mu_{v} \sim 10^{-5} \mathrm{~Pa}$.s as before and take $V \approx 1 \mathrm{~m} \cdot \mathrm{s}^{-1}, D \approx 1 \mathrm{~mm}$, we find $\tau_{e} 330 \mathrm{~ns}$, which is seen to be long enough to cause a substantial cooling of the fibers.

Of course, as the fibers are cooled by the vapor, heat flows towards their tips from the silicon substrate with a characteristic time

$$
\tau_{h} \sim \frac{l^{2}}{k_{c}}
$$

in which $l$ is the fiber length and $k_{c}$ is the thermal diffusivity of the carbon nanofibers. Since, in this experiment, the fibers had not been heat-treated, we can estimate their 
thermal conductivity on the basis of the results of ref. 34 as $K_{c}=4.6 \mathrm{~W} \cdot \mathrm{m}^{-1} \cdot \mathrm{K}^{-1}$ and, therefore, $k_{c} \sim 2.86 \times 10^{-6} \mathrm{~m}^{2} . \mathrm{s}^{-1}$. For the shorter fibers $l \approx 3.4 \mu \mathrm{m}$ and, therefore, $\tau_{h} \sim 4 \mu \mathrm{s}$, while for the longer fibers, $l \approx 7.5 \mu \mathrm{m}$ and $\tau_{h} \sim 20 \mu \mathrm{s}$. These times are much longer than both the cooling time and the exposure time to the vapor flow, which implies that the liquid encounters fibers at a much cooler temperature than the core silicon substrate. This circumstance would explain why the CNF surfaces require a higher temperature to achieve the film boiling regime compared to the smooth surfaces, and why the transition temperature increases with the fiber length. The size of the cross-section of the fibers in our experiment is close to the cross-over value at which cooling and exposure to the vapor flow have comparable time scales. It follows that fibers or, more generally, microstructures with a larger cross-section would be insensitive to the cooling effect. As a check of this expectation we can apply the same estimates to the case of the microstructured surfaces studied earlier. ${ }^{[16]}$ In that case the fluid was water for which, of course, all the required physical properties are well known. The microstructures had the form of silicon pillars with a square cross-section of about $10 \times 10 \mu \mathrm{m}^{2}$ and heights from 2 to $8 \mu \mathrm{m}$. The vapor velocity estimated from Equation (3), again with $\Delta T \sim 100 \mathrm{~K}$ and $V \sim 1 \mathrm{~m} . \mathrm{s}^{-1}$, is found to be $V_{v} \sim 12 \mathrm{~m} . \mathrm{s}^{-1}$.

The corresponding Reynolds number is $R e \sim 6$ with the corresponding Nusselt number $N u \sim 1.7$. In this case $\rho_{n}=2330 \mathrm{~kg} \cdot \mathrm{m}^{-3}, C_{n}=705 \mathrm{~J} \cdot \mathrm{kg}^{-1} \cdot \mathrm{K}^{-1}$ and Equation (2) gives $\tau_{c} \sim 6.6 \mathrm{~ms}$. The exposure time to the vapor is not very different from the previous estimate, and is therefore several orders of magnitude shorter. It is evident that, in this case, the vapor flow is just a small perturbation which does not have an appreciable effect on the pillar temperature.

\subsubsection{Spreading factor}

We devote this section to quantifying the spreading factor of impacting droplets in the film boiling regime. The spreading factor is defined as $D_{m} / D$, where $D_{m}$ is the maximum spreading diameter. In Figure 6, we show a $\log -\log$ plot of $D_{m} / D$ versus $W e$ for all the impact experiments obtained on smooth and CNF surfaces. All the data points were collected for impacts in the film boiling regime and in the course of which the droplets did not disintegrate during the expanding phase. The Weber number ranges from 5 to 600 . 


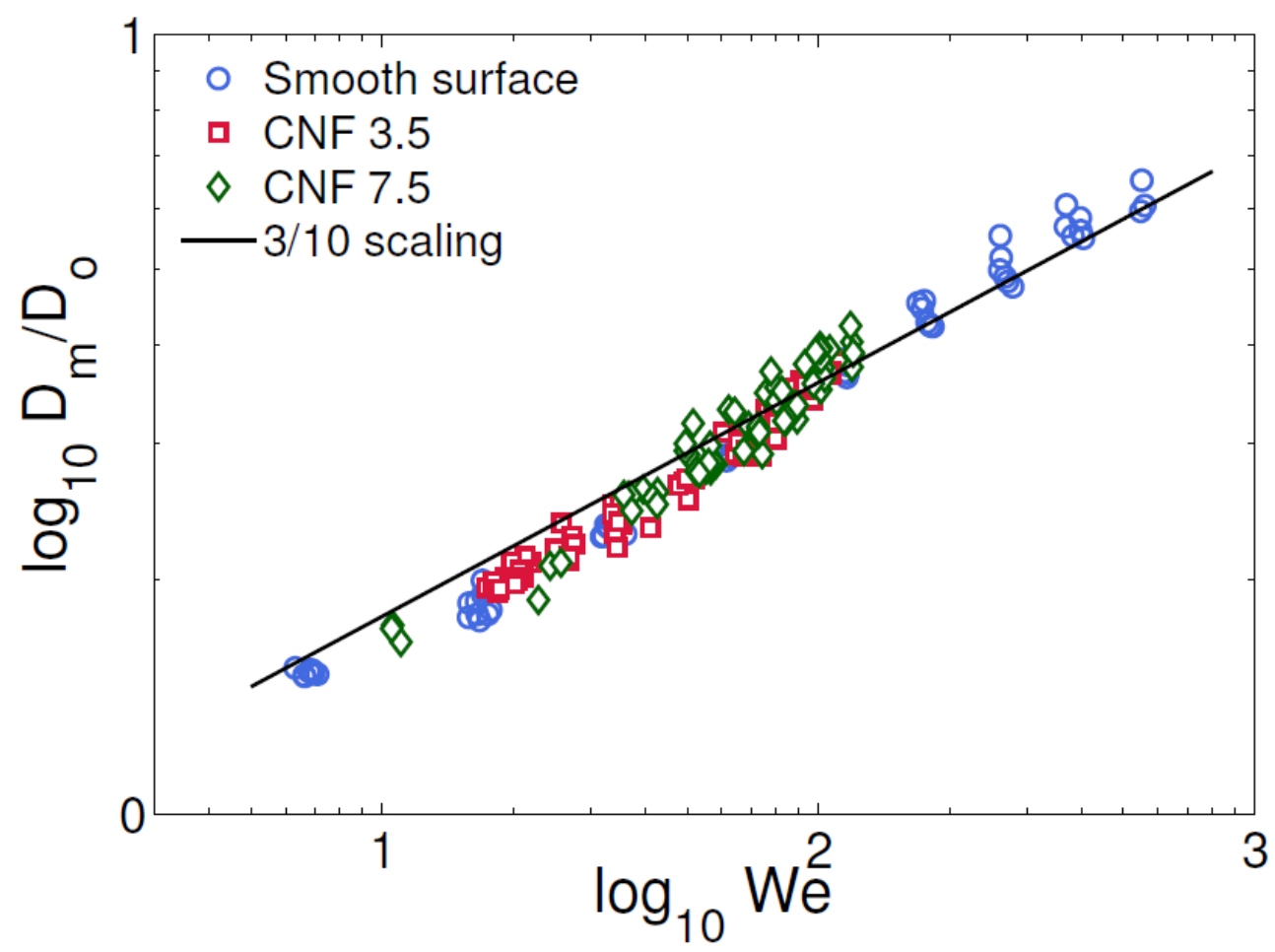

Figure 6: Spreading factor $D_{m} / D$ for impacting FC-72 droplets on three surfaces: smooth silicon, and silicon covered with a $3.5 \mu \mathrm{m}$ and a $7.5 \mu \mathrm{m}$-thick layer of carbon nanofibers. All the data points were obtained for impacts in the film boiling regime for which the impacting drop did not fragment in smaller droplets. The solid line represents the scaling relationship $D_{m} / D \sim W e^{3 / 10}$ derived by taking the vapor flow as the major driving mechanism for the spreading of the liquid. ${ }^{[16]}$

All datasets collected from the three different surfaces collapse on the same curve, showing that the spreading dynamics does not depend on the features and temperature of the surfaces. This result is consistent with the recent study of impacting droplets on micro-structured surfaces, ${ }^{[16]}$ which showed that the spreading factor is independent of the microstructures and depends very weakly on the surface temperature. Moreover, the spreading factor for $\mathrm{We}>80$ is in agreement with the scaling $D_{m} / D \propto W e^{3 / 10}$ derived in Ref. 16 for the large Weber number regime. This scaling law embodies the main assumption that the spreading of the liquid is driven by the vapor flow underneath the droplet. ${ }^{[16]}$ Note that other studies have experimentally found that for $\mathrm{We}<100$, a scaling exponent $\approx 0.4$ can be used to describe the impact of droplets on different types of surfaces, e.g., on hydrophobic powders, ${ }^{[35]}$ and on sublimating surfaces. ${ }^{[36]}$ However, due to different ranges of the Weber number, these 
studies do not contradict our results of the spreading factor. As a result, we conclude that the presence of the carbon nanofibers only changes the transition temperature to film boiling of the impacting droplets, but does not affect the dynamics of the vapor flow in the film boiling regime or the liquid spreading.

\subsection{Conclusions}

We have explored the phase space ( $W e, T)$ of the impact of FC-72 droplets on heated smooth silicon surfaces and surfaces coated with nanofibers (CNFs) of different lengths. Unexpectedly, we have found that the dynamic Leidenfrost temperature $T_{L}$, i.e., the transition temperature between the contact and film boiling regimes, is higher on the CNF surfaces than on the smooth silicon surface. Increasing the fiber length from $3.5 \mu \mathrm{m}$ to $7.5 \mu \mathrm{m}$ causes $T_{L}$ to increase significantly due to the small time scale with which the nanofibers cool to the temperature of the vapor generated by the approaching liquid. Thus, the temperature of the fibers when in contact with the liquid is established is much lower than their initial temperature. In other words, the temperature of the CNF surfaces has to be set higher than in the case of smooth silicon surfaces to bring the impact in the film boiling regime. In contrast, the silicon microstructured surfaces studied in Ref. 16 maintain their temperature during impact and $T_{L}$ is lower, possibly because the liquid surface area which generates the vapor is larger due to the curvature caused by the micro-pillars.

In spite of the effect on $T_{L}$, we have found that as long as the impact is in the film boiling regime, the spreading factor of the droplet does not depend on whether the surface is smooth or covered with carbon nanofibers, nor does it depend on the surface temperature. The spreading factor is consistent with the scaling law $D_{m} / D \propto W e^{3 / 10}$, which was derived based on the effect of vapor flow on the spreading dynamics. ${ }^{[16]}$

The increase in the dynamic Leidenfrost temperature caused by nanofibers fabricated on silicon surfaces has a considerable implication for various applications that require high operating temperatures because CNF surfaces can operate at higher $T_{L}$ while still maintaining contact with the liquid. 


\subsection{References}

[01] J. Kim, Int. J. Heat Fluid Flow, 2007, 28, 753.

[02] B. Agostini, M. Fabbri, J. E. Park, L. Wojtan, J. R. Thome, and B. Michel, Heat Transfer Eng., 2007, 28, 258.

[03] M. A. Ebadian and C. X. Lin, J. Heat Transfer, 2011, 133, 110801.

[04] A. G. Pautsch and T. A. Shedd, Int. J. Heat Mass Transfer, 2005, 48, 3167.

[05] M. Visaria and I. Mudawar, IEEE Trans. Compon., Packag., Manuf. Technol., 2009, 32, 784.

[06] C. Arcoumanis, P. Cutter, and D. S. Whitelaw, Chem. Eng. Res. Des., 1998, 76, 124.

[07] M. R. O. Panao and A. L. N. Moreira, Int. J. Therm. Sci., 2009, 48, 1853.

[08] N. Mascarenhas and I. Mudawar, Int. J. Heat Mass Transfer, 2012, 55, 2953.

[09] S. S. Yoon, V. Figueroa, A. L. Brown, and T. K. Blanchat, J. Fire Sci., 2010, 28, 109.

[10] P.-P. Chen and X.-S. Wang, Int. J. Heat Mass Transfer, 2011, 54, 4143.

[11] A. L. N. Moreira, A. S. Moita, and M. R. Paña, Prog. Energy Combust. Sci., 2010, 36, 554.

[12] E. Berberovic, I. V. Roisman, S. Jakirlic, and C. Tropea, Int. J. Heat Fluid Flow, $2011,32,785$.

[13] A. Yarin, Annu. Rev. Fluid Mech., 2006, 38, 159.

[14] S. Herbert, T. Gambaryan-Roisman, and P. Stephan, Colloids Surf., A, 2013, 432, 57.

[15] J. D. Bernardin, C. J. Stebbins, and I. Mudawar, Int. J. Heat Mass Transfer, 1997, 40, 247.

[16] T. Tran, H. J. J. Staat, A. Susarrey-Arce, T. C. Foertsch, A. van Houselt, J. G. E. Gardeniers, A. Prosperetti, D. Lohse, and C. Sun, Soft Matter, 2013, 9, 3272.

[17] J. D. Bernardin and I. Mudawar, J. Heat Transfer, 1999, 121, 894.

[18] A. B. Wang, C. H. Lin, and C. C. Chen, Phys. Fluids, 2000, 12, 1622.

[19] J. D. Bernardin and I. Mudawar, J. Heat Transfer, 2004, 126, 272. 
[20] I. U. Vakarelski, N. A. Patankar, J. O. Marston, D. Y. C. Chan, and S. T. Thoroddsen, Nature, 2012, 489, 274.

[21] T. Tran, H. J. J. Staat, A. Prosperetti, C. Sun, and D. Lohse, Phys. Rev. Lett., 2012, 108, 036101.

[22] J. Lee, J. Kim, and K. Kiger, Int. J. Heat Fluid Flow, 2001, 22, 188.

[23] C. M. Weickgenannt, Y. Zhang, S. Sinha-Ray, I. V. Roisman, T. GambaryanRoisman, C. Tropea, and A. L. Yarin, Phys. Rev. E: Stat., Nonlinear, Soft Matter Phys., 2011, 84, 036310.

[24] C. M. Weickgenannt, Y. Zhang, A. N. Lembach, I. V. Roisman, T. GambaryanRoisman, A. L. Yarin, and C. Tropea, Phys. Rev. E: Stat., Nonlinear, Soft Matter Phys., 2011, 83, 036305.

[25] R. H. Chen, S. L. Chiu, and T. H. Lin, Appl. Therm. Eng., 2007, 27, 2079.

[26] R.-H. Chen, S.-L. Chiu, and T.-H. Lin, Exp. Therm. Fluid Sci., 2007, 32, 587.

[27] S. Jun, S. Sinha-Ray, and A. L. Yarin, Int. J. Heat Mass Transfer, 2013, 62, 99.

[28] J. H. Bitter, J. Mater. Chem., 2010, 20, 7312.

[29] H. Nair, R. M. Tiggelaar, D. B. Thakur, J. G. E. Gardeniers, A. van Houselt, and L. Lefferts, Chem. Eng. J., 2013, 227, 56.

[30] H. Fujimoto, Y. Oku, T. Ogihara, and H. Takuda, Int. J. Multiphase Flow, 2010, 36, 620 .

[31] D. Quéré, Annu. Rev. Fluid Mech., 2013, 45, 197.

[32] F. P. Incropera, D. P. DeWitt, T. L. Bergman, and A. S. Lavine, Fundamentals of Heat and Mass Transfer, 7th edn, Wiley, 2010.

[33] S. Mandre, M. Mani, and M. P. Brenner, Phys. Rev. Lett., 2009, 102, 134502.

[34] E. Mayhew and V. Prakash, Carbon, 2013, 62, 493.

[35] J. O. Marston, Y. Zhu, I. U. Vakarelski, and S. T. Thoroddsen, Powder Technol., 2012, 228, 424.

[36] C. Antonini, I. Bernagozzi, S. Jung, D. Poulikakos, and M. Marengo, Phys. Rev. Lett., 2013, 111, 014501. 


\section{Chapter 8}

Summary and Outlook

Abstract

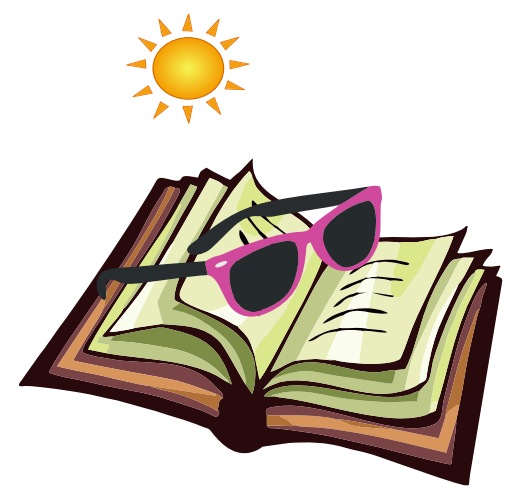

The subject of this thesis is the direct synthesis of well-adhesive carbon nanofiber (CNF) layers via thermal catalytic chemical vapor deposition. The aim is to grow spatially uniform CNF layers with controllable morphology and controllable wettability on Si based Ni thin film substrates. This thesis also discusses the evaporation of water droplets on this CNFs as well as the impact of droplets and the subsequent spreading behavior on heated CNF surfaces. The results of these investigations are summarized in this chapter and an outlook is formulated. 


\subsection{Summary}

The wettability of surfaces is determined by surface energy and roughness. In this thesis we study the growth and wetting properties of Carbon nanofibers (CNFs).

In Chapter 1 an introduction to the CNFs and wettability, the main subjects in this thesis, is provided. The study of CNFs is placed in a historic perspective and the growing interest in superhydrophobicity is shortly introduced.

In Chapter 2 the evaporation of a water droplet on FOTS-coated microtextured omniphobic silicon surface, fabricated by photolithography, is studied. The evaporation driven Cassie-Baxter to Wenzel wetting transition, which is earlier reported on superhydrophobic surfaces, is absent on our omniphobic surfaces. The added edge-curvature and roughness are shown to result in a delayed or totally absent Cassie-Baxter to Wenzel transition. A global interfacial energy argument, which can be successfully applied to predict the Cassie-Baxter to Wenzel transition for pillars with sharp edges and straight interfaces, fails to predict the transition on our omniphobic surfaces, probably as a consequence of the added energy barrier arising from the added edge-curvature and roughness.

Chapter 3 details the influence of the pretreatment on the dewetting of the $\mathrm{Ni}$ thin film prior to the CVD growth process of the CNFs. Various nickel-based thin films - $10 \mathrm{~nm} \mathrm{Ni}, 25 \mathrm{~nm} \mathrm{Ni}$ and $25 \mathrm{~nm} \mathrm{Ni} / 10 \mathrm{~nm} \mathrm{Ta}$ - on oxidized silicon supports were fabricated as support materials for the CNF synthesis and are subjected to different pretreatments (also pretreatment temperatures and pretreatment time lapses) in vacuum, air, nitrogen or hydrogen. For a pretreatment temperature of $650{ }^{\circ} \mathrm{C}(1 \mathrm{~h})$ in vacuum, air, nitrogen or hydrogen atmospheres only the latter results in the formation of separate nanoparticles. EDX-analysis shows that the particles are composed of Ni. No difference in Ni dewetting behavior is found between oxidized silicon and fused silica substrates. In case of reduced $25 \mathrm{~nm} \mathrm{Ni} / 10 \mathrm{~nm}$ Ta the diameter of the resulting nanoparticles has a sharp size distribution with an average of $56 \mathrm{~nm}$.

On $\mathrm{Ni}$ thin film samples pretreated in vacuum or nitrogen and exposed to the CNF synthesis procedure, amorphous carbon layers are formed on metallic thin films that are not completely dewetted into nanoparticles. Depending on the CNF growth 
time and the absence/presence of Ta, the carbonaceous layers are continuous (and conformal) or porous, resulting in hardly any or scarce growth of CNFs. In case of airpretreated Ni samples, severe release of CNF carpets from the substrate occurs for $\mathrm{CNF}$ synthesis times of at least $1 \mathrm{~min}$. It is concluded that pretreatment of $\mathrm{Ni}$ thin films in vacuum, nitrogen or air should not be performed when well-attached homogeneous layers of CNFs are desired. Good results are only obtained on reduced Ni films with an adhesion layer of Ta, giving homogeneously distributed, randomlyoriented, well-attached and semi-crystalline CNFs within 3 min synthesis time. The diameter of these fibers is in the range 15-40 nm, which shows good correlation with the particle size distribution after reduction. Absence of the adhesion layer results in the formation of nanoparticles of several hundreds of nanometers diameter on which an amorphous carbon layer is deposited but no CNFs grow for synthesis times up to 3 min. It is found that small spherical nanoparticles with a diameter below $80 \mathrm{~nm}$ are a necessity for fast initiation of CNF nucleation and growth. For the conditions explored in this work this is only the case for reduced $\left(650^{\circ} \mathrm{C}, 1 \mathrm{~h}\right)$ films composed of e-beam evaporated $\mathrm{Ni}(25 \mathrm{~nm})$ and $\mathrm{Ta}(10 \mathrm{~nm})$.

In an attempt to get more insight into the growth process and the nature of the synthesized CNF layers, the samples grown for varying growth time (0-60 min) were characterized in Chapter 4 by Raman and Infrared spectroscopy in addition to high resolution scanning electron microscopy (HRSEM) and Helium Ion Microscopy (HIM). The development of a thin "carpet" layer during the CNF growth on samples without a Ta adhesion layer was observed. This layer is assigned as a graphitic like carbon layer, which most probably develops via silicon carbide, which is formed during the pretreatment of the sample.

Chapter 5 details the wetting properties of CNF layers grown on $\mathrm{Si}$ based $\mathrm{Ni}$ thin films. To achieve well-adhesive CNF-layers with a uniform surface coverage and tunable wettability without the necessity of post-synthesis treatments, a series of synthesis parameters is investigated: the pretreatment atmosphere (hydrogen or oxygen; $\left.2 \mathrm{~h}, 500{ }^{\circ} \mathrm{C}\right)$, the use of ethylene $\left(\mathrm{C}_{2} \mathrm{H}_{4}\right)$ or an ethylene/hydrogen $\left(\mathrm{C}_{2} \mathrm{H}_{4} / \mathrm{H}_{2}\right)$ mixture as hydrocarbon source, and the growth time (in the range 5-60 $\mathrm{min}$ ). Ni nanoparticle formation which is required for CNF growth, was only observed when a reductive pretreatment was applied. Fast and uniform CNF-growth is found on 
reduced Ni-based thin films using $\mathrm{C}_{2} \mathrm{H}_{4} / \mathrm{H}_{2}$ at a synthesis temperature of $635{ }^{\circ} \mathrm{C}$. The CNF-layers on Ni are superhydrophobic or highly hydrophobic for all growth times, but their adhesion to the support is poor for growth times $>30 \mathrm{~min}$. In contrast, the adhesion of CNF-layers on Ni/Ta is excellent. Moreover, the wettability of these assynthesized CNF-layers can be controlled by variation of the growth time: from superhydrophobic ( $\leq 10 \mathrm{~min}$ ) to hydrophilic ( $\geq 50 \mathrm{~min}$ ). CNF-layers with such tunable wettability can be easily integrated in flow channels of silicon-based microfluidic systems, thereby offering numerous applications.

The time evolution of the apparent contact angle during evaporation of a water droplet under ambient conditions $\left(\mathrm{T} \sim 23^{\circ} \mathrm{C}, \mathrm{H} \sim 0.3\right)$ on a $\mathrm{CNF}$ sample is studied in chapter 6. On this substrate the contact line remained pinned throughout almost the entire experiment and the evolution of the droplet mass and apparent contact angle with time were carefully recorded and compared with theoretical models. The initial contact angle was as high as $150^{\circ}$, and since it decreased to $0^{\circ}$ during evaporation, a very large range of contact angles could be studied. In our experiments the evaporation turned out to be quasistatic and diffusion-driven, thermal effects play no role. The results quantitatively agree well with a diffusion-based analytical evaporation model. Universal relations for the time evolutions of the droplet mass and contact angle are deduced and the scaling behavior is confirmed by our experimental results.

Droplet impact experiments described in Chapter 7 on superheated silicon and carbon nano-fiber grown silicon surfaces showed that the dynamic Leidenfrost temperature, $T_{L}$ i.e., the transition from the contact boiling to the film boiling regime depends not only on the temperature of the surface and the kinetic energy of the droplet, but also on the size of the structures fabricated on the surface. $T_{L}$ is higher on the CNF surfaces than on the smooth silicon surface. Unlike in microstructured silicon surfaces where increasing pillar height decreased $T_{L}$, in CNF grown silicon surfaces, increasing the fiber length from $3.5 \mu \mathrm{m}$ to $7.5 \mu \mathrm{m}$ causes $T_{L}$ to increase significantly due to the rapid cooling of the nanofibers by the "vapor wind" of the impacting liquid. 


\subsection{General Recommendations and Outlook}

The CNF growth experiments in this thesis show that under the here used experimental conditions the CNF layer thickness increases with increasing growth time and then stabilizes due to catalyst deactivation, bending and entanglement of CNFs. To get a more detailed insight in the growth process in-situ environmental SEM monitoring of the CNF growth process would certainly be an option. One could study this way the nucleation, formation and reaction kinetics of CNF growth, especially in the initial stages. The IR and Raman spectra shown in this thesis demonstrate that these spectroscopic techniques are valuables tools to study the growth of CNF and certainly further analysis of these spectra will pave the way for a more detailed understanding of the different stages in the CNF growth on these $\mathrm{Si}$ substrates. XPS and (in situ) TEM measurements could be used to get additional information on the growth process of CNFs in future research projects.

We showed convincingly (see chapter 5) that wettability can be tuned from nearly hydrophobicity to superhydrophobicity by tuning the growth time. However, an exact relation between the surface morphology and the wetting properties of these surfaces is still lacking, in a large extent due to the heterogeneous nature and complex structure of the CNF layers, reflected in a wide distribution of CNF diameters, bending and entanglement of CNFs for higher growth time. Techniques which gives accurate measurements of CNF surface area, roughness, void volume (porosity) could be helpful here, among which electron tomography would be an obvious choice to start with, because of its capability to resolve $3 \mathrm{D}$ objects.

The possibility to accurately control the growth of CNFs on thin layers in silicon substrates opens the possibility to fabricate and study catalytic reactions in microreactors, using the CNF as a catalyst support. The CNFs will provide a precise control of the concentration of reactants and products at the active side due to their low diffusion resistance, thus minimizing mass transfer limitations, while at the same time the optimized surface to volume ratio of the microreactor is beneficial to reduce heat transfer limitations.

A generally accepted notion is that wetting (hydrophilic) surfaces obey the noslip boundary condition whereas clean non-wetting (hydrophobic) surfaces exhibits 
apparent slip, of the order of microns $(\sim 1000$ times longer than the molecular size or mean free path) often to a surprisingly large extent. ${ }^{[1]}$ Our initial experiments showed that these layers could be successfully grown directly in micro-reactors. The possibility to tune the wettability of well-adhesive, as-synthesized CNF-layers without post-synthesis treatments- enables the systematic variation of the wettability in flow channels of silicon-based microfluidic systems by a CNF coating, thus enabling a systematic study of the presence (or absence) of slippage in CNF coated microreactors by micro-particle image velocimetry. This information would, in addition, be relevant for catalytic reaction studies in microreactors. For example, hydrophilic CNF-layers can be used as catalyst support in microreactors and superhydrophobic CNFs as coatings for drag reduction (super-lubrification).

An attractive proposition for further research in line of the evaporation studies conducted in chapter 6 , is the effect of surfactants and salt being present in the evaporating water droplets, as well as the evaporation of various other liquids, like ethanol, FC-72 and olive oil. Beside droplets of one liquid, one could also think of evaporation or wetting studies of mixed liquids.

For the study of the dynamic Leidenfrost temperature, presented in chapter 7, an accurate value of surface area, density and void volume of the CNF surface would potentially lead to a better understanding of the observed phenomena. Unfortunately, BET surface area measurements were not reliable due to the low amount of carbon produced. One option is to grow CNFs on substrates fabricated with bigger dimension in order to reach a reasonable amount of CNFs which could give proper BET surface area and void volume measurements. In addition, a careful experimental determination of the thermal conductivity and thermal diffusivity of the CNFs used in this study is lacking.

Impacting droplets on superheated CNF surfaces at various Weber and Reynolds number may result in a wealth of phenomena, including jet formation, micro-droplet formation, splashing. ${ }^{[2]}$ Further experimental investigation of the dynamics after droplet impact, will certainly result in new and exciting fundamental discoveries and are highly relevant for industrial applications like inkjet printing, spray cooling and spray painting. 


\subsection{References}

[01] T. M. Squires, S. R. Quake, Rev. of Mod. Phys., 2005, 77, 977.

[02] T. Tran, H. J. J. Staat, A. Susarrey-Arce, T. C. Foertsch, A. van Houselt, J. G. E. Gardeniers, A. Prosperetti, D. Lohse, and C. Sun, Soft Matter, 2013, 9, 3272. 



\section{SAMENVATTING IN HET NEDERLANDS}

(SUMMARY IN THE DUTCH)

De bevochtigingseigenschappen van oppervlakken worden bepaald door de oppervlakte vrije energie en de ruwheid. In dit proefschift worden de groei en bevochtigingseigenschappen van koolstof nanodraden bestudeerd.

Hoofdstuk 1 vormt in inleiding op koolstof nanodraden en bevochtiging, de belangrijke onderwerpen in dit proefschrift. De bestudering van koolstof nanodraden wordt in een historisch perspectief geplaatst en de groeiende interesse in (super) waterafstotende oppervlakken wordt geïntroduceerd.

In hoofdstuk 2 wordt het verdampen van een water druppel op een silicum oppervlak voorzien van een textuur op micrometer schaal en een FOTS coating bestudeerd. Een door het verdampingsproces geïnduceerde Cassie-Baxter naar Wenzel overgang voor de druppel, zoals eerder gevonden is voor super waterafstotende oppervlakken, wordt op deze oppervlakken, die naast waterafstotend ook olieafstotend blijken te zijn, niet of veel later geobserveerd. Een verklaring op basis van een vergelijking van de oppervlakte vrije energie van de verdampende druppels, wat voor micropilaren met een scherpe rand en een glad oppervlak een correcte beschrijving van de Cassie naar Wenzel overgang oplevert, kan deze overgang voor de water- en olieafstotende oppervlakken niet verklaren. Het verschuiven of uitblijven van deze overgang wordt in dit geval toegeschreven aan een extra energie barrier ten gevolge van de toegevoegde grotere kromtestraal van de rand en grotere ruwheid van de oppervlakken van de micropilaren.

Hoofdstuk 3 beschrijft de invloed van de van de voorbehandeling van samples die voorafgaat aan het groeiproces van de koolstof nanodraden op de bevochtigingseigenschappen van de dunne nikkel (Ni) laag. Ni lagen met variërende dikte en hechtingslagen - $10 \mathrm{~nm} \mathrm{Ni,} 25 \mathrm{~nm}$ Ni en $25 \mathrm{~nm}$ Ni op $10 \mathrm{~nm}$ Tantaal (Ta) als hechtlaag - zijn gegroeid op een geoxideerd silicium substraat als dragermateriaal voor een te groeien koolstof nanodraden. Deze lagen werden blootgesteld aan 
verschillende voorbehandelingen (namelijk verschillende temperaturen en tijdsduur) in vacuum, lucht, stikstof of waterstof omgeving. Voor een voorbehandeling van één uur op $650{ }^{\circ} \mathrm{C}$ werd alleen in de aanwezigheid van waterstof gas de vorming van separate Ni nanodeeltjes (aangetoond door energie dispersieve röntgen analyse) geobserveerd. Voor de $25 \mathrm{~nm}$ Ni op $10 \mathrm{~nm}$ Ta resulteerde deze voorbehandeling in een scherp rond $56 \mathrm{~nm}$ gepiekte deeltjesgrootte verdeling.

Voor de Ni dunne lagen die voorbehandeld zijn in vacuum of stikstof resulteerde blootstelling aan condities die idealiter zouden kunnen leiden tot groei van koolstofnanodraden, in dit geval tot de vorming van amorfe koolstoflagen op niet volledig in nanodeeltjes gefragmenteerde dunne Ni lagen. Afhankelijk van de koolstofnanodraden groei tijd en de aanwezigheid van een Ta laag zijn de koolachtige lagen continu of poreus, zonder de vorming van koolstofnanodraden. In lucht voorbehandelde samples resulteren voor groeitijden vanaf een minuut in koolstofnanodraden tapijten, die echter niet stevig verankerd zijn aan het substraat. Er wordt geconcludeerd dat de voorbehandeling van de Ni dunne lagen in vacuum, stikstof of lucht niet resulteert goed gebonden, homogene koolstofnanodraden lagen. Voor samples met een Ta hechtlaag worden homogeen gedistribueerde, goed verankerde, semikristallijne kooldraden gegroeid binnen een groetijd van 3 minuten. De diameter van deze draden varieert tussen 15 en $40 \mathrm{~nm}$, wat goed overeenkomt met de deeltjesgrootte verdeling na de reducerende voorbehandeling. Afwezigheid van de Ta hechtlaag resulteert in de vorming van nanodeeltjes van enkele honderden nanometers in diameter, waarop een amorfe koollaag wordt gevormd, maar geen koolstof nanodraden worden geobserveerd. Nanodeeltjes met een diameter kleiner dan $80 \mathrm{~nm}$ blijken een vereiste te zijn voor koolstofnanodradengroei. Voor de condities die in deze studie zijn gehanteerd, was dit alleen het geval voor een gereduceerde film van $25 \mathrm{~nm}$ Ni op een $10 \mathrm{~nm}$ Ta hechtlaag.

In een poging om meer inzicht te genereren in het groeiproces and de aard van de gesynthetiseerde koolstofnanodraden lagen zijn in hoofdstuk 4 de monsters met koolstofnanodraden met verschillende groeitijden (varierend van enkele minute tot een uur) gekarakteriseerd door Raman en Infrarood spectroscopie en hoge resolutie electronen en He ionen microscopie. Tijdens de groei van de koolstofnanodraden blijkt zich op de samples zonder Ta hechtlaag een dunne bijna vrij staande 
koolstoflaag te vormen. Deze laag heeft grafiet karakteristieken en ontwikkeld zich waarschijnlijk door de vorming van silicium carbide laag, die gedurende de voorbehandeling van het monster ontstaat.

In hoofdstuk 5 worden de bevochtigingeigenschappen van de kooldraden lagen gegroeid op de Ni dunne lagen bestudeerd. De voorbehandelings atmosfeer (blootstelling aan waterstof of zuurstuf gedurende 2 uur op $500{ }^{\circ} \mathrm{C}$ ), het gebruik van een ethyleen of ethyleen/waterstof mengels als koolstof bron en de groeitijd ( $5-60$ min) zijn systematisch gevarieerd. De vorming in gefragmenteerde Ni nanodeeltjes, die noodzakelijk zijn voor de kooldradengroei, werd alleen gezien bij een reducerende voorbehandeling. Voor deze gereduceerde samples werd bij een groeitemperatuur van $635{ }^{\circ} \mathrm{C}$ de vorming van een uniforme koolstofdradenlaag geobserveerd. De koolstofnanodraden die gegroeid werden op $\mathrm{Ni} / \mathrm{SiO}_{2}$ lagen vertoonden waterafstotend gedrag voor alle groeitijden, maar echter met een slechte binding aan het dragermateriaal voor groeitijden lager dan 30 minuten. Kooldraden gegroeid om Ni lagen op een Ta hechtlaag bleken wel een goede adhesie aan het dragermateriaal te vertonen en op deze lagen kan bovendien door het variëren van de groeitijd de bevochtiging gecontroleerd worden van zeer waterafstotend $(\leq 10 \mathrm{~min})$ tot waterlievend ( $\geq 50 \mathrm{~min}$ ). Deze koolstofnanodraden kunnen gemakkelijk toegepast worden in vloeistof kanalen van Si gebaseerde microfluidische systemen.

In hoofdstuk 6 wordt de contacthoek van een verdampende waterdruppel $\left(\mathrm{T} \sim 23^{\circ} \mathrm{C}, \mathrm{H} \sim 0.3\right)$ op een koolstofnanodradensample gevolgd in de tijd. Op dit substraat blijkt de contactlijn gedurende bijna het gehele experiment gepind te zijn en de veranderingen in de massa van de druppel en de contacthoek werden zorgvuldig vergeleken met theoretische modellen. De initiële contacthoek tijdens de verdamping was $150^{\circ}$ and deze reduceerde gedurende het experiment gelijdelijk tot uiteindelijk $0^{\circ}$. De verdamping van waterdruppels op deze oppervlakken blijkt diffusie-gedreven te zijn, zonder waarneembare thermische effecten. De experimentele resultaten komen goed overeen met een diffusie gebaseerd analytisch verdampingsmodel. Op basis van dit model zijn universele relaties voor het tijdsverloop van de massa en contacthoek van de druppel afgeleid. 
De experimenten beschreven in hoofdstuk 7, waarbij druppels met een bekende snelheid op een verhit $\mathrm{Si}$ and koolstofnanodraden/Si substraat vallen tonen aan dat de dynamische Leidenfrost temperatuur $T_{L}$, de overgangstemperatuur tussen direct contact met de kokende druppel naar de aanwezigheid van een dunne waterdamplaag, niet alleen afhangt van de kinetische energie en de temperatuur, maar ook van de grootte van de structuren die op het oppervlak aanwezig zijn. De dynamische Leidenfrost temperatuur $T_{L}$ is hoger voor de kooldraden oppervlakken dan voor de vlakke siliciumoppervlakken. In tegenstelling tot de observaties bij microgestructureerde silicium oppervlakken, waarbij een hogere pilaarlengte leidt tot een lagere $T_{L}$. wordt voor de koolstofnanodraden oppervlakken bij een toename van de kooldradenlengte van $3.5 \mu \mathrm{m}$ naar $7.5 \mu \mathrm{m}$ een significante toename in $T_{L}$ geobserveerd, als gevolg van de snelle afkoeling van de nanodraden door de koele dampstroom die aan de vallende druppel voorafgaat.

$* * * * * * * * * * * *$ 


\section{SCIENTIFIC CONTRIBUTIONS}

\section{JOURNAL Publications:}

- $\quad$ H. Nair and H.J.J. Staat, T. Tran, A. van Houselt, A. Prosperetti, D. Lohse and C. Sun, "The Leidenfrost temperature increase for impacting droplets on carbon-nanofiber surfaces." Soft Matter, 2014, 10, 2102.

- H. Nair, R.M. Tiggelaar, D.B. Thakur, J.G.E. Gardeniers, A. van Houselt, L. Lefferts, "Evidence of wettability variation on carbon nanofiber layers grown on oxidized silicon substrates." Chemical Engineering Journal, 2013, 227, 56.

- H. Nair, G.R. Cruellas, R.M. Tiggelaar, L. Lefferts, A. van Houselt, “ A Raman and infrared study of carbon nanofiber growth.” (in preparation)

- R.M. Tiggelaar, D.B. Thakur, H. Nair, L. Lefferts, K. Seshan, J.G.E. Gardeniers , "Influence of thin film nickel pretreatment on catalytic thermal chemical vapor deposition of carbon nanofibers." Thin Solid Films, 2013, 534, 341 .

- A. Susarrey-Arce, A.G. Marin, H. Nair, J.G.E. Gardeniers, L. Lefferts, D. Lohse and A. van Houselt, "Absence of an evaporation-driven wetting transition on omniphobic surfaces." Soft Matter, 2012, 8, 9765.

- H. Gelderblom, A.G. Marin, H. Nair, A. van Houselt, L. Lefferts, J. H. Snoeijer and D. Lohse, "How water droplets evaporate on superhydrophobic CNF substrate." Physical Review E., 2011, 83, 026306.

\section{ORAL Presentations and Contributions:}

- $\quad$ H. Nair, R.M. Tiggelaar, D.B. Thakur, J.G.E. Gardeniers, A. van Houselt, L. Lefferts, "Superhydrophobic CNFs for slippage studies in microreactors", International conference on microreaction technology, February 20- 22, 2012, Lyon, France.

- H. Nair, R.M. Tiggelaar, A. van Houselt, L. Lefferts, "Wettability control on carbon nanofiber surfaces: Optimization of growth parameters", Carbon-based nanomaterials and devices, October 16-21, 2011, Suzhou, China.

- H. Nair, R.M. Tiggelaar, A. van Houselt, L. Lefferts, "Control of wettability on carbon nanofiber surfaces: from hydrophilicity to superhydrophobicity", Diamond 2011, September 4- 8, 2011, Bavaria, Germany.

- H. Nair, H. Gelderblom, A.G. Marin, A. van Houselt, L. Lefferts, J. H. Snoeijer, D. Lohse, "Droplet evaporation on superhydrophobic carbon nano 
fiber surfaces", $12^{\text {th }}$ Netherlands Catalysis and Chemistry Conference, March 1-3, 2011, Noordwijherkout, The Netherlands.

- A. Agiral, H. Nair, K. Seshan, L. Lefferts, J.G.E. Gardeniers, "Carbon nanofiber deposition in microreactors by atmospheric pressure microplasma", $8^{\text {th }}$ Netherlands Process Technology Symposium, October 27-29, 2008, Veldhoven, Netherlands.

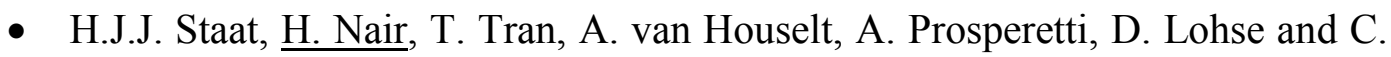
Sun, "The Leidenfrost temperature increase for impacting droplets on carbonnanofiber surfaces", 67 ${ }^{\text {th }}$ Annual Meeting of the APS-DFD, November 23-25, 2014, San Francisco, USA.

\section{POSTER Presentations and Contributions:}

- H. Nair, A. van Houselt, D. Lohse and L. Lefferts, "Catalytic growth of superhydrophobic surfaces", $11^{\text {th }}$ Netherlands Catalysis and Chemistry Conference, March 2010, Noordwijherkout, The Netherlands.

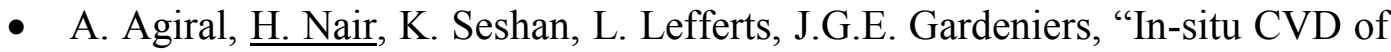
CNFs in a microreactor", III International Symposium on Carbon for Catalysis, November 9-12, 2008, Berlin, Germany,.

- A. Agiral, H. Nair, K. Seshan, L. Lefferts, J.,G.E. Gardeniers, "Carbon nanofiber deposition in microreactors by atmospheric pressure microplasma", $7^{\text {th }}$ Netherlands Process Technology Symposium, October 27-29, 2008, Veldhoven, The Netherlands.

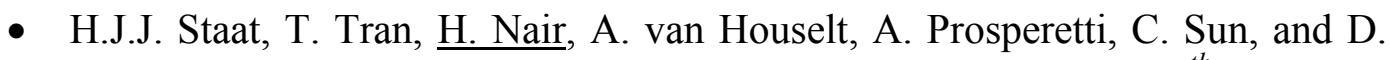
Lohse, "Exploding droplet", Gallery of Fluids Motion Poster, $66^{\text {th }}$ American Physical Society-Division of Fluid Dynamics meeting, November 24-26, Pittsburgh, Philadelphia, USA.

- H.J.J. Staat, T. Tran, H. Nair, A. Susarrey-arce, A. van Houselt, J.G.E. Gardeniers, A. Prosperetti, C. Sun, and D. Lohse, "Tuning the dynamic Leidenfrost temperature", Micro-Nano conference 2013, 11-12 December 2013, De ReeHorst, Ede, The Netherlands.

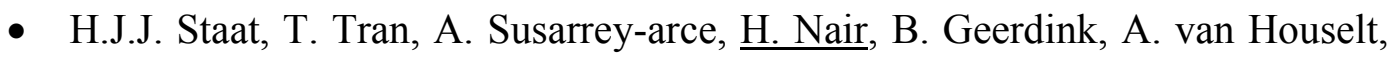
J.G.E. Gardeniers, A. Prosperetti, C. Sun, and D. Lohse, "Droplet impact on superheated surfaces”, Physics@FOM, Veldhoven, 21-22 January 2014, The Netherlands.

\section{Student Supervision:}

- B.Sc Project: Sander Clahsen

- MS project: Gonzalo Rios Cruellas 


\section{ACKNOWLEDGEMENTS}

The last few years of my life at the University of Twente in Netherlands have been very memorable and I thank the university and everyone who, directly or indirectly have been a great source of support for me during my stay and research there. It's my pleasure to thank my promoters Prof. Leon Lefferts and Prof. Detlef Lohse for offering me this challenging and wonderful opportunity to learn science in a multidisciplinary research area combining catalysis, fluid physics and Nanotechnology.

I extend my heartfelt gratitude to Prof. Leon for the supervision and support he offered throughout my research. The scientific discussions, encouragements, constructive criticisms and feedbacks during the progress meetings were crucial and motivated me to view limitations as challenges. Your support and confidence in letting me do the research in CPM motivated me a lot. It was great to spend time with Ineke and your family during CPM annual gatherings.

I wholeheartedly thank Prof. Detlef for the freedom offered in research and for the priceless support and encouragement, quick responses, and guidance without which this thesis would not have materialized. You have been a source of inspiration and your extraordinary scientific and leadership qualities helped my research and made almost everything possible in PoF, making it one of the dream locations for students who are passionate about research.

I express my heartfelt gratitude to my daily supervisor, Dr. Arie van Houselt for his guidance and trust, and the freedom and flexibility offered in research and collaborations throughout my research period. There is a lot to learn from you about scientific writing, presentation skills and personality development. The discussions and skype meetings, especially during my stay abroad, helped me a lot and I am indebted to you for the patience, understanding, friendship, advices, constructive criticisms, care and moral support you offered in all the times. Your encouragement was a great stress-reliever and without your sincere support this thesis would not have 
materialized. Thanks a lot for helping me with the Dutch summary, thesis proof-print checks and for sharing the beautiful photographs in chapter 1. It was nice to meet Agatha and Philip, Jarno, Corne and Agathe at your home and over skype.

I thank Prof. Han Gardeniers for his support and invaluable guidance in the dewetting of metal film project. I wholeheartedly thank Dr. Roald M. Tiggelaar for his kind support and guidance throughout my research and for the enormous contribution to my thesis and personal development. Your quick responses and feedbacks, constructive criticisms, expertise in metal thin films and dewetting and micro-device fabrication projects in cleanroom helped me a lot to accomplish my research.

I thank Dr. Chao Sun for his encouragements, discussions, kind support and supervision in PoF droplet impact project, which meant a lot to me. I thank Dr. Tuan Tran for his guidance and expertise, and Erik-Jan for his support in experiments and matlab programs. It was great to work with your team, without whose timely support and enormous contribution this thesis would be incomplete.

I express my heartfelt gratitude to Prof. A. Prosperetti for his quick responses, invaluable support and guidance offered in the droplet impact project. I also thank Prof. Devaraj, Prof. Jacco, especially for his enormous theoretical contributions to chapter 6, Prof. Michel, Prof. Leen, Prof. Frieder, Dr. Srirang for being very kind, friendly and supportive.

I thank Prof. Daniel Bonn, Prof. Harry Bitter and all my committee members for being a part of my graduation committee and in particular, Prof. Hans Hilgenkamp for his quick responses, timely help and encouragement in the last stage of my research.

I am extremely grateful to Prof. K. Seshan and Jayanti for their moral support, constructive criticisms, care and encouragement and the homely atmosphere I enjoyed in your house. I thank Dr. Barbara L. Mojet for her love, understanding and kind support offered to my research.

This thesis is the realization of fruitful co-operation and I gratefully thank everyone who contributed to this thesis. My special thanks to the CNF team, especially Dr. Jitendra, Dr. Digvijay and Dr. Sergio for sharing the basic knowledge viii 
about CNFs and catalytic synthesis and for the stimulating discussions we had, which helped me a lot in my research. I thank Dr. Arturo for his microstructures, Dr. Alvaro for his expertise, guidance and experimental support, and Prof. Jacco and Dr. Hanneke for the theoretical contribution to the droplet evaporation project, Gonzalo and Sander for their great contribution to the spectroscopy project, Rao for the spectroscopy experiments and discussions, Dr. James Seddon for his suggestions in chapter 2, Dr. Amy for the collaborations on $\mu$-PIV, Dr. Anil for the micro-plasma CNF growth experiments, SFI group for the use of their contact angle meter, Mark Smithers and Gerard for the SEM images and Gregor for the HIM images.

I am extremely grateful to Bert Geerdink for his invaluable support, guidance, fatherly care and moral support throughout my research and for his magical touch on computers and experimental set-ups which made life easier. Thanks a lot for designing a beautiful thesis cover, lay-out and chapter 8-abstract inset image and for the quick feedbacks on thesis proof-print. It was great meeting your wife Elisabeth and thanks for being very kind, understanding and friendly.

I am grateful to our secretaries Lidi, Sabina, especially Lianne, Joanita and Maaike for helping me at crucial times including the visa related issues, arrangement of meetings and defense. I also thank Astrid, Marieke and Santra for their help in various organizational matters. I thank technicians Tom, Karin, Louise, Stefen, Samanta, Peters, Hans and especially Ruben, Martin and Gert-Wim for the technical support and Bas for his invaluable quick help in fixing computer and software-related issues, which saved time and made life easier.

It is my pleasure to have my friends Chau and Marijana as paranymphs and I thank them especially for their kind support and help in this last stage of research and defense. Friendships are a blessing and I thank all my colleagues and friends, especially Jitendra, Roald, Rao, Vivek, Dennis-Astrid, Arturo, Gonzalo, Chris, Sergio, Vijay, Shilpa, Sander, Igor, Dejan, Son, Siddhartha, Tuan, Erik-Jan, Yoshi, Alvaro, Rajaram, James, Daniel, Julian, SanderH, Bram, Ivo, Ceyda, Samuel, Laura, Hanneke, Alhadidi, Akram, Berta, Gacia, Inga, Liza, Christiano, Davide, Joline, Mazoud, Songbo, Kamilla, Roger, Yingnan, Khalid, Bart, Kaisa, Regina, Federico, SanderW, 
Sylvian, Olexander, Tak, Henri, Wim, Gor, Roeland, Alisia for their warm support and for the great fun, discussions and relaxing times we enjoyed in the office and lab.

No words of thanks would be enough to express my gratitude towards Kamala aunty-Bernie uncle, Meegha-Antony, Sandhya-Rao, Jalaja-Sandeep, Dileep, Jithin, Lavanya-Jitendra, Indu-Babu, Marijana, Ragav, Anand, Ranjini-Vijay and Yogita for their warm support and care throughout my stay and the homely atmosphere I enjoyed in Netherlands. I also thank my friends Shahina-Musthafa, Sumy, Banu, SowjanyaSreekumar, Rajesh, Jincy, Rani, Simi-Chinthan, Aabha-Digvijay, Subhashini-Anand, Selina-Amtuladhar, Bindu-Sekhar, Denny-Abraham, Vishnu, Vinay, Pramod, Vidhu, Mercy-Shaji, Vishaka-Pramod, Varsha-Giri, Priyanki, Neeru, Sharmila-Rajaram, Srivatsa, ISA, and Aadhaar team for making my stay memorable in the Netherlands.

I thank my teachers in Bappuji School, Vimalagiri School, Alphonsa college, Cyber Prism, Mahatma Gandhi University, IIT Madras and Raman Research Institute Bangalore and project guides, Dr. Vincent Mathew, Prof. C. Vijayan, Prof. V. A. Raghunathan for their everlasting support, care and guidance without which I would be nothing.

I take this opportunity to thank all relatives and friends of my family for the everlasting support, love and care shown to me. I also thank sister-in-law Anushree, parents Prema Ullattil and Dr. M. Vijayanunni and relatives for their moral support, prayers and understanding. No words of thanks would be enough to express my sincere gratitude and love towards my grandparents, aunts and uncles, cousins and above all my sister Dr. Harsha Nair, brother-in-law Vivek P.R., husband V. Abhijit and parents Shyla Nair and K. Vijayakumaran Nair for their unending love, prayers, guidance, constructive criticism, understanding, care and everlasting support throughout. Staying away from you all was the greatest challenge I had to face and thanks for the support in the most needy times and I owe this thesis to you. You are my strength and I love you so much. Above all, I thank the Almighty for giving me a loving family, many wonderful opportunities in life and for all the blessings showered upon me. 


\section{ABOUT THE AUTHOR}

Hrudya Nair was born in Koothattukulam, Ernakulam district, Kerala in India. She completed B.Sc. in Physics from Alphonsa College, Pala and M.Sc. in Physics from School of Pure and Applied Physics, Mahatma Gandhi University Campus, Kottayam, Kerala. She worked on theoretical survey of metamaterials and synthesis and optical characterization of semiconductor nanoparticles as part of M.Sc. project under Prof. C. Vijayan, Department of Physics, Indian Institute of Technology, Madras. She also worked on counterion-induced structural transformation of DNAsurfactant complexes in Raman Research Institute (RRI), Bangalore under Prof. V. A. Raghunathan, as part of a visiting student fellowship from RRI and Summer Research Fellowship from Indian Academy of Sciences, Indian National Science Academy and National Academy of Sciences. Thereafter she joined university of Twente, Netherlands for her $\mathrm{PhD}$ and this thesis is the outcome of her research.

\section{ABOUT MY THESIS COVER}

Nature is very inspiring and always aroused the curiosity of scientists. The scenery used in the background of this thesis is taken in Munnar, Kerala. The wetting properties displaced by the leaves and flowers in nature and their interesting internal structure inspired scientists to learn the fundamentals and to apply it to develop surfaces with varied wettability for different applications in day-to-day life. The dense forest in the cover symbolizes the carbon nanofiber (CNF) forests which are the basic substrates used in this research. The beautiful rising sun in the front cover and the droplet hat and umbrella pictures in the back cover are the various shapes obtained by impacting a millimetric water droplet on a heated CNF surface. The impacting sequence of an FC-72 drop on a heated CNF surface, studied in chapter 7, is used near the peak of the hill in the invitation letter. The cover also symbolizes the light shining in life (sunshine) after a period of darkness (sunset). 


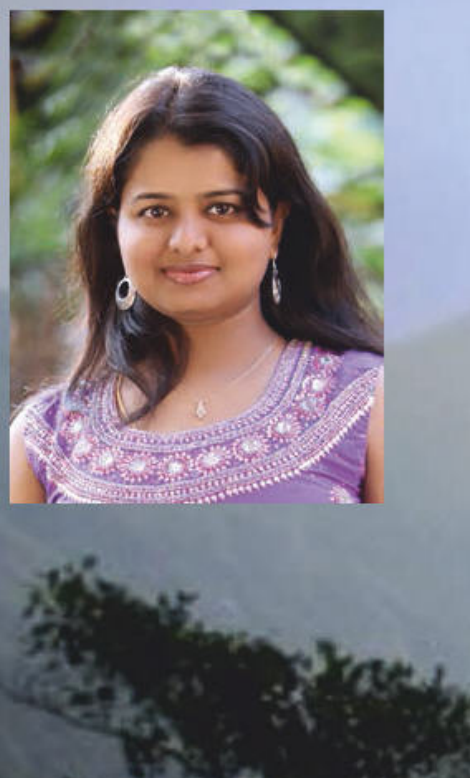

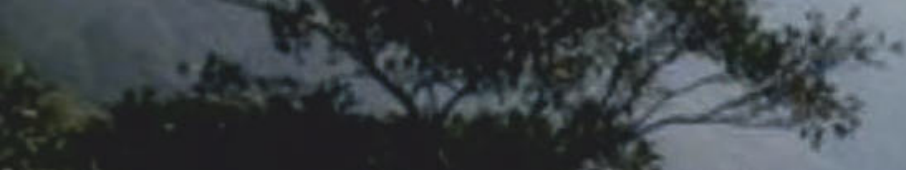

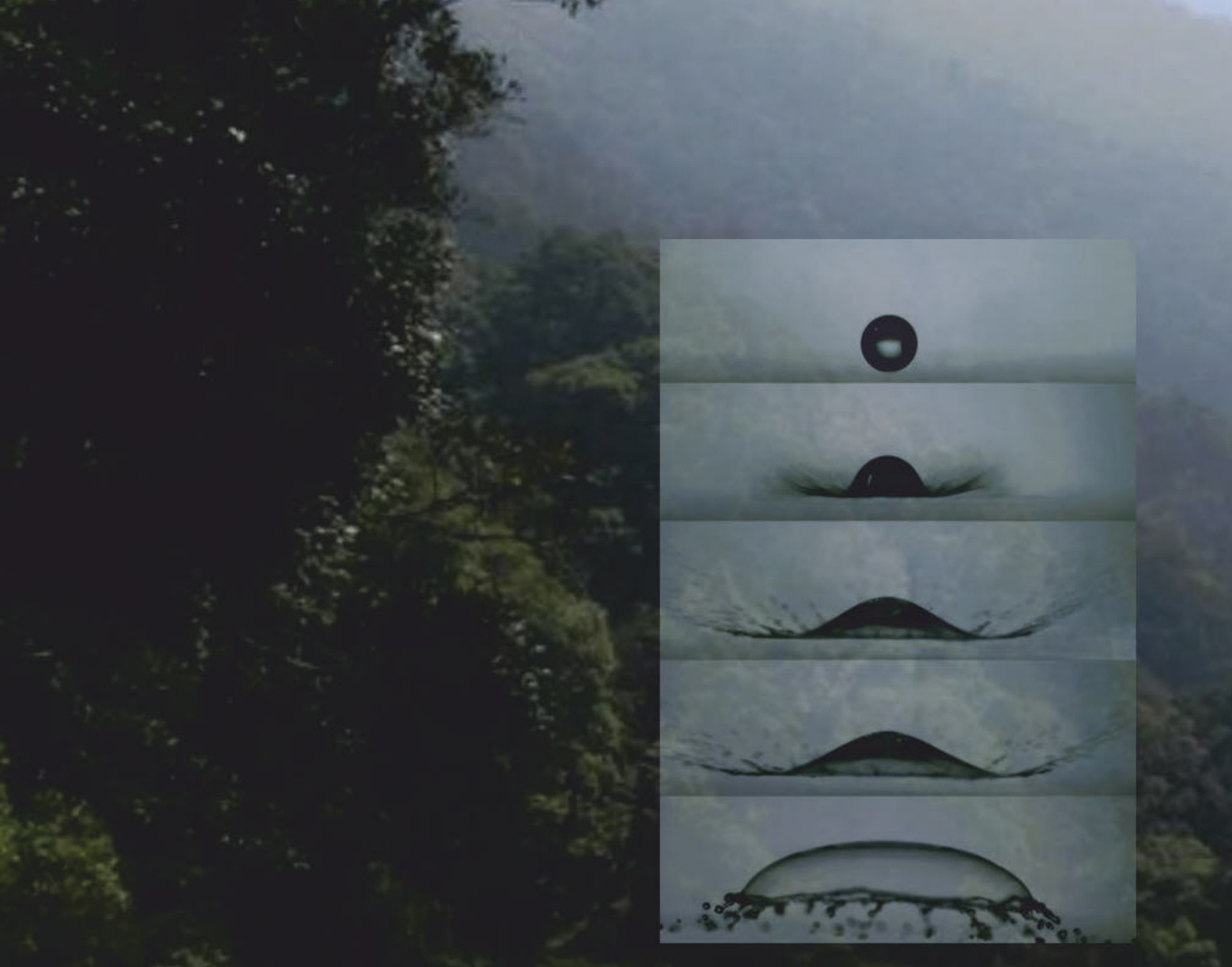

